$\gamma$

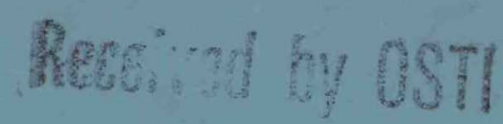

IJUN 041990

NUREG/CR-5436

SAIC-90/1130

Vol. 2

\title{
The Development and Evaluation of Programmatic Performance Indicators Associated with Maintenance at Nuclear Power Plants
}

\section{Appendices}

Prepared by J. Wreathall, J. Fragola, P. Appignani,

G. Burlile, Y. Shen

Science Applications International Corporation

Prepared for

U.S. Nuclear Regulatory Commission

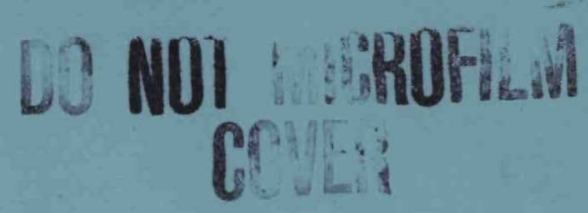




\section{AVAILABILITY NOTICE}

Availability of Reference Materials Cited in NRC Publications

Most documents cited in NRC publications will be avallable from one of the following sources:

1. The NRC Public Document Room, 2120 L Street, NW, Lower Level, Washington, DC 20555

2. The Superintendent of Documents, U.S. Government Printing Office, P.O. Box 37082 , Washington, DC 20013-7082

3. The National Technical Information Service, Springfield, VA 22161

Although the listing that follows represents the majority of documents cited in NRC publications, it is not intended to be exhaustive.

Referenced documents avallable for inspection and copying for a fee from the NRC Public Document Room Include NRC correspondence and internal NRC memoranda; NRC Office of Inspection and Enforcement bulletins, circulars, information notices, Inspection and Investigation notices; Licensee Event Reports; vendor reports and correspondence; Commission papers; and applicant and licensee documents and correspondence.

The following documents in the NUREG serles are avallable for purchase from the GPO Sales Program: formal NRC staff and contractor reports, NRC-sponsored conference proceedings, and NRC booklets and brochures. Also avallable are Regulatory Guides, NRC regulations in the Code of Federal Regulations, and Nuclear Regulatory Commission Issuances.

Documents avallable from the National Technical Information Service include NUREG series reports and technical reports prepared by other federal agencies and reports prepared by the Atomic Energy Commisslon, forerunner agency to the Nuclear Regulatory Commission.

Documents avallable from public and special technical libraries include all open literature items, such as books, Journal and periodical articles, and transactions. Federal Register notices, federal and state legislation, and congressional reports can usually be obtained from these librarles.

Documents such as theses, dissertations, foreign reports and translations, and non-NRC conference proceedings are avallable for purchase from the organization sponsoring the publication cited.

Single coples of NRC draft reports are avallable free, to the extent of supply, upon written request to the Office of Information Resources Management, Distribution Section, U.S. Nuclear Regulatory Commission, Washington. DC 20555.

Coples of Industry codes and standards used in a substantive manner in the NRC regulatory process are maintained at the NRC Llbrary, 7920 Norfolk Avenue, Bethesda, Maryland, and are avallable there for reference use by the public. Codes and standards are usually copyrighted and may be purchased from the originating organization or, If they are American National Standards, from the American National Standards Institute, 1430 Broadway, New York, NY 10018.

\section{DISCLAIMER NOTICE}

This report was prepared as an account of work sponsored by an agency of the United States Government. Neither the United States Government nor any agency thereof, or any of their employees, makes any warranty, expresed or implied, or assumes any legal liability of responsibility for any third party's use, or the results of such use, of any information, apparatus, product or process disclosed in this report, or represents that its use by such third party would not infringe privately owned rights. 


\section{The Development and Evaluation} of Programmatic Performance Indicators Associated with Maintenance at Nuclear Power Plants

Appendices

Manuscript Completed: August 1989

Date Published: May 1990

Prepared by

J. Wreathall, J. Fragola, P. Appignani,

G. Burlile, Y. Shen

Science Applications International Corporation 2929 Kenny Road

Columbus, $\mathrm{OH} 43221$

Prepared for

Office of Nuclear Regulatory Research

U.S. Nuclear Regulatory Commission

Washington, DC 20555

NRC FIN L1183 


\section{DISCLAIMER}

This report was prepared as an account of work sponsored by an agency of the United States Government. Neither the United States Government nor any agency Thereof, nor any of their employees, makes any warranty, express or implied, or assumes any legal liability or responsibility for the accuracy, completeness, or usefulness of any information, apparatus, product, or process disclosed, or represents that its use would not infringe privately owned rights. Reference herein to any specific commercial product, process, or service by trade name, trademark, manufacturer, or otherwise does not necessarily constitute or imply its endorsement, recommendation, or favoring by the United States Government or any agency thereof. The views and opinions of authors expressed herein do not necessarily state or reflect those of the United States Government or any agency thereof. 


\section{DISCLAIMER}

Portions of this document may be illegible in electronic image products. Images are produced from the best available original document. 


\begin{abstract}
This report summarizes the development and evaluation of programmatic performance indicators of maintenance. These indicators were selected by: (1) creating a formal framework of plant processes; (2) identifying features of plant behavior considered important to safety; (3) evaluating existing indicators against these features; and (4) performing statistical analyses for the selected indicators. The report recommends additional testing.
\end{abstract}


' 


\section{Table of Contents}

Appendix A, Synopsis of Process Model

Appendix B, Detailed Results of Statistical Analysis B-1

Attachment 1, Signal Processing Analysis of Att-1-1 Daily Power Loss Indicator 
Appendix A, Synopsis of Process Model 


\section{Appendix A, Synopsis of Process Model}

\section{A.1 Functional Units}

The following are summary descriptions of the principal functional units within a typical nuclear power plant that are included in Figure 3.2.

\section{Operations}

The operations unit comprises the power plant staff who are responsible for ensuring that the plant is as close to the targeted economic power-production state as safety and equipment constraints permit. This unit includes the control-room crew (shift supervisors, senior reactor operators, and reactor operators) and the plant area operators. This unit is almost always a separate, self-contained administrative unit within the plant.

\section{Maintenance}

The maintenance unit comprises the staff responsible for assuring the availability and operability of plant systems to ensure that targeted levels of economic power production are achieved with the required level of safety. Maintenance tasks include diagnosing and repairing component failures, performing preventive maintenance, and performing surveillance testing. In a sense, the maintenance unit's responsibility is to preserve and enhance the "assets" of the plant--the physical equipment. This unit is again usually a separate administrative unit within the plant; in a broad sense, some of the other functional units may be considered part of maintenance, including, for example, performance analysis.

\section{Training}

The training unit is a service unit, either part of the corporate (headquarters) structure or part of the site organization, that is responsible for transferring knowledge and skills to the staff in the other areas, including operators and maintenance personnel. Training includes classroom instruction, practice with mock-ups, and practice with simulators (for operations staff).

\section{Quality Assurance}

Quality assurance (QA) is a unit in the utility whose function is to provide an independent appraisal of safety-related work by maintenance, operations, health physics, and other groups affecting safety. QA performs this function in two basically similar ways; it typically reviews and checks work in progress by maintenance personnel at identified check points, and it monitors and audits performance by other groups, including operations, health physics, etc., on a periodic basis. QA is required to provide reports independently to a senior utility manager, but typically provides liaison with site management in order to provide opportunities for direct problem resolution. 


\section{Performance Analysis}

The performance analysis group is responsible for monitoring, tracking and recordkeeping of plant performance (including station generating performance), and equipment performance. This group may be located administratively in one of several groups, including operations or plant engineering, or may be a separate group within the plant.

\section{Plant Engineering (On-site)}

This group is responsible for performing technical evaluations of systems' performance, such as heat-loss calculations, fuel burnup, and equipment reliability tracking. Root-cause analyses of unusual or repeated equipment failures will be performed here. This group is also responsible for identifying plant modifications needed to eliminate problem areas or perform moderate upgrades to systems in conjunction with corporate engineering staff. Staff assigned as Shift Technical Advisors and other emergency response staff will usually be part of this group. This group will be an independent unit on site.

\section{Purchasing/Construction (On-site)}

The purchasing/construction group perform the tasks of ordering and purchasing new equipment for plant modifications, and for carrying out their installation. For major modifications, this group will work in conjunction with corporate staff and vendor installation teams. This group may be part of other administrative departments or a separate department. At some plants, this group may be split into purchasing and construction, each of which is part of another department.

\section{Site Management}

The site management is responsible to the corporate management for the safe, economic operation of the plant. It accomplishes this by (in a formal sense) the allocation of resources, the setting of department goals and the evaluation of performance; management also largely functions through continuous informal and semiformal discussions with the other groups, and the coordination and sharing of information. This group (at the top level) is a separate unit (station manager, etc.); at the lower levels, department managers are both part of the site management group and members of the other various groups.

\section{Other Plant Functions}

There are several groups that are involved in plant operations, including health physics, chemistry, security, and so on, that are grouped together for the purposes of this study. In addition, there are numerous administrative support groups that are located at the site. For this study, they are treated as an additional functional area for completeness but are not believed to have a major interface with training or maintenance in terms of their safety roles. 


\section{A.2. Primary Function Interfaces}

activities:

The following are the primary interfaces between the most important functional

\section{Maintenance}

(a) Site Management: Site management provides the maintenance unit with goals and resources. In some plants the goals may be formalized such as the reduction of forced outages due to maintenance error to less than " $\mathrm{x}$ per fuel cycle" or some similar set of goals. In some plants, explicit maintenance goals may not be set. The resources are primarily manpower and dollar budgets. Such resources may identify overtime requirements explicitly or implicitly.

(b) Operations: The primary interface with operations is through the processing of maintenance work requests (MWRs). These are packages that provide coordination of removing equipment from service (e.g. tagging out by operators), providing or recording clearance by health physics and quality assurance groups, and identifying the groups) within maintenance (electrical, mechanical, etc.) that are responsible for performing the work. MWRs may be initiated by operations (for equipment failed in service or during operational testing) or by maintenance (scheduled maintenance or equipment failed during surveillance testing). MWR packages provide the major data items fro work by maintenance groups. MWRs are prioritized by their impact on safety availability and license conditions. It is not uncommon for minor items to be postponed by refueling outages without any direct influence on safety.

(c) Training: Training provides specialist skills, knowledge and practice to maintenance staff. Maintenance personnel are usually experienced in performing maintenance function on power plant equipment before they are recruited by a plant; such experience may have been gained from the nuclear navy or from fossil plants. The purpose of the plant-specific training is to acquaint personnel with new equipment, or with new techniques in maintenance such as the use of condition-monitoring equipment. Training needs may also be identified by evaluation of equipment and plant performance-see training interfaces below.

(d) Quality Assurance: The role of quality assurance is to provide an independent checker on the adequacy and completeness of work associated with plant safety. Its interface with maintenance is principally associated with specifying "hold and check" points during repair and restoration to service following maintenance. These may be specified as part of the MWR package or as a separate requirement.

(e) Plant Engineering/Support: The interface with the plant engineering/support group is concerned with the lessons learned from equipment failures and performanceespecially root cause analyses of problems. This information identifies implicitly needed changes in maintenance activities to avoid the repetition of plant problems.

(f) Purchasing/Construction: The interface is associated with describing maintenance requirements and practices associated with new equipment. It will provide (in parallel to training) manuals and other documentation associated with maintenance of new equipment; it may also include manufacturer-based training for certain types of new equipment. In addition, maintenance may provide requirement to purchasing/construction concerning the maintainability of planned purchases. 
(g) Physical Plant: The interfaces with the physical plant are the monitoring and repair of plant equipment. Monitoring includes both the formal testing and surveillance checks as well as the informal observations by maintenance personnel whilst in plant areas.

\section{Training}

(a) Operations: Training provides the operations group with specific skills rules and knowledge associated with all phases of the plant operations, including startup and shutdown, steady-state power control, refueling and emergency scenarios. Skills are acquired largely through practicing drills in training simulators; rules are provided in the form of operating procedures; and knowledge is provided through classroom instruction. Some fraction of operators joining a power plant have nuclear operating experience (either from the nuclear navy or from another plant) and therefore initial training achieves a plant orientation for others, training provides a more broad basic in nuclear technology as well as plant specific information. Once qualified operators are retrained on a provided basis, with emphasis on skill retention and knowledge associated with recent significant operating events.

In addition, operations many request specific training activities typically for new or difficult maneuvers, or because of identified performance problems. These training needs are from operations into training.

(b) Maintenance: See above.

(c) Site Management:--Site management's interface with training is primarily the allocation of goals and resources to the training group; these may include identified numbers of trainees and associated budgets.

(d) Plant Engineering/Support: This interface is to identify training needs directly into training as a result of identified problems in systems performance or, more frequently, because of changes in operating practices such as in response to new NRC requirements (as happened with the NRC's post-TMI action plan).

(e) Purchasing/Construction: As new equipment is purchased and installed, the purchasing/construction group may supply (or arrange the supply of) new training materials, manuals, etc. to the training group for incorporation in the training process. In addition, changes in the plant need to be represented in the training simulator, such as modifications to the simulator software, the panel layouts, and related system descriptions.

\section{Operations}

(a) Maintenance: See above

(b) Training: See above

(c) Site Management: The interface with site management is principally the goals and resources for operations (such as the number of operating shifts, the targeted availability and cumulative generation, and so on) and the reporting by operations of the commercial plant performance (daily during management meetings, and, more formally, on a weekly or monthly basis). 
(d) Quality Assurance: The interface between operations and quality assurance is the reporting checks of the status of safety systems through test schedules and control-room status logs.

(e) Performance Analysis: Performance analysis provides operations with summaries of systems performance. These summaries may include thermal-hydraulic data (flows, heat balances, etc.) and reliability data (trends in failures, etc.).

(f) Other Plant Functions: The primary interface with other plant groups is with health physics (HP) in requesting and receiving clearances for access to potentially or actually contaminated zones or other areas where significant radiation may be present. Operation may coordinate HP clearances for maintenance work (as part of the MWR process) as well as for operational access.

A secondary interface exists with security and safeguards to obtain access to designated plant areas (usually through a key card system, but sometimes through guard monitors--especially during refueling).

(g) Physical Plant Systems: Most of the day-to-day interactions by operations is with the plant hardware through controlling changes in plant-state, monitoring equipment performance and performing operability tests on safety-related equipment. Such activities are performed both from the control room, from local control stations and at the equipment locations.

\section{Quality Assurance}

(a) Corporate Management: Quality assurance normally reports to a senior manager (sometimes a QA designated vice president, sometimes the president directly) in the corporate management off-site. QA reports identified non-conformances and deficiencies in plant performance related to safety independently of the operationsmanagement chain, to provide a check-and-balance on plant administration.

(b) Site Management: Information sent by the QA group to the corporate management is usually provided to site management in parallel for information.

(c) Maintenance: See above

(d) Operations: See above

\section{Performance Analysis}

(a) Site Management: Site management provides the goals and resources for the performance analysis group. Often the performance analysis group is administratively part of another unit (plant engineering or operations) so this interface is comparatively weak.

(b) Operations: See above

(c) Plant Engineering/Support: Performance analysis provides the plant engineering/support group with summaries of equipment performance data that can be used as a basis to identify plant problęm areas for analysis.

(d) Physical Plant Systems: Performance analysis obtains information from logsheets or computer monitoring systems that periodically record equipment status, flows, etc. 
Log sheets may be obtained physically from operations or performance analysis personnel may take separate logs of the plant.

\section{Plant Engineering/Support}

(a) Site Management: Site management provides the goals and resources for the plant engineering/support group. These may include specific medium and long term operational problems requiring resolution, implementing new NRC requirements, etc. Plant engineering/support provides additional perspectives on plant performance to site (and thence to corporate management). These perspectives are usually more analytically oriented then those provided by operations, etc. For example, they would include analyses of significant events and trends in performance, and utility-specific performance indicators.

(b) Purchasing/Construction: The plant engineering support function identifies engineering requirements and specifications to the purchasing/construction group as an input to the purchasing process.

(c) Training: As above.

(d) Operations: As above.

(c) Maintenance: As above.

\section{Purchasing/Construction}

(a) Corporate Management: Purchasing/construction interfaces with corporate management principally as a means of obtaining funds for large-scale projects that exceed site management authorizations.

(b) Plant Engineering/Support: See above.

(c) Training: See above.

(d) Maintenance: See above.

(e) Operations: See above.

\section{Site Management}

(a) Other Plant Functions: As with the other site-management interfaces, this interface supplies the goals and resources to the other plant functions.

(b) Training: As above.

(c) Operations: As above.

(d) Performance Analysis: As above.

(e) Plant Engineering/Support: As above.

(f) Corporate Management: Corporate management provides the site management with the overall goals and resources of the utility, usually in the form of financial and operational goals and overall budgetary resources. Implicitly the corporate management 
provides the "cultural" environment for the utility, including the site management and staff.

In addition, site management provides the corporate management with information about the overall plant performance, including economic and safety-related data.

9. Other Plant Functions

(a) Physical Plant Systems: These interfaces are information concerning radiation and dose readings (HP), water quality, etc., (chemistry), access logs (safeguards), and so on. 
Appendix B, Detailed Results of Statistical Analysis 


\section{Detailed Results for Plant A}


Maintenance Indicators $v s$. Measures of Safety

\begin{tabular}{|c|c|c|c|c|c|c|c|c|c|c|c|c|c|}
\hline & RANK & 1SCRTL & 1 SCRHI & 15CRLO & $255 R$ & 3SE & 4SSF & SFDR & ZEFO & CRI THOUR & SALP-M & FRATE & RUAIL \\
\hline 1PMTOTM & $\mathrm{N} / \mathrm{A}$ & $N / A$ & $N / A$ & $M / A$ & $M / A$ & $N / A$ & $\mathrm{~N} / \mathrm{A}$ & $N / A$ & $N / A$ & $N / A$ & $M / A$ & $\mathrm{H} / \mathrm{B}$ & $N / A$ \\
\hline $2 \mathrm{CMB}$ & $N / A$ & $N / A$ & $N / A$ & $N / A$ & $N / A$ & $N / A$ & $\mathrm{~N} / \mathrm{A}$ & $N / A$ & $M / A$ & $M / A$ & $N / A$ & $N / A$ & $N / R$ \\
\hline 3PMDVER & $N / A$ & $N / R$ & $\mathrm{H} / \mathrm{A}$ & $N / A$ & $N / A$ & $N / A$ & $N / A$ & $N / A$ & $N / P$ & $N / A$ & $N / A$ & $N / A$ & $N / A$ \\
\hline 4MNTTO1 & $M / A$ & $N / A$ & $N / A$ & $N / A$ & $N / A$ & $N / A$ & $N / A$ & $N / A$ & $N / A$ & $N / A$ & $N / A$ & $N / A$ & $N / A$ \\
\hline 5REMDRK $1 *$ & $N / A$ & $N / A$ & $N / A$ & $N / A$ & $N A$ & $N / A$ & $M / R$ & $N / A$ & $N / A$ & $M / A$ & $N / R$ & $\mathrm{~N} / \mathrm{A}$ & $N / A$ \\
\hline GDEF IC & $N / A$ & $N / A$ & N/A & $N / A$ & $N / A$ & $N / R$ & $N / A$ & $N / R$ & $N / A$ & $N / A$ & $N / R$ & $\mathrm{~N} / \mathrm{A}$ & $N / A$ \\
\hline PCMWRPER & $N / A$ & $N / A$ & $M / A$ & $N / A$ & $N / A$ & $N / A$ & $N / A$ & $N / A$ & $N / R$ & $N / A$ & $N / A$ & $N / A$ & $N / A$ \\
\hline 7DBOPMT & $\mathrm{N} / \mathrm{A}$ & $\mathrm{N} / \mathrm{A}$ & $N / A$ & $N / A$ & $N / A$ & $N / A$ & $N / A$ & $N / R$ & $N / A$ & $H / A$ & $N / A$ & $N / A$ & $N / A$ \\
\hline SMTSERV* & NS & NS & NS & NS & NS & NS & NS & NS & NS & NS & $N / A$ & NS & NS \\
\hline CSCRAIA & [Zero & events in & relevant & time sce & le] & & & & & & $N / A$ & & \\
\hline BASSPI & $N / A$ & $N / A$ & $N / A$ & $N / A$ & $N / A$ & $N / A$ & $N / A$ & $N / A$ & $N / A$ & $N / A$ & $N / A$ & $\mathrm{~N} / \mathrm{A}$ & $N / A$ \\
\hline 8BSSPI & $N / A$ & $\mathrm{~N} / \mathrm{B}$ & $N / R$ & $N / A$ & $M / A$ & $N / A$ & $N / A$ & $N / A$ & $M / A$ & $M / A$ & $N / A$ & $M / A$ & $N / A$ \\
\hline 8CSSPI & N/A & $W / A$ & $N / A$ & $N / A$ & $N / A$ & $N / A$ & $N / A$ & $M / A$ & $N / A$ & $\mathbf{H} \boldsymbol{A}$ & $N / A$ & $N / A$ & $M / A$ \\
\hline 8DSSPI & [Zero & events in & relevant & time sce & $1 \in]$ & & & & & & $N / A$ & & \\
\hline BAHRWA & $N / A$ & $M / A$ & $N / A$ & $N / A$ & $N / A$ & $N A$ & $N / A$ & $N / A$ & $N / A$ & $N / A$ & $N / A$ & $N / A$ & $N / A$ \\
\hline BBHRHA & $N / A$ & $M / A$ & $N / A$ & $N / A$ & $M / A$ & $\mathrm{~N} / \mathrm{A}$ & $N / A$ & $N / A$ & $N / A$ & N/A & $N / A$ & $M / A$ & $N / A$ \\
\hline BCHRNA & $N / A$ & $N / R$ & $\mathrm{H} / \mathrm{A}$ & $N / A$ & $N / A$ & $\mathrm{~N} / \mathrm{A}$ & $N / A$ & $N / A$ & $N / A$ & $N / R$ & $N / A$ & $N / P$ & $N / A$ \\
\hline BDHRNA & $N / A$ & $\mathrm{~N} / \mathrm{F}$ & $\mathrm{N} / \mathrm{A}$ & $N / A$ & $\mathrm{H} / \mathrm{A}$ & $N / A$ & $N / A$ & $N / A$ & N/A & $N / A$ & N/A & $N / A$ & $N / A$ \\
\hline BEHRIA & N/A & $N / R$ & $M / A$ & $N / A$ & $N / A$ & $N / A$ & $N / A$ & $N / A$ & $N / A$ & $N / A$ & N/A & $N / A$ & $N / A$ \\
\hline BFHRNA & $N / A$ & $N / A$ & $N / A$ & $N / A$ & $N / A$ & $M / A$ & $N / A$ & $N / A$ & $N / A$ & $N / A$ & $N / A$ & $N / A$ & $N / A$ \\
\hline BGHRNA & $N / A$ & N/A & $N / A$ & $N / A$ & $N / R$ & $N / A$ & $N / A$ & $N / A$ & $N / A$ & $N / R$ & $N / A$ & $N / A$ & $N / R$ \\
\hline CBSCRAM & $N / A$ & $N / R$ & $N / A$ & $N / A$ & $N / A$ & $N / R$ & $N / A$ & $N ノ A$ & $N / A$ & $M / A$ & $M / A$ & $N / A$ & $N / A$ \\
\hline GHR* & NS & NS & NS & NS & NS & NS & 5 & 5 & NS & NS & $N / A$ & 5 & NS \\
\hline WPE $\approx$ & NS & NS & N5 & NS & NS & NS & NS & MS & NS & MS & $N / A$ & NS & NS \\
\hline RERLE $*$ & [zero & events in & relevant & time sca & lej & & & & & & $N / A$ & & \\
\hline BACKECN* & $M / A$ & $N / A$ & $N / A$ & $\mathrm{~N} / \mathrm{A}$ & $W / A$ & $N / A$ & $\mathrm{~N} / \mathrm{A}$ & $N / A$ & $N / A$ & $N / A$ & $N / R$ & $N / A$ & $N / A$ \\
\hline WRONGT /L* & $N / A$ & $\mathrm{~N} / \mathrm{A}$ & $N / P$ & $N / A$ & $N / A$ & $N / A$ & $N / A$ & $N / A$ & $N / A$ & $N / A$ & $N / A$ & $N / A$ & $N / A$ \\
\hline ESFACT* & NS & NS & NS & NS & NS & NS & NS & NS & NS & NS & $N / R$ & NS & 5 \\
\hline
\end{tabular}


Significant Maintenance Indicator/Measure of Safety Relationships

\begin{tabular}{|c|c|c|c|}
\hline $\begin{array}{l}\text { Indicator } \\
======== \\
\text { ESFACT }\end{array}$ & $\begin{array}{l}\text { Measure of Safety } \\
================= \\
\text { RUAIL }\end{array}$ & $\begin{array}{l}\text { Time Shift } \\
\begin{array}{c}======= \\
+1\end{array}\end{array}$ & $\begin{array}{c}\text { Confidence } \\
======== \\
80 \%\end{array}$ \\
\hline GHR & $55 F$ & 0 & $82 \%$ \\
\hline GHR & EFD & 0 & $96 \%$ \\
\hline GHR & FRATE & 0 & $90 \%$ \\
\hline
\end{tabular}





B-5 

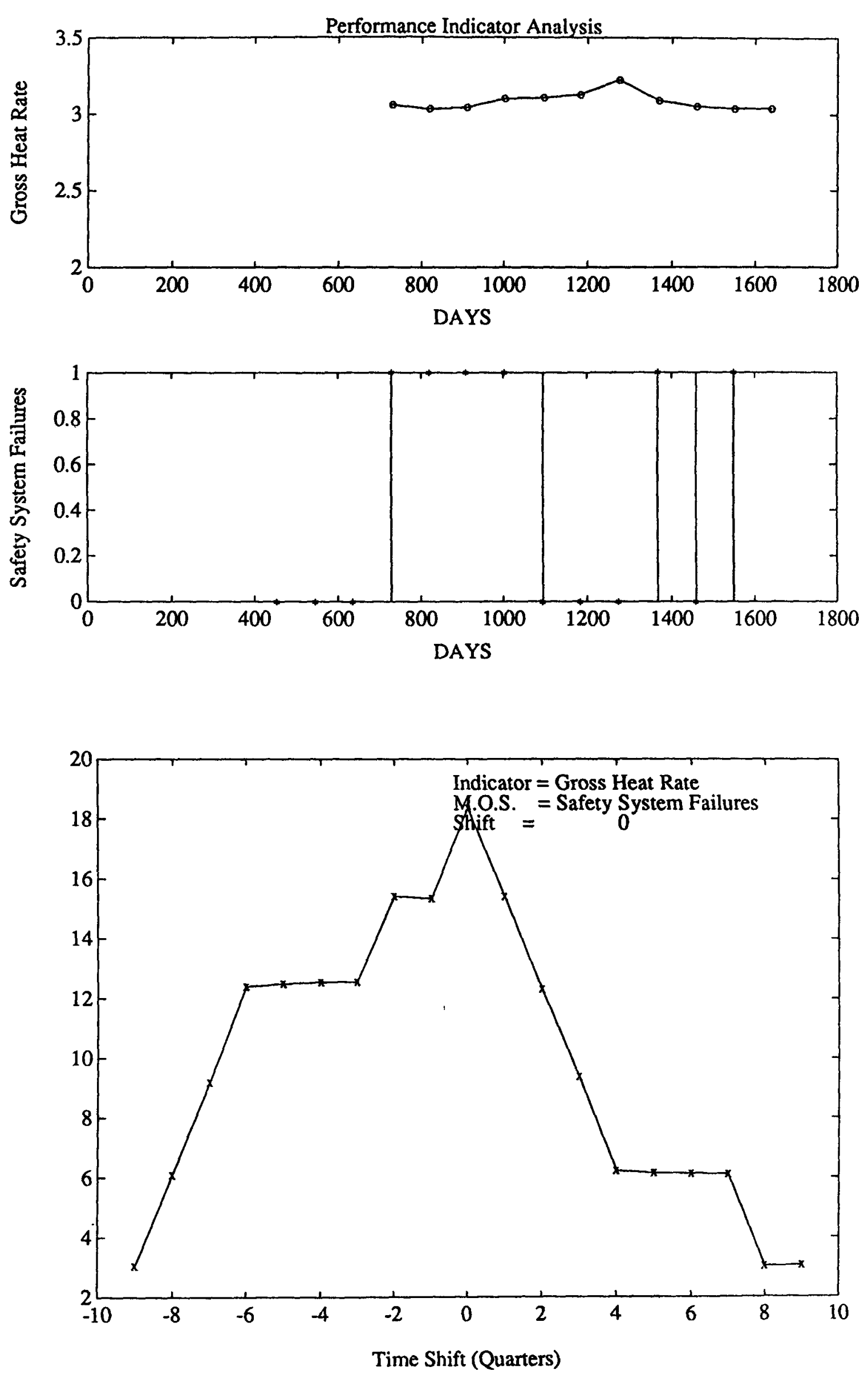

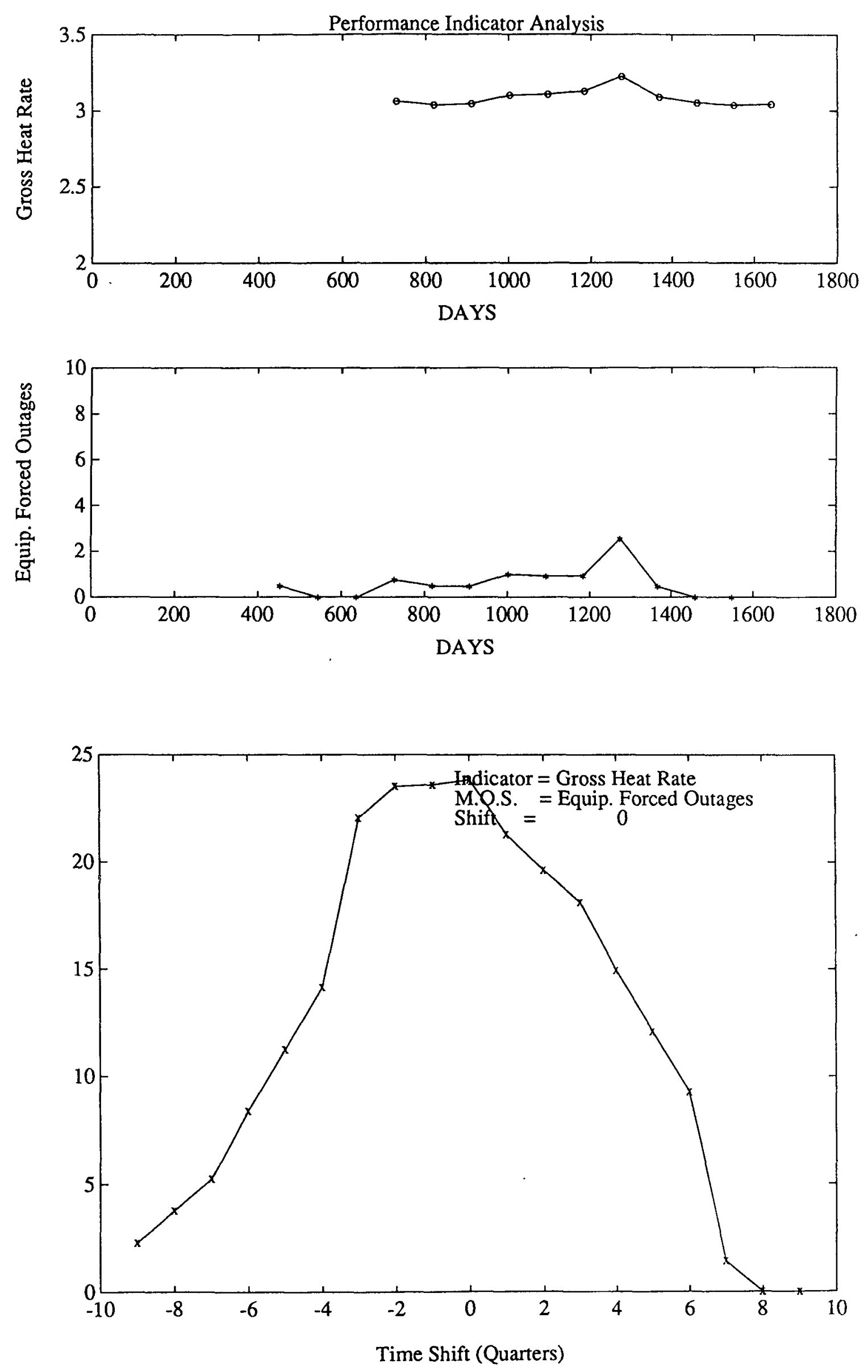

B-7 

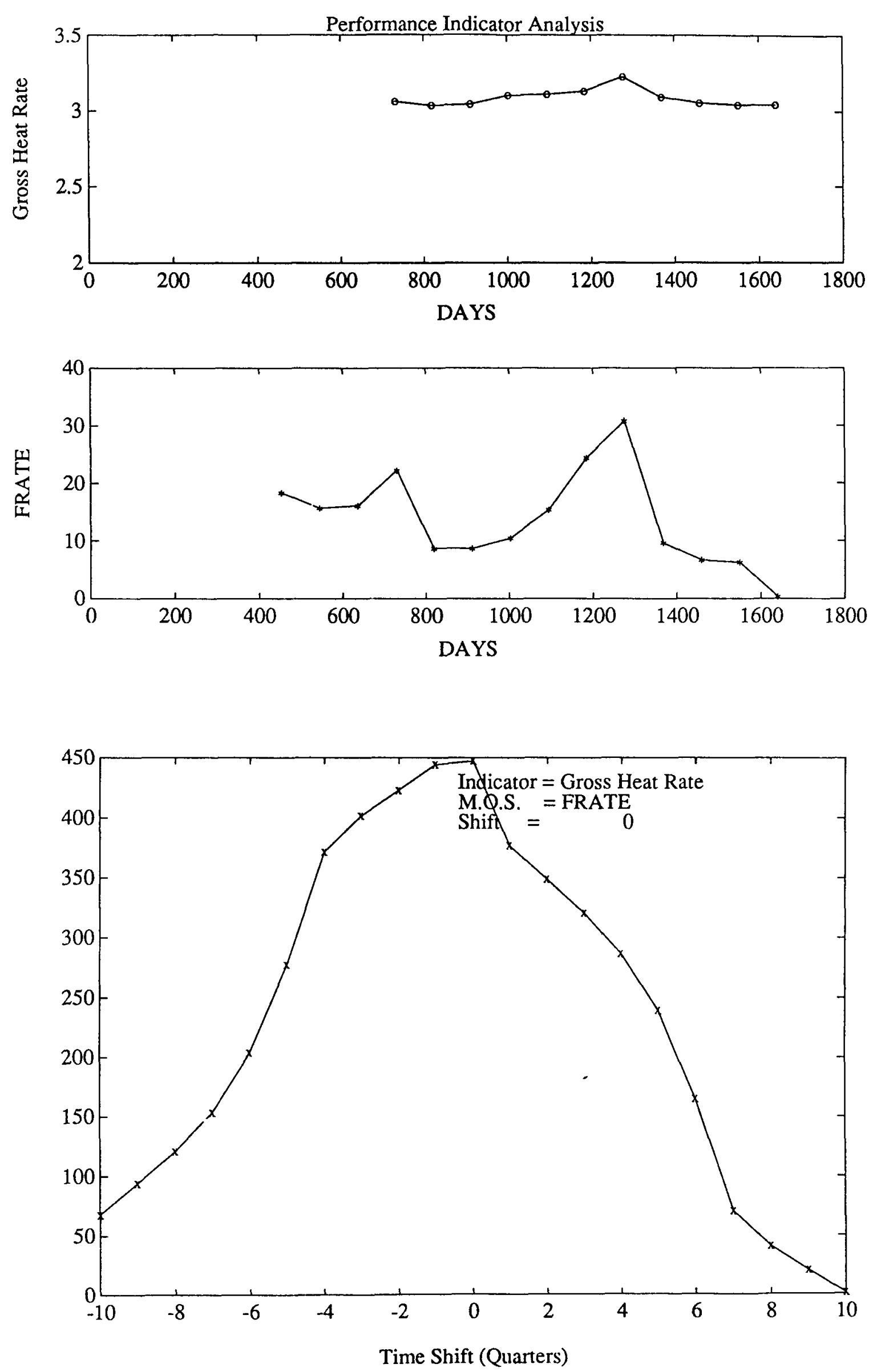

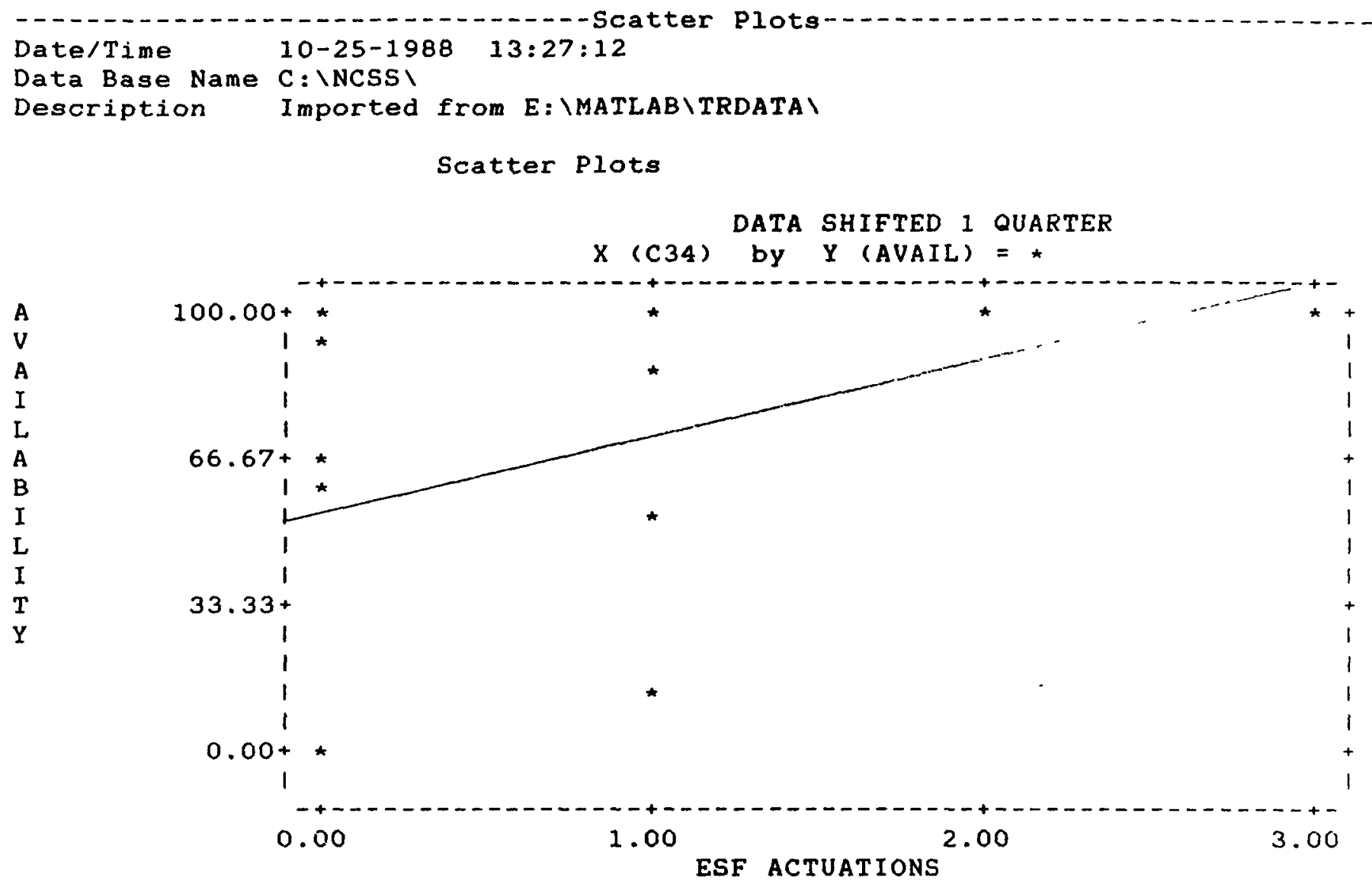

w Accounting Report

$\begin{array}{llllll}\text { Number of points below minimum } & (X): & 0 & (Y): & 0 \\ \text { Number of points above maximum } & (X): & 0 & (Y): & 0 \\ \text { Number of points with missing values }(X): & 1 & (Y): & 0 \\ \text { Number of points plotted } & & : & 13 & \end{array}$




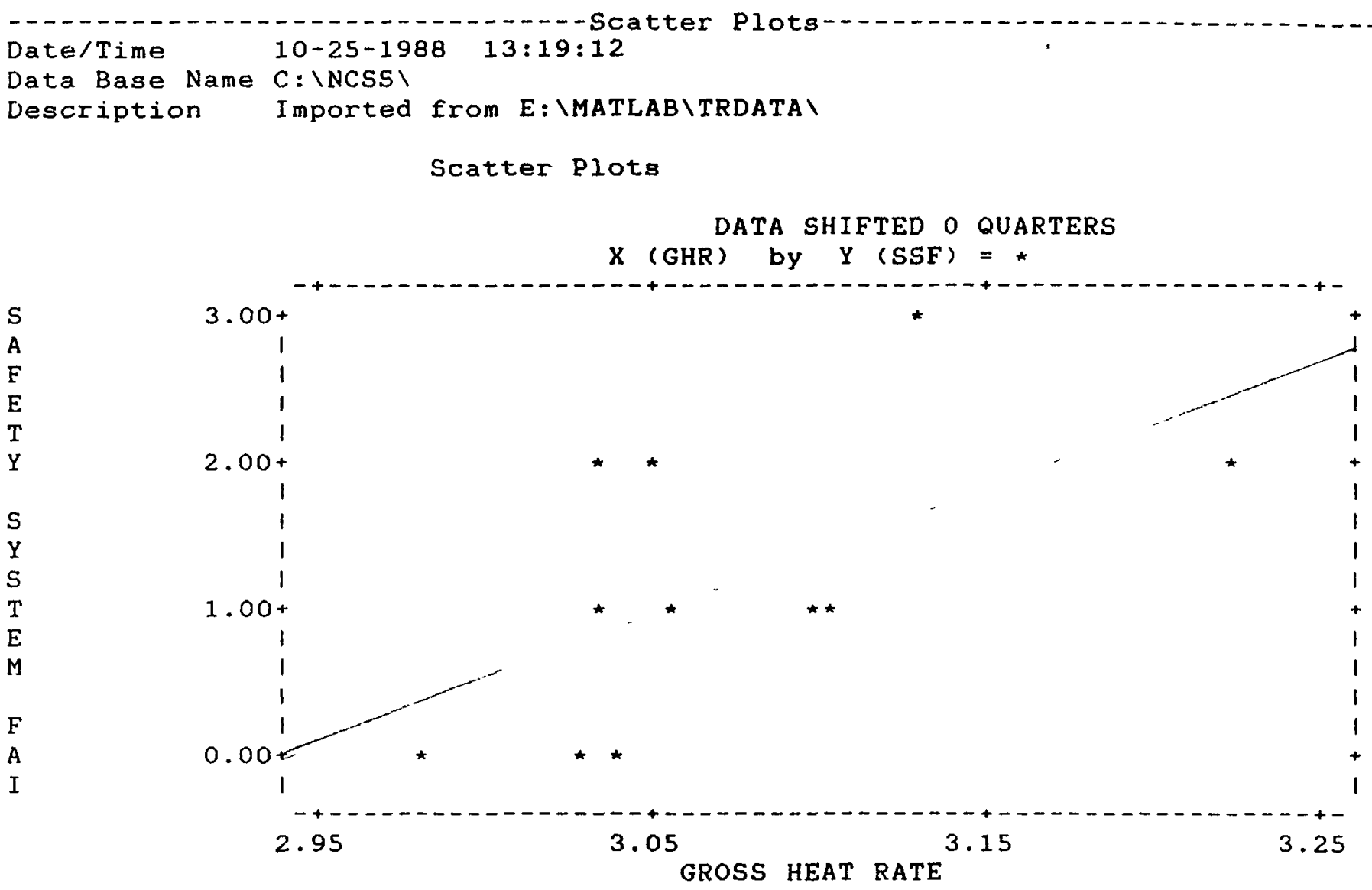

w Accounting Report

$\begin{array}{lllrrr}\text { Number of points below minimum } & (X): & 0 & (Y): & 0 \\ \text { Number of points above maximum } & (X): & 0 & (Y): & 0 \\ \text { Number of points with missing values } & (X): & 2 & (Y): & 1 \\ \text { Number of points plotted } & & : & 11 & & \end{array}$






DATA SHIFTED O QUARTERS

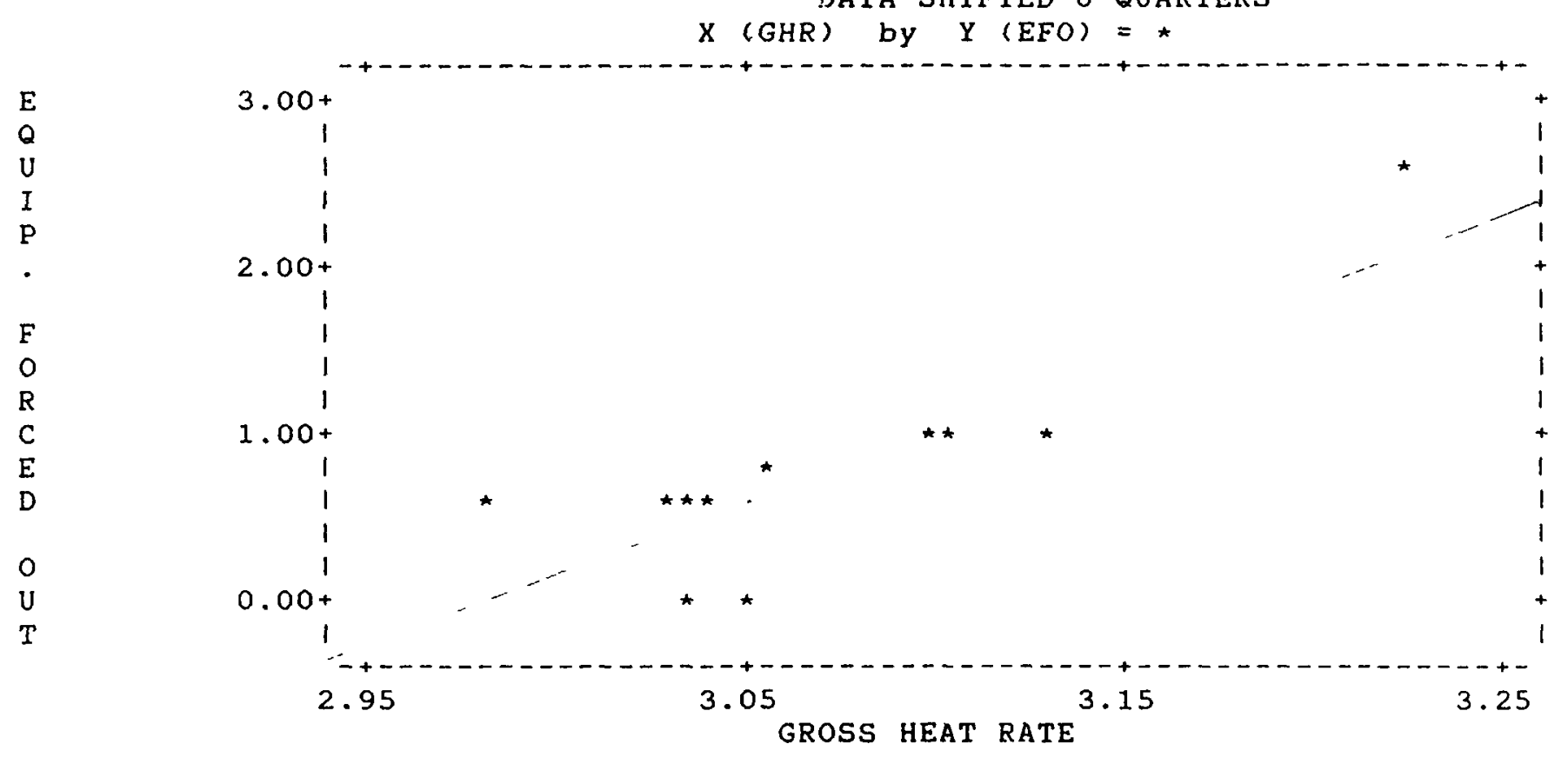

kow Accounting Report

Number of points below minimum Number of points above maximum Number of points with missing values Number of points plotted

\begin{tabular}{|c|c|c|c|c|}
\hline$(x)$ & : & 0 & $(Y)$ & : \\
\hline$(X)$ & : & 0 & (Y) & : \\
\hline$(X)$ & : & 2 & $(Y)$ & : \\
\hline & : & 11 & & \\
\hline
\end{tabular}




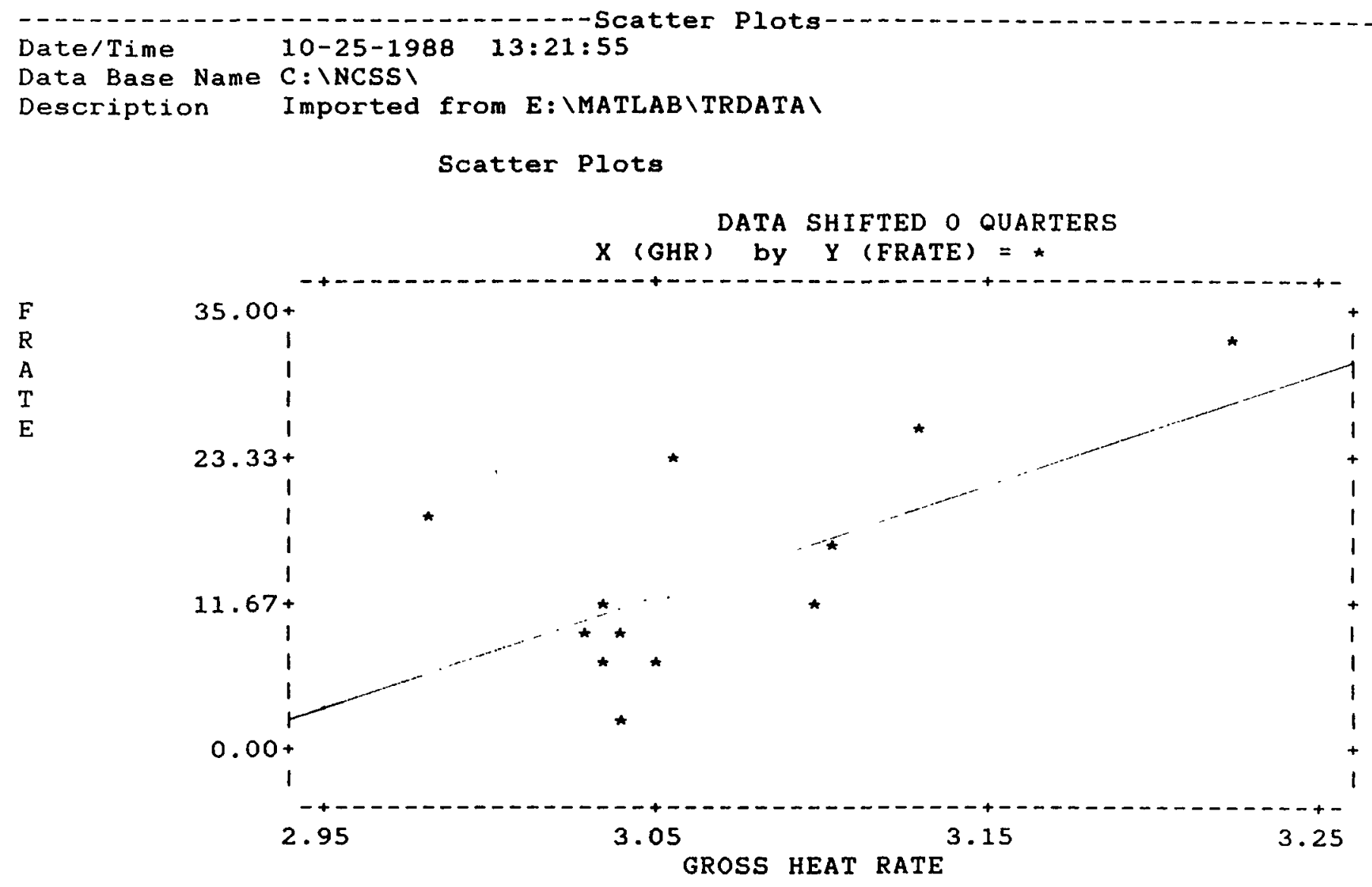

now Accounting Report

$\begin{array}{llrrrr}\text { Number of points below minimum } & (X): & 0 & (Y): & 0 \\ \text { Number of points above maximum } & (X): & 0 & (Y): & 0 \\ \text { Number of points with missing values } & (X): & 2 & (Y): & 0 \\ \text { Number of points plotted } & & : & 12 & & \end{array}$


Detailed Results for Plant B

B-13 
Maintenance Indicators vs. Measures of Safety

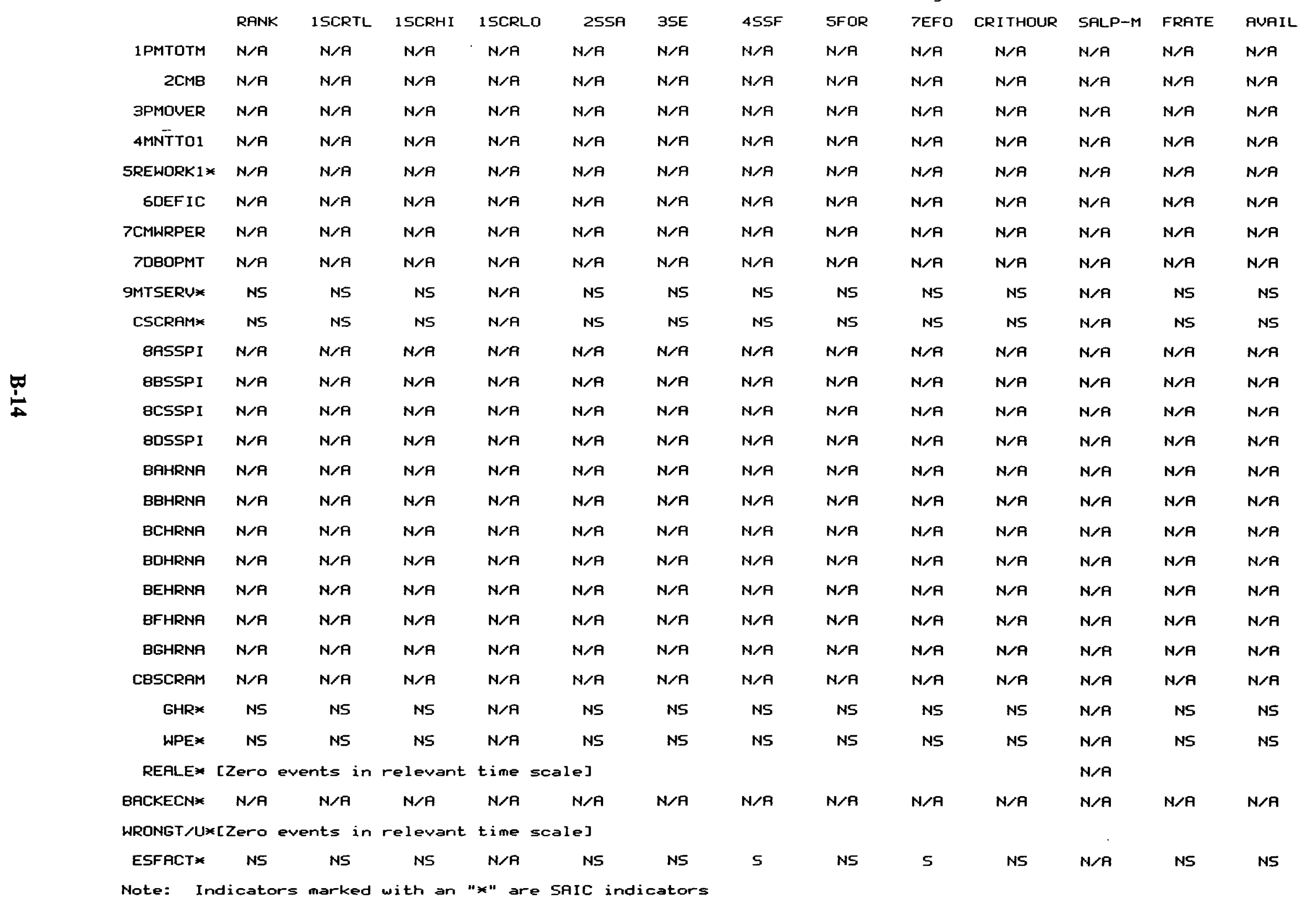


Significant Maintenance Indicator/Measure of Safety Relationships

\begin{abstract}
Indicator $=======$ ESFRCT
\end{abstract}

ESFACT

\section{Measure of Safety} = = = = = = = = = = = = = = = SSF

EFO
Time Shift $======$ $+6$

$+6$
Conf i dence $=======$ $94 \%$

89\% 

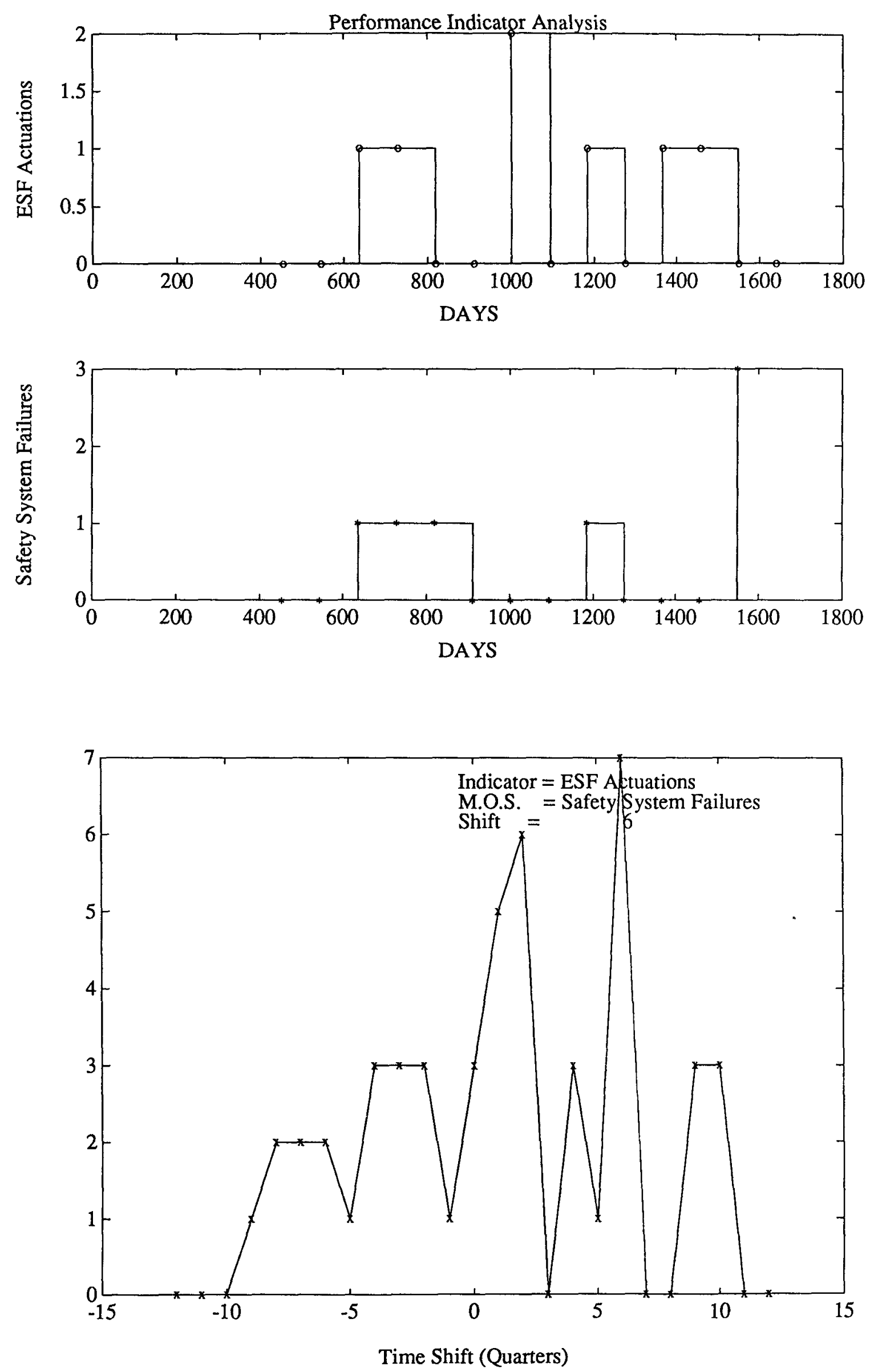

\section{B-16}



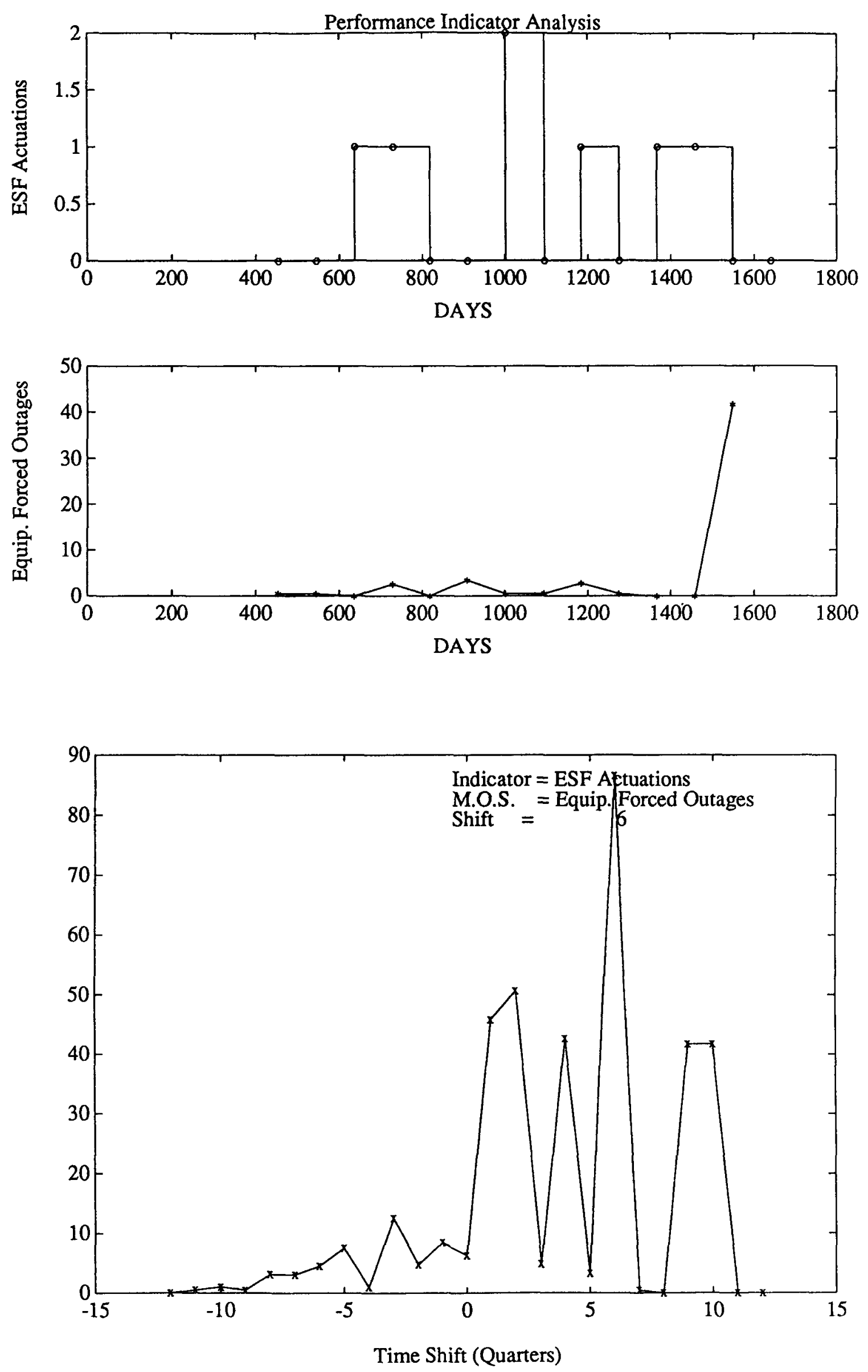

B-17 


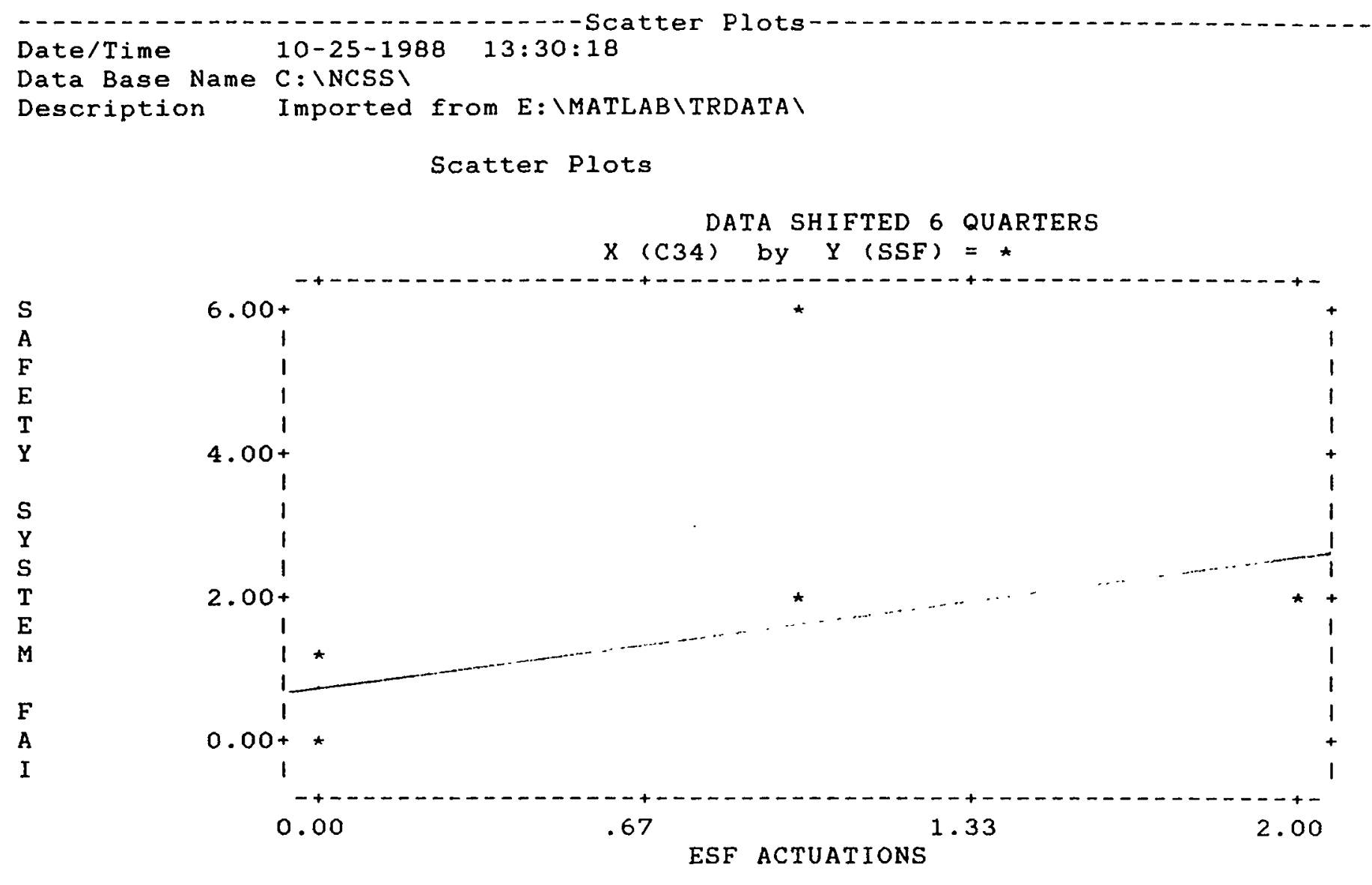

tw Accounting Report

$\begin{array}{lllllll}\text { Number of points below minimum } & (X): & 0 & (Y): & 0 \\ \text { Number of points above maximum } & (X): & 0 & (Y): & 0 \\ \text { Number of points with missing values }(X): & 6 & (Y): & 1 \\ \text { Number of points plotted } & & : & 7 & & & \end{array}$




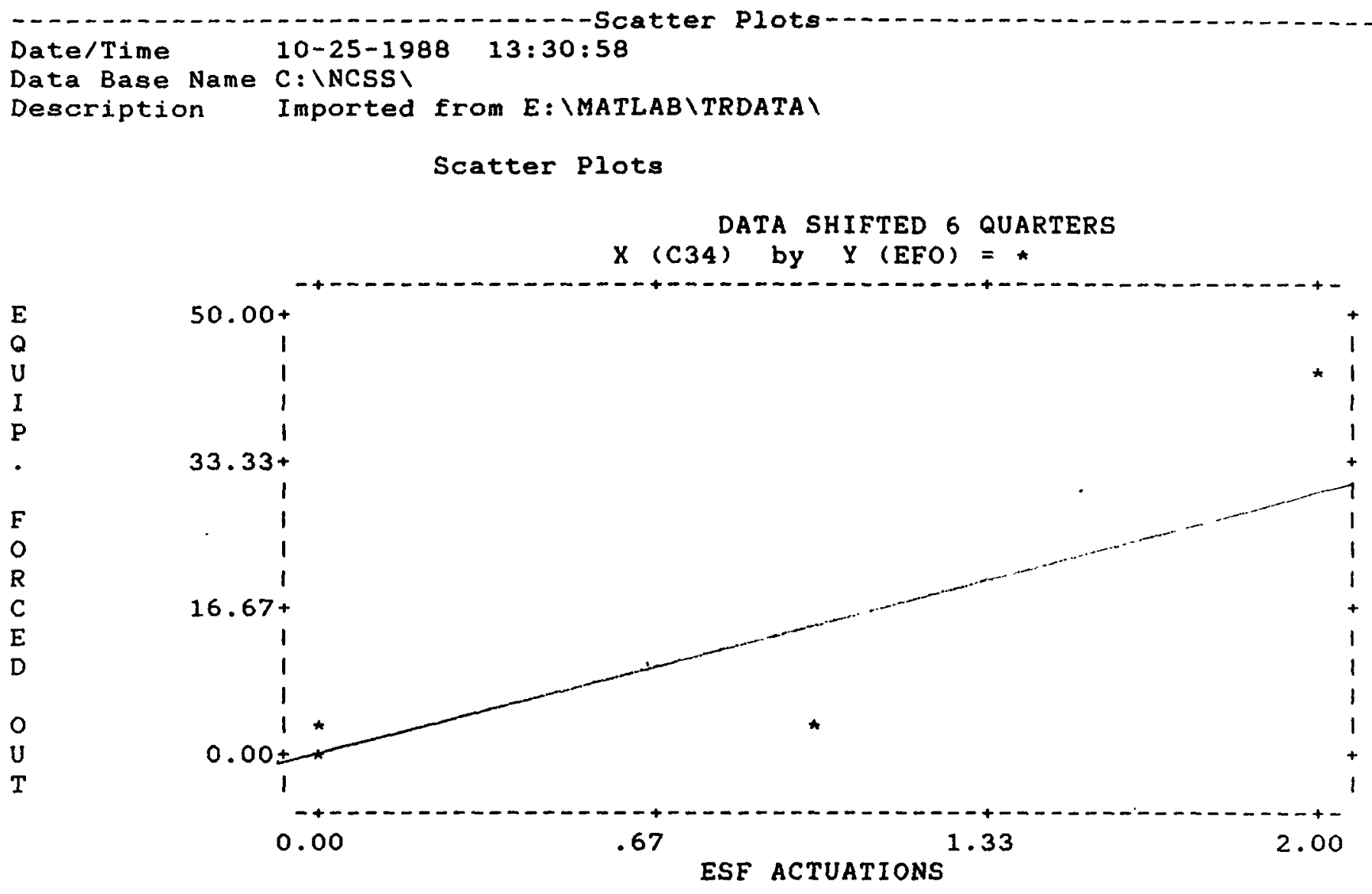

- uw Accounting Report

Number of points below minimum $\quad(X):=0$ (Y) : 0

Number of points above maximum $\quad(X): \quad 0 \quad(Y):=0$

$\begin{array}{lllll}\text { Number of points with missing values }(X): & 6 & (Y): & 1 \\ \text { Number of points plotted } & : & 7 & & \end{array}$ 


\section{Detailed Results for Plant C}


Maintenance Indicators $v s$. Measures of Safety

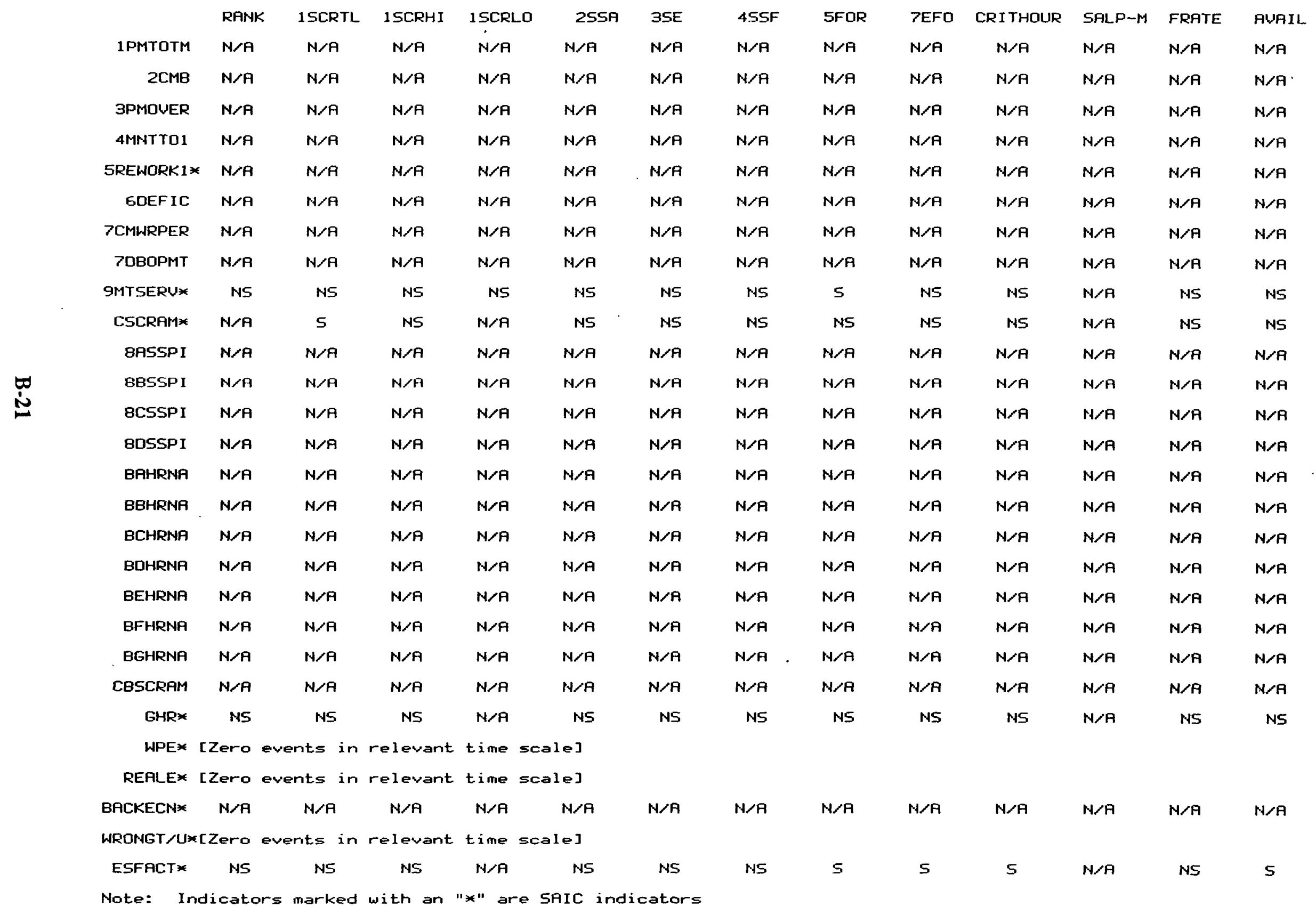


Significant Maintenance Indicator/Measure of Safety Relationships

\begin{tabular}{|c|c|c|c|}
\hline $\begin{array}{l}\text { Indicator } \\
=======\end{array}$ & $\begin{array}{l}\text { Measure of Safety } \\
==============\end{array}$ & $\begin{array}{l}\text { Time Shift } \\
========\end{array}$ & $\begin{array}{l}\text { Conf i dence } \\
=======\end{array}$ \\
\hline ESFACT & FOR & +2 & $80 \%$ \\
\hline ESFACT & EFO & +2 & $80 \%$ \\
\hline ESFACT & RUAIL & +4 & $89 \%$ \\
\hline ESFACT & CRIT & +4 & $91 \%$ \\
\hline SMTSERV & FOR & +4 & $87 \%$ \\
\hline CSCRAM & SCRTL & +3 & $90 \%$ \\
\hline
\end{tabular}



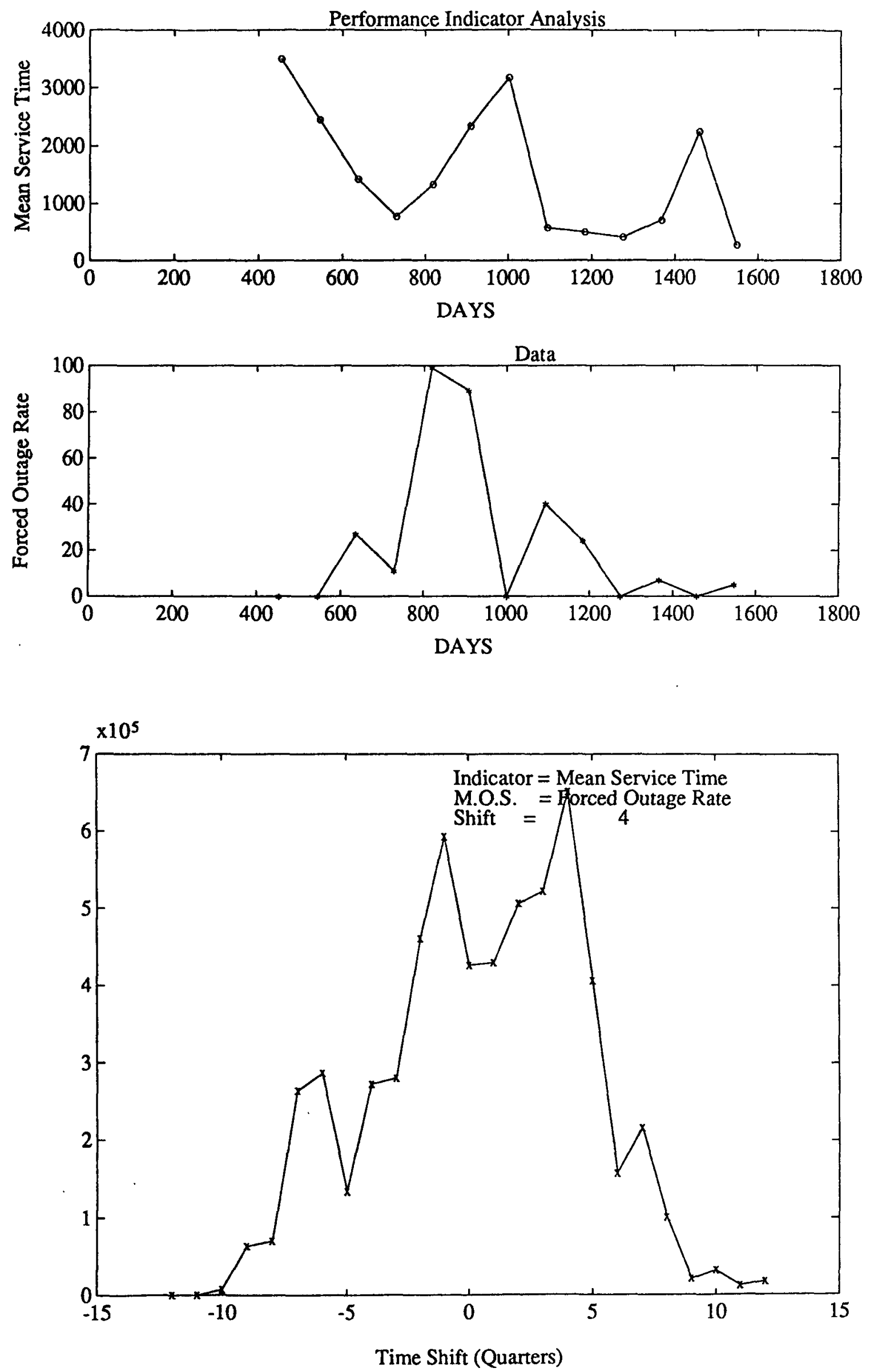

B-23 

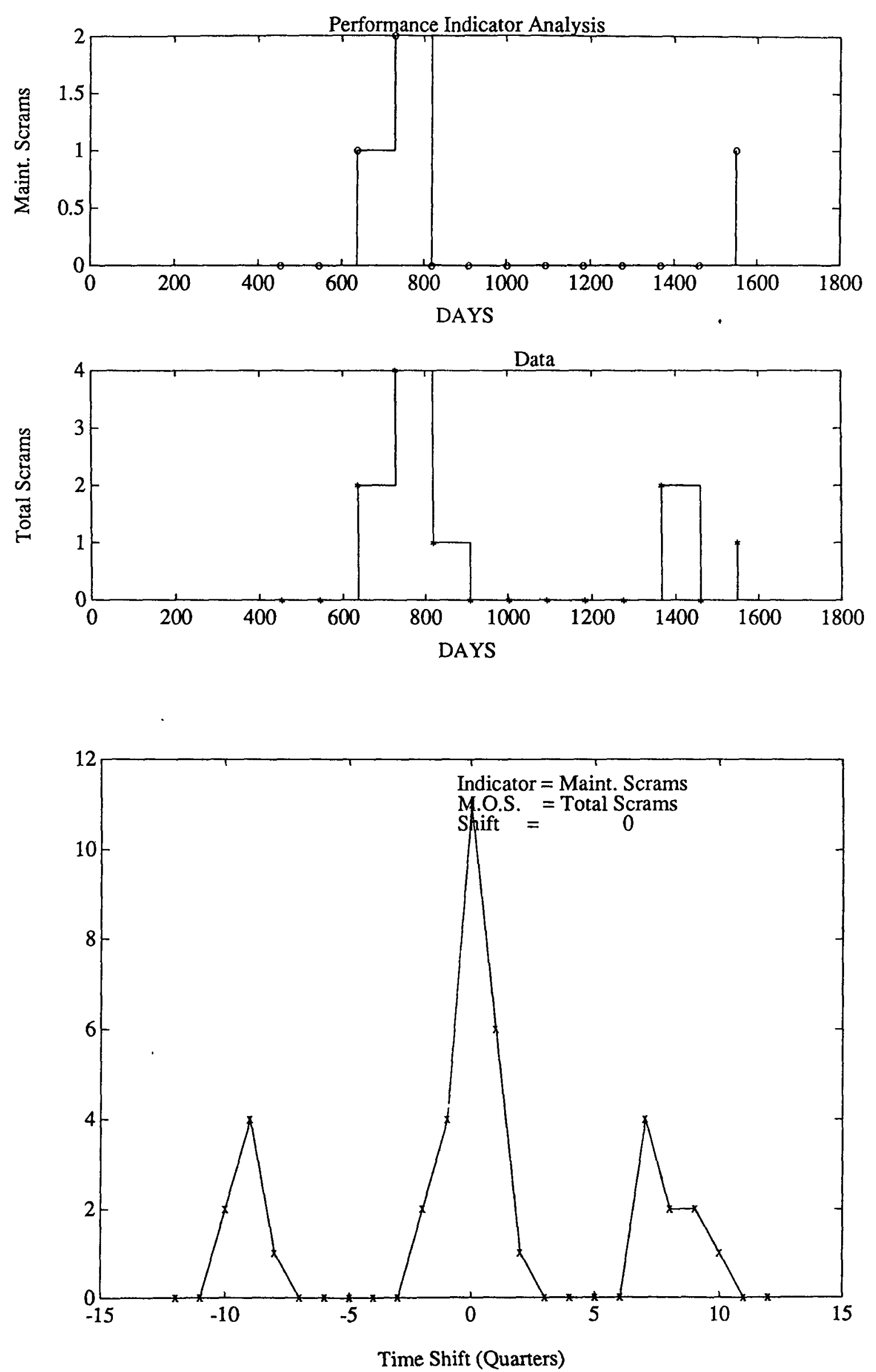

B-24 

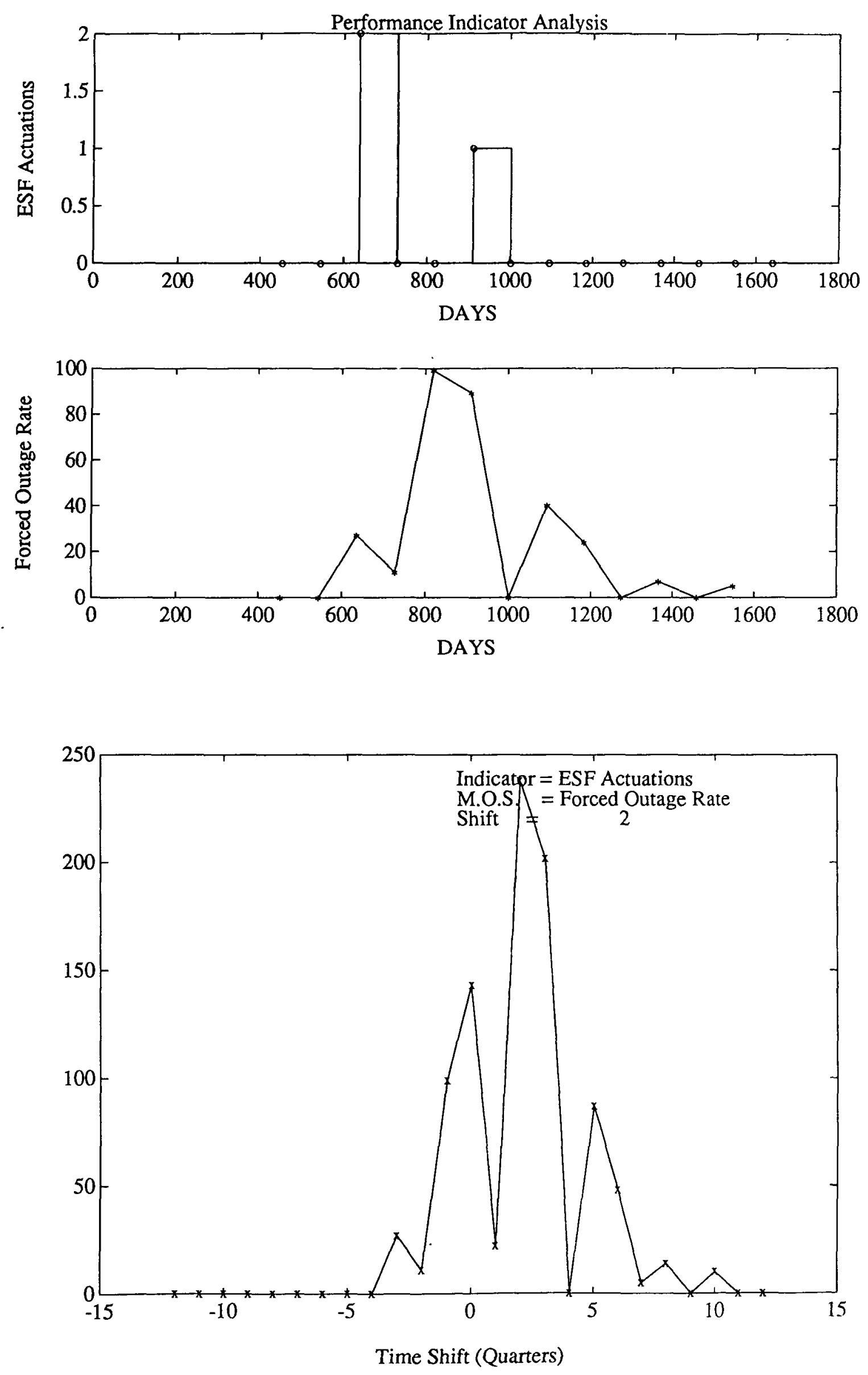

B-25 

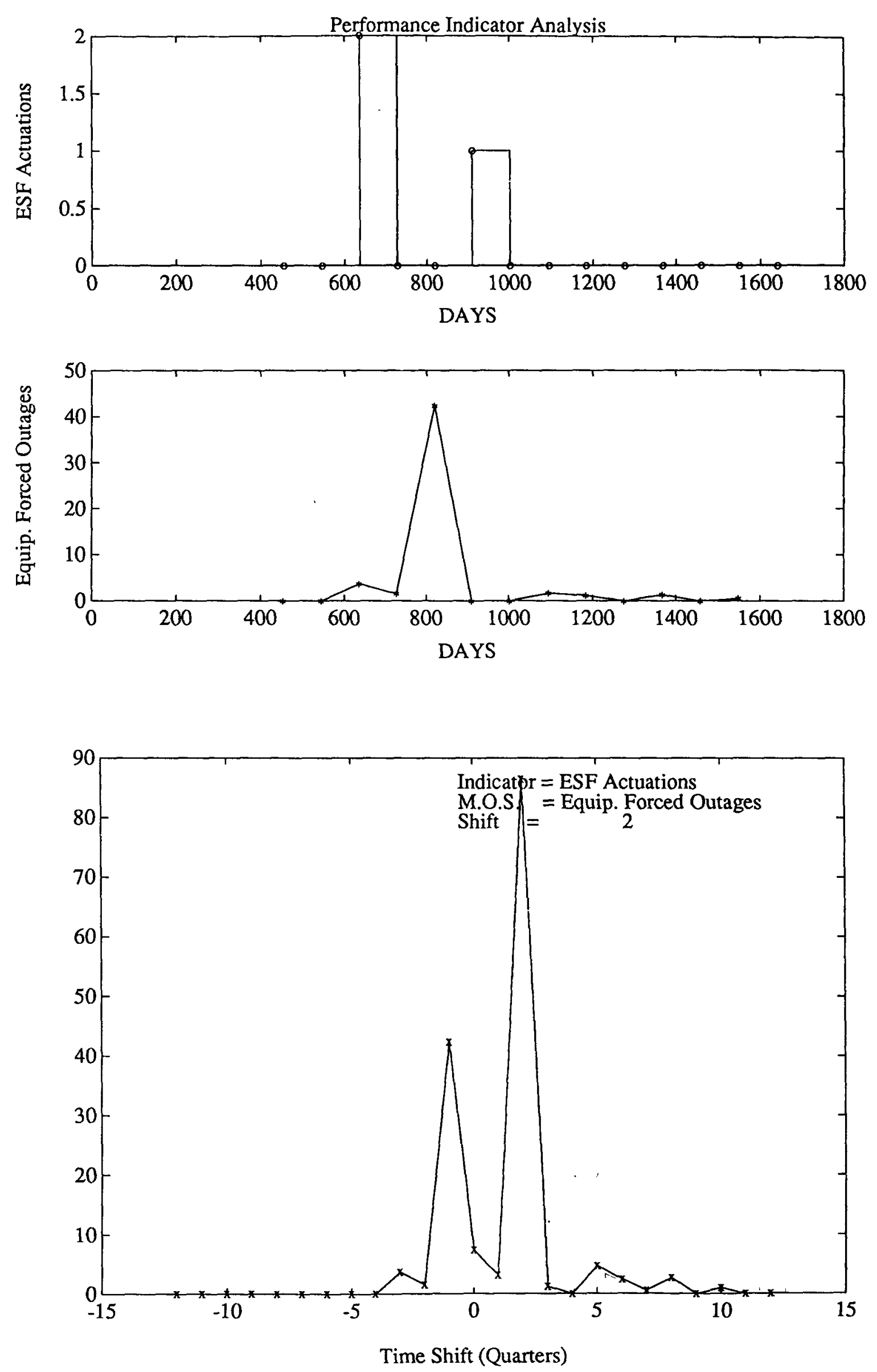

B-26 

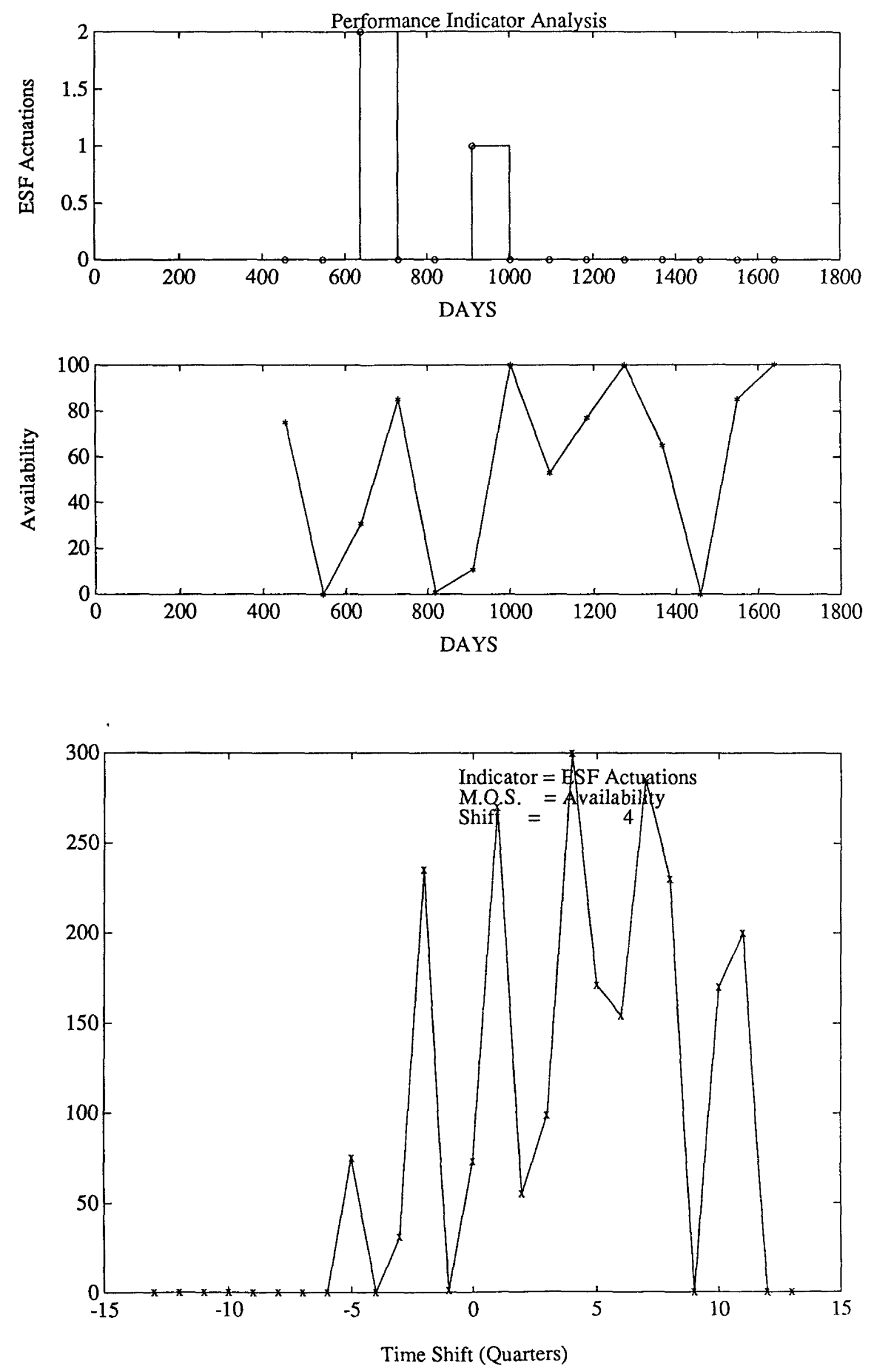

B-27 

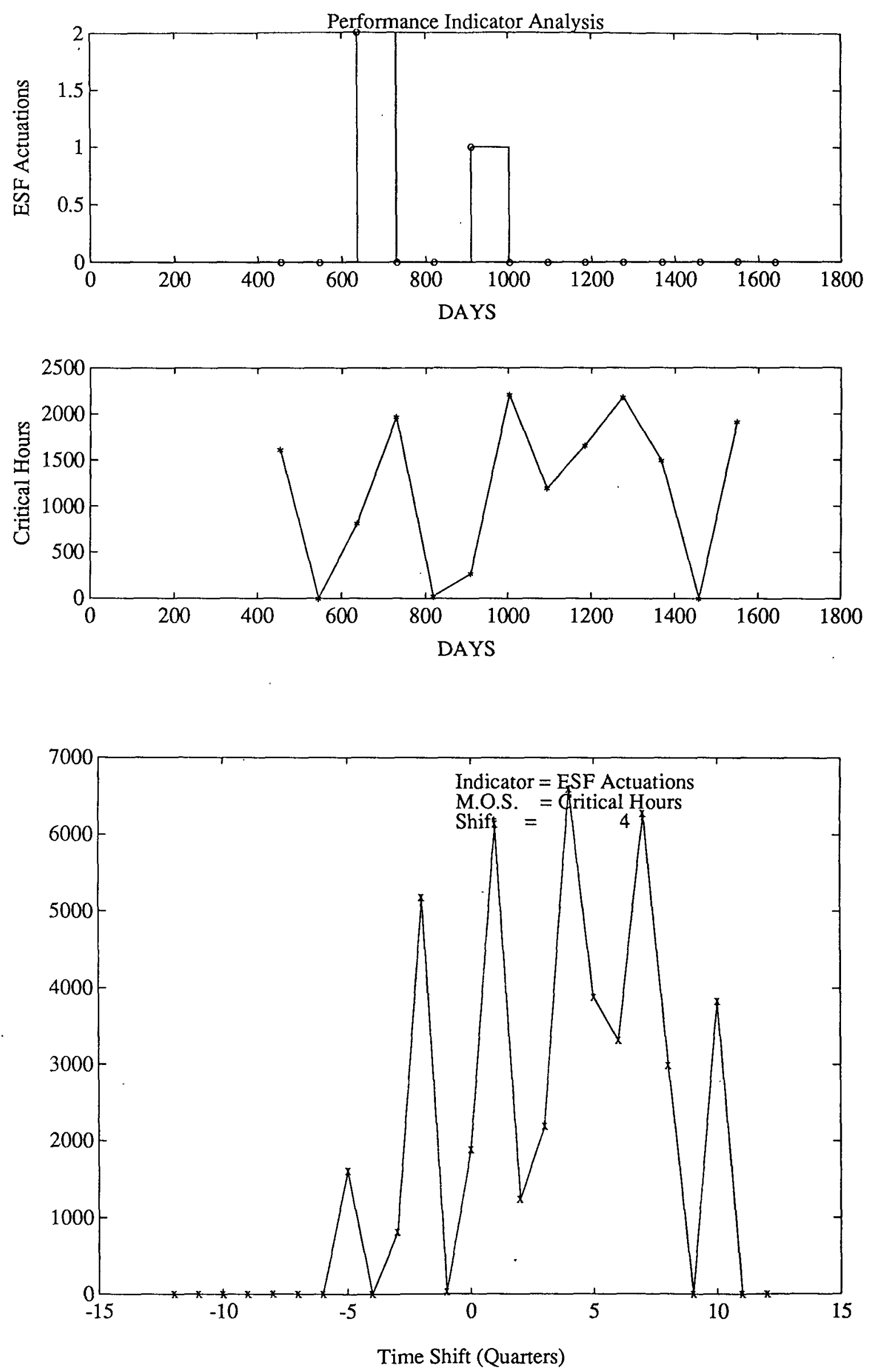

B-28 


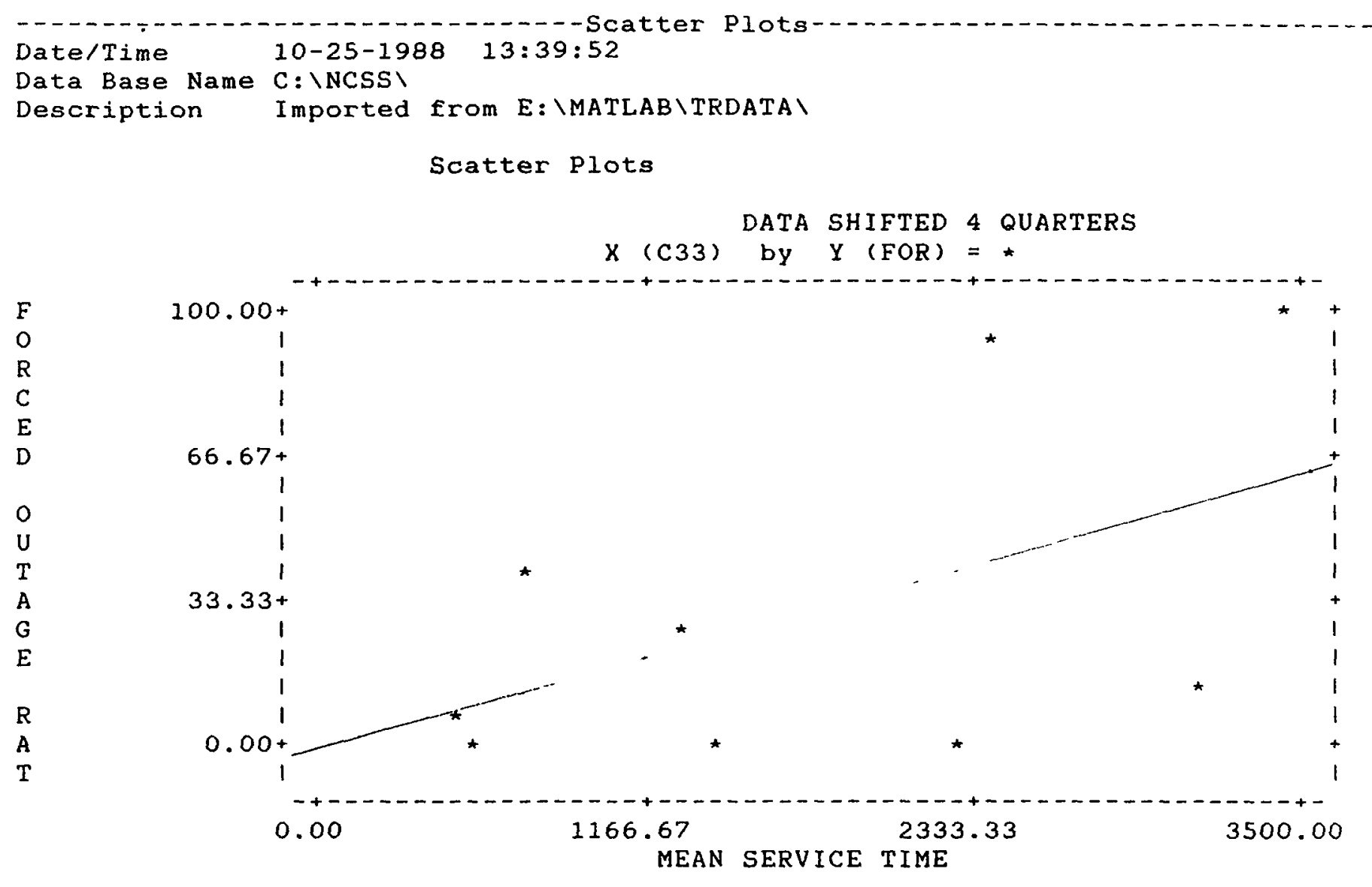

1. w Accounting Report

$\begin{array}{lllllll}\text { Number of points below minimum } & (X): & 0 & (Y): & 0 \\ \text { Number of points above maximum } & (X): & 0 & (Y): & 0 \\ \text { Number of points with missing values } & (X): & 4 & (Y): & 1 \\ \text { Number of points plotted } & & : & 9 & & & \end{array}$




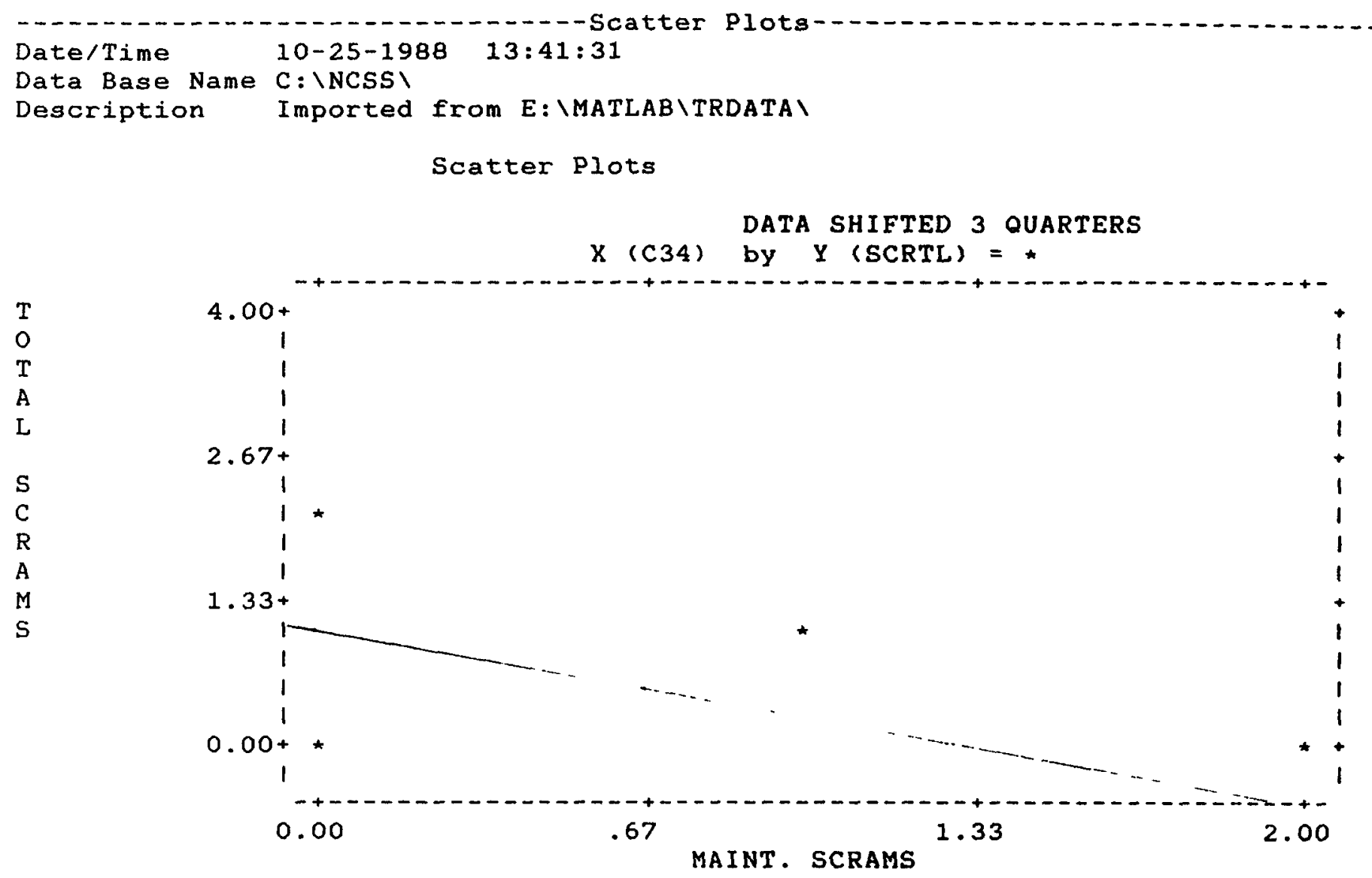

-w Accounting Report

$\begin{array}{llllll}\text { Number of points below minimum } & (X): & 0 & (Y): & 0 \\ \text { Number of points above maximum } & (X): & 0 & (Y): & 0 \\ \text { Number of points with missing values }(X): & 7 & (Y): & 1 \\ \text { Number of points plotted } & & : & 6 & & \end{array}$




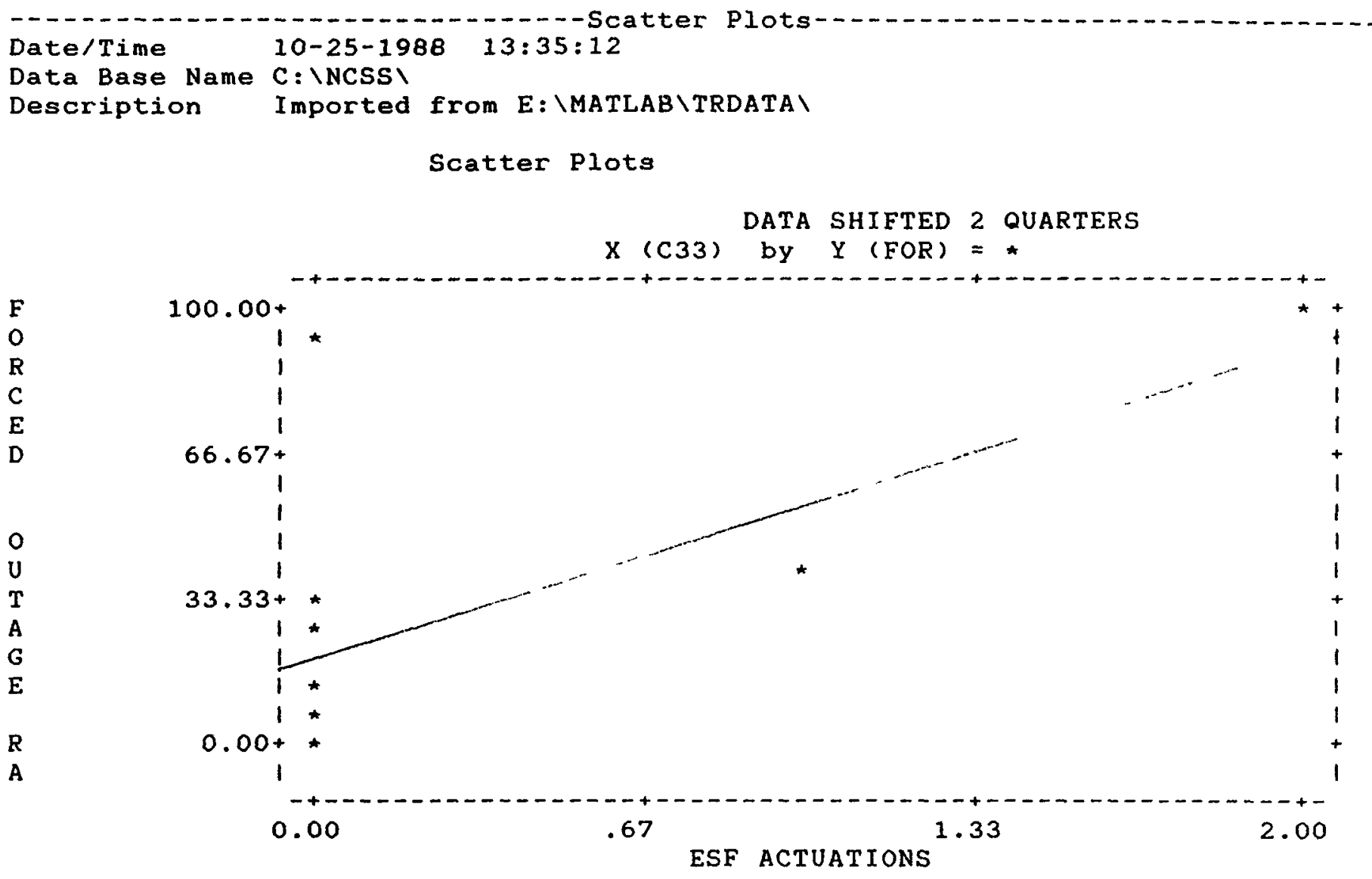

w Accounting Report

$\begin{array}{llrrrr}\text { Number of points below minimum } & (X): & 0 & (Y): & 0 \\ \text { Number of points above maximum } & (X): & 0 & (Y): & 0 \\ \text { Number of points with missing values } & (X): & 2 & (Y): & 1 \\ \text { Number of points plotted } & & : & 11 & & \end{array}$




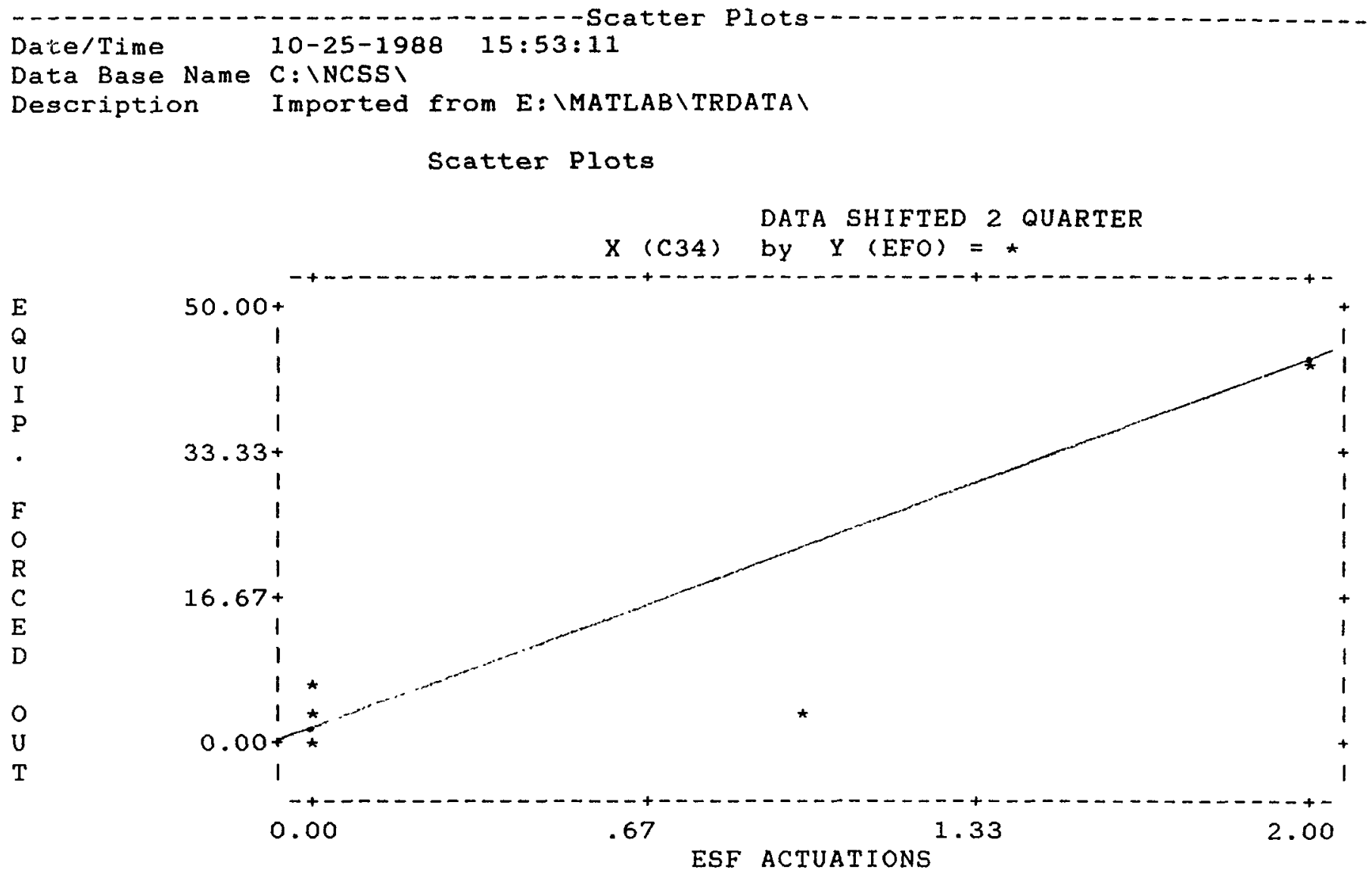

w Accounting Report

$\begin{array}{lllrl}\text { Number of points below minimum } & (X): & 0 & (Y): & 0 \\ \text { Number of points above maximum } & (X): & 0 & (Y): & 0 \\ \text { Number of points with missing values }(X): & 2 & (Y): & 1 \\ \text { Number of points plotted } & : & 11 & & \end{array}$




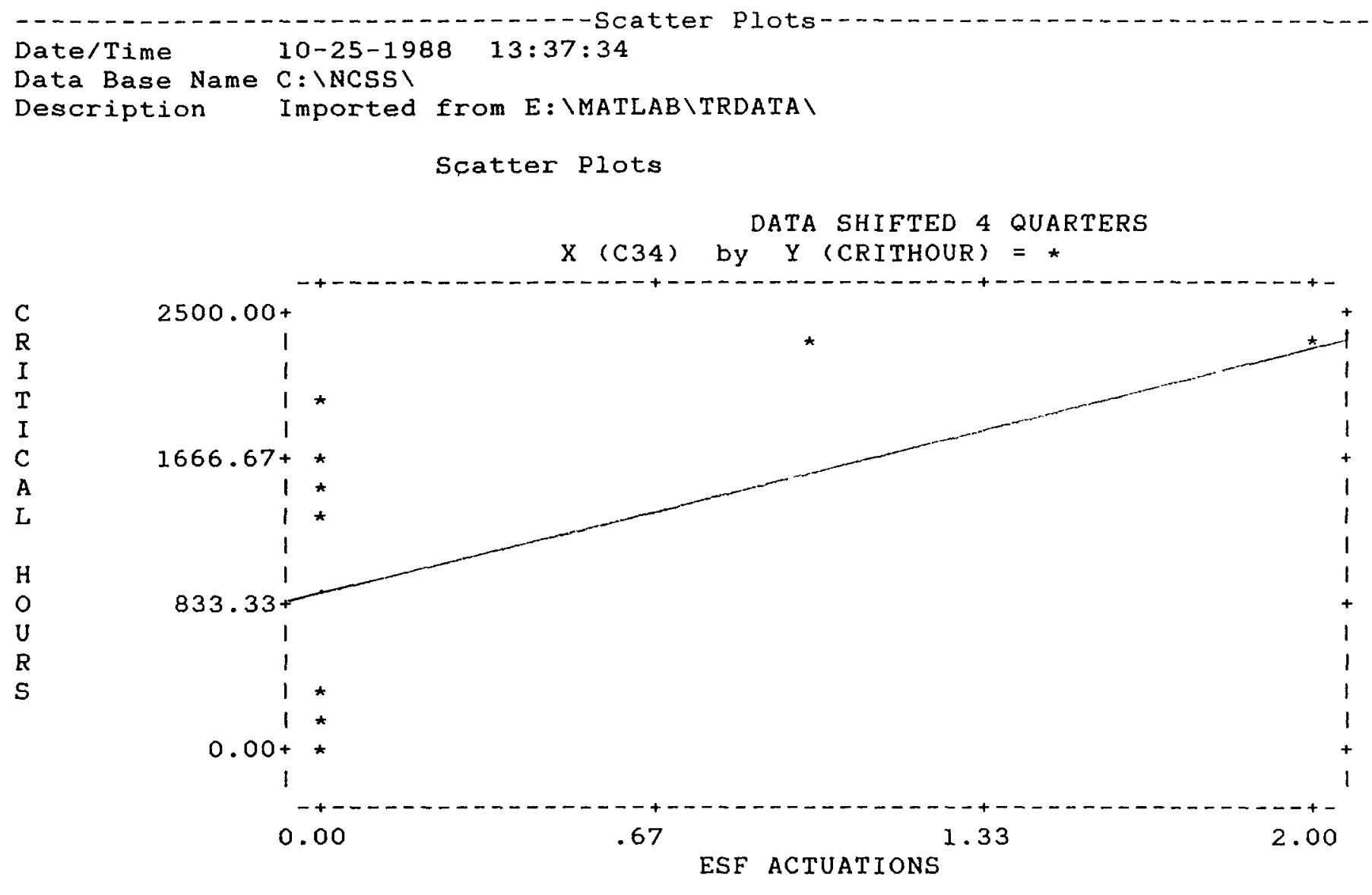

1. A Accounting Report

$\begin{array}{lllllll}\text { Number of points below minimum } & (X): & 0 & (Y): & 0 \\ \text { Number of points above maximum } & (X): & 0 & (Y): & 0 \\ \text { Number of points with missing values }(X): & 4 & (Y): & 1 \\ \text { Number of points plotted } & & : & 9 & & & \end{array}$




\section{Detailed Results for Plant E}


Maintenance Indicators vs. Measures of Safety

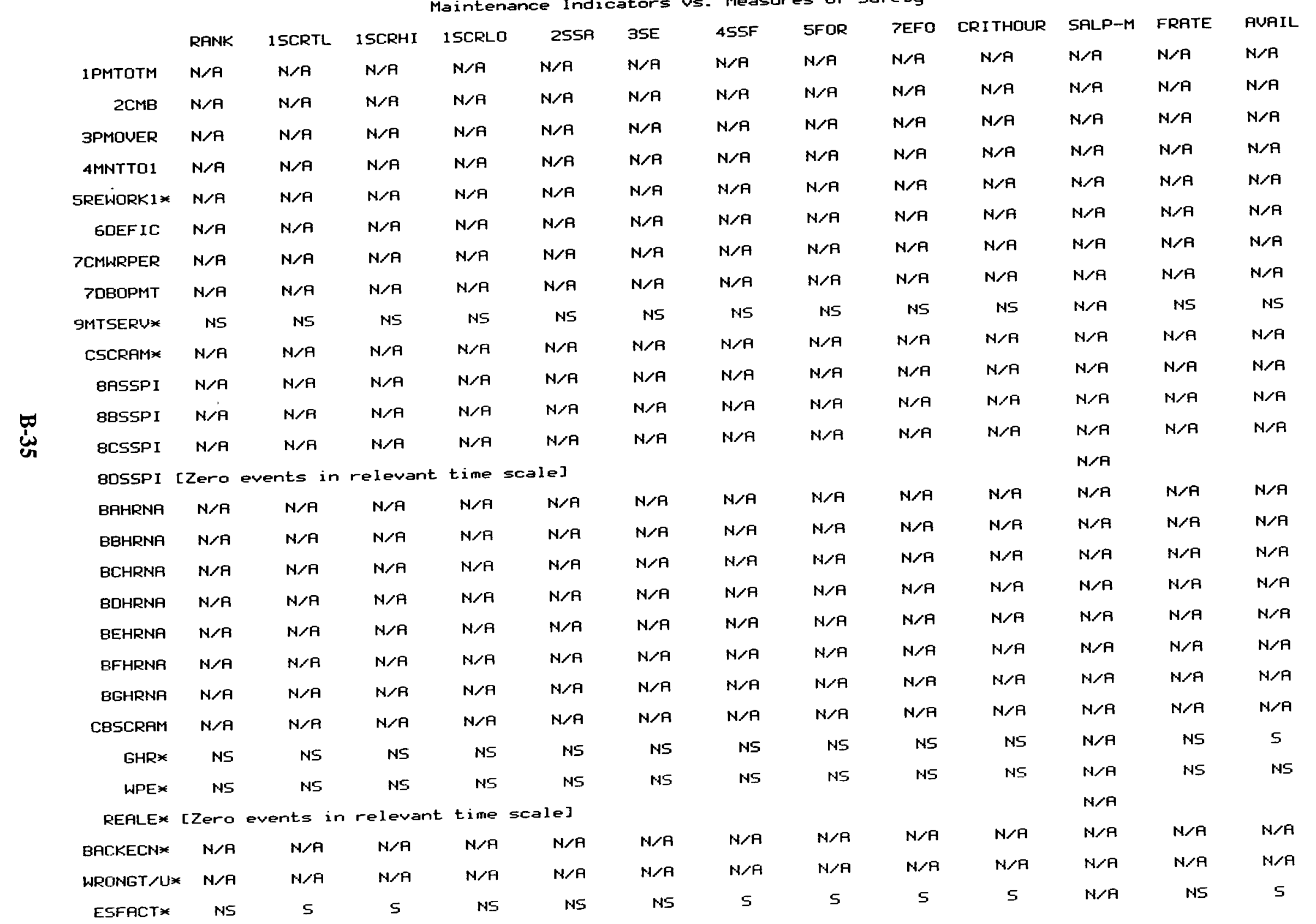


Significant Maintenance Indicator/Measure of Safety Relationships

\begin{tabular}{|c|c|c|c|c|}
\hline & $\begin{array}{l}\text { Indicator } \\
========= \\
\text { ESFRCT }\end{array}$ & $\begin{array}{l}\text { Measure of Safety } \\
================ \\
\text { RUAIL }\end{array}$ & $\begin{array}{c}\text { Time } \operatorname{Shift} \\
======== \\
0\end{array}$ & $\begin{array}{c}\text { Conf i dence } \\
======== \\
80 \%\end{array}$ \\
\hline & ESFACT & CR I THOUR & 0 & $85 \%$ \\
\hline & ESFRCT & EFO & 0 & $90 \%$ \\
\hline & ESFACT & SSF & D & $82 \%$ \\
\hline & ESFACT & SCRHI & 0 & $96 \%$ \\
\hline & ESFACT & SCRTL & D & $95 \%$ \\
\hline & ESFACT & FOR & +1 & $80 \%$ \\
\hline & GHR & RUAIL & 0 & $99 \%$ \\
\hline
\end{tabular}





B-37 

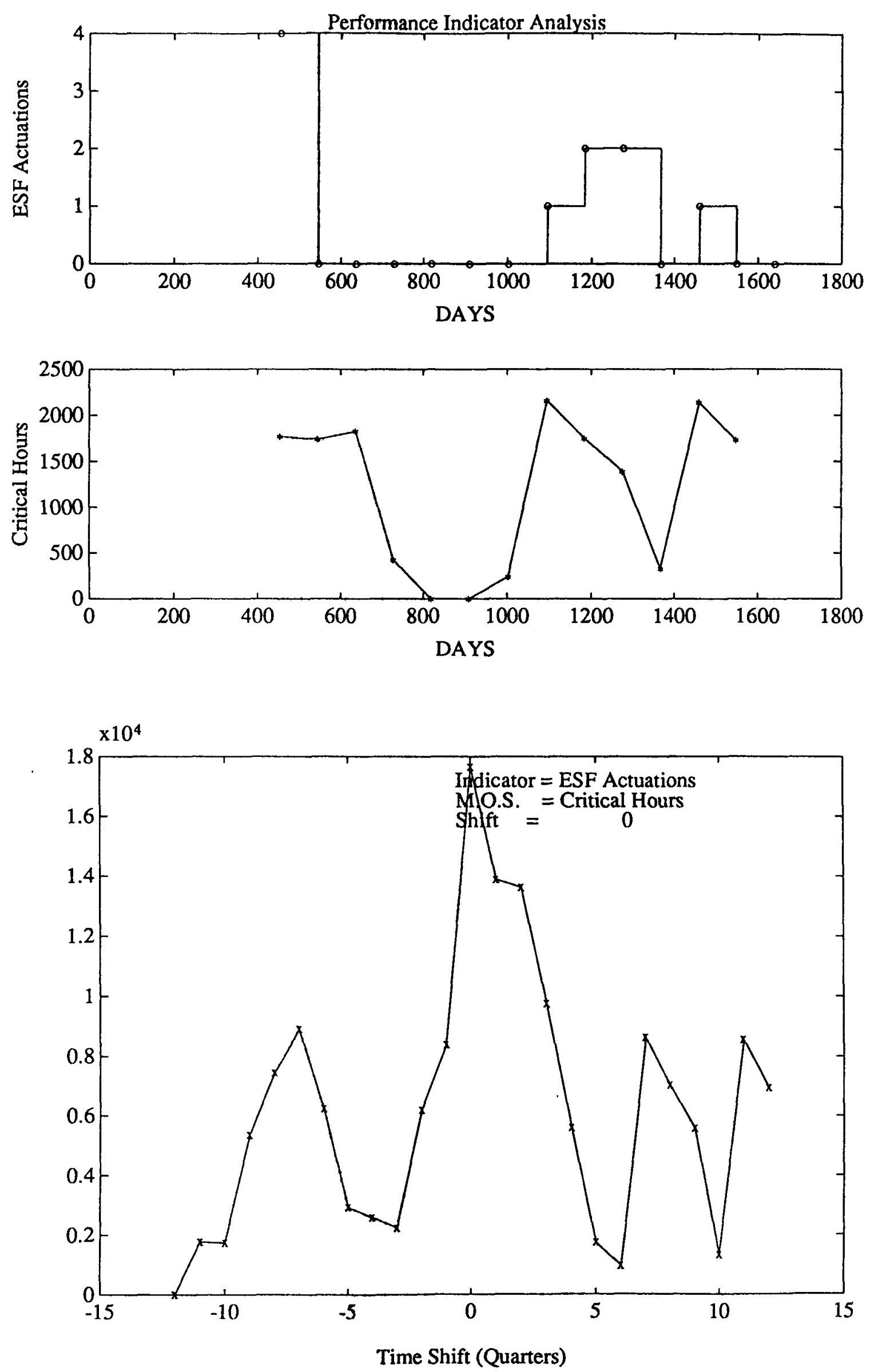

\section{B-38}



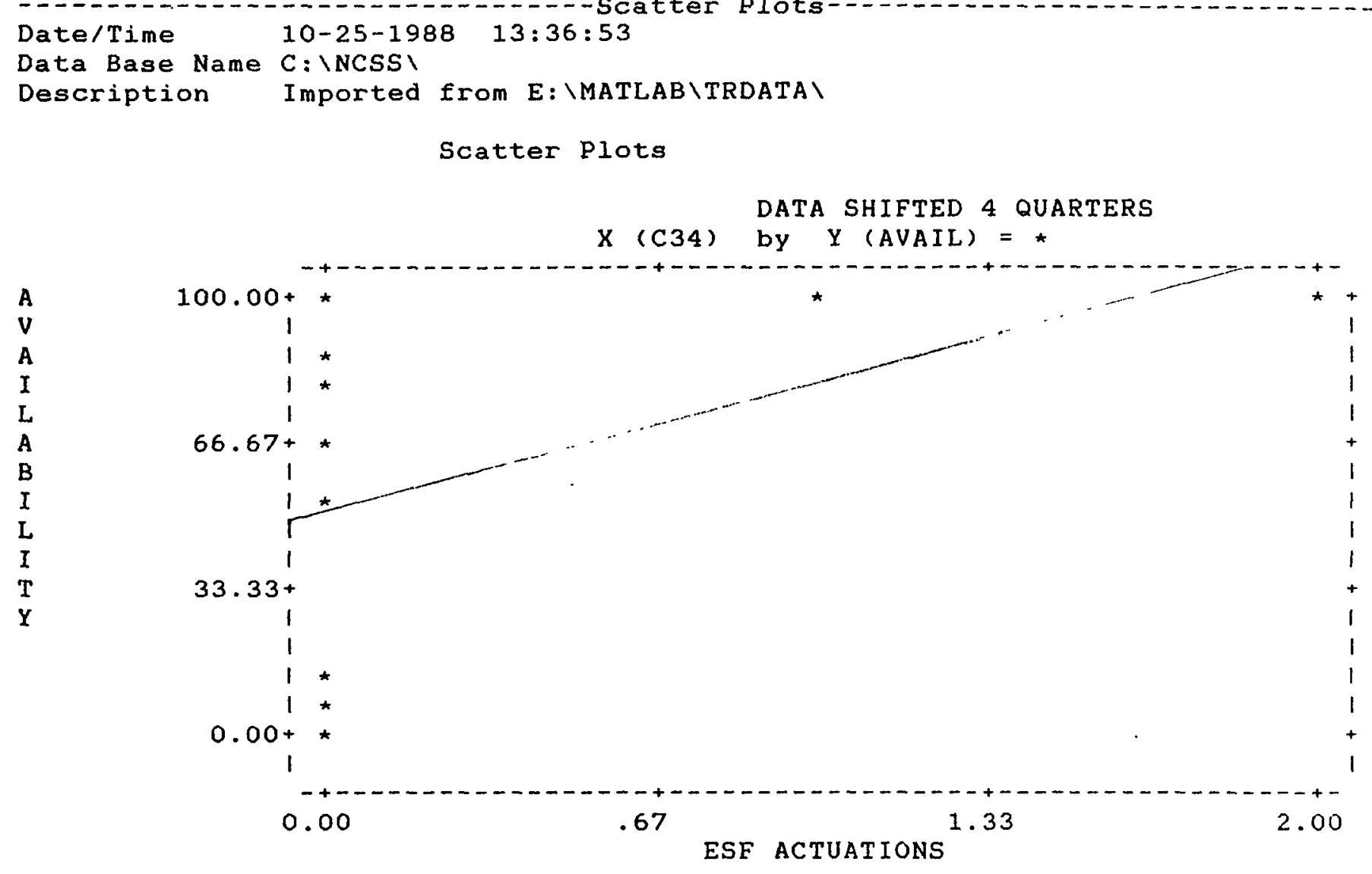

... W Accounting Report

$\begin{array}{lllrlll}\text { Number of points below minimum } & (X): & 0 & (Y): & 0 \\ \text { Number of points above maximum } & (X): & 0 & (Y): & 0 \\ \text { Number of points with missing values } & (X): & 4 & (Y): & 0 \\ \text { Number of points plotted } & & : & 10 & & & \end{array}$



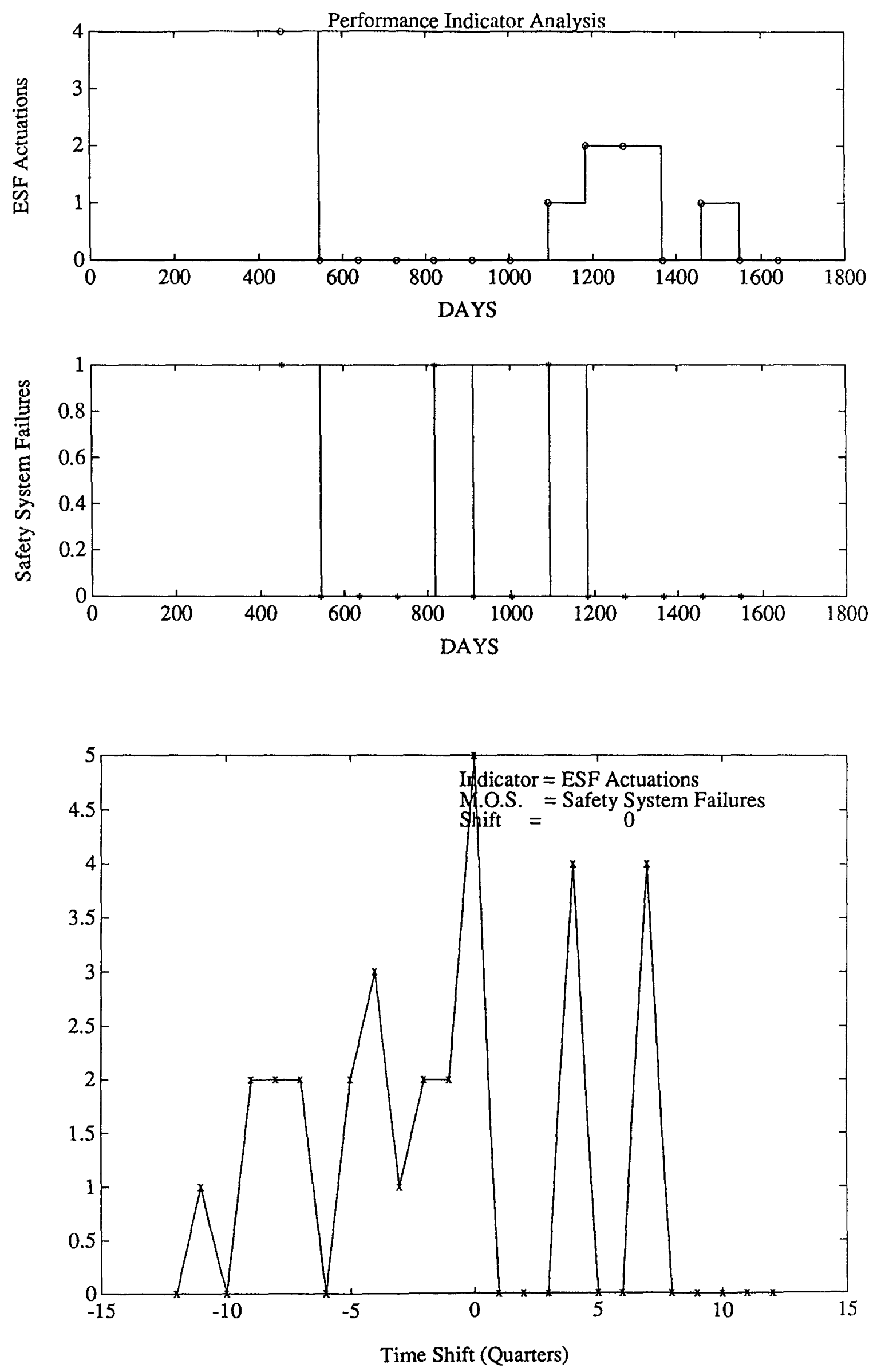

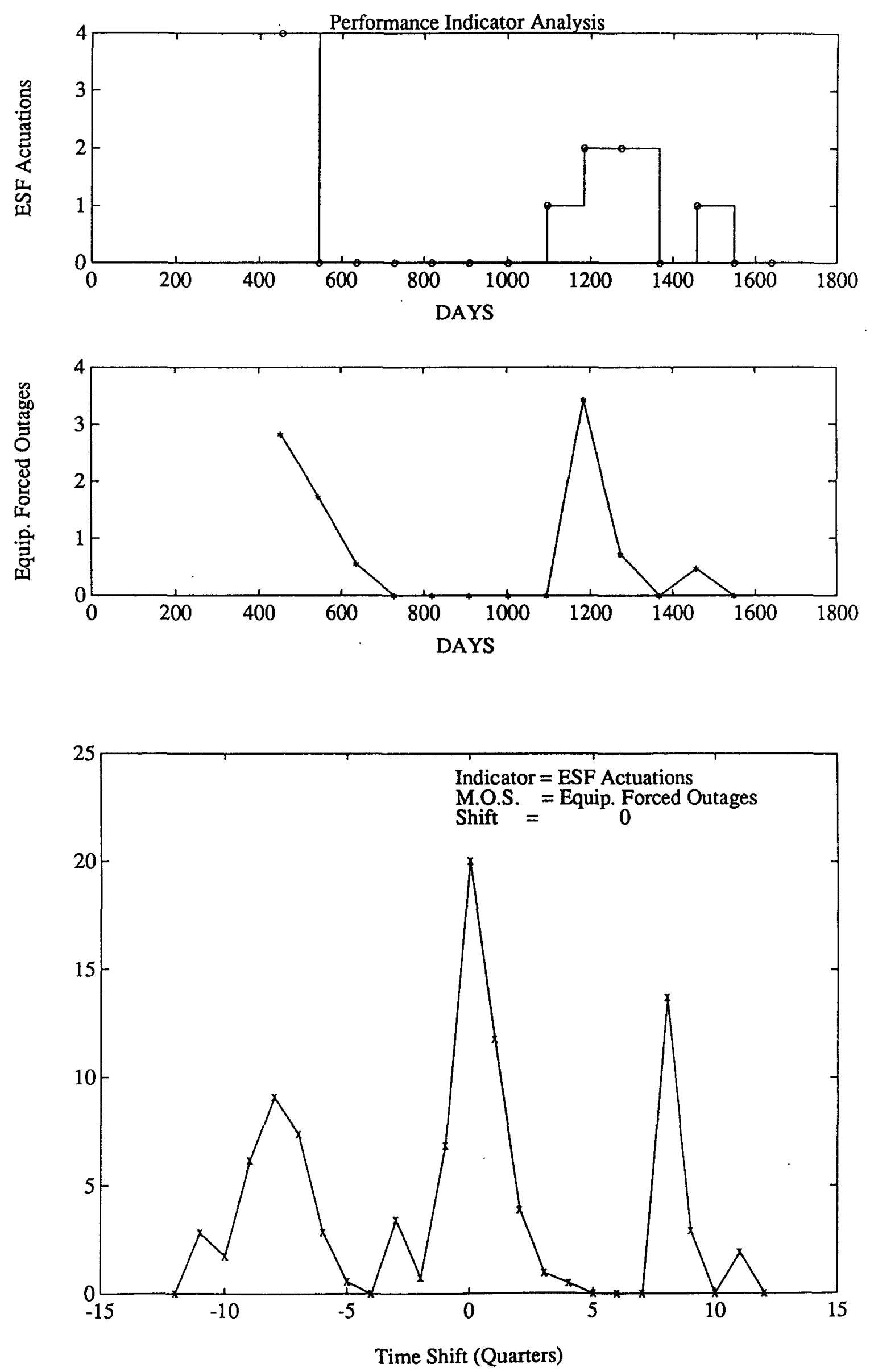

B-41 

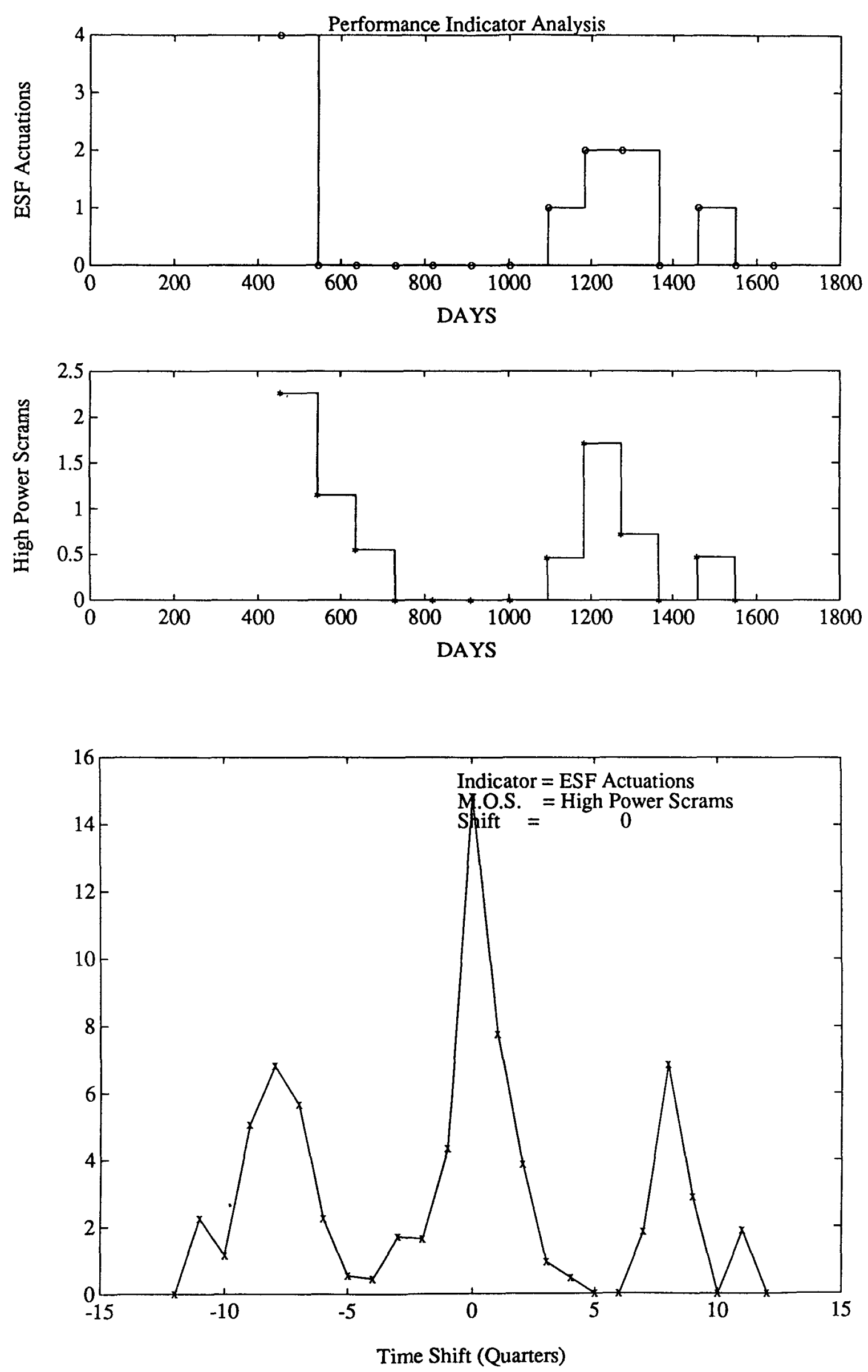

B-42 

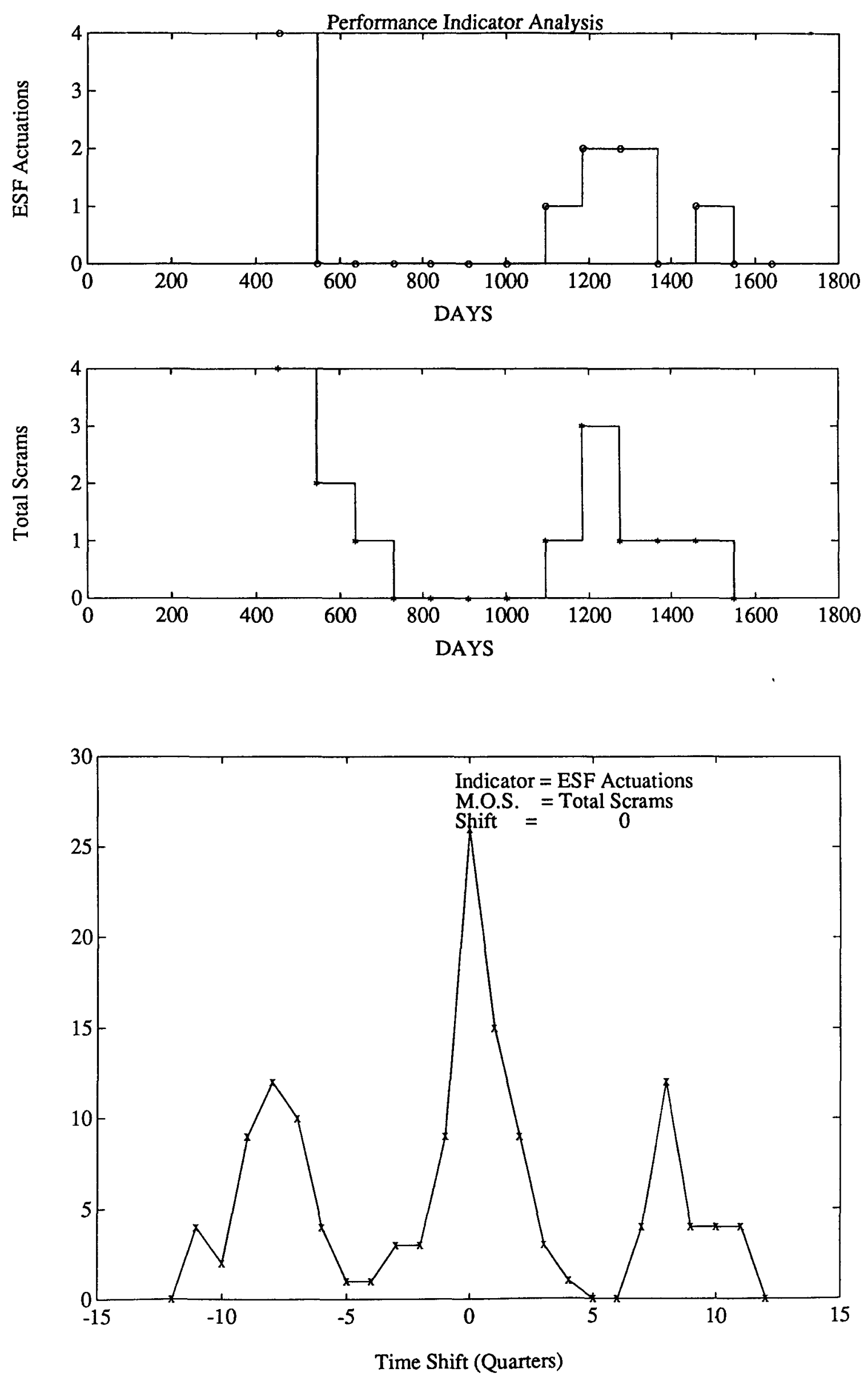

B-43 

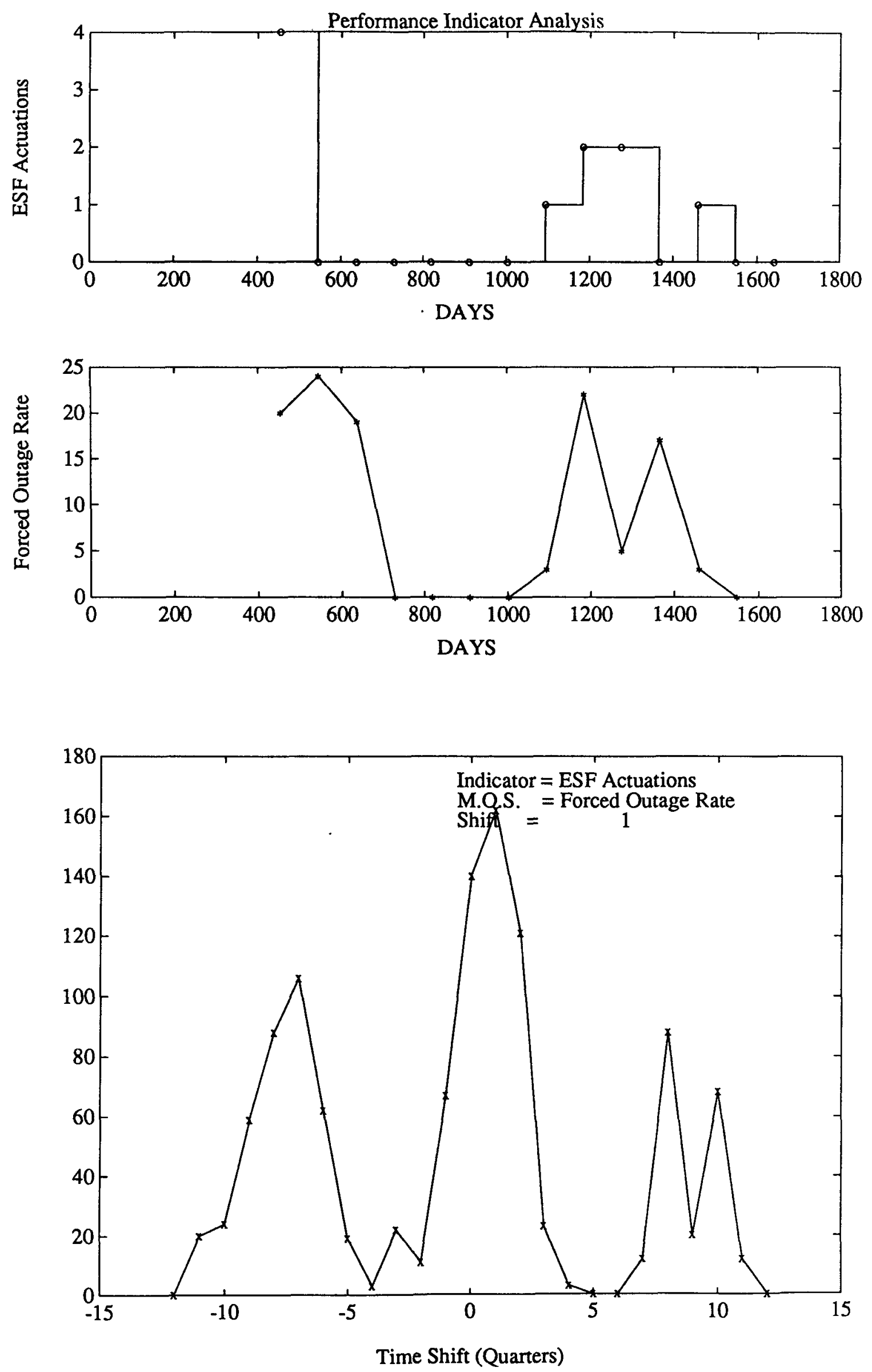

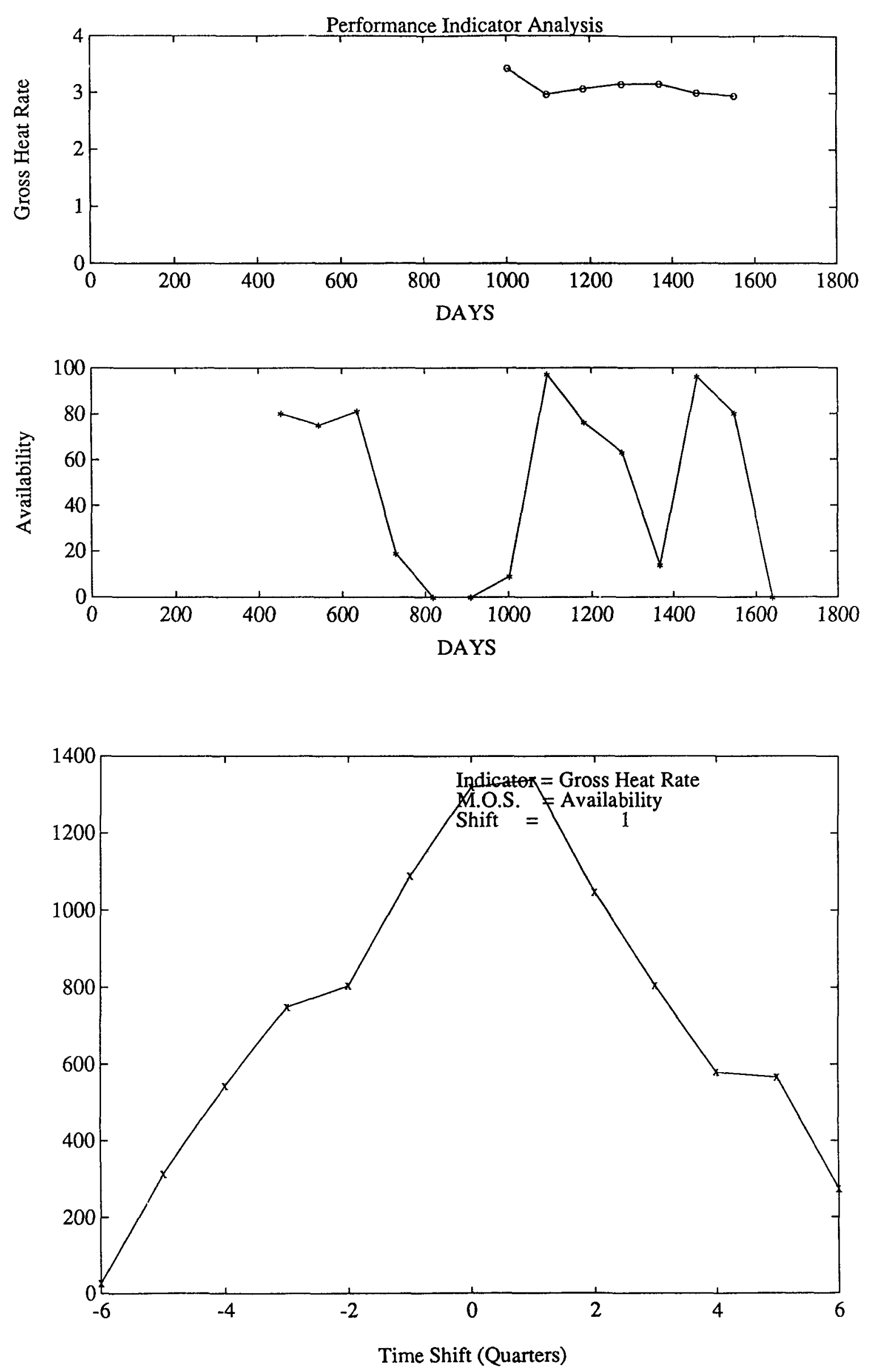

B-45 

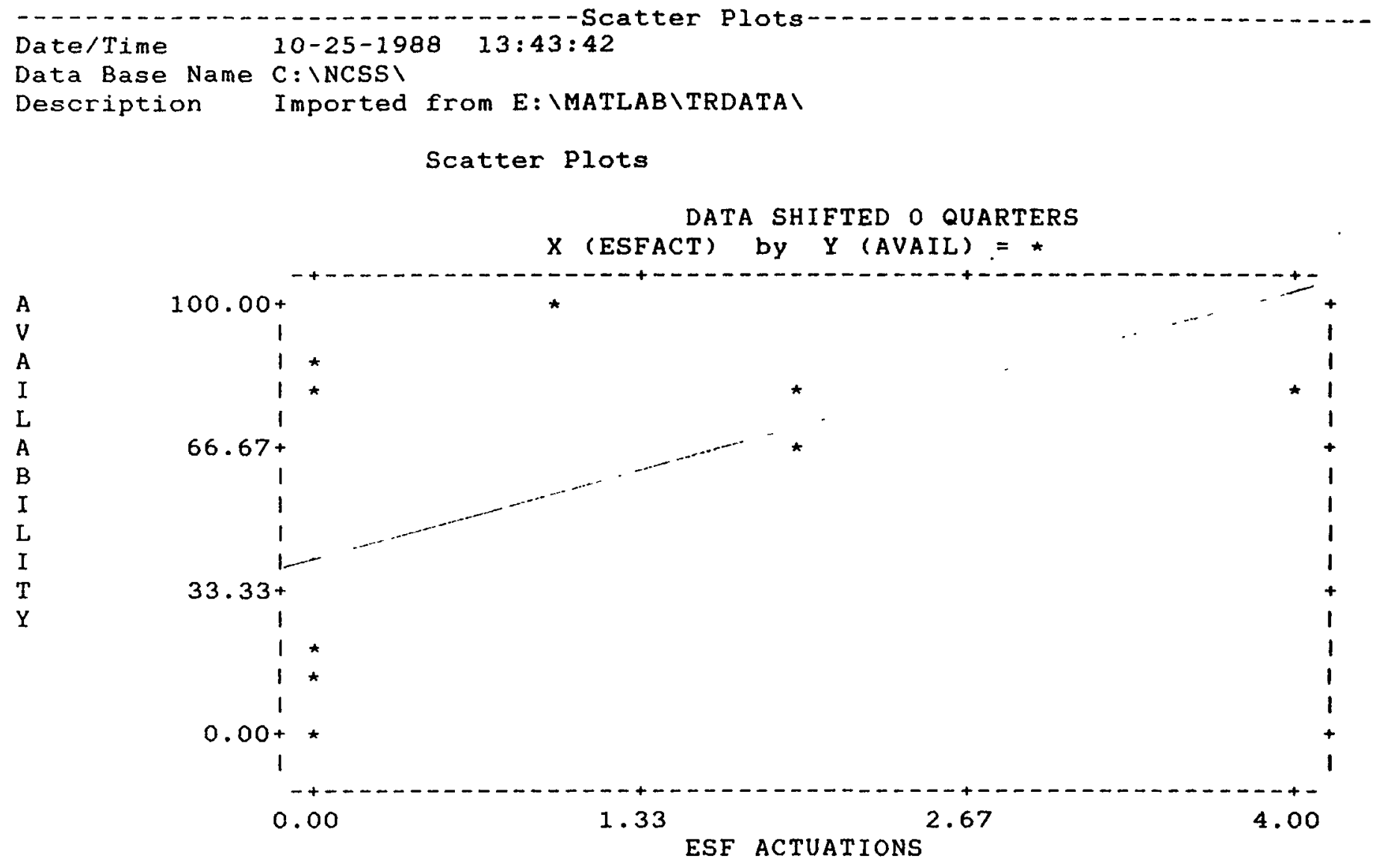

1.w Accounting Report

$\begin{array}{llrrr}\text { Number of points below minimum } & (X): & 0 & (Y): & 0 \\ \text { Number of points above maximum } & (X): & 0 & (Y): & 0 \\ \text { Number of points with missing values } & (X): & 0 & (Y): & 0 \\ \text { Number of points plotted } & & : & 14 & \end{array}$




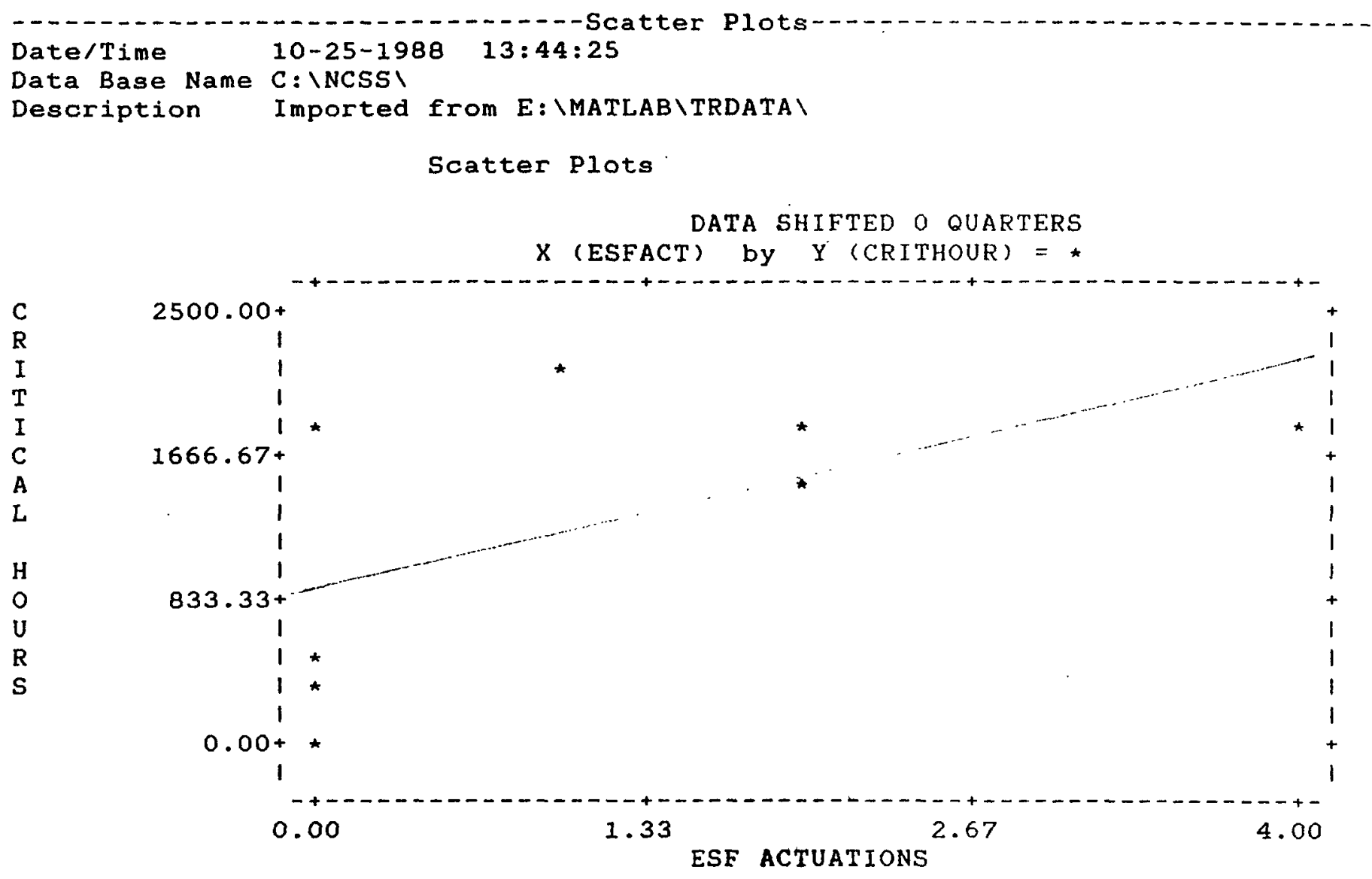

h... Accounting Report

$\begin{array}{lllllll}\text { Number of points below minimum } & (X): & 0 & (Y): & 0 \\ \text { Number of points above maximum } & (X): & 0 & (Y): & 0 \\ \text { ivuluber of points with missing values } & (X): & 0 & (Y): & 1 \\ \text { Number of points plotted } & & : & 13 & & & \end{array}$




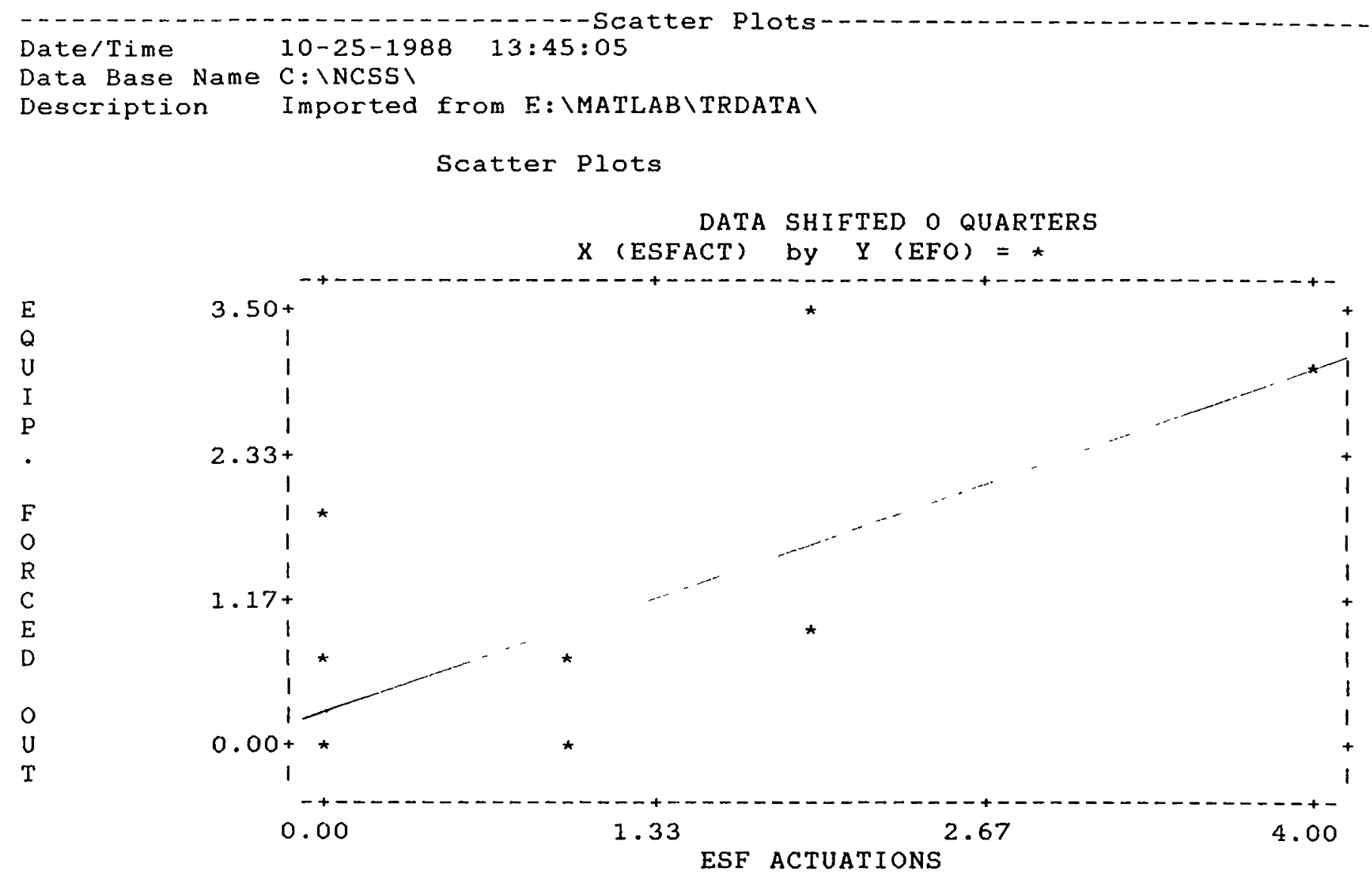

1. W Accounting Report

Number of points below minimum Number of points above maximum Number of points with missing values Number of points plotted

\begin{tabular}{|c|c|c|c|c|}
\hline$(X)$ & : & 0 & $(Y)$ & : \\
\hline$(X)$ & : & 0 & $(Y)$ & : \\
\hline$(X)$ & : & 0 & $(Y)$ & : \\
\hline & : & 13 & & \\
\hline
\end{tabular}




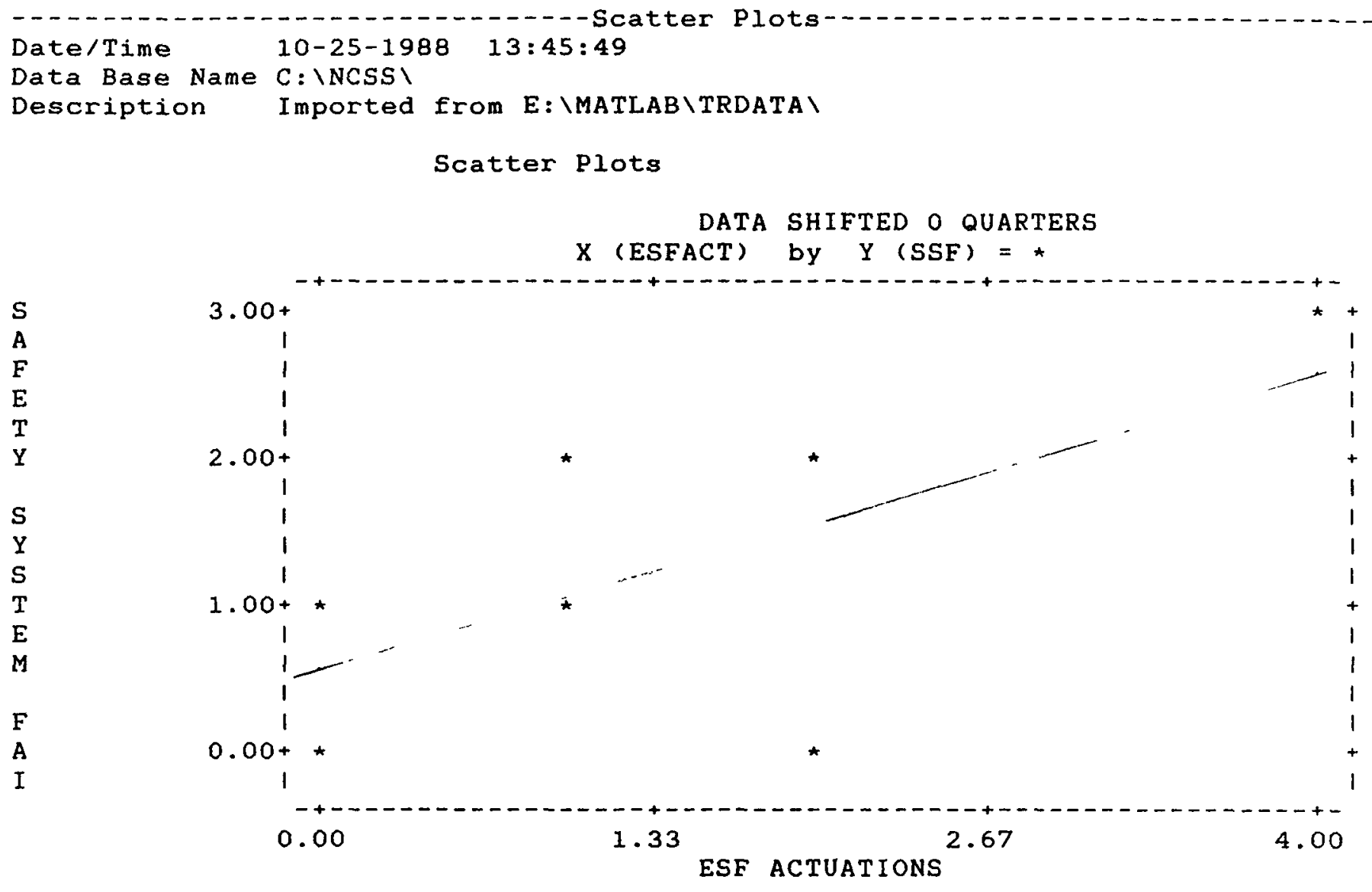

1. N Accounting Report

$\begin{array}{lllrl:l}\text { Number of points below minimum } & (X): & 0 & (Y): & 0 \\ \text { Number of points above maximum } & (X): & 0 & (Y): & 0 \\ \text { Number of points with missing values }(X): & 0 & (Y): & 1 \\ \text { Number of points plotted } & & : & 13 & & \end{array}$




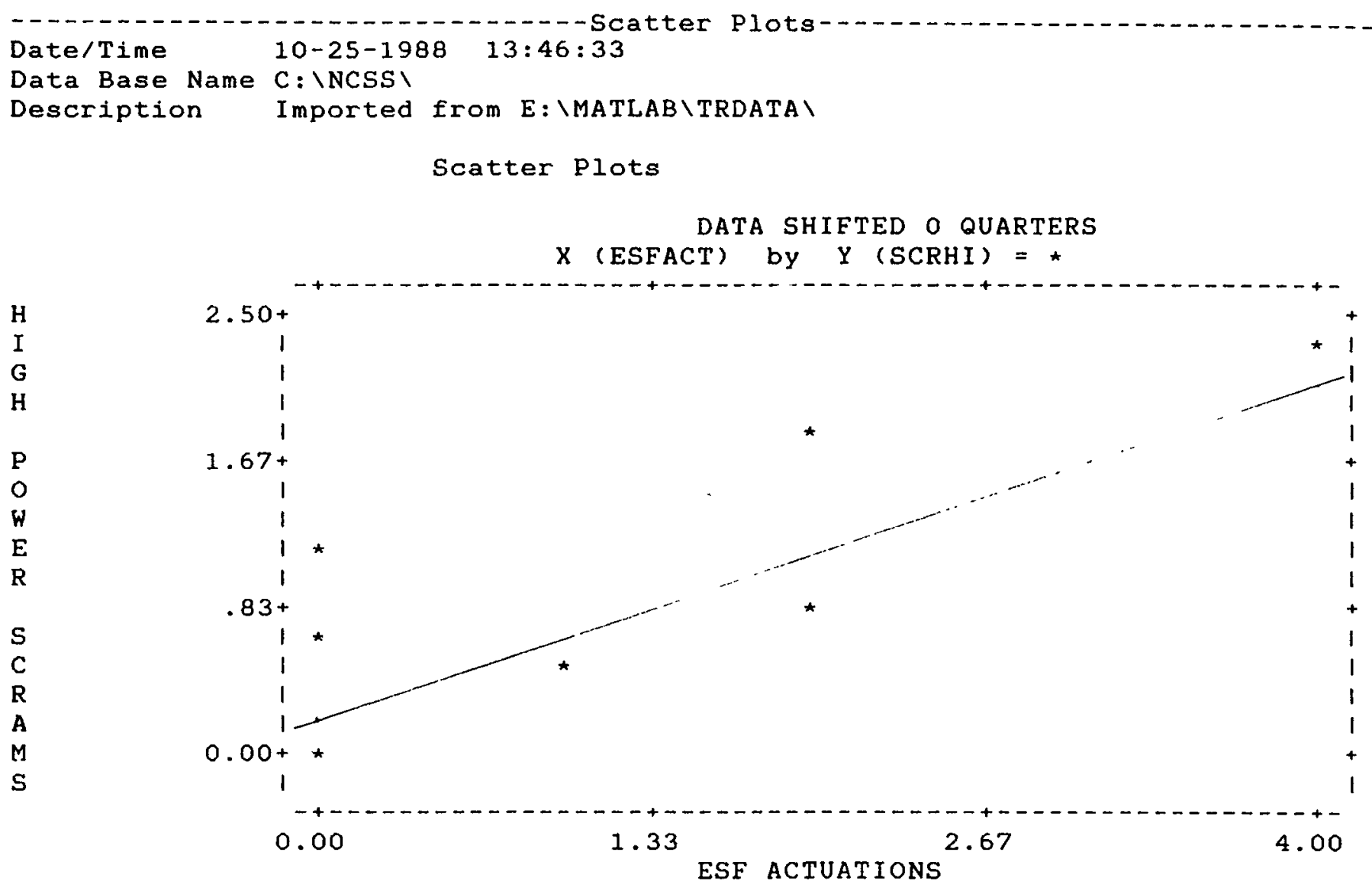

W-w Accounting Report

$\begin{array}{llrrr}\text { Number of points below minimum } & (X): & 0 & (Y): & 0 \\ \text { Number of points above maximum } & (X): & 0 & (Y): & 0 \\ \text { Number of points with missing values } & (X): & 0 & (Y): & 1 \\ \text { Number of points plotted } & & : & 13 & \end{array}$




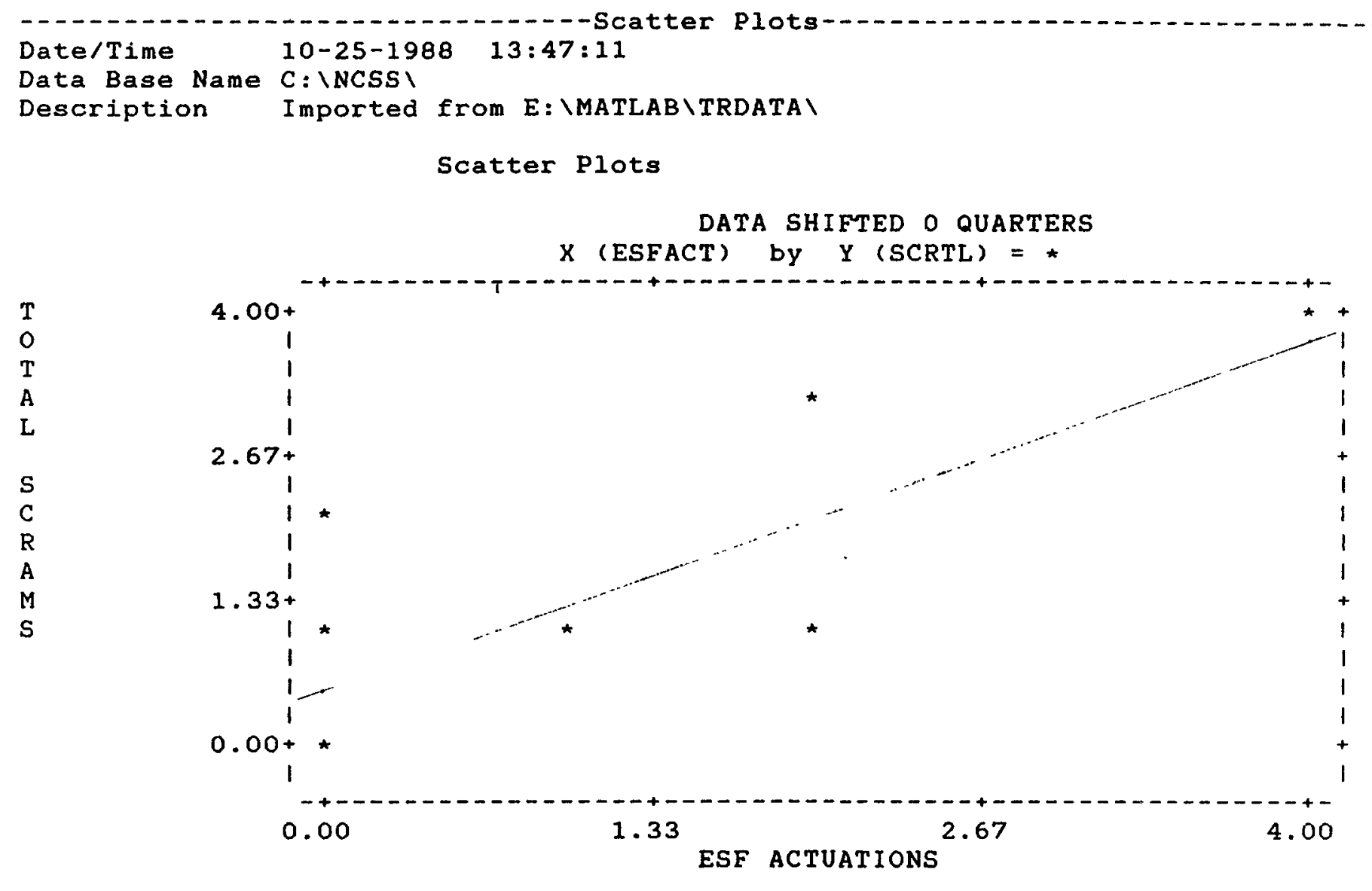

1.uw Accounting Report

$\begin{array}{lllllll}\text { Number of points below minimum } & (X): & 0 & (Y): & 0 \\ \text { Number of points above maximum } & (X): & 0 & (Y): & 0 \\ \text { Number of points with missing values }(X): & 0 & (Y): & 1 \\ \text { Number of points plotted } & & : & 13 & & \end{array}$




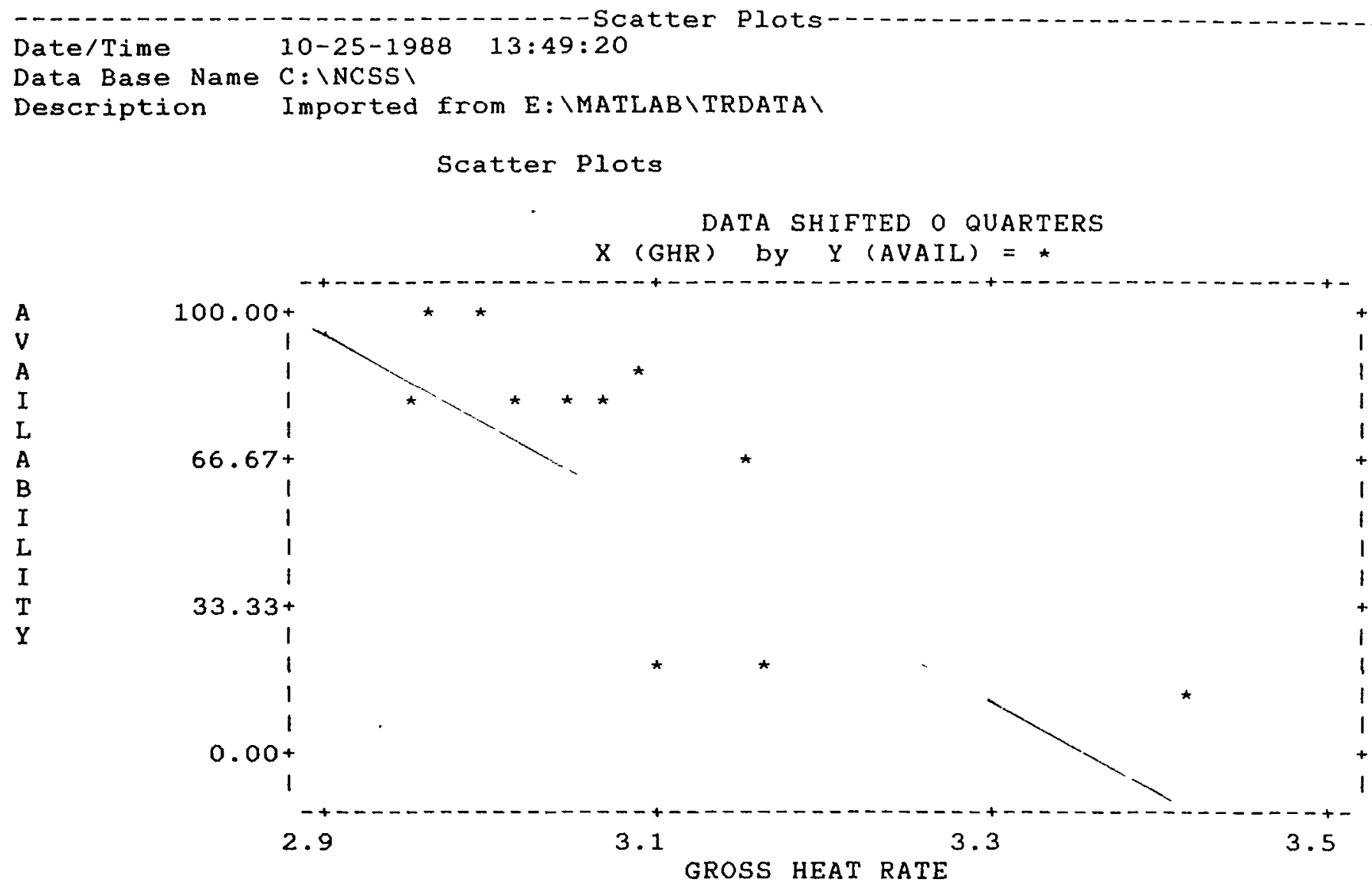

\section{* Accounting Report}

$\begin{array}{lllllll}\text { Number of points below minimum } & (X): & 0 & (Y): & 0 \\ \text { Number of points above maximum } & (X): & 0 & (Y): & 0 \\ \text { Number of points with missing values }(X): & 2 & (Y): & 0 \\ \text { Number of points plotted } & & : & 11 & & & \end{array}$




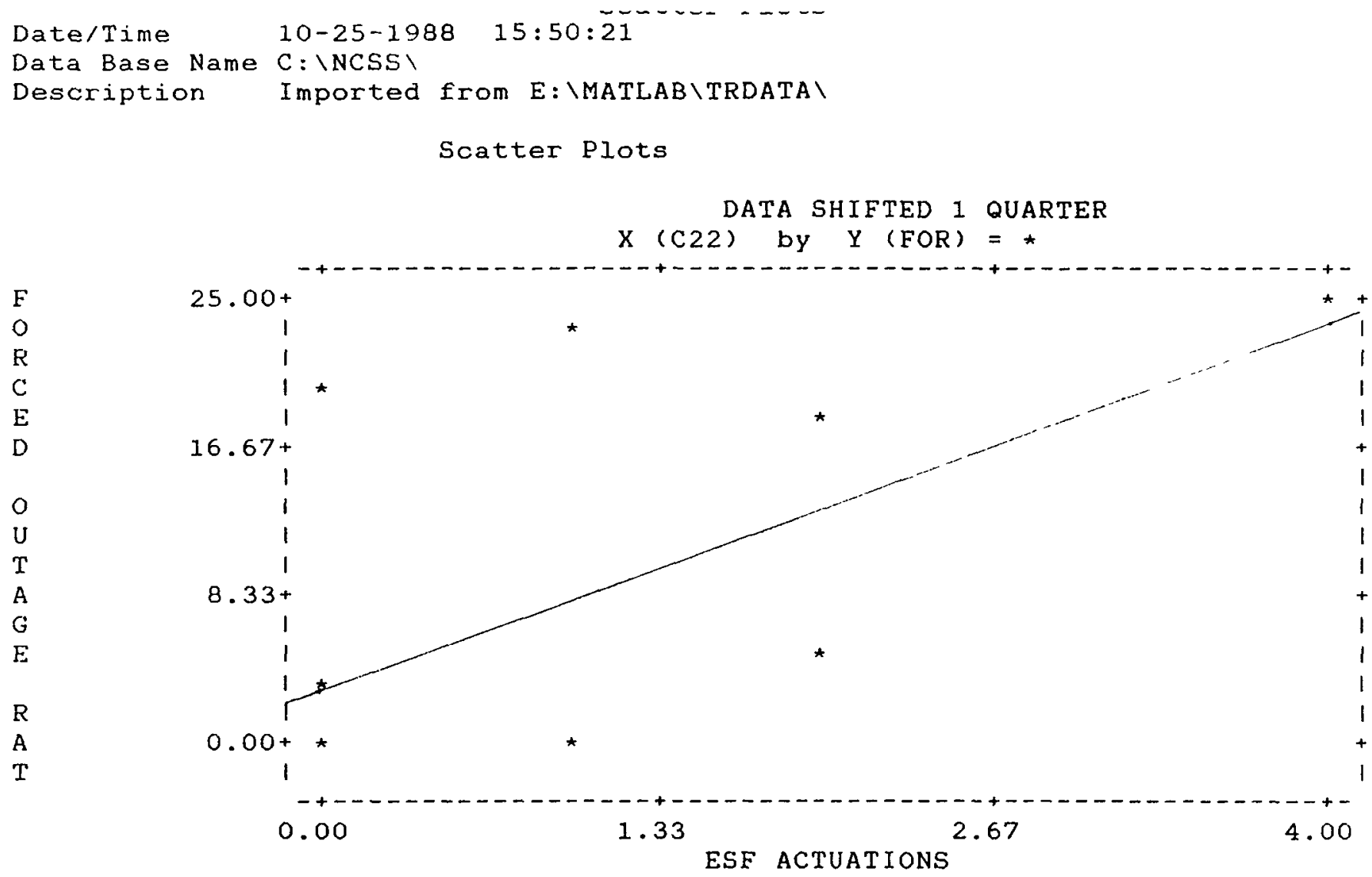

Row Accounting Report

$\begin{array}{lllllll}\text { lber of points below minimum } & (X): & 0 & (Y): & 0 \\ \text { umber of points above maximum } & (X): & 0 & (Y): & 0 \\ \text { umber of points with missing values }(X): & 1 & (Y): & 1 \\ \text { umber of points plotted } & & : & 12 & & & \end{array}$




\section{Detailed Results for Plant F}


Maintenance Indicators vs. Measures of Safety




Significant Maintenance Indicator/Measure of Safety Relationships

\begin{tabular}{|c|c|c|c|}
\hline $\begin{array}{l}\text { Indicator } \\
========\end{array}$ & $\begin{array}{l}\text { Measure of Safety } \\
===============\end{array}$ & $\begin{array}{l}\text { Time Shift } \\
========\end{array}$ & $\begin{array}{l}\text { Conf i dence } \\
========\end{array}$ \\
\hline ESFACT & SCRHI & +7 & $87 \%$ \\
\hline ESFACT & EFO & +7 & $87 \%$ \\
\hline MTSERV & SALP & 0 & $92 \%$ \\
\hline GHR & AUAIL & 0 & $96 \%$ \\
\hline GHR & CR I THOUR & 0 & $97 \%$ \\
\hline
\end{tabular}



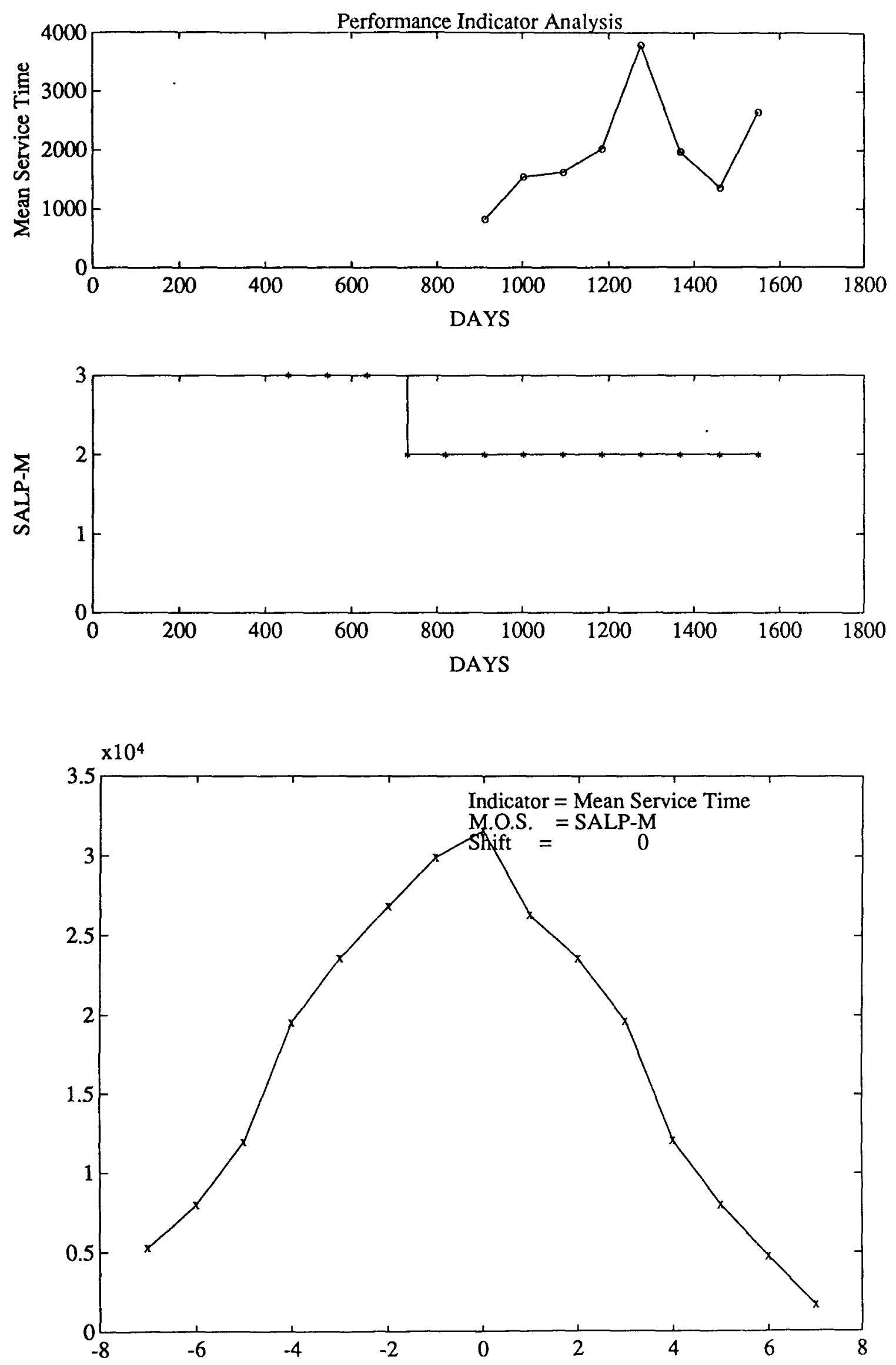

Time Shift (Quarters) 

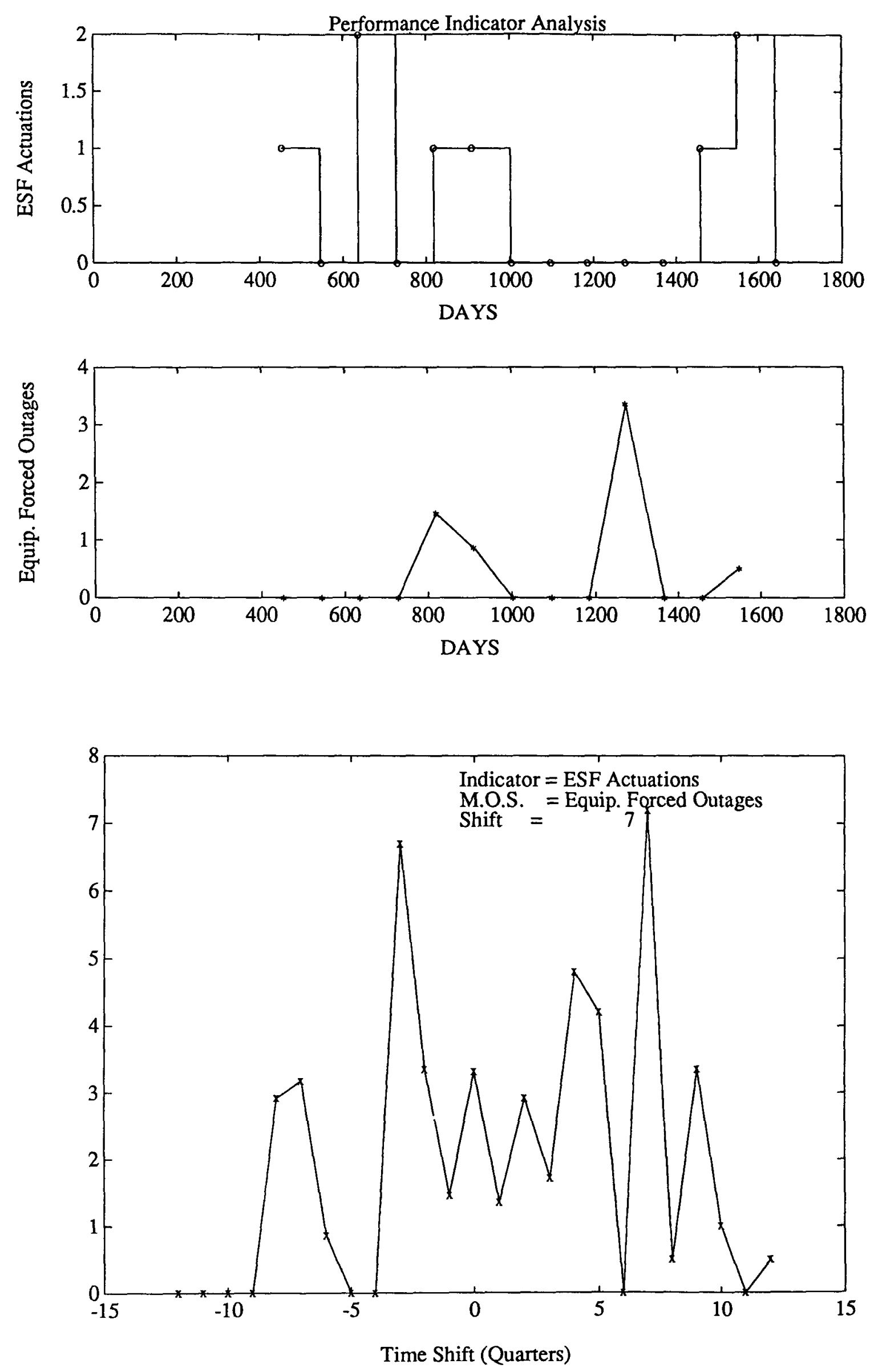

B-58 

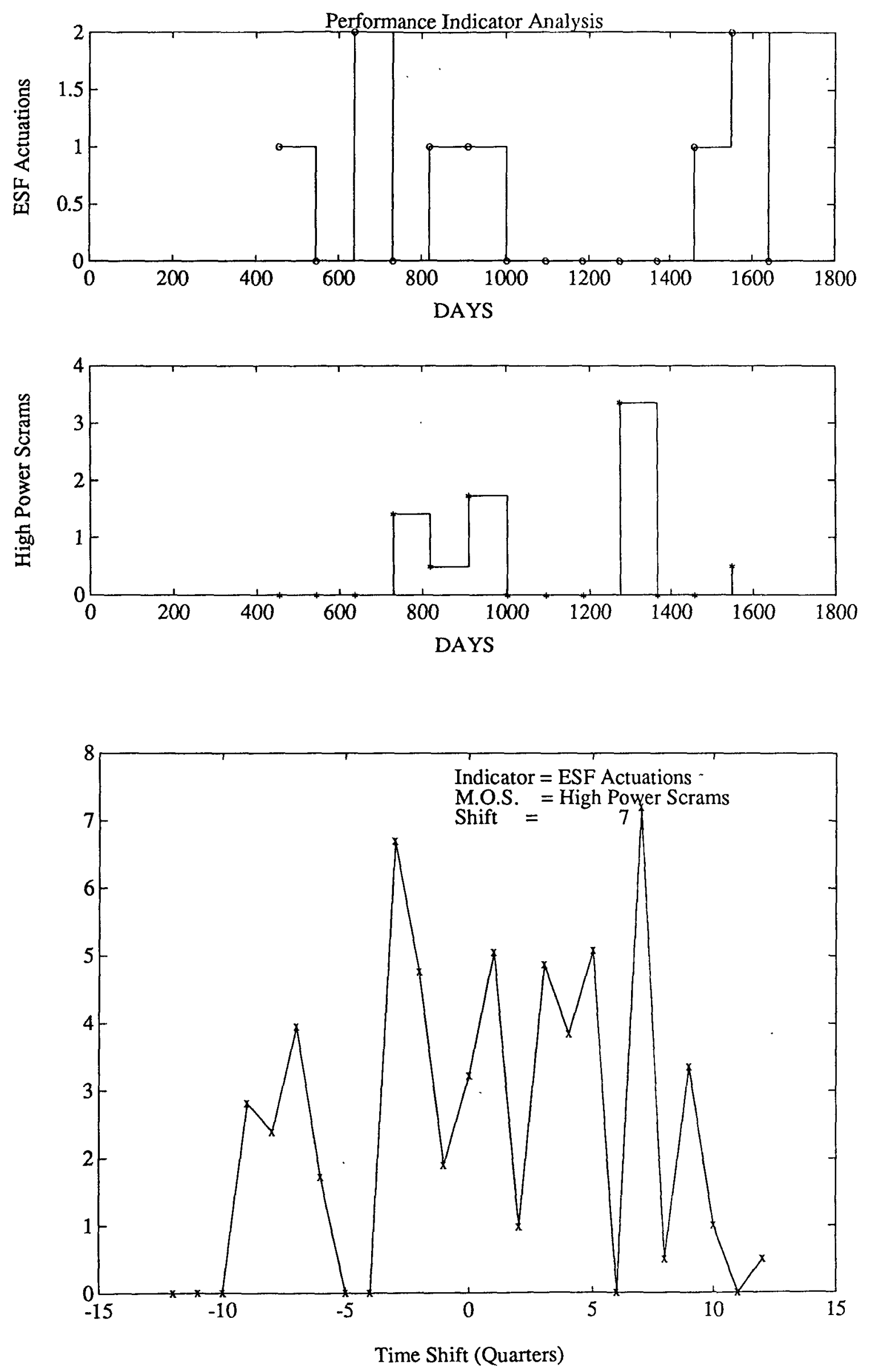

B-59 

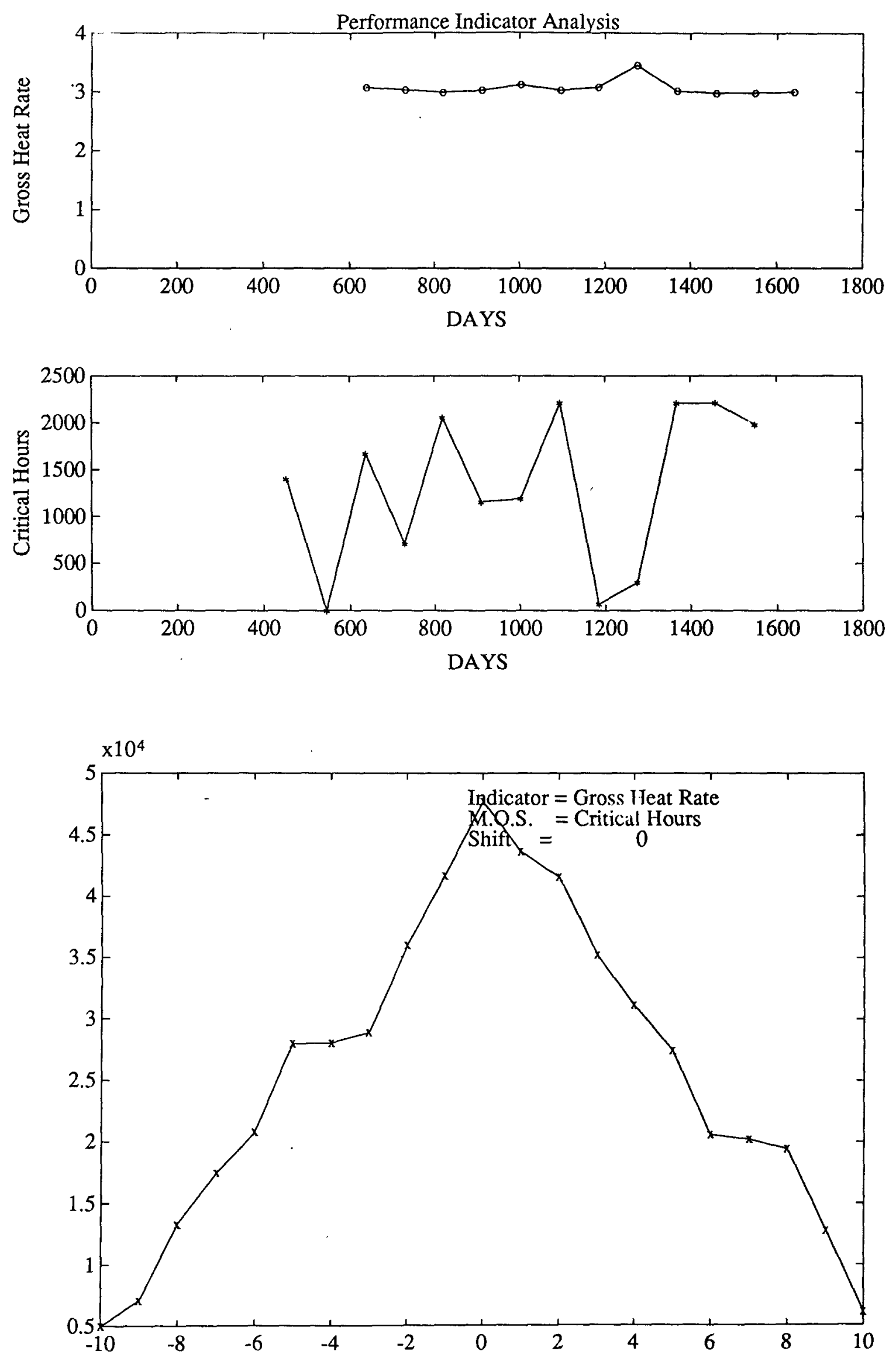

Time Shift (Quarters)

\section{B-60}



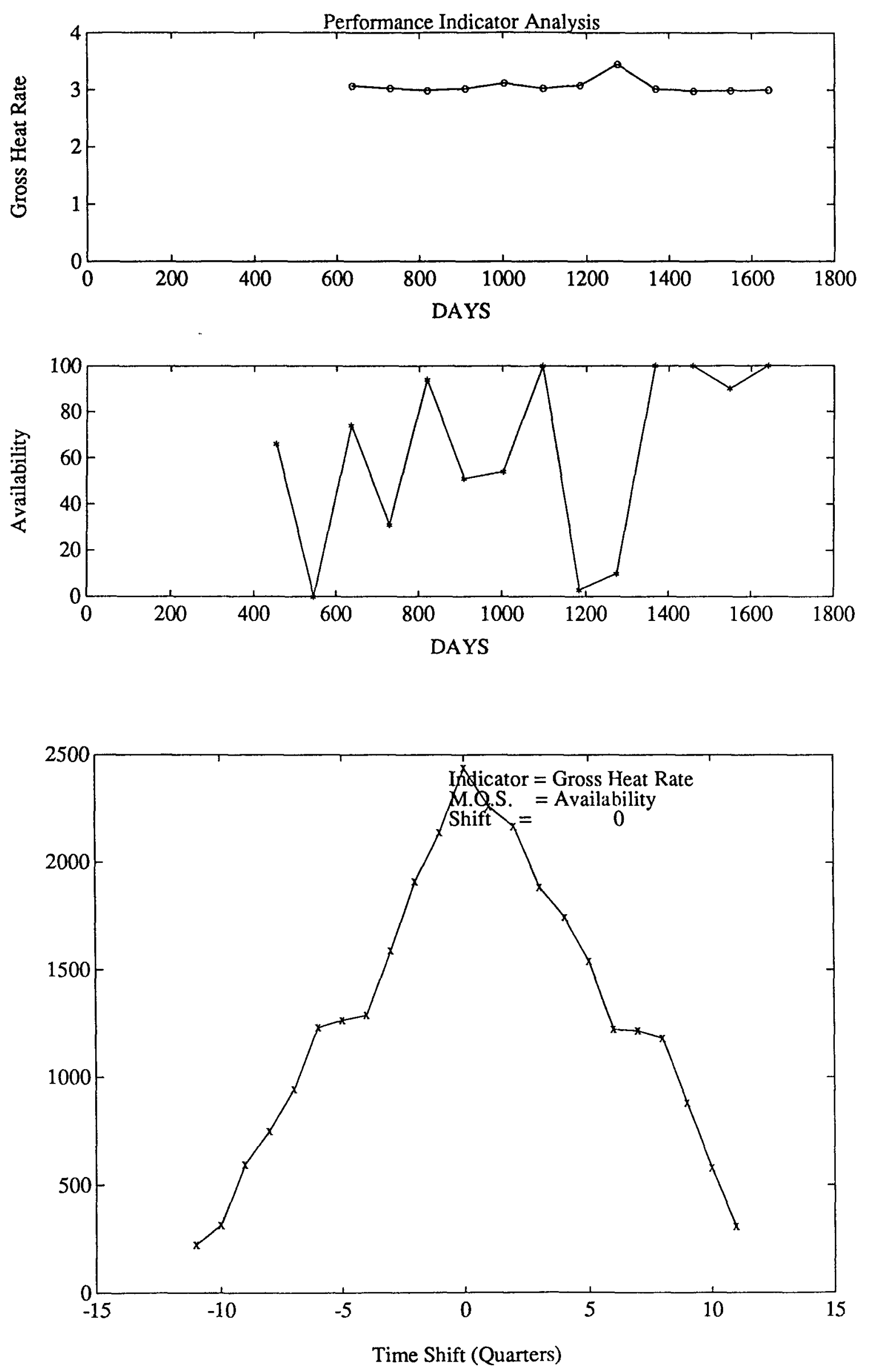

B-61 




I Accounting Report

$\begin{array}{lllllll}\text { Number of points below minimum } & (X): & 0 & (Y): & 0 \\ \text { Number of points above maximum } & (X): & 0 & (Y): & 0 \\ \text { Number of points with missing values }(X): & 5 & (Y): & 0 \\ \text { Number of points plotted } & & : & 8 & & \end{array}$




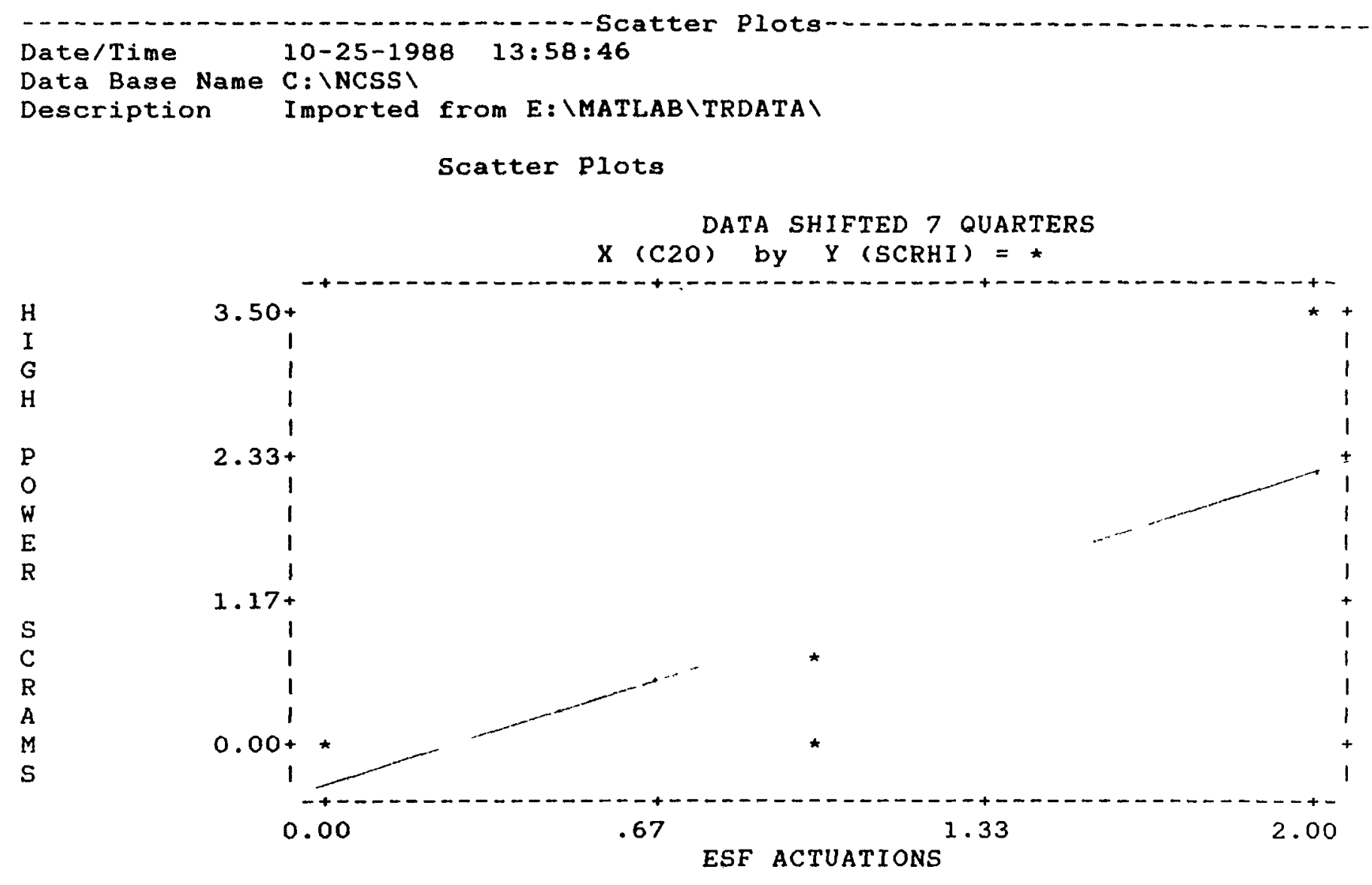

1. -w Accounting Report

$\begin{array}{lllllll}\text { Number of points below minimum } & (X): & 0 & (Y): & 0 \\ \text { Number of points above maximum } & (X): & 0 & (Y): & 0 \\ \text { Number of points with missing values }(X): & 7 & (Y): & 1 \\ \text { Number of points plotted } & & : & 6 & & \end{array}$




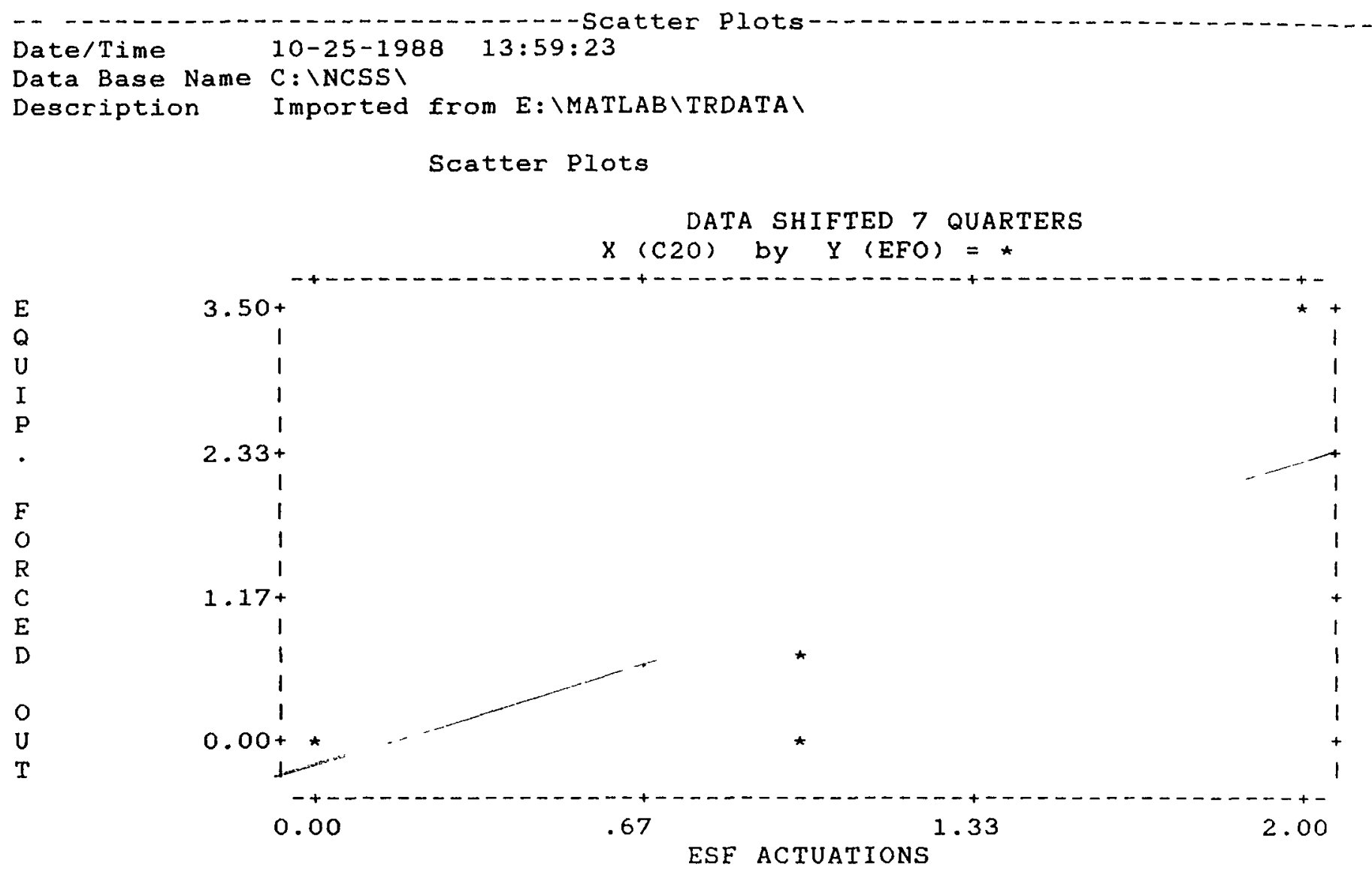

w-w Accounting Report

$\begin{array}{lllllll}\text { Number of points below minimum } & (X): & 0 & (Y): & 0 \\ \text { Number of points above maximum } & (X): & 0 & (Y): & 0 \\ \text { Number of points with missing values }(X): & 7 & (Y): & 1 \\ \text { Number of points plotted } & & : & 6 & & & \end{array}$




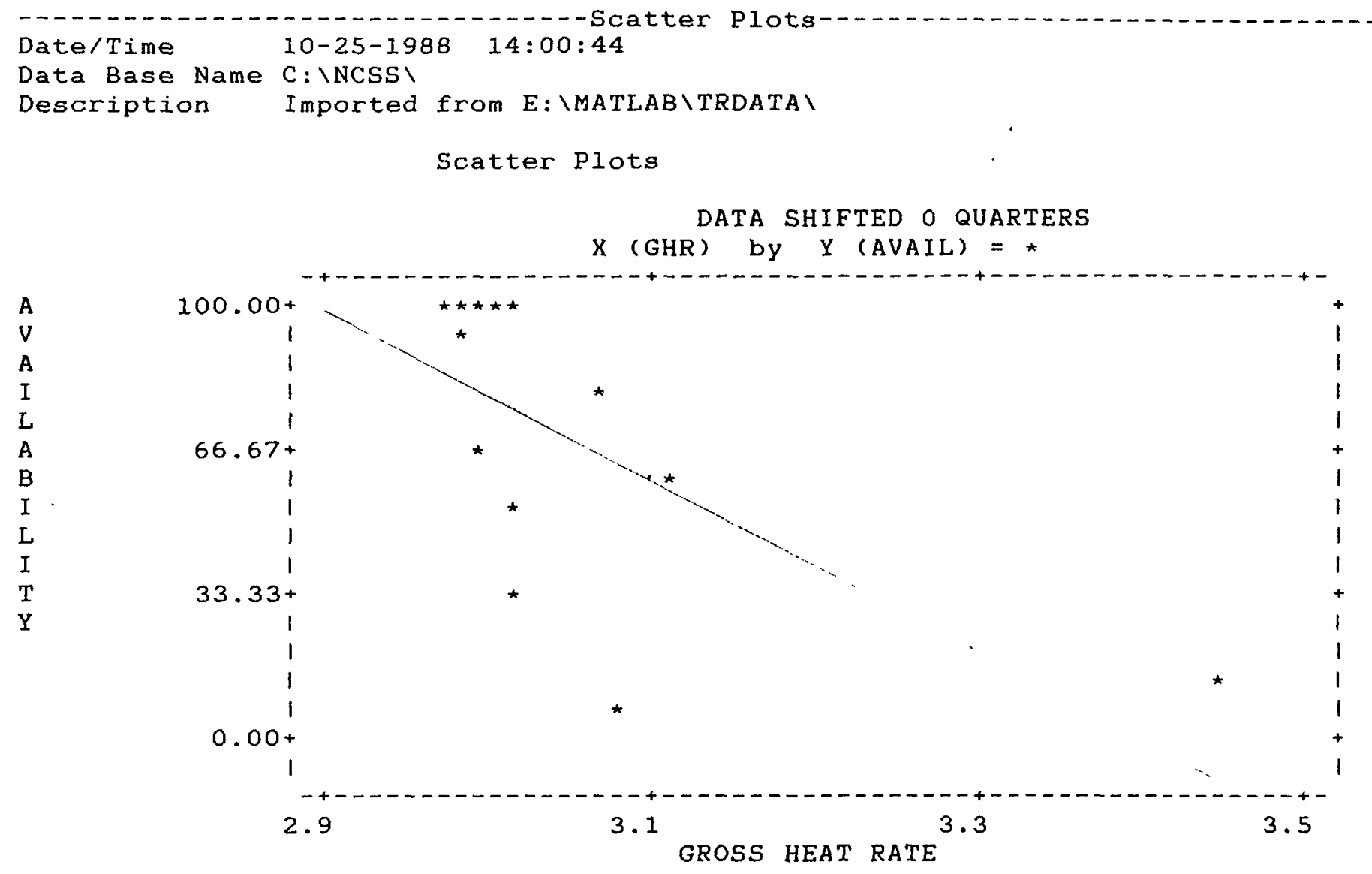

* Accounting Report

of points above maximum

$(X): 0 \quad(Y): 0$

Number of points with missing values

Number of points plotted

$\begin{array}{rrrrr}(X): & 0 & (Y): & 0 \\ (X): & 1 & (Y): & 0 \\ & : & 13 & & \end{array}$




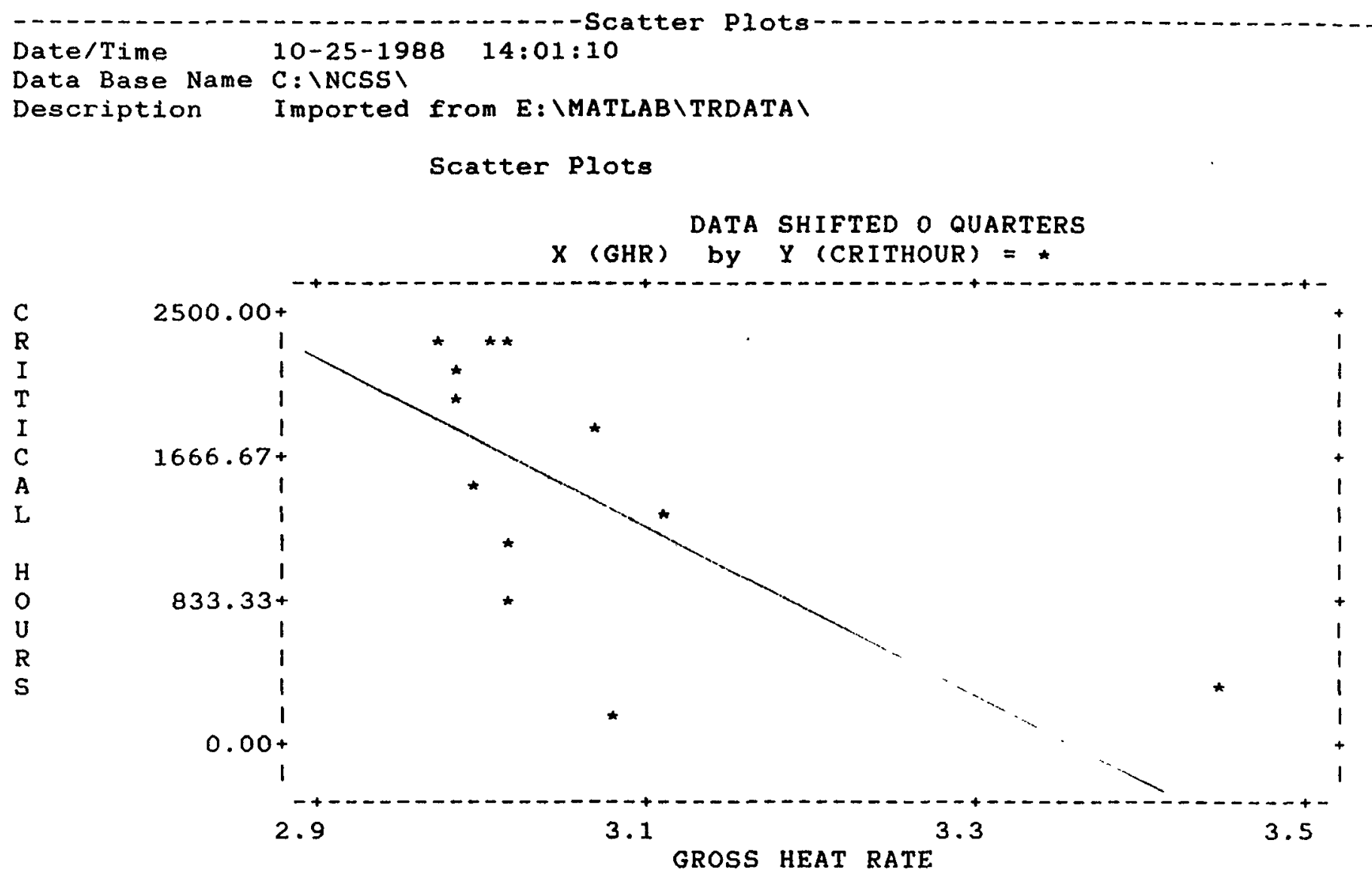

k-w Accounting Report

Number of points below minimum

Number of points above maximum

Number of points with missing values

Number of points plotted

\begin{tabular}{|c|c|c|c|c|}
\hline$(X)$ & : & 0 & $(Y)$ & : \\
\hline$(X)$ & : & 0 & $(Y)$ & : \\
\hline (X) & : & 1 & $(Y)$ & : \\
\hline & : & 12 & & \\
\hline
\end{tabular}


Detailed Results for Plant G 
Maintenance Indicators vs. Measures of Safety

\begin{tabular}{|c|c|c|c|c|c|c|c|c|c|c|c|c|c|}
\hline & RANK & 1SCRTL & $15 \mathrm{CRHI}$ & $15 C R L D$ & $255 A$ & 3SE & $45 S F$ & SFOR & PEFO & CR I THOUR & $S A L P-M$ & FRATE & FUAIL \\
\hline 1PMTOTM & $N / A$ & $N / A$ & $N / A$ & $N / A$ & $N / A$ & $N / P$ & $N / A$ & $M / A$ & $N / R$ & $N / A$ & $N / A$ & $N / A$ & $N / A$ \\
\hline $2 \mathrm{CMB}$ & $N / A$ & $N / R$ & $N / A$ & $N / A$ & $N / A$ & $N / A$ & $N / A$ & $N / A$ & $N / A$ & $N / A$ & $N / A$ & $N / A$ & $N / A$ \\
\hline 3PMOUER & $N / A$ & $N / A$ & $N / A$ & $M / A$ & $N / A$ & $\mathbf{N} / \mathrm{A}$ & $N \not A$ & $N / R$ & $N / A$ & $N / A$ & $N / A$ & $N / P$ & $N / R$ \\
\hline 4MNTTO1 & $N / A$ & $N / A$ & $M / A$ & $N / A$ & $\mathrm{~N} / \mathrm{A}$ & $N / A$ & $N A$ & $N / A$ & $N / A$ & $N / A$ & $M / A$ & $M / A$ & $N / A$ \\
\hline 5REWDRK $1 *$ & $N / A$ & $N / A$ & $N / A$ & $N / A$ & $N / A$ & $N / A$ & $N / A$ & $\mathrm{~N} / \mathrm{A}$ & $N / A$ & $N / F$ & N/A & $N / A$ & $\mathrm{~N} / \mathrm{A}$ \\
\hline GDEF IC & NS & NS & NS & $N / A$ & $N / A$ & NS & NS & NS & NS & NS & NS & NS & 5 \\
\hline 7CMWRPER & $N / A$ & $W / A$ & N/A & $N / A$ & $N / A$ & $N / A$ & $M / A$ & $N / A$ & $N / A$ & N/A & $M / A$ & $N / A$ & $N / A$ \\
\hline 7OBOPMT & NS & NS & NS & N/A & $N / A$ & NS & NS & NS & NS & NS & MS & 5 & NS \\
\hline 9MTSERUX & NS & NS & NS & $N / A$ & $N / A$ & NS & NS & NS & NS & NS & NS & NS & NS \\
\hline CSCRAMX & NS & NS & NS & $N / A$ & $N / R$ & NS & NS & 5 & NS & NS & MS & $\mathbf{S}$ & NS \\
\hline BASSPI & $N / A$ & $N / A$ & $N / A$ & $N / P$ & $N / A$ & $N / A$ & $N / A$ & $N / A$ & $N / A$ & $N / A$ & $N / A$ & $N / A$ & $N / R$ \\
\hline BBSSPI & $N / A$ & $\mathrm{~N} / \mathrm{A}$ & $N / A$ & $\mathrm{~N} / \mathrm{A}$ & $N / A$ & $M / A$ & $M / A$ & $N / A$ & $M / A$ & $N / A$ & $N / A$ & $N / A$ & $M / A$ \\
\hline 8CSSP1 & $N / A$ & $N / A$ & $N / A$ & $M / A$ & $N / A$ & $N / A$ & $N / A$ & $N / A$ & $N / A$ & $N / A$ & $N / A$ & $M / A$ & $M / A$ \\
\hline 8DSSPI & $N / P$ & $N / A$ & $N / A$ & $N / A$ & $N / A$ & $N / A$ & $N / A$ & $N / A$ & $N / A$ & $N / A$ & $N / A$ & $\mathbf{N} / \mathbf{A}$ & $N / A$ \\
\hline BAMRNA & $N / A$ & $N / A$ & $M / A$ & $N / A$ & $N / A$ & $N / R$ & $N / A$ & $N / P$ & $\mathbf{N} / \mathrm{A}$ & $N / A$ & $N / A$ & $N / A$ & $\mathbf{N} / \mathbf{A}$ \\
\hline BBHRNA & $M / A$ & $N / R$ & $N / A$ & $M / A$ & $N / A$ & $N / A$ & N/A & $\mathbf{N} / \mathbf{A}$ & $N / A$ & $N / A$ & $N / P$ & $N / A$ & $N / A$ \\
\hline BCHRNA & $N / A$ & N/A & $\mathbf{M} / \mathrm{A}$ & $N / A$ & $M / A$ & $N / A$ & $N / A$ & $N / A$ & $N / A$ & $N / A$ & $N / A$ & $N / A$ & $N / A$ \\
\hline BDHRNA & $N / A$ & $N / A$ & $N / A$ & N/A & $N / A$ & $N / A$ & N/A & $M / A$ & N/A & N/A & N/ノA & $N / A$ & $N / P$ \\
\hline BEHRNA & $N / A$ & $M / A$ & $N / A$ & $N / A$ & $N / A$ & $M / A$ & N/A & $N / A$ & $N / A$ & $W / A$ & $N / A$ & $N / A$ & $N / R$ \\
\hline BFHRNA & $N / A$ & $\mathrm{~N} / \mathrm{A}$ & $\mathrm{M} / \mathrm{A}$ & $N / A$ & $N / P$ & $N / A$ & $\mathrm{~N} / \mathrm{A}$ & $N / A$ & $N / A$ & $N / A$ & $W / A$ & $N / A$ & $M / A$ \\
\hline BGHRNA & N/A & $\mathrm{N} / \mathrm{A}$ & $N / A$ & $N / A$ & $N / P$ & $N / A$ & $N / A$ & $N / A$ & $N / A$ & $M / A$ & $N / A$ & $N / A$ & $N / A$ \\
\hline CBSCRAM & $N / A$ & $N / A$ & $N / A$ & $N / A$ & $N / A$ & $N / A$ & N/R & $N / A$ & $N / A$ & $N / A$ & $N / A$ & $N / A$ & $N / A$ \\
\hline GHR* & NS & NS & NS & $N / A$ & $N / A$ & NS & NS & MS & NS & MS & NS & NS & NS \\
\hline WPE* & [Zero & events in & relevant & time $s$ & le] & & & & & & & & \\
\hline RERLE* & [Zera & events in & relevant & time $s$ & le] & & & & & & & & \\
\hline BACKECN* & $N / P$ & $N / A$ & $N / A$ & $N / A$ & $M / A$ & $N / A$ & $H / A$ & $N / A$ & $\mathrm{~N} / \mathrm{A}$ & $\mathbf{N} / \mathrm{A}$ & $N / A$ & W/A & $\mathrm{N} / \mathrm{A}$ \\
\hline WRONGT/U* & $N / A$ & $\mathrm{~N} / \mathrm{A}$ & $\mathrm{N} / \mathrm{A}$ & $N / P$ & $N / A$ & $\mathrm{~N} / \mathrm{A}$ & $\mathrm{N} / \mathrm{A}$ & $N / A$ & $N / A$ & $N / A$ & $N / R$ & $N / A$ & $N / A$ \\
\hline
\end{tabular}

ESFACT* [Zero events in relevant time scale]

Nate: Indicators marked with an "*" are SAIC indicators 
Significant Maintenance Indicator/Measure of Safety Relationships

\begin{tabular}{|c|c|c|c|}
\hline $\begin{array}{l}\text { Indicator } \\
=======\end{array}$ & $\begin{array}{l}\text { Measure of Safety } \\
=============\end{array}$ & $\begin{array}{l}\text { Time Shift } \\
========\end{array}$ & $\begin{array}{l}\text { Conf i dence } \\
========\end{array}$ \\
\hline GDEF IC & AUAIL & +2 & $85 \%$ \\
\hline 9DBOPMT & FRATE & +1 & $82 \%$ \\
\hline CSCRAM & FOR & +1 & $89 \%$ \\
\hline CSCRAM & FRRTE & +1 & $83 \%$ \\
\hline
\end{tabular}


Performance Indicator Analysis
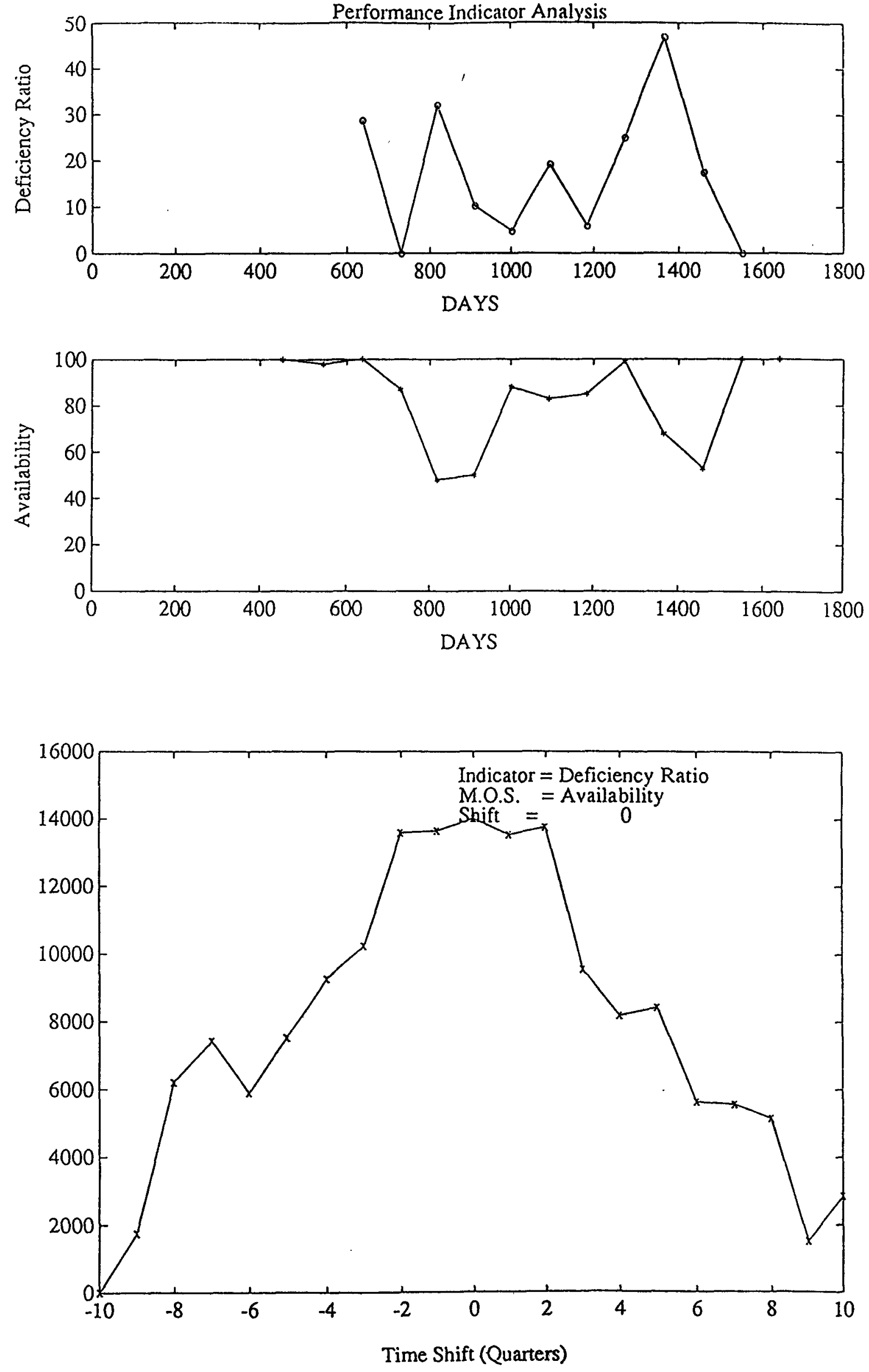

B-70 

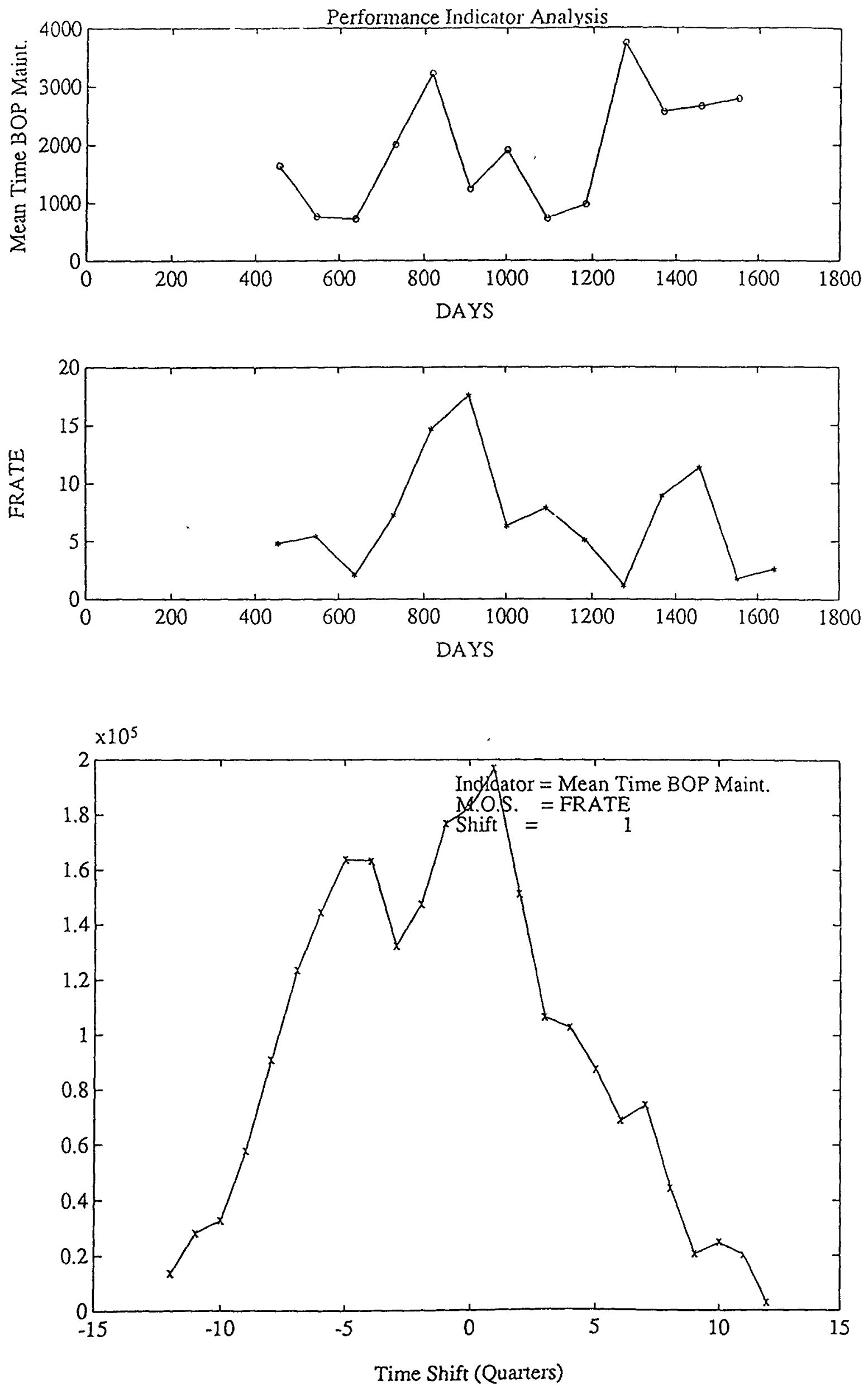

B-71 

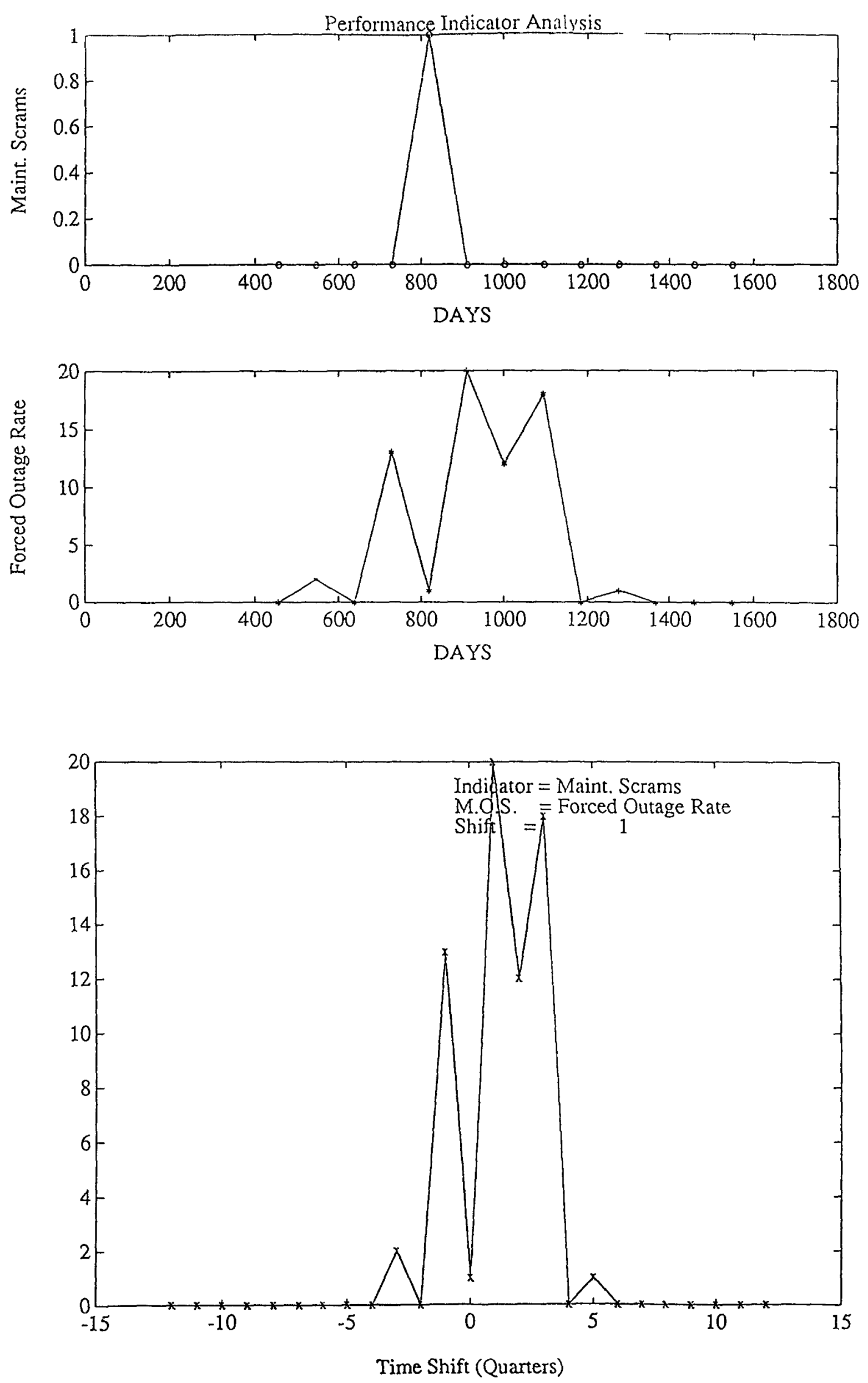

B-72 

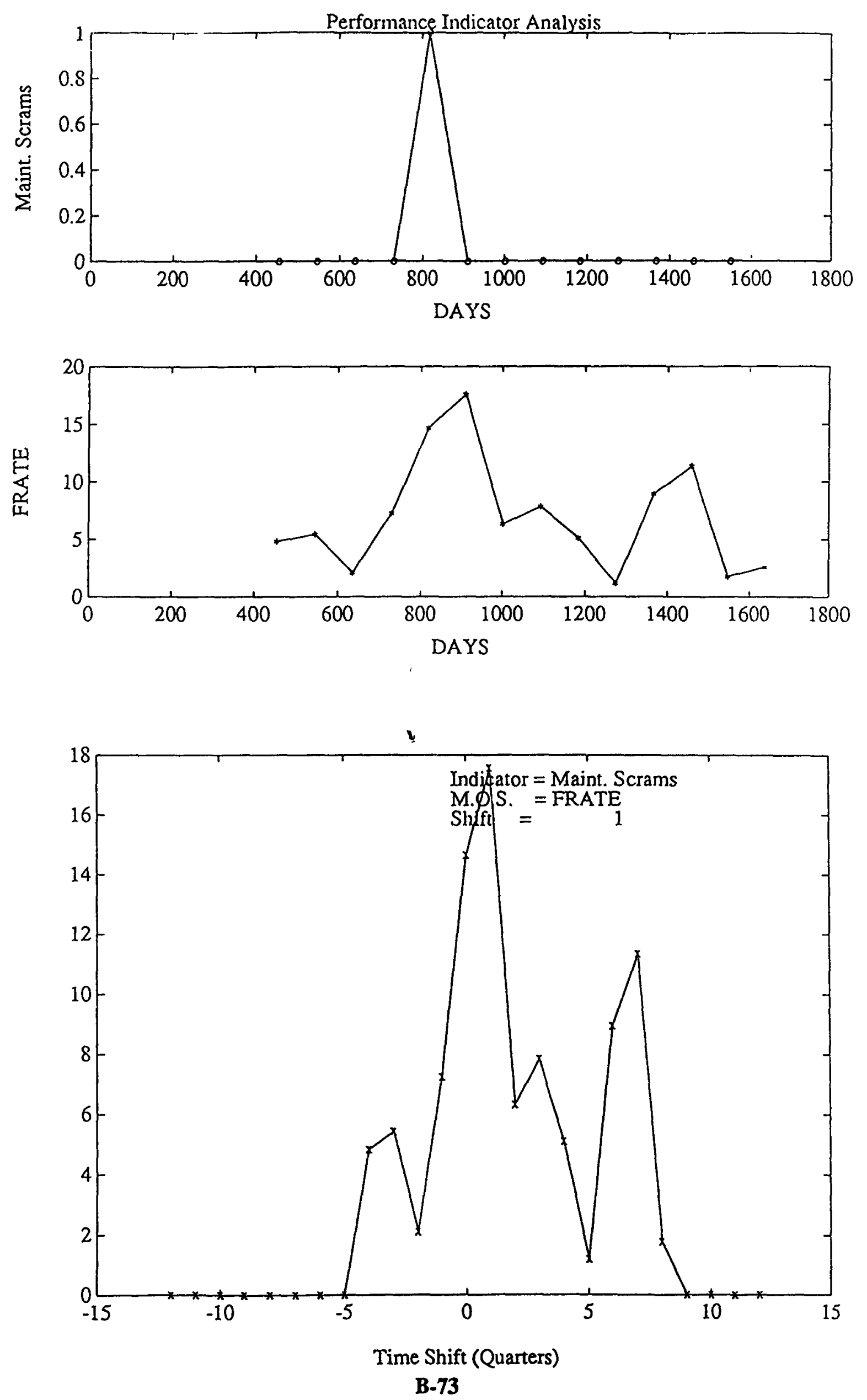

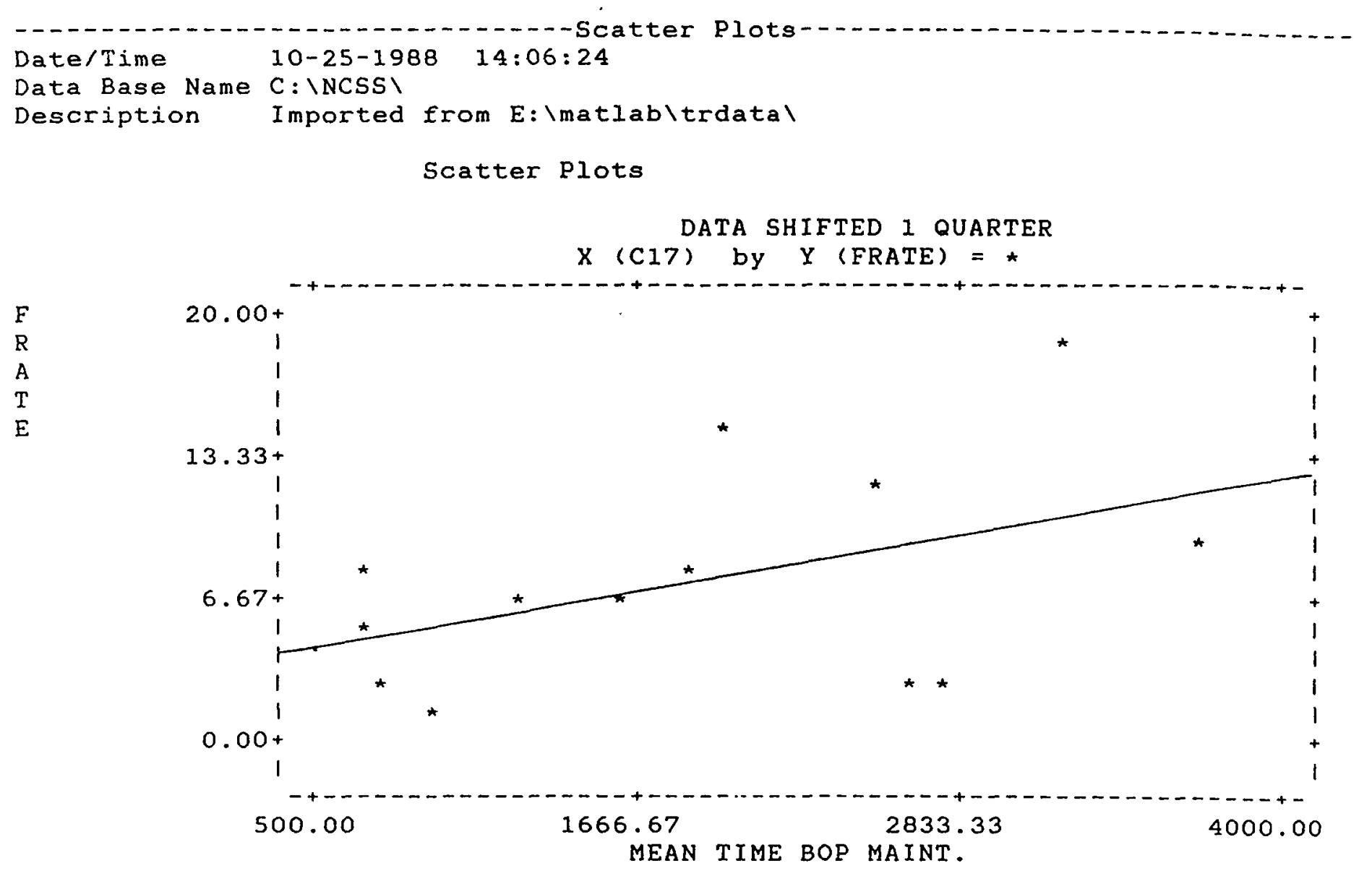

1. Accounting Report

$\begin{array}{lllrr}\text { Number of points below minimum } & (X): & 0 & (Y): & 0 \\ \text { Number of points above maximum } & (X): & 0 & (Y): & 0 \\ \text { Number of points with missing values }(X): & 1 & (Y): & 0 \\ \text { Number of points plotted } & & : & 13 & \end{array}$




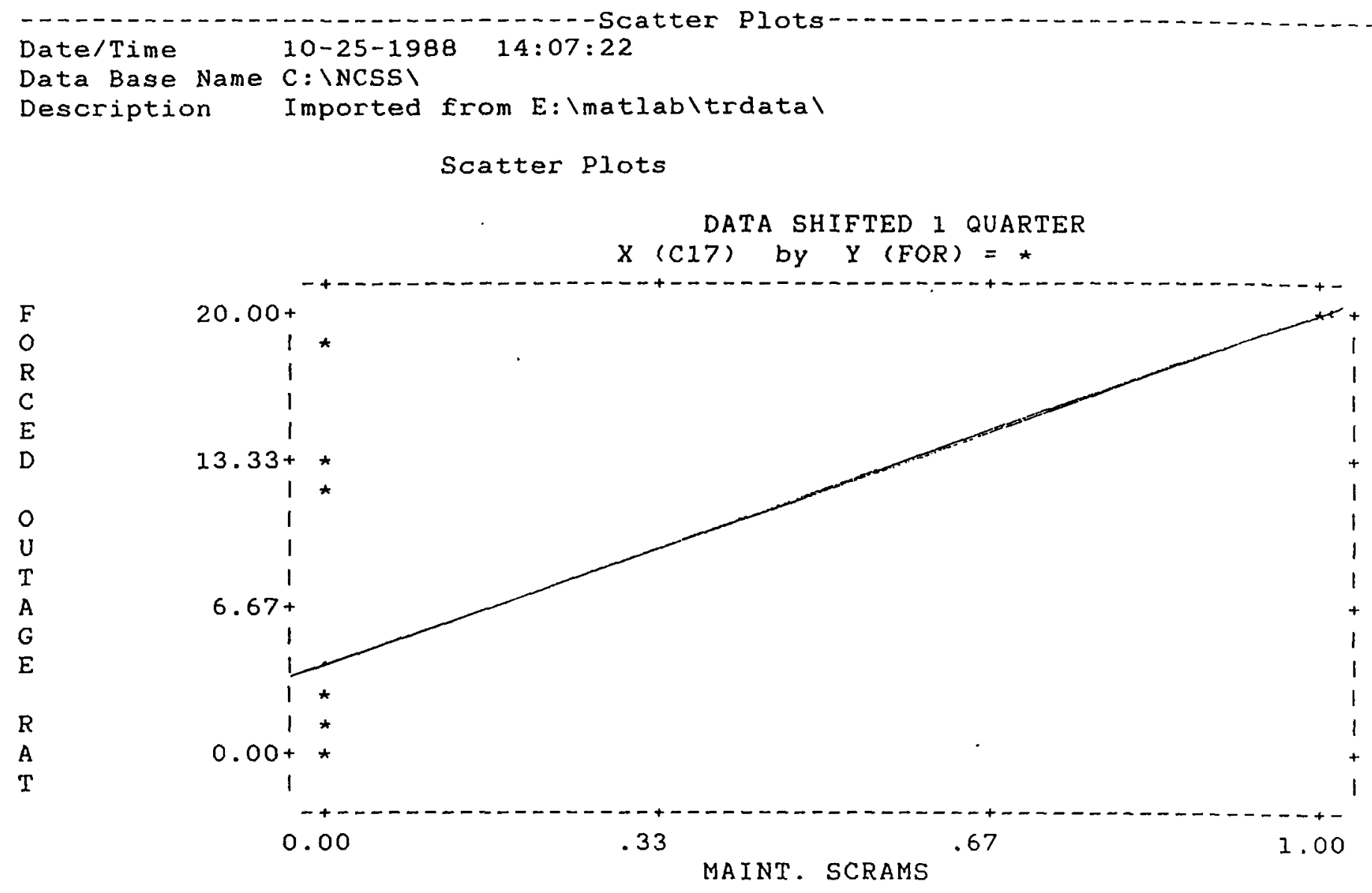

Row Accounting Report

$\begin{array}{lllllll}\text { Number of points below minimum } & (X): & 0 & (Y): & 0 \\ \text { Number of points above maximum } & (X): & 0 & (Y): & 0 \\ \text { Number of points with missing values }(X): & 1 & (Y): & 1 \\ \text { Number of points plotted } & & : & 12 & & & \end{array}$




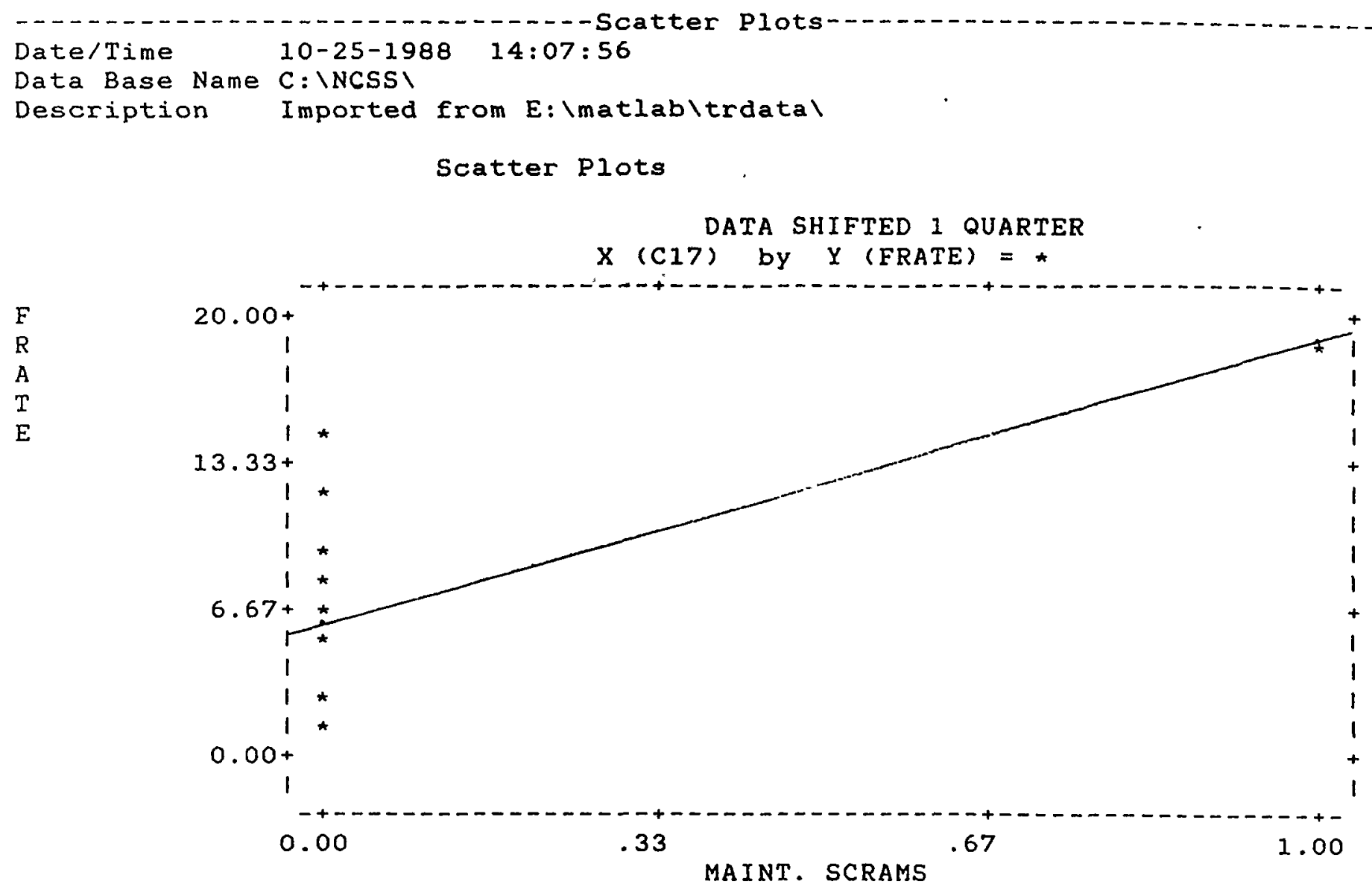

Row Accounting Report

$\begin{array}{lllrr:}\text { Number of points below minimum } & (X): & 0 & (Y): & 0 \\ \text { Number of points above maximum } & (X): & 0 & (Y): & 0 \\ \text { Number of points with missing values }(X): & 1 & (Y): & 0 \\ \text { Number of points plotted } & & : & 13 & \end{array}$




\section{Detailed Results for Plant $\mathbf{H}$}


Maintenance Indicators vs. Measures of Safety

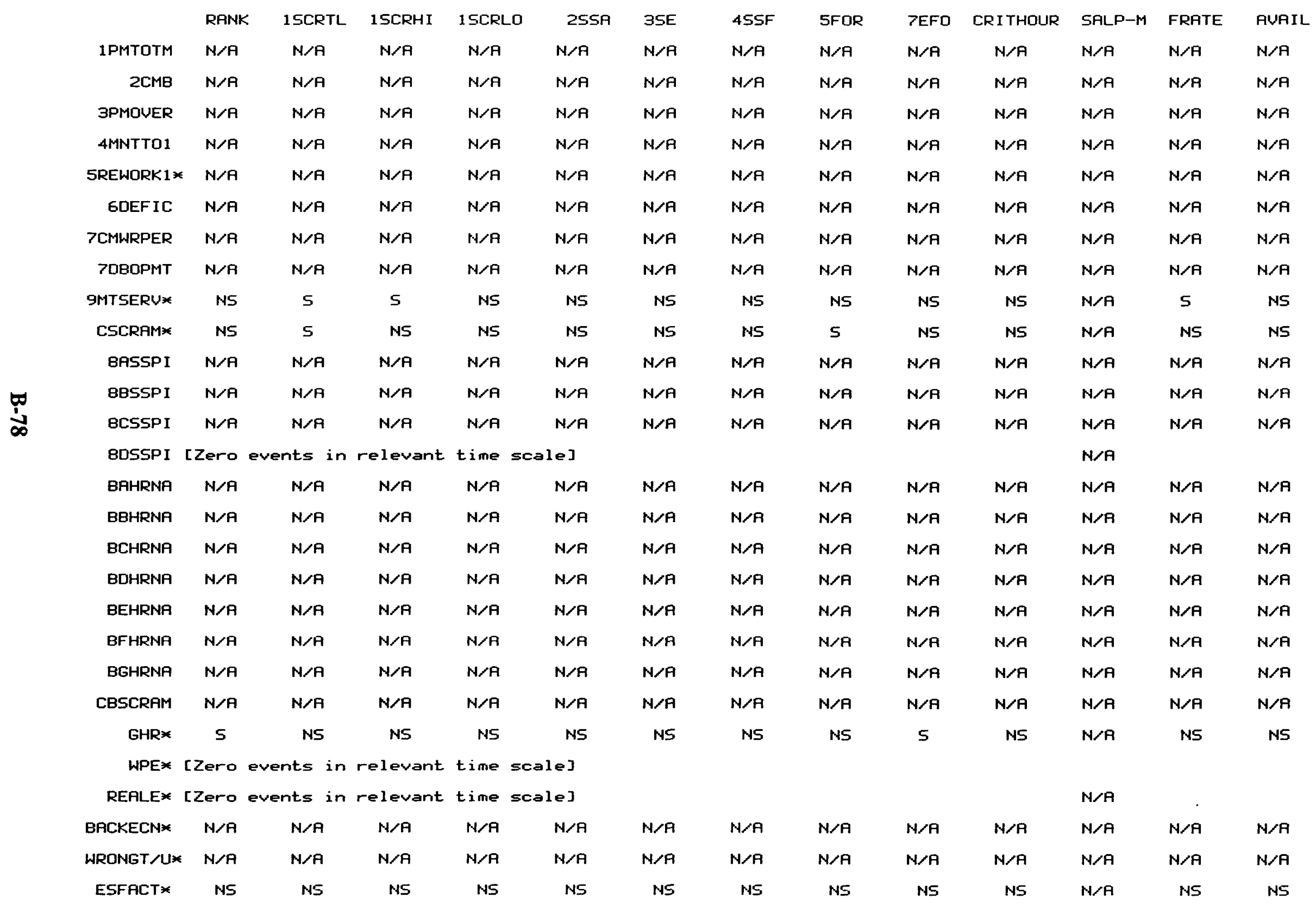

Note: Indicators marked with an "*" are SAIC indicators 
Significant Maintenance Indicator/Measure of Safety Relationships

Indicator $== \pm===$
gMTSERU

SMTSERU

SMTSERV

GHR

GHR

CSCRAM

CSCRAM

Measure of Safety
$=============$
FRATE
SCRHI
SCRTL
RANK
EFO
SCRTL
FOR

\begin{tabular}{|c|c|}
\hline $\begin{array}{c}\text { Time Shift } \\
======= \\
0\end{array}$ & $\begin{array}{c}\text { Conf idence } \\
======== \\
80 \%\end{array}$ \\
\hline+1 & $96 \%$ \\
\hline+1 & $95 \%$ \\
\hline 0 & $90 \%$ \\
\hline 0 & $80 \%$ \\
\hline 0 & $82 \%$ \\
\hline 0 & $82 \%$ \\
\hline
\end{tabular}



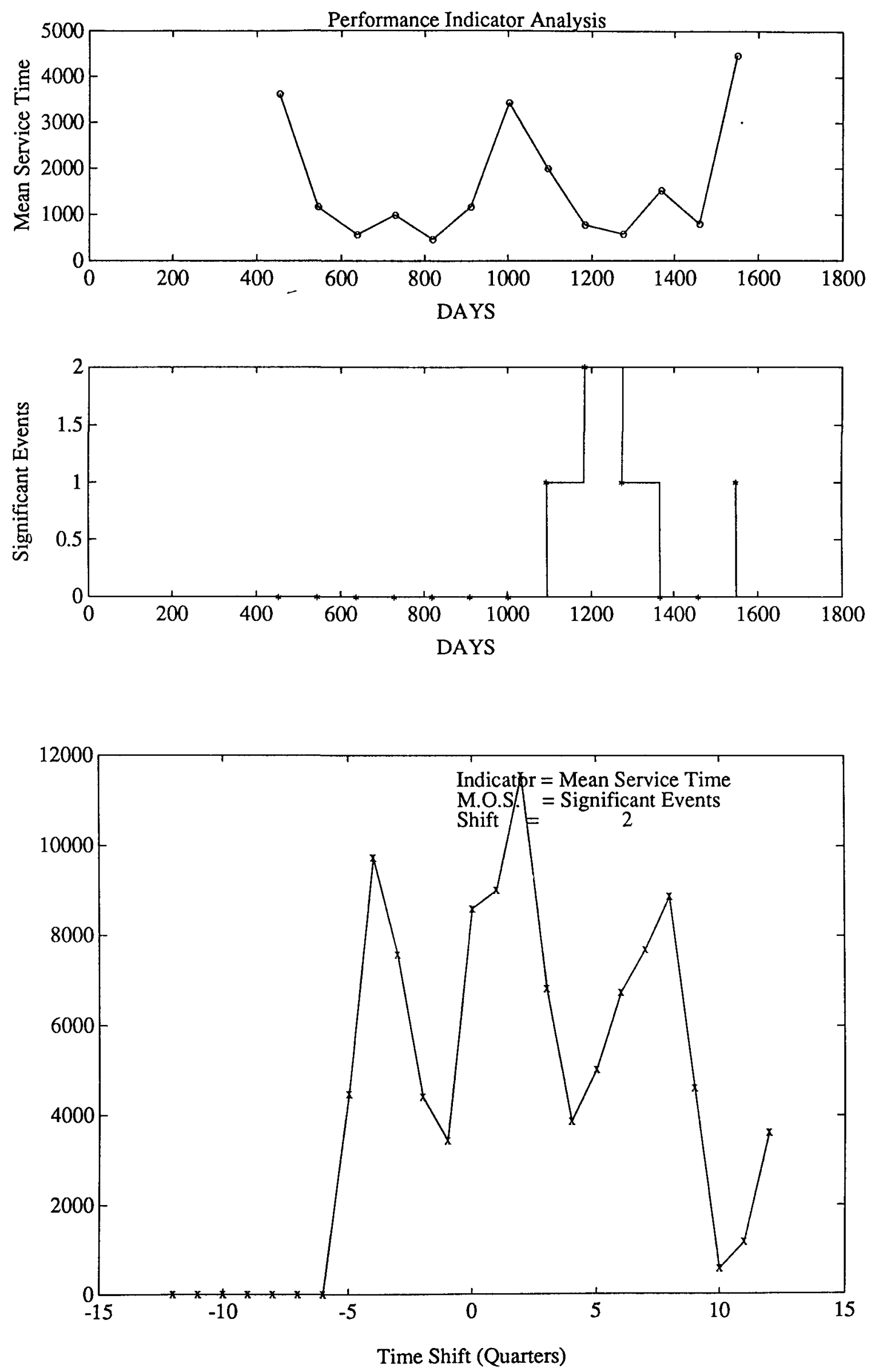

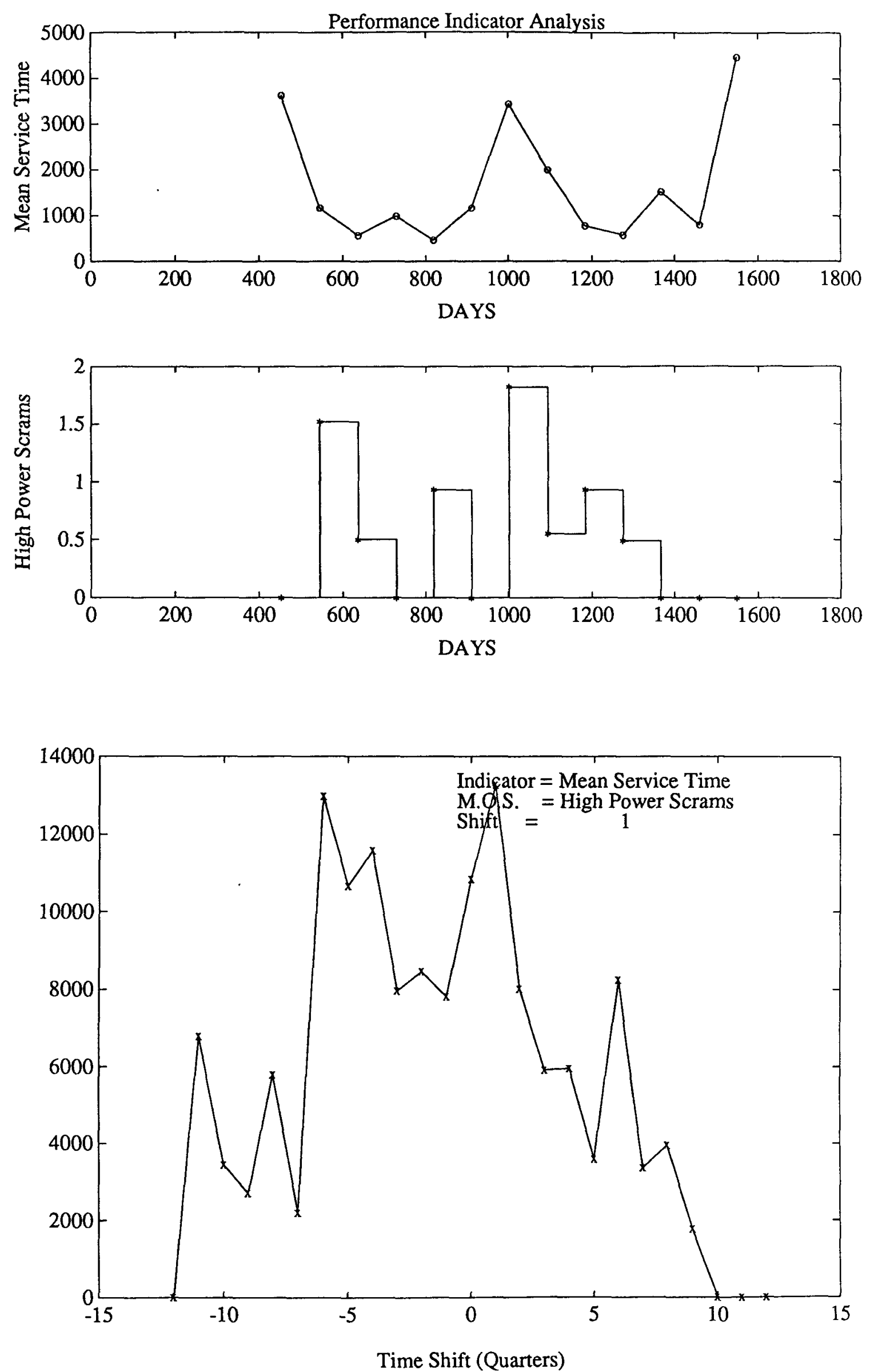

B-81 

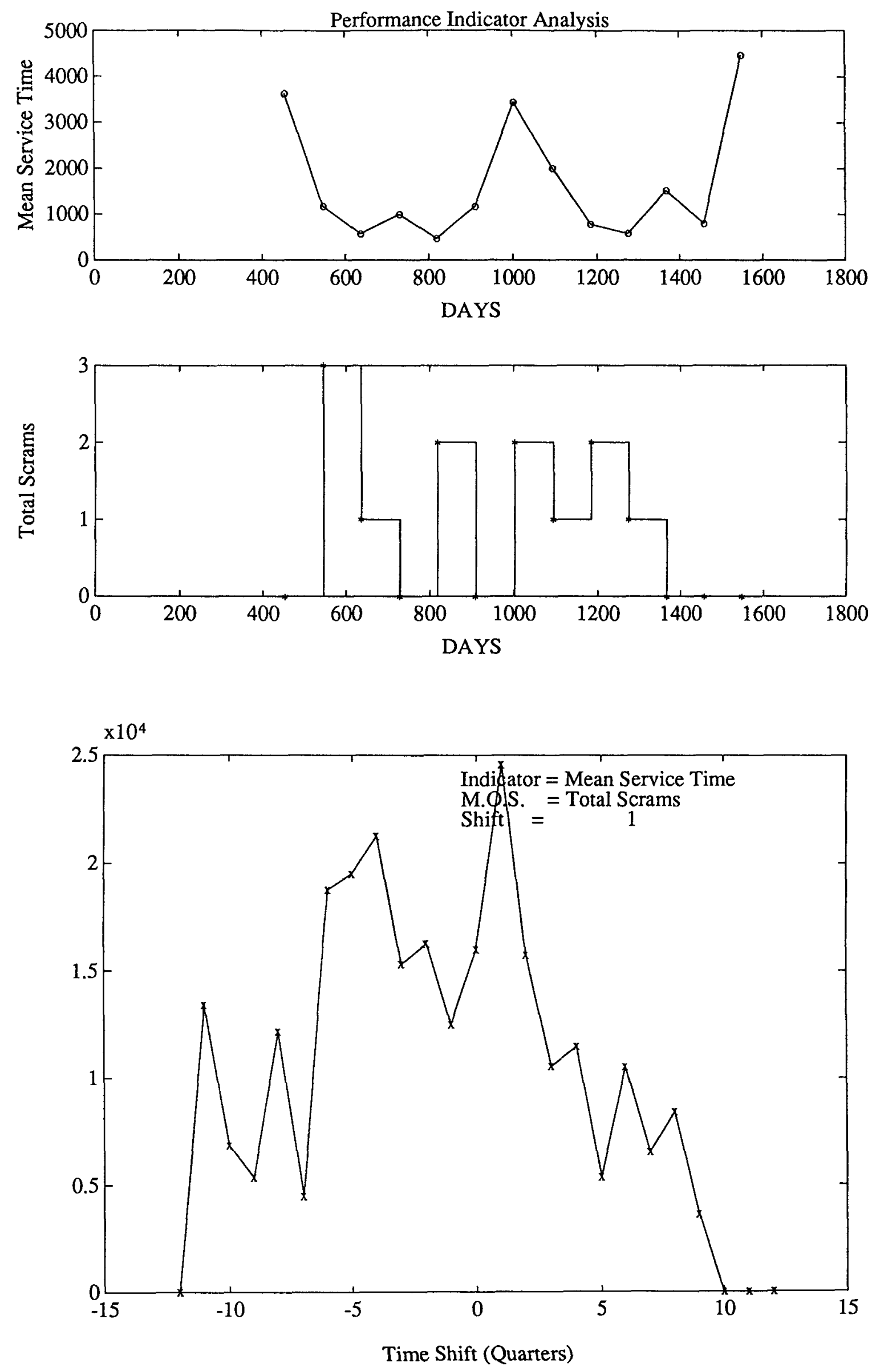

B-82 

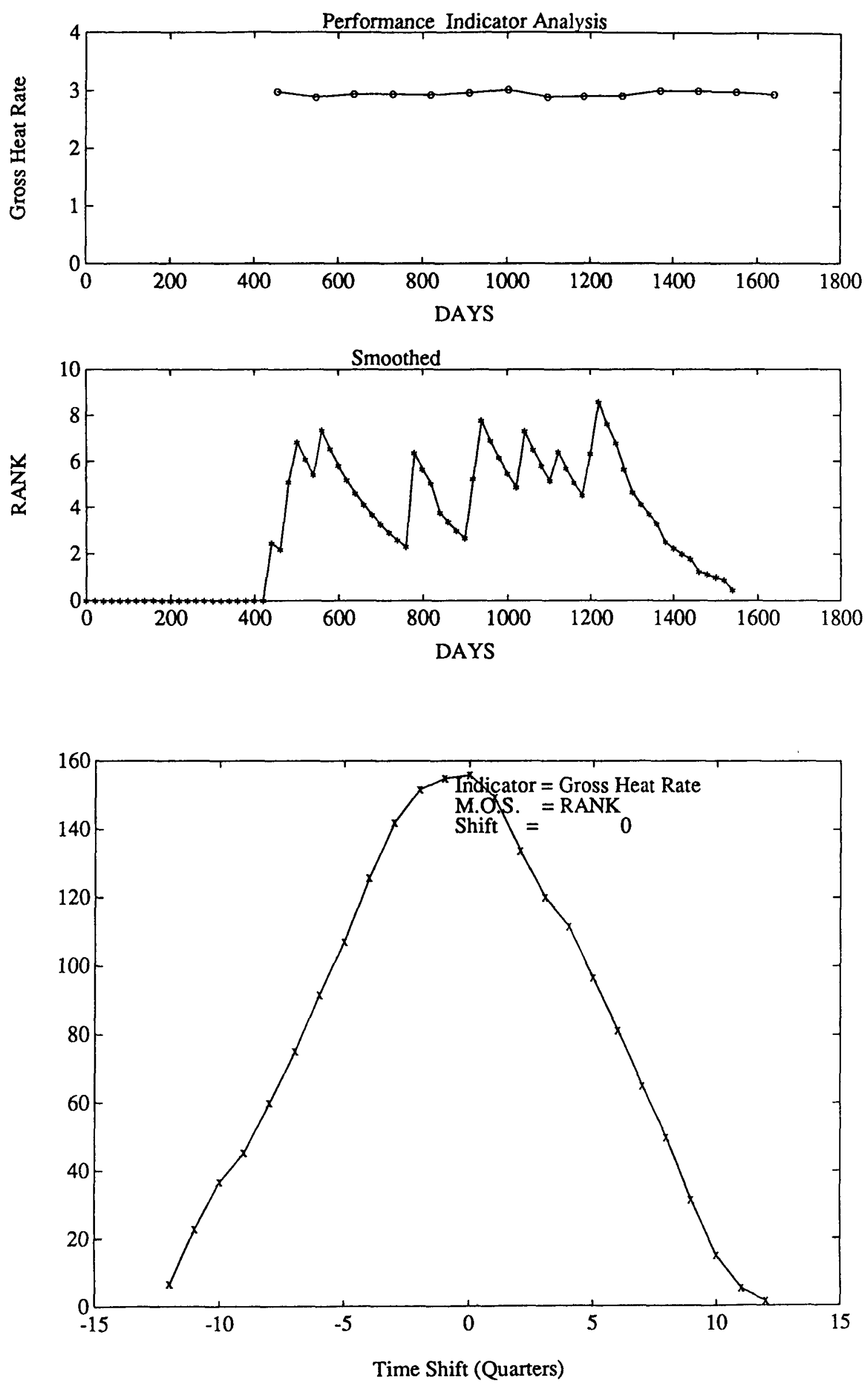

B-83 

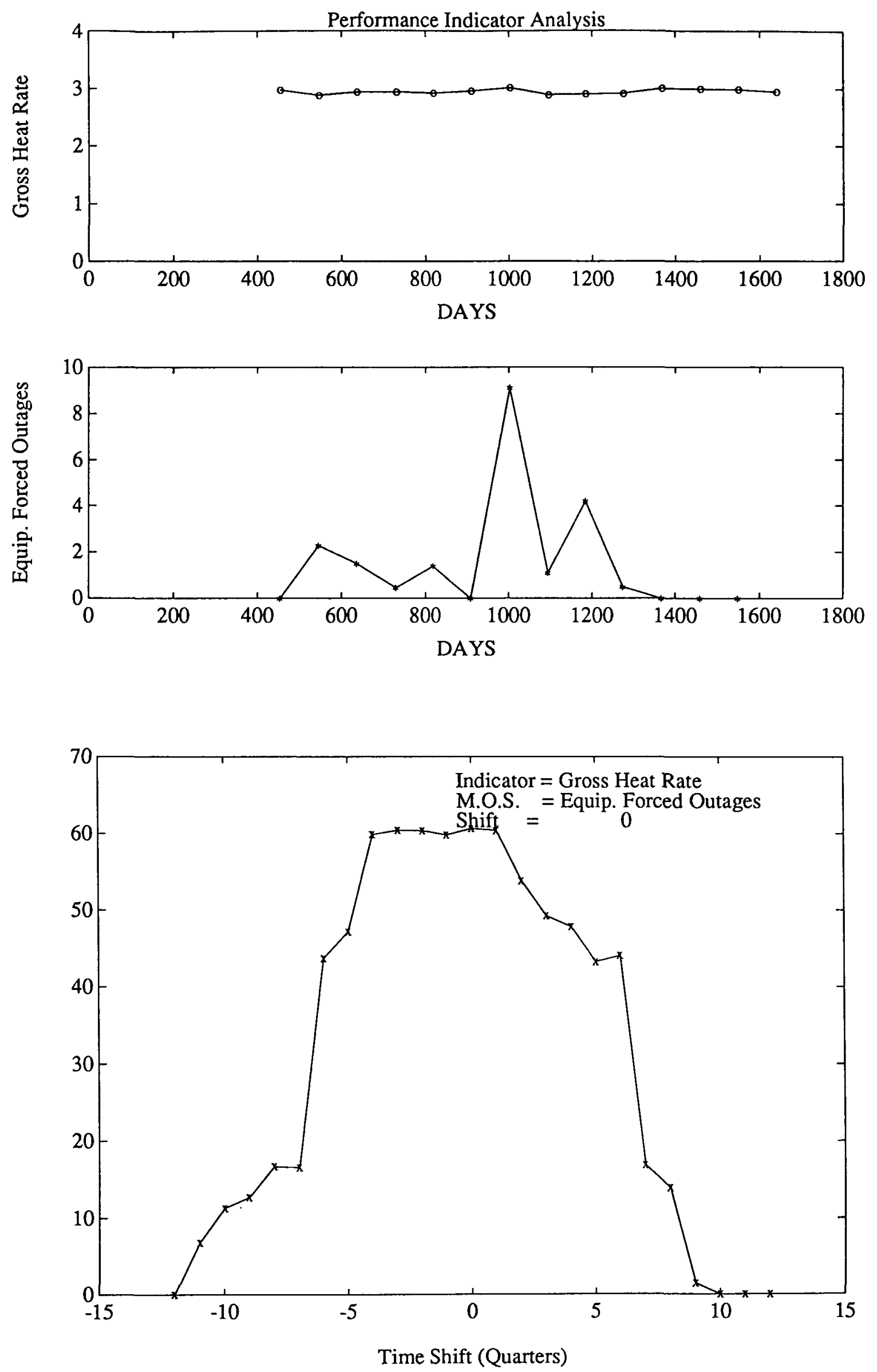

B-84 

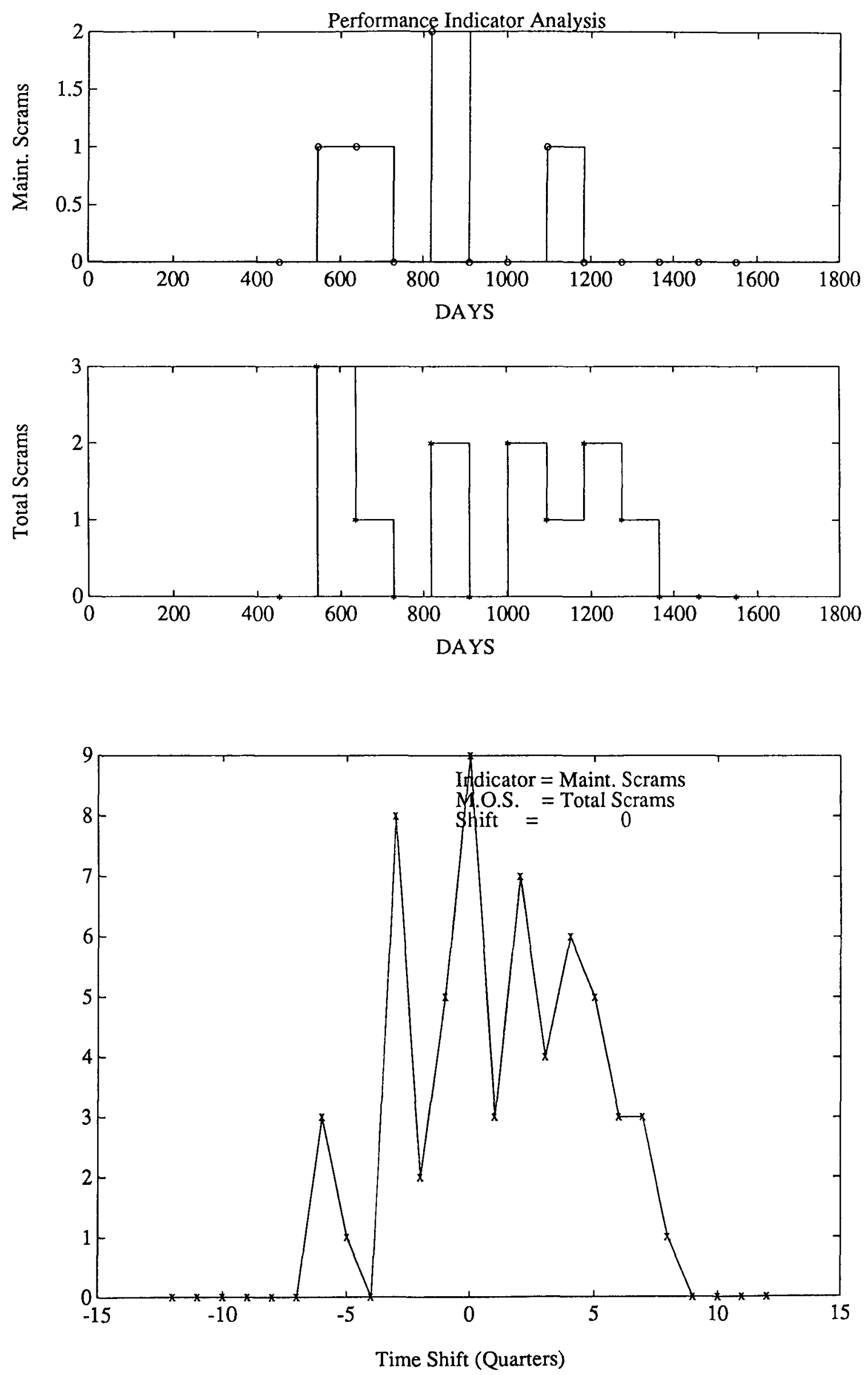

B-85 

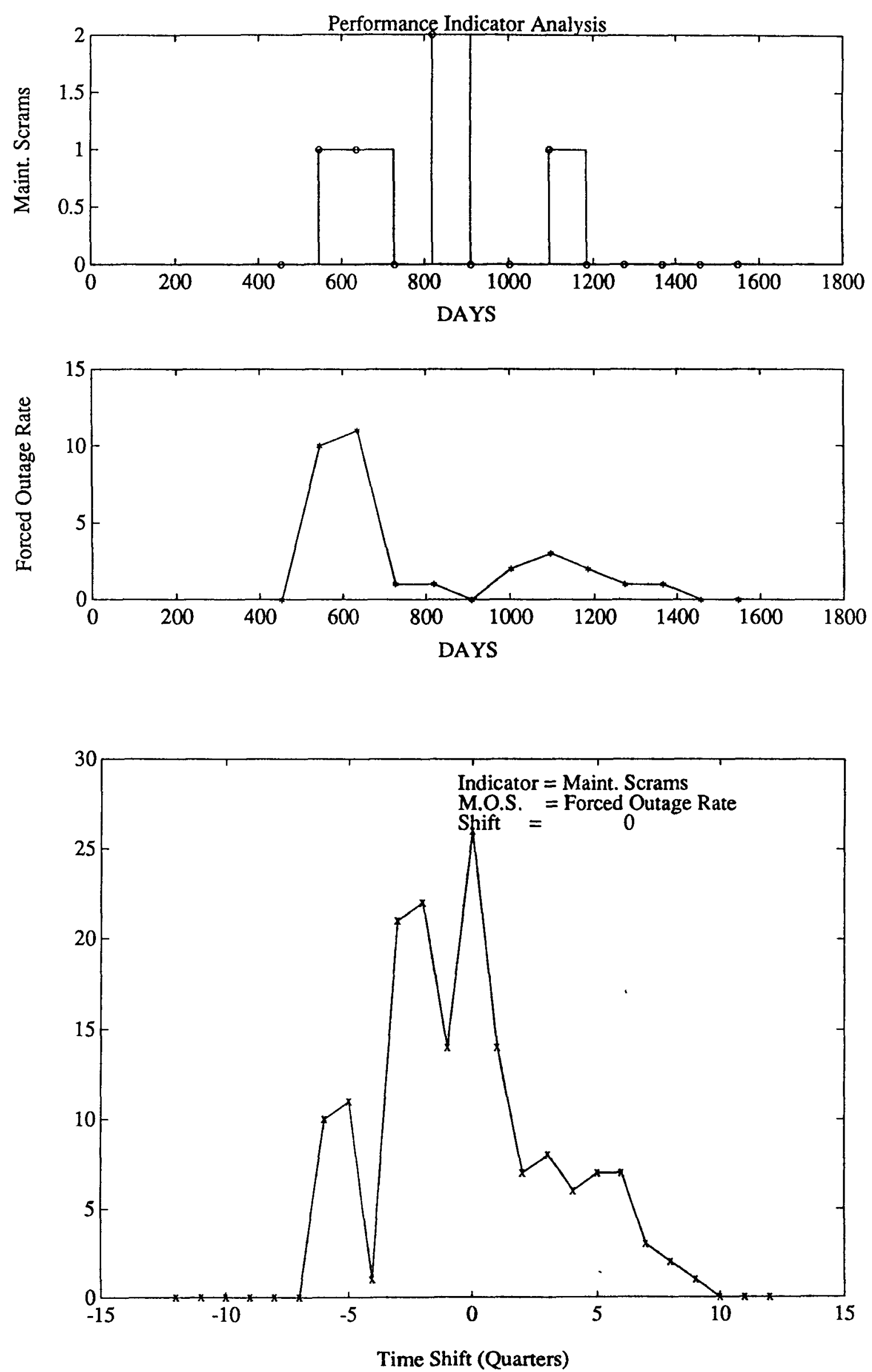

B-86 


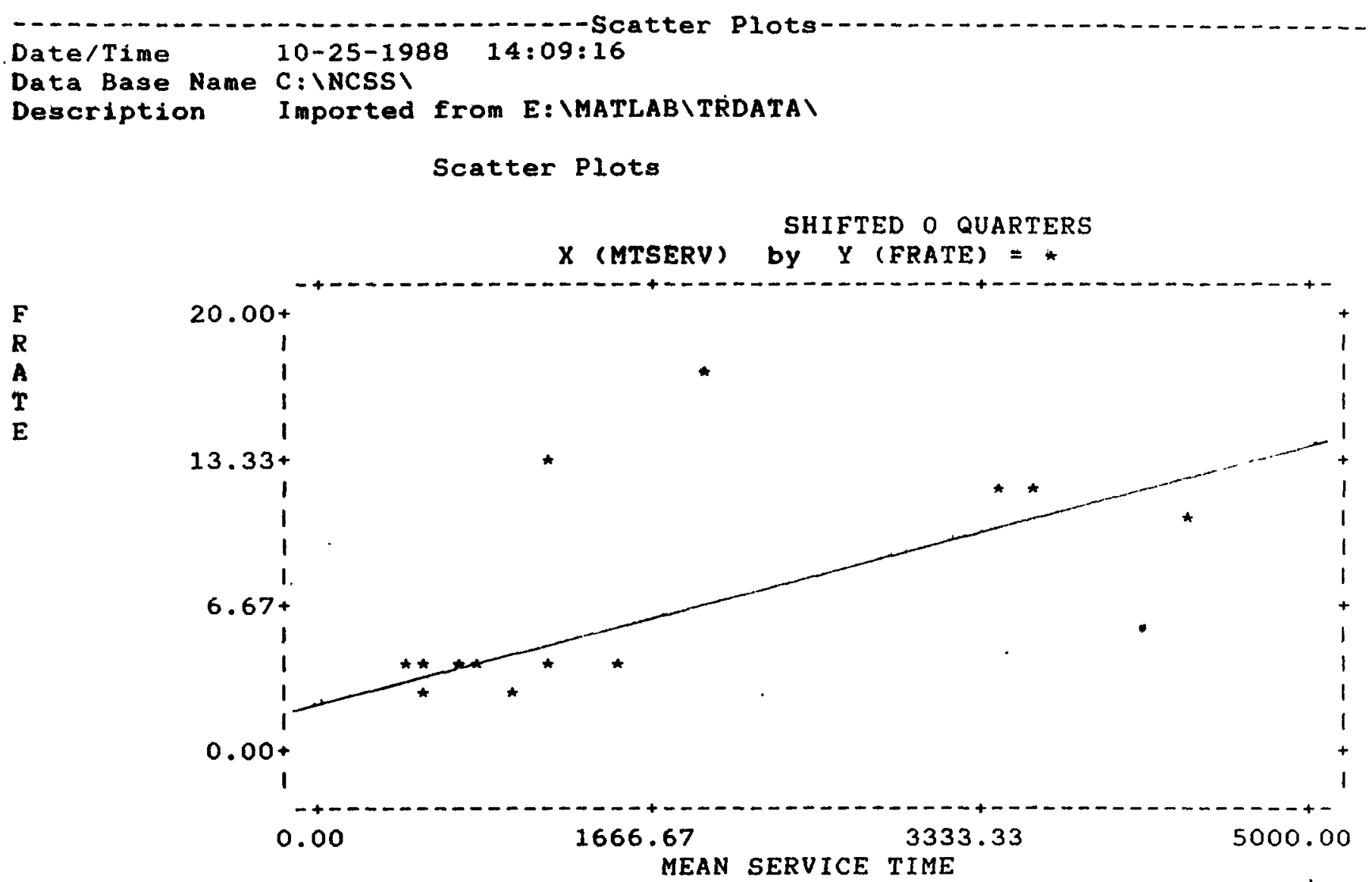

L.w Accounting Report

$\begin{array}{lllrlll}\text { Number of points below minimum } & (X): & 0 & (Y): & 0 \\ \text { Number of points above maximum } & (X): & 0 & (Y): & 0 \\ \text { Number of points with missing values }(X): & 0 & (Y): & 0 \\ \text { Number of points plotted } & & : & 13 & & & \end{array}$




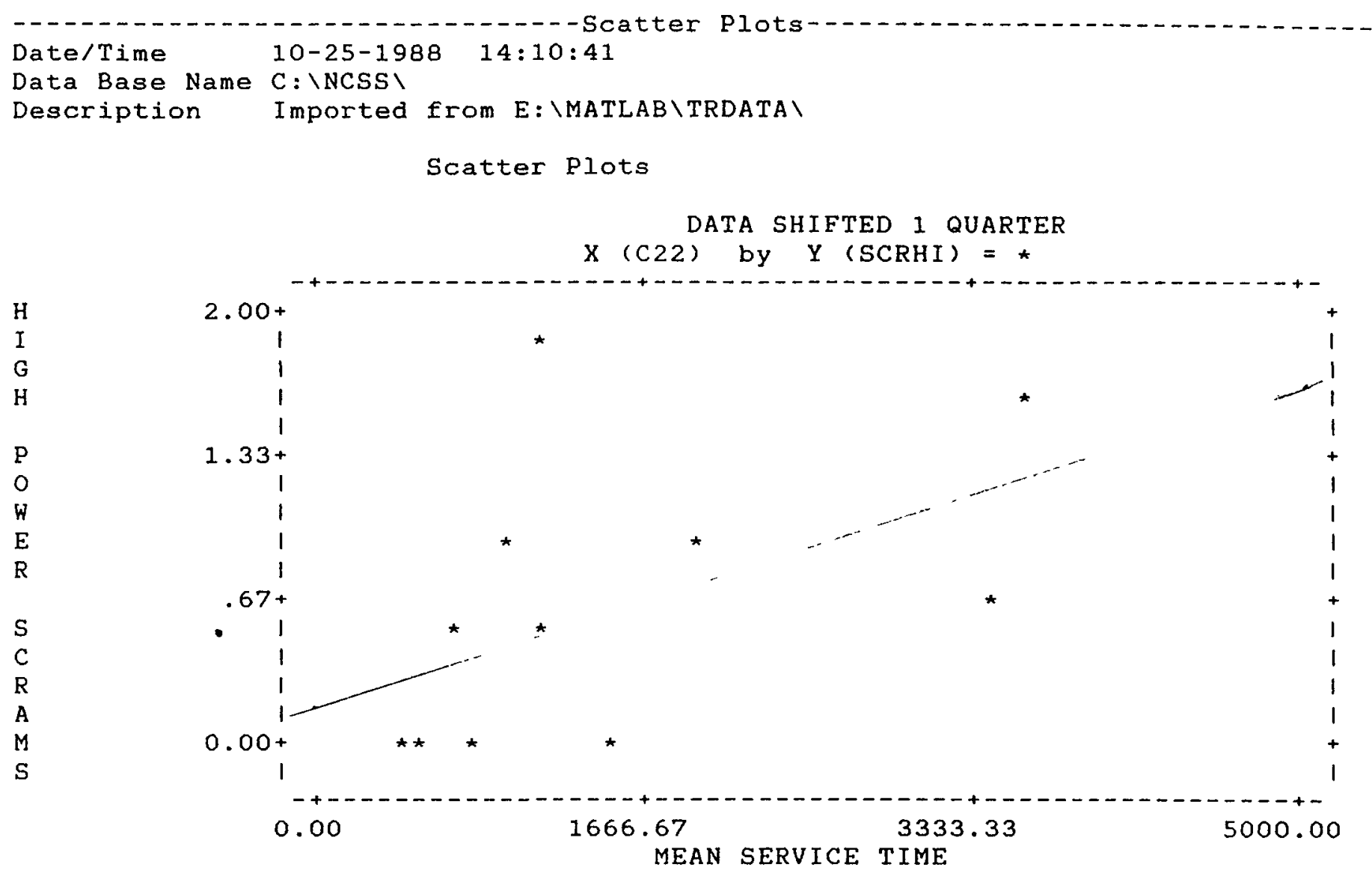

kuw Accounting Report

$\begin{array}{lllrlll}\text { Number of points below minimum } & (X): & 0 & (Y): & 0 \\ \text { Number of points above maximum } & (X): & 0 & (Y): & 0 \\ \text { Number of points with missing values }(X): & 1 & (Y): & 1 \\ \text { Number of points plotted } & & : & 12 & & & \end{array}$




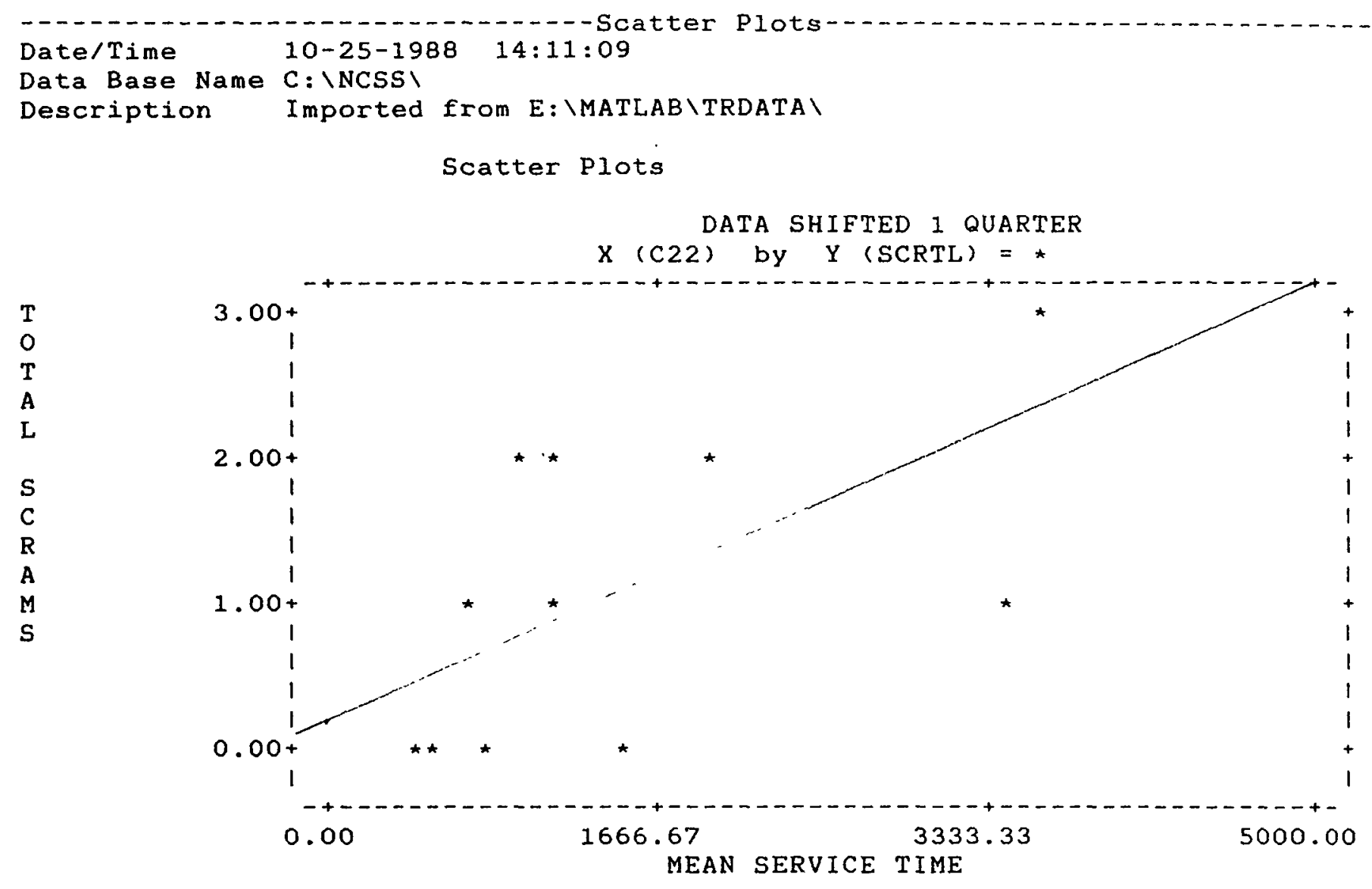

k-w Accounting Report

$\begin{array}{llrrr}\text { Number of points below minimum } & (X): & 0 & (Y): & 0 \\ \text { Number of points above maximum } & (X): & 0 & (Y): & 0 \\ \text { Number of points with missing values } & (X): & 1 & (Y): & 1 \\ \text { Number of points plotted } & : & 12 & & \end{array}$




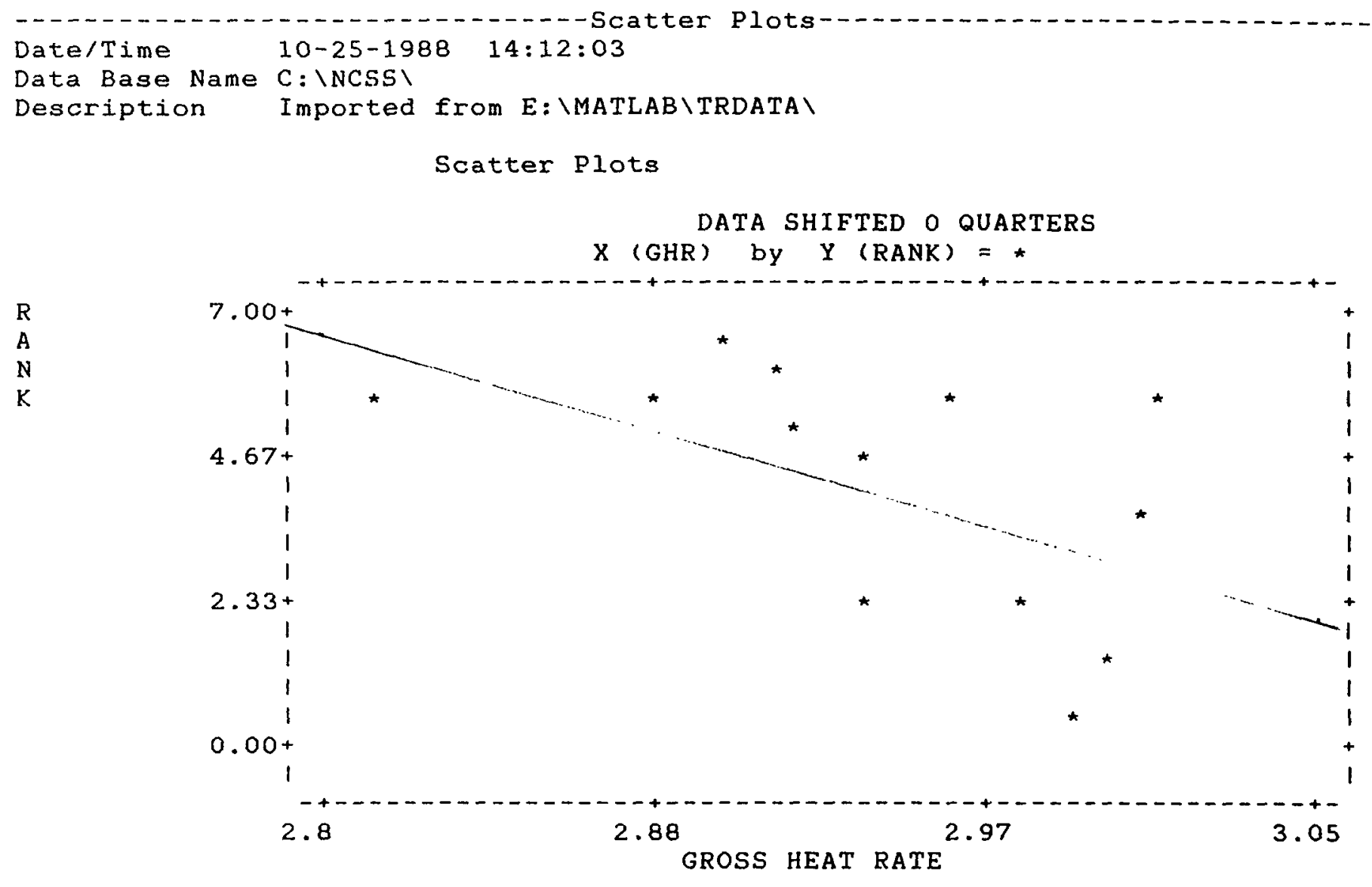

hu Accounting Report

$\begin{array}{lllrlll}\text { Number of points below minimum } & (X): & 0 & (Y): & 0 \\ \text { Number of points above maximum } & (X): & 0 & (Y): & 0 \\ \text { Number of points with missing values }(X): & 0 & (Y): & 0 \\ \text { Number of points plotted } & & : & 13 & & \end{array}$




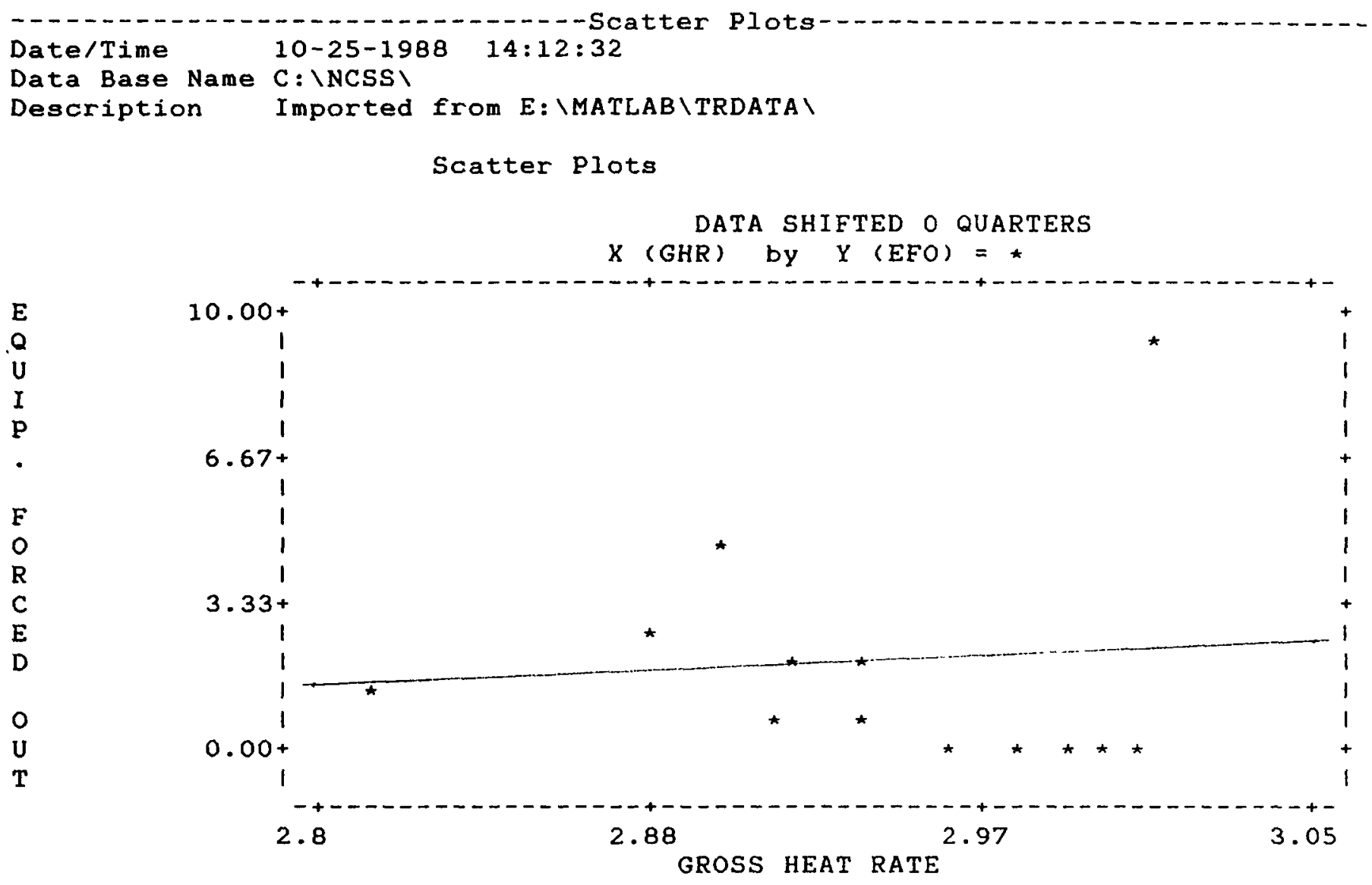

- Accounting Report

$\begin{array}{lllllll}\text { Number of points below minimum } & (X): & 0 & (Y): & 0 \\ \text { Number of points above maximum } & (X): & 0 & (Y): & 0 \\ \text { Number of points with missing values } & (X): & 0 & (Y): & 1 \\ \text { Number of points plotted } & & : & 13 & & \end{array}$




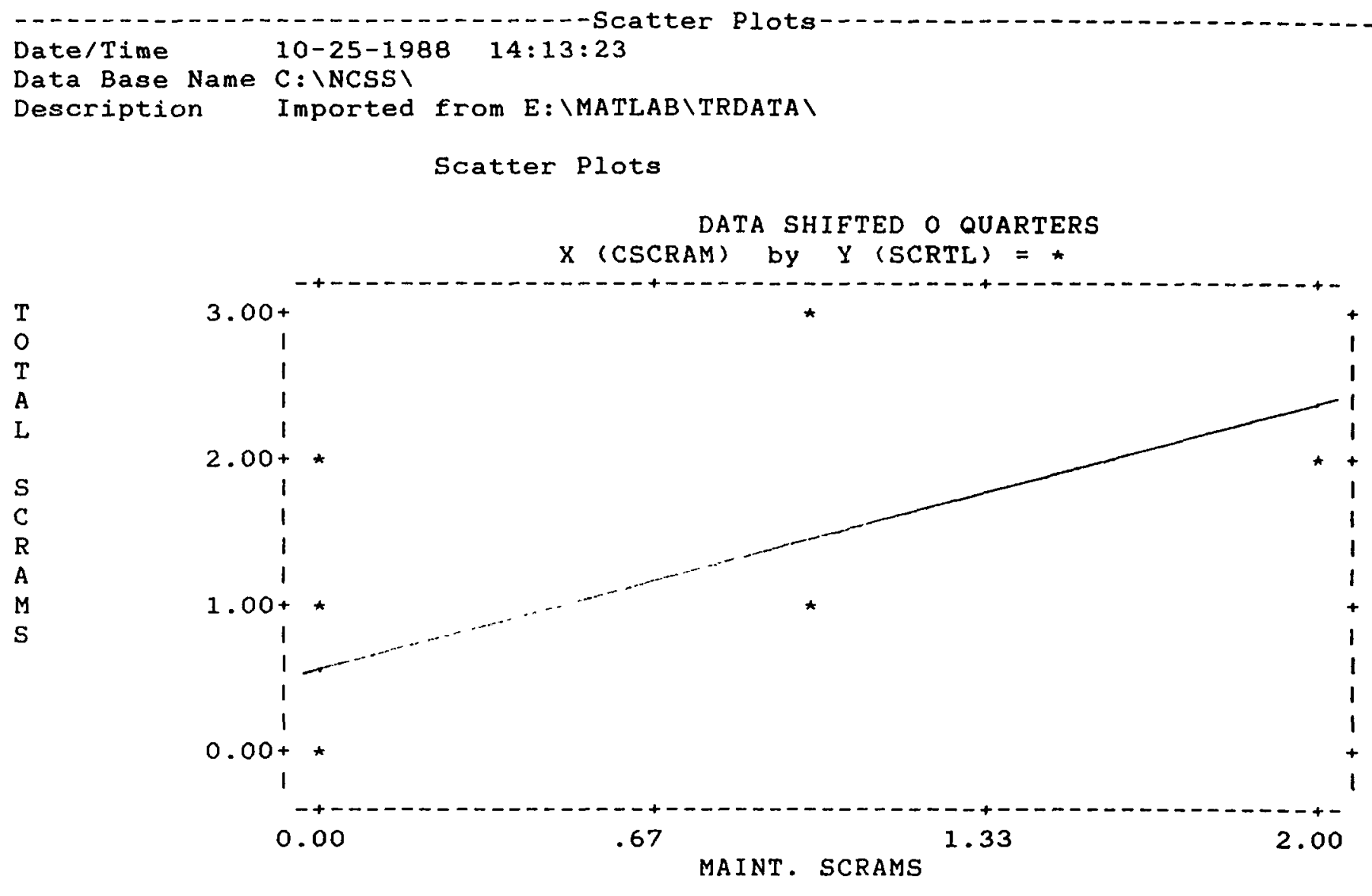

... Accounting Report

$\begin{array}{lllrlll}\text { Number of points below minimum } & (X): & 0 & (Y): & 0 \\ \text { Number of points above maximum } & (X): & 0 & (Y): & 0 \\ \text { Number of points with missing values } & (X): & 0 & (Y): & 0 \\ \text { Number of points plotted } & & : & 13 & & & \end{array}$




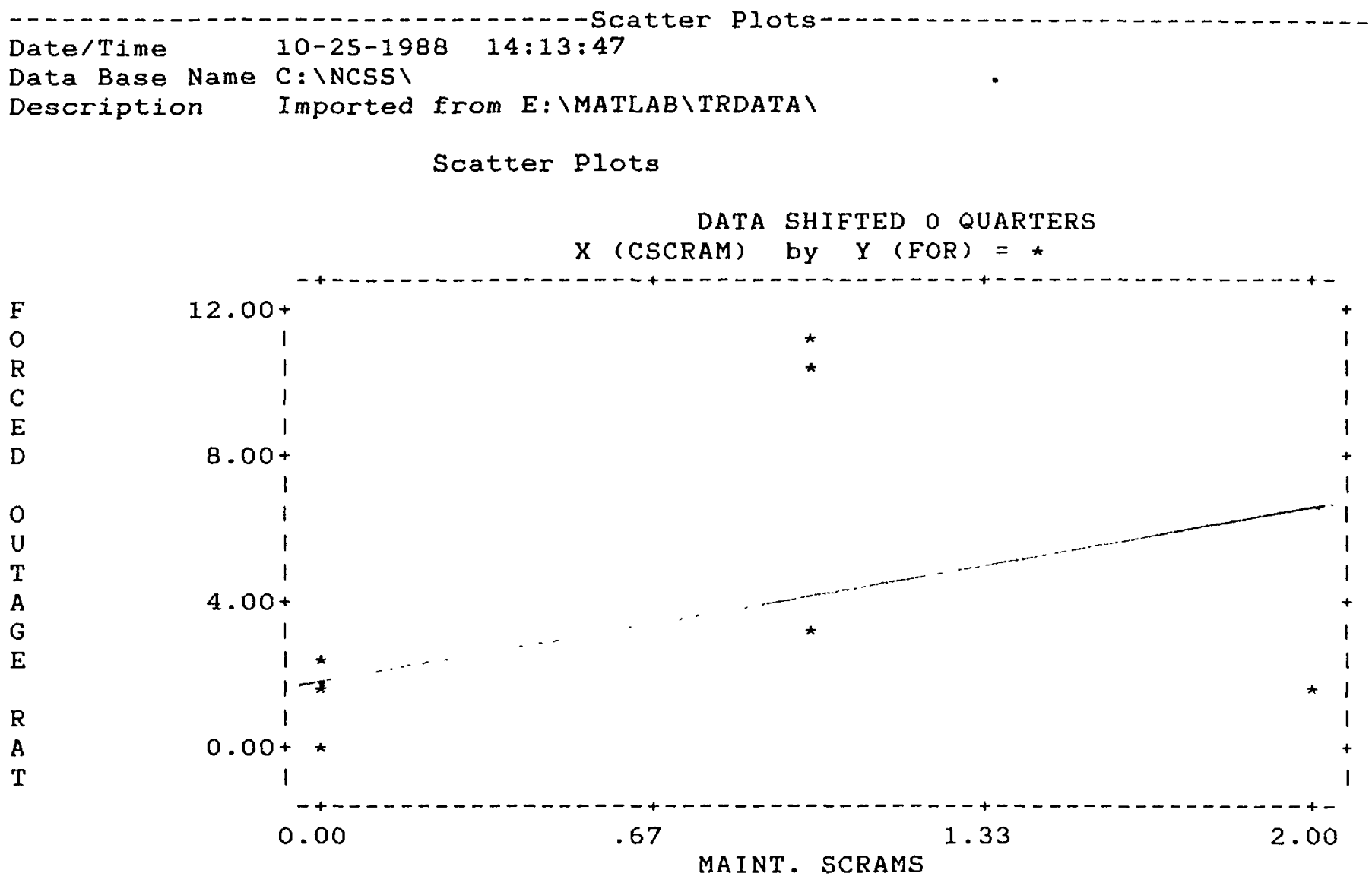

1. w Accounting Report

$\begin{array}{lllrlll}\text { Number of points below minimum } & (X): & 0 & (Y): & 0 \\ \text { Number of points above maximum } & (X): & 0 & (Y): & 0 \\ \text { Number of points with missing values }(X): & 0 & (Y): & 0 \\ \text { Number of points plotted } & & : & 13 & & & \end{array}$


Detailed Results for Plant J 
Maintenance Indicators vs. Measures of Safety

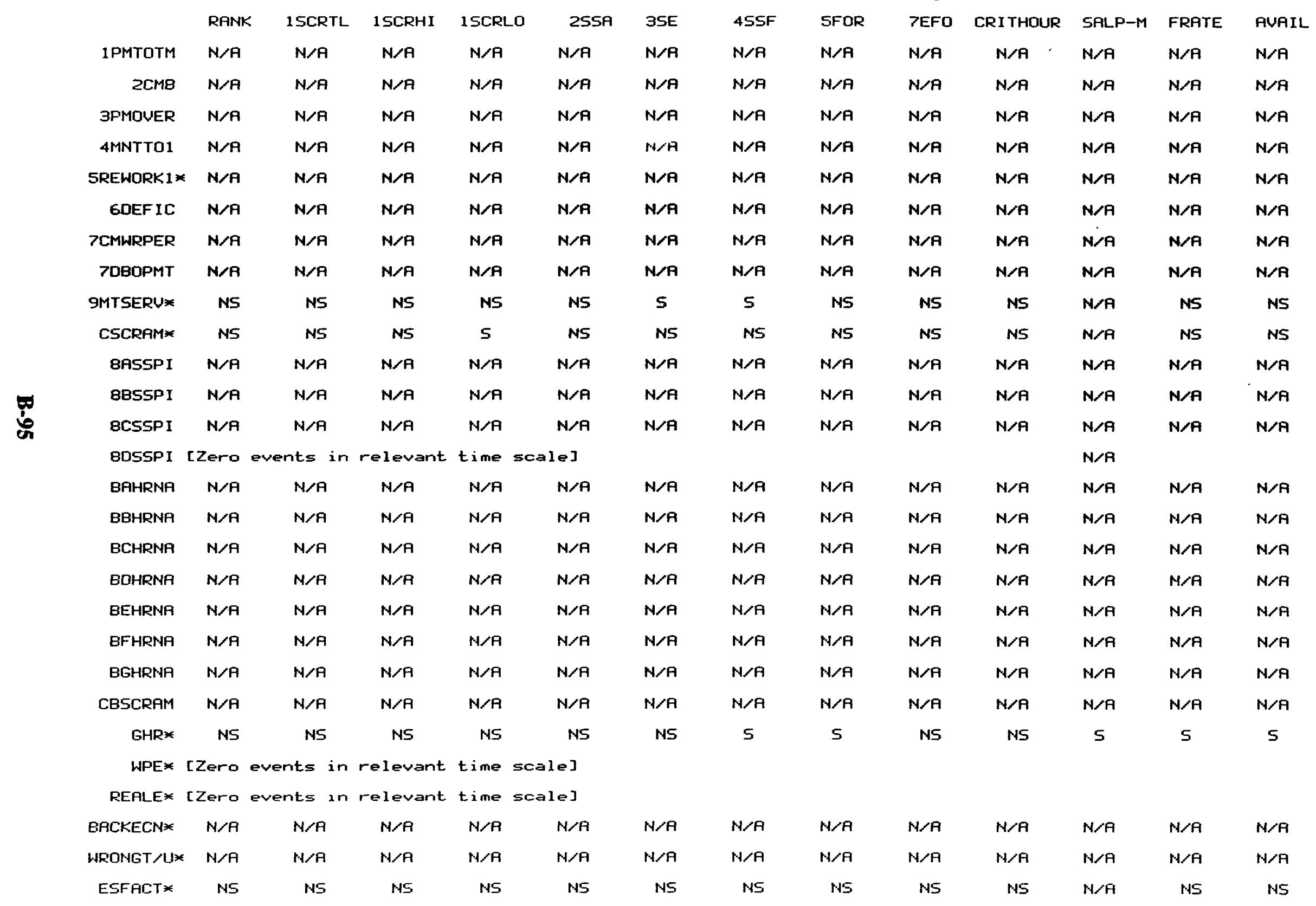

Hote: Indicators marked with an "*" are SAIC indicators 
Significant Maintenance Indicator/Measure of Safety Relationships

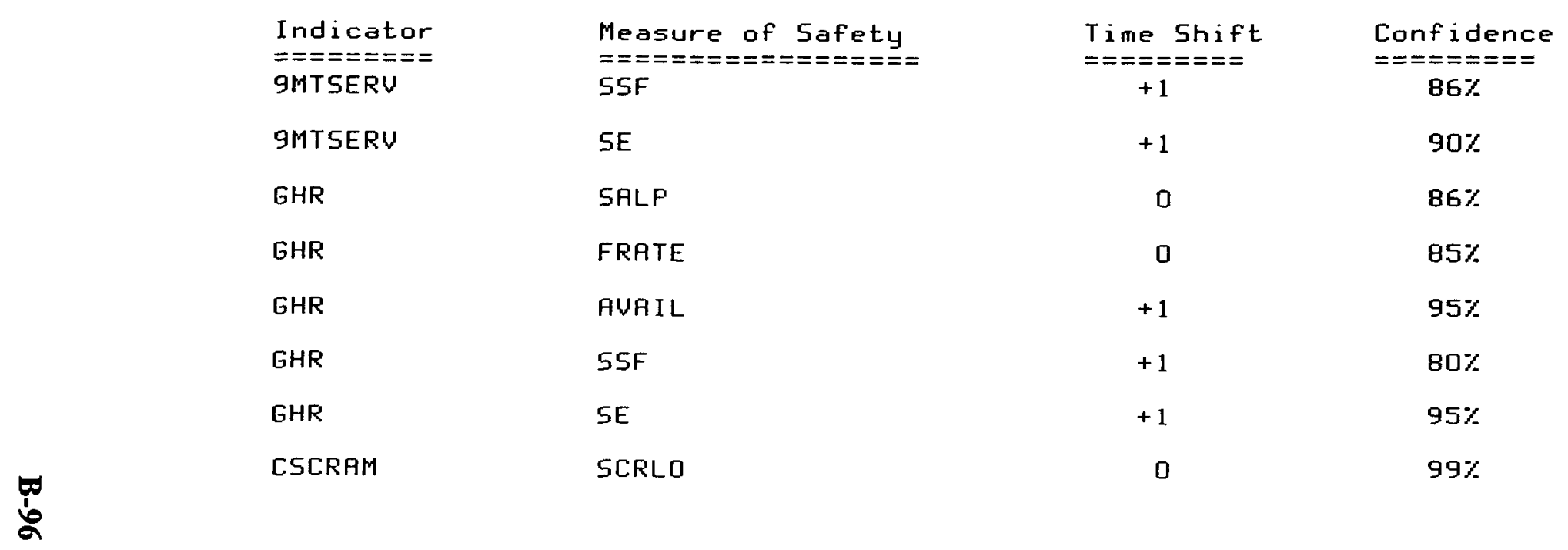



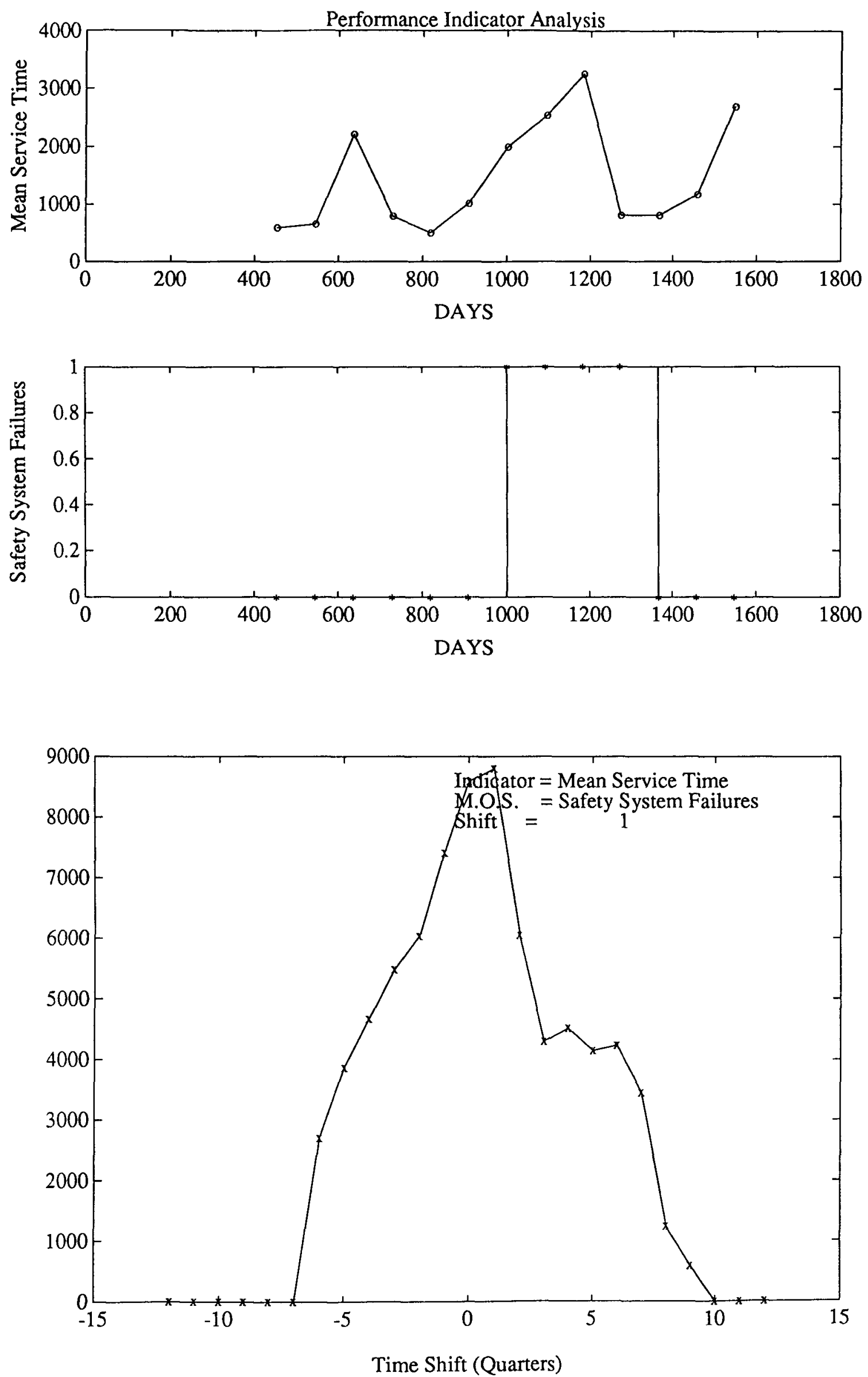

B-97 

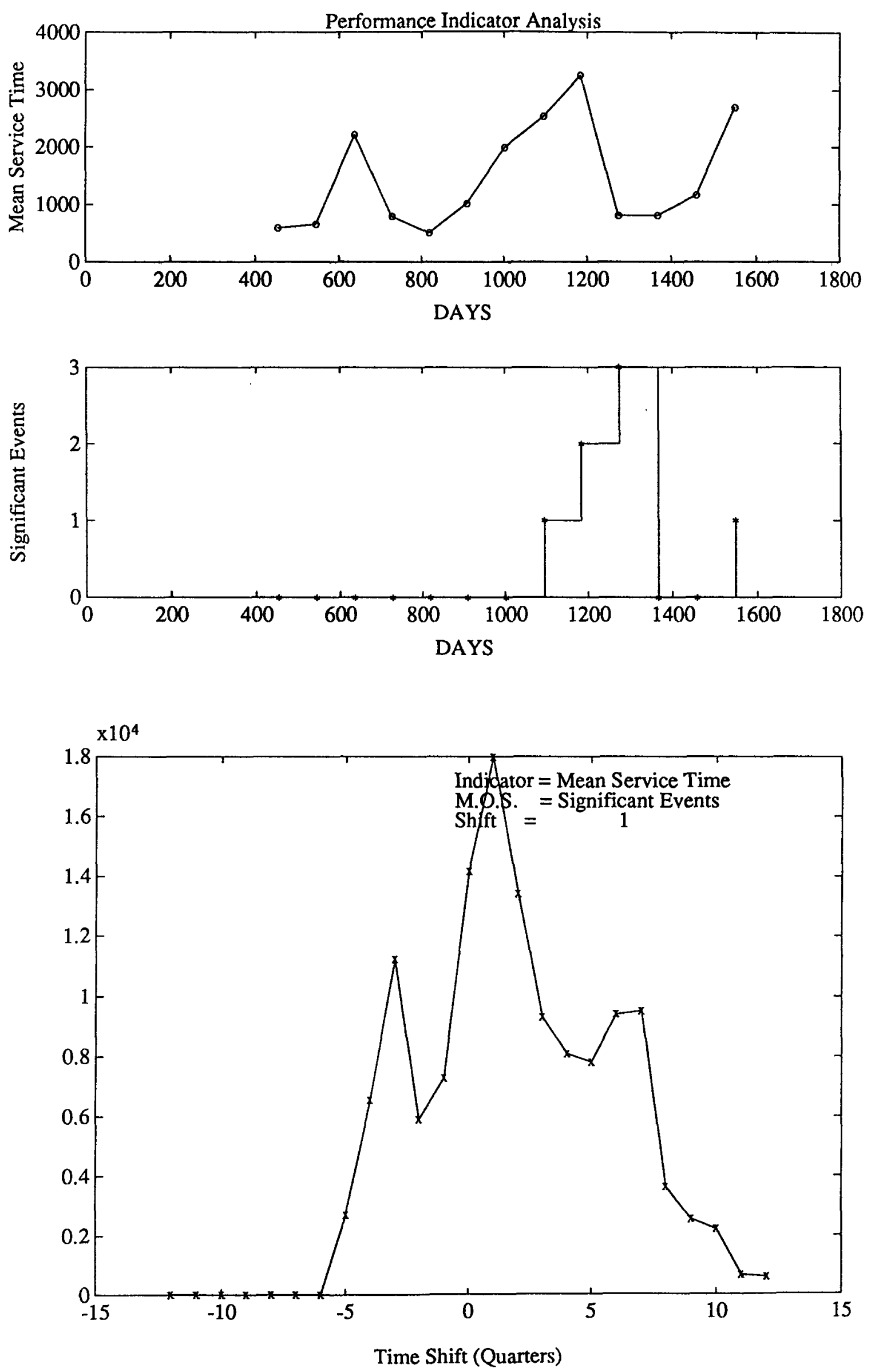

B-98 

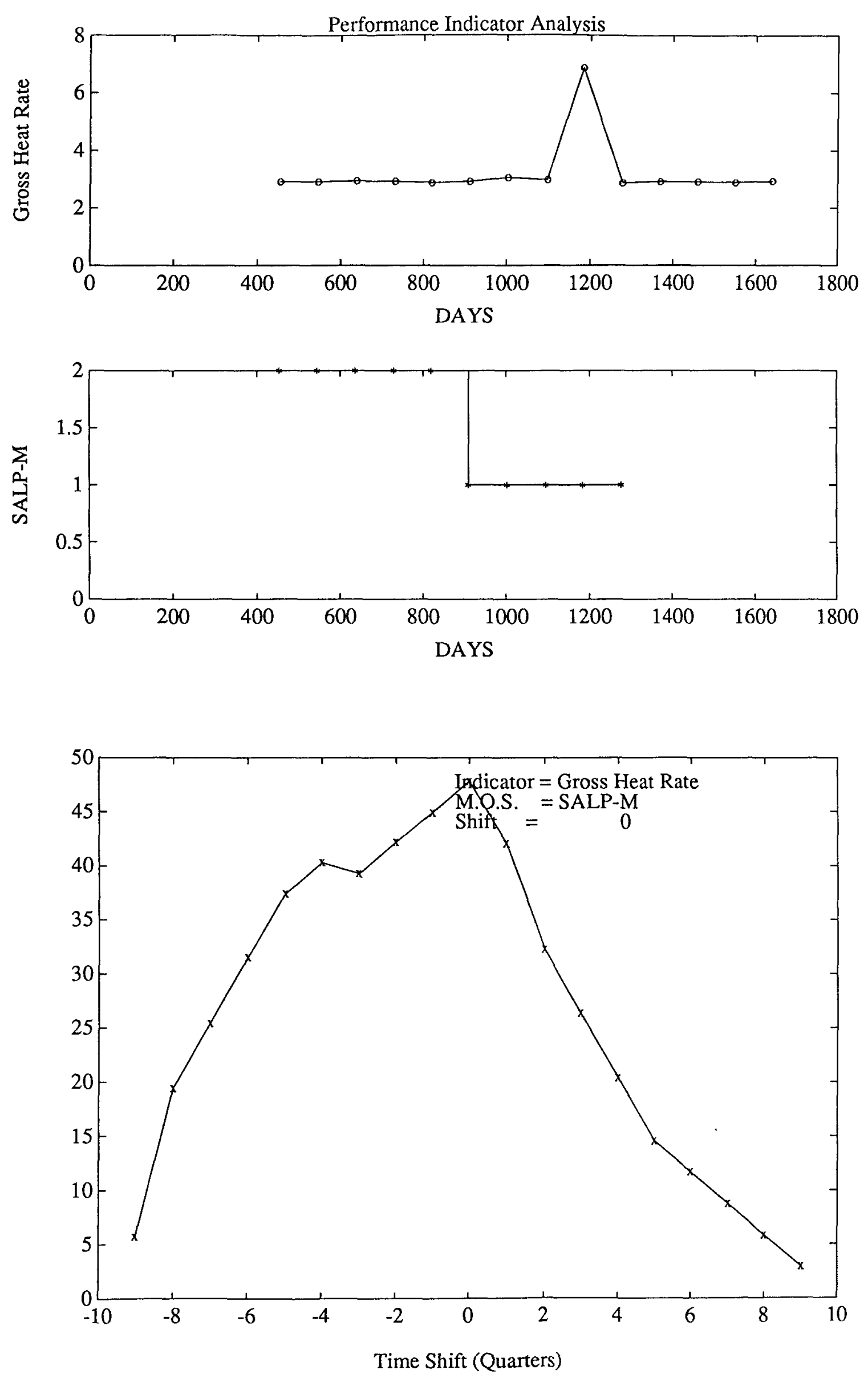

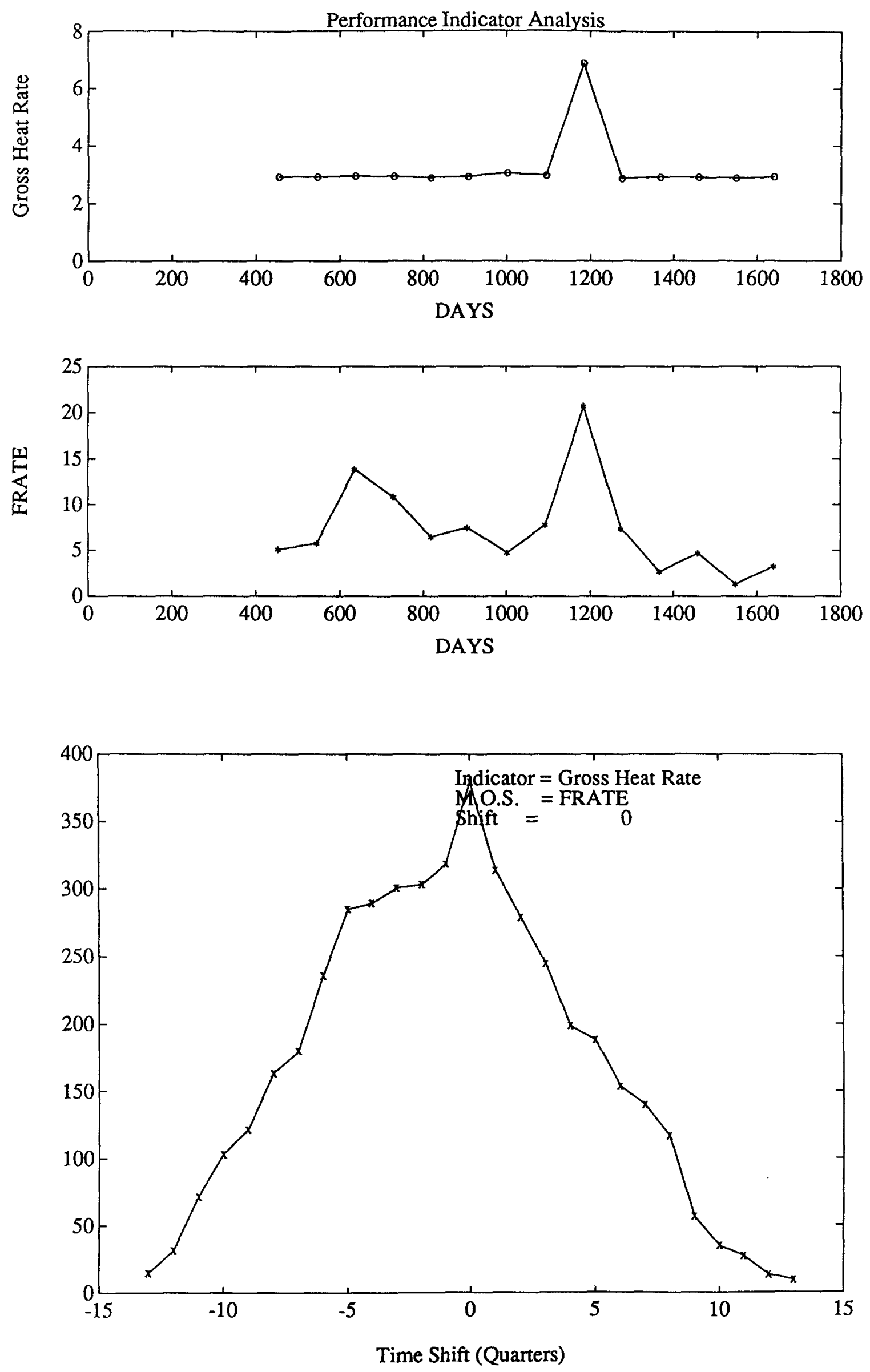

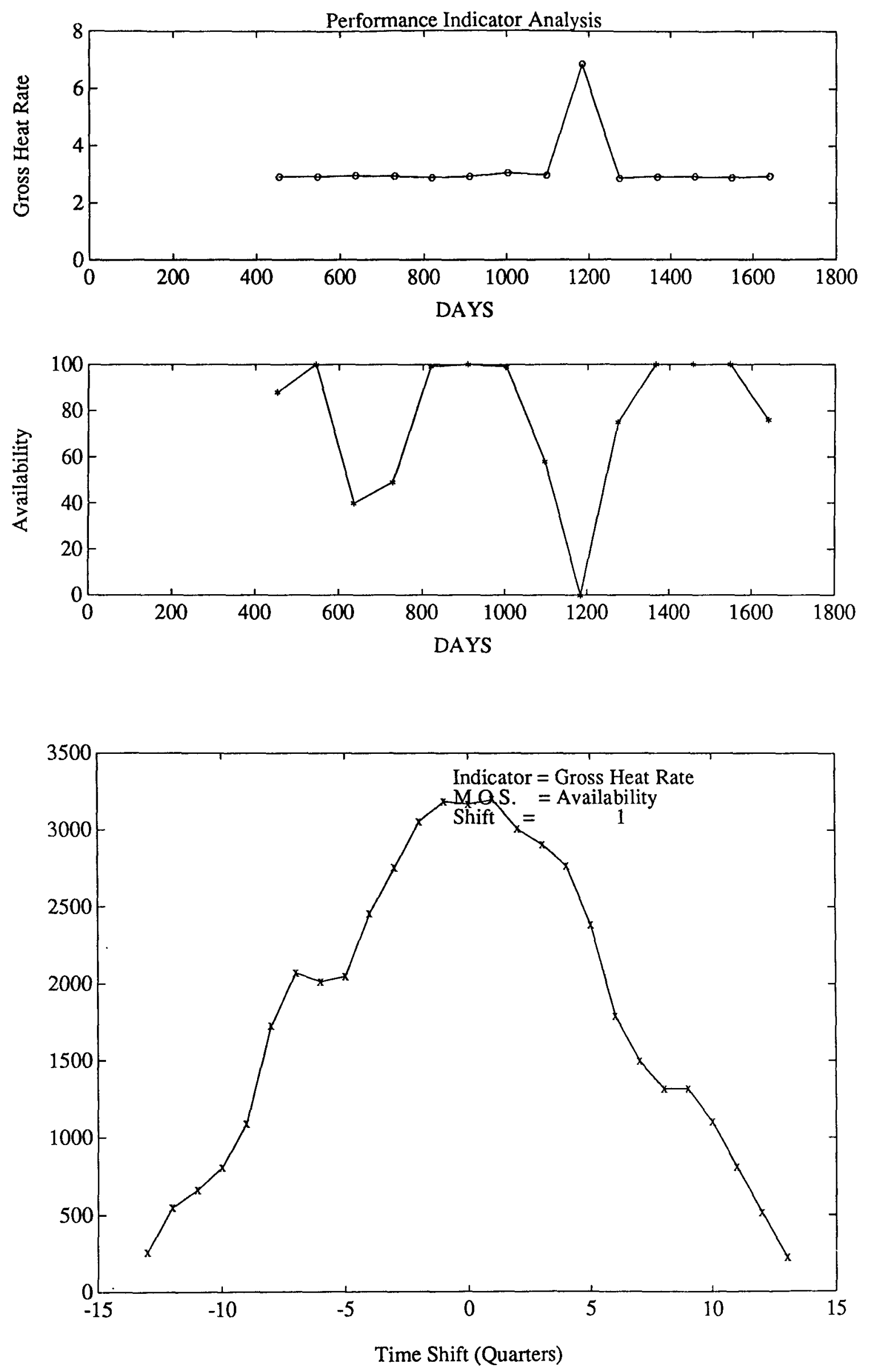

B-101 

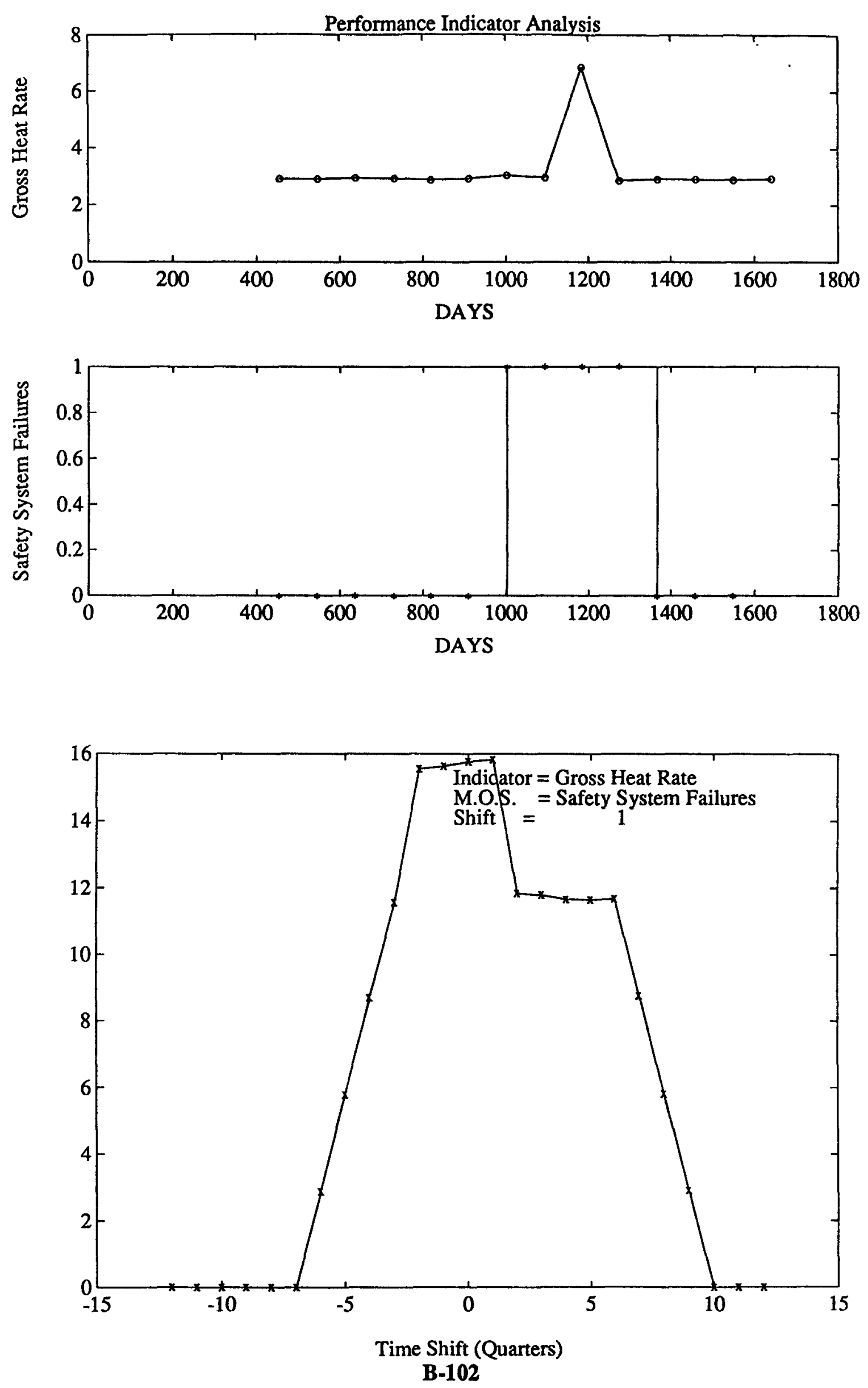

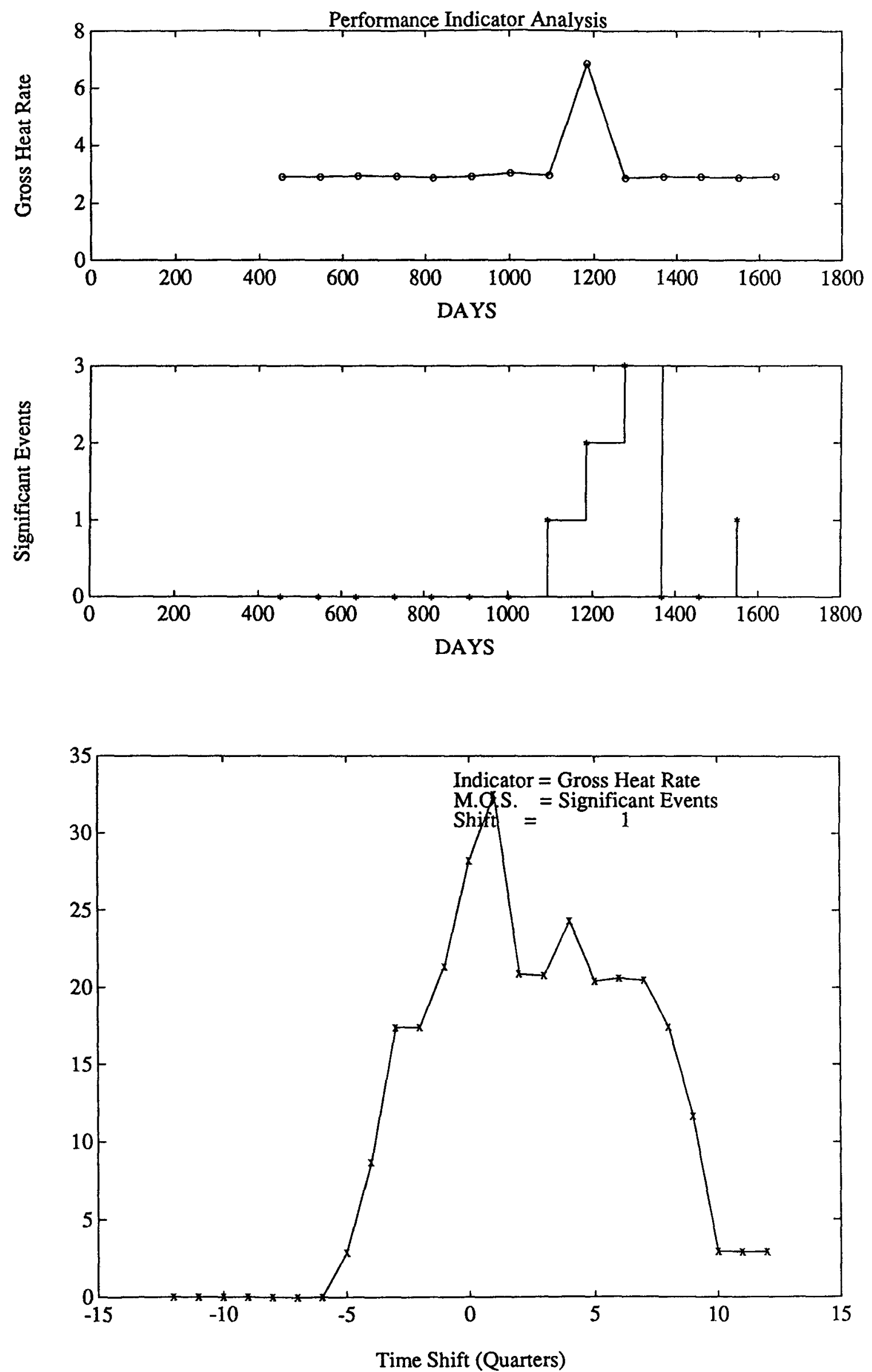

B-103 

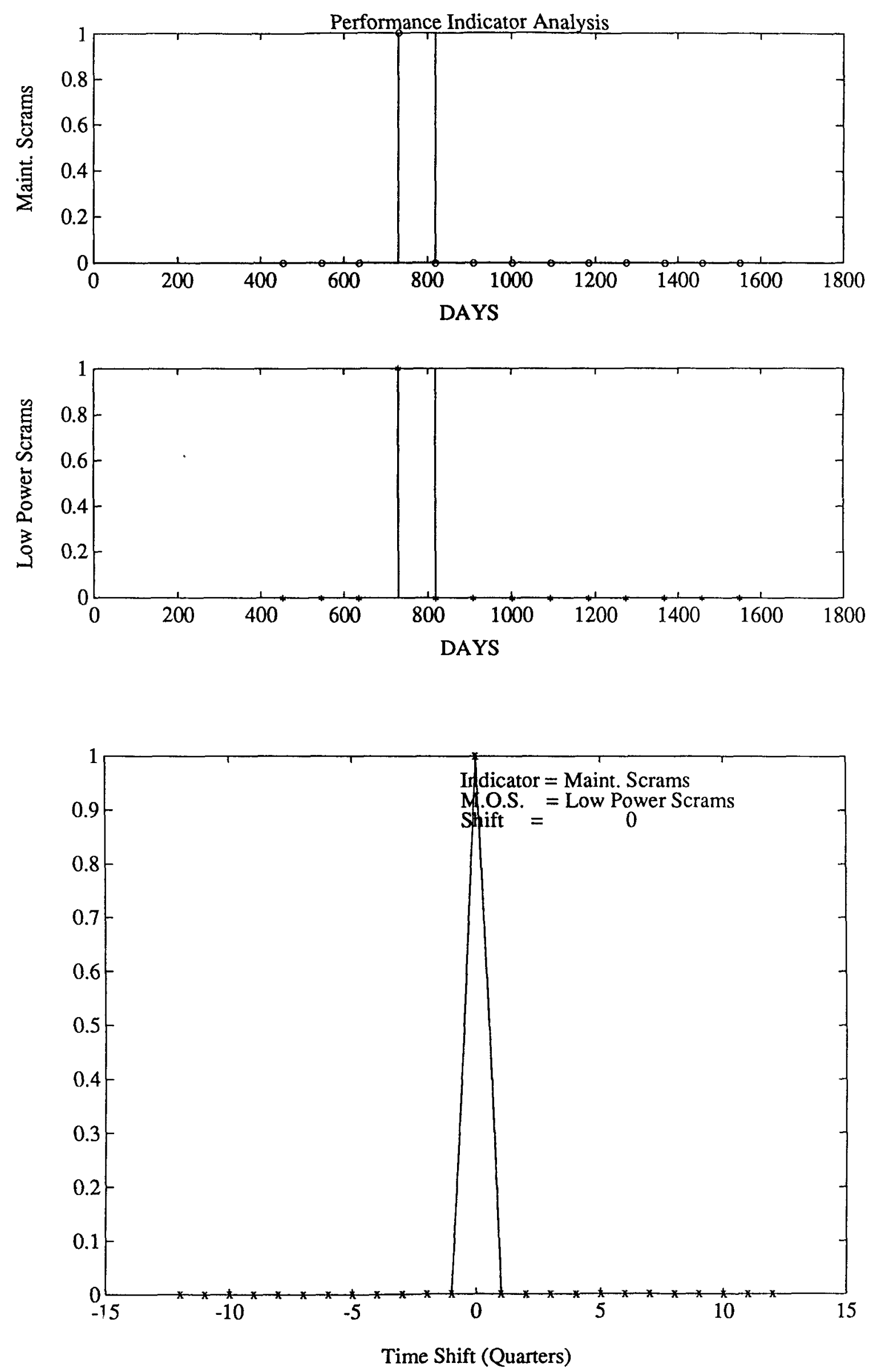
Attachment 1, Signal Processing Analysis of

Daily Power Loss Indicator

Att-1-1 


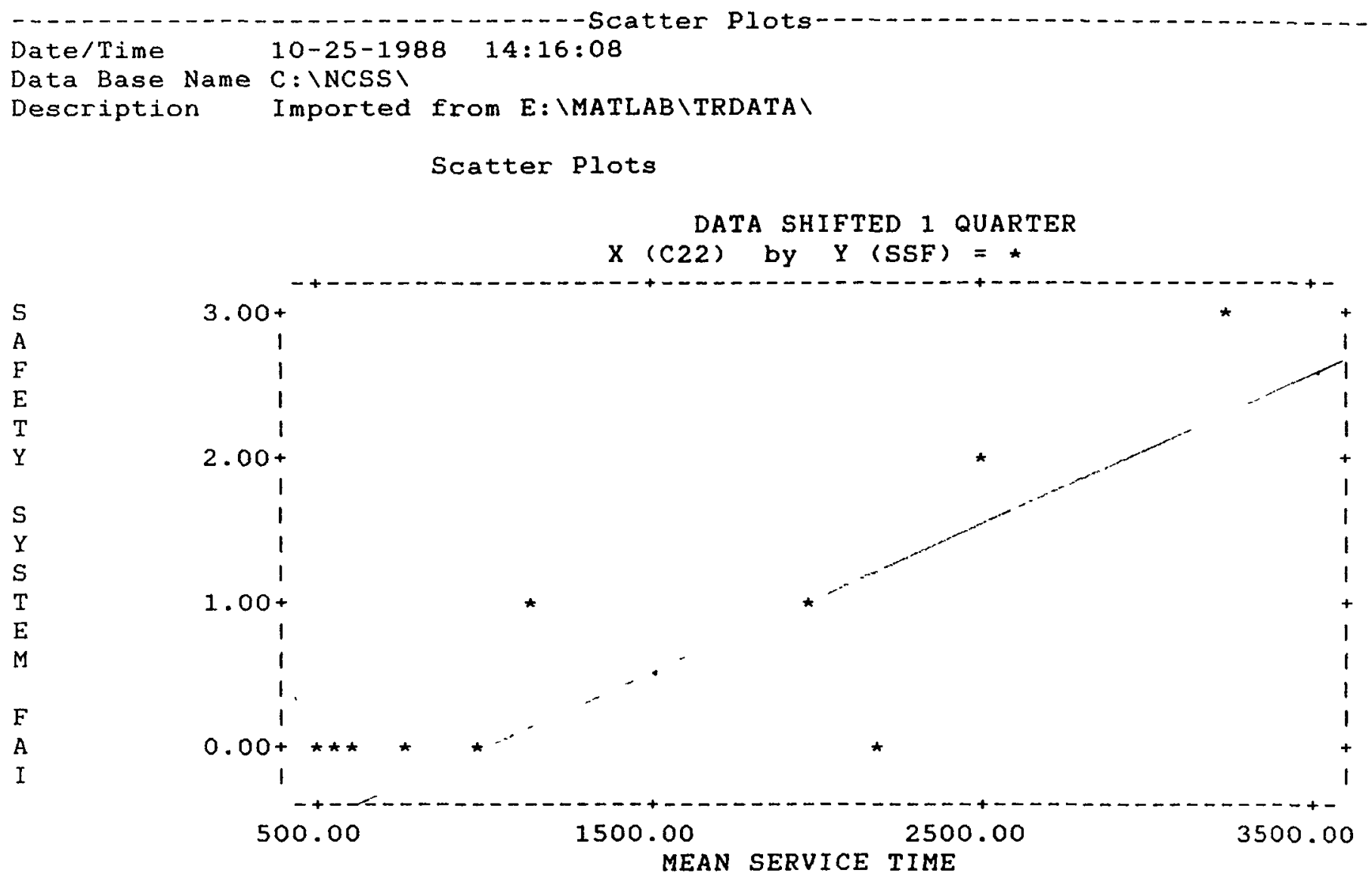

1.-w Accounting Report

$\begin{array}{lllrrr}\text { Number of points below minimum } & (X): & 0 & (Y): & 0 \\ \text { Number of points above maximum } & (X): & 0 & (Y): & 0 \\ \text { Number of points with missing values } & (X): & 1 & (Y): & 1 \\ \text { Number of points plotted } & & : & 12 & & \end{array}$



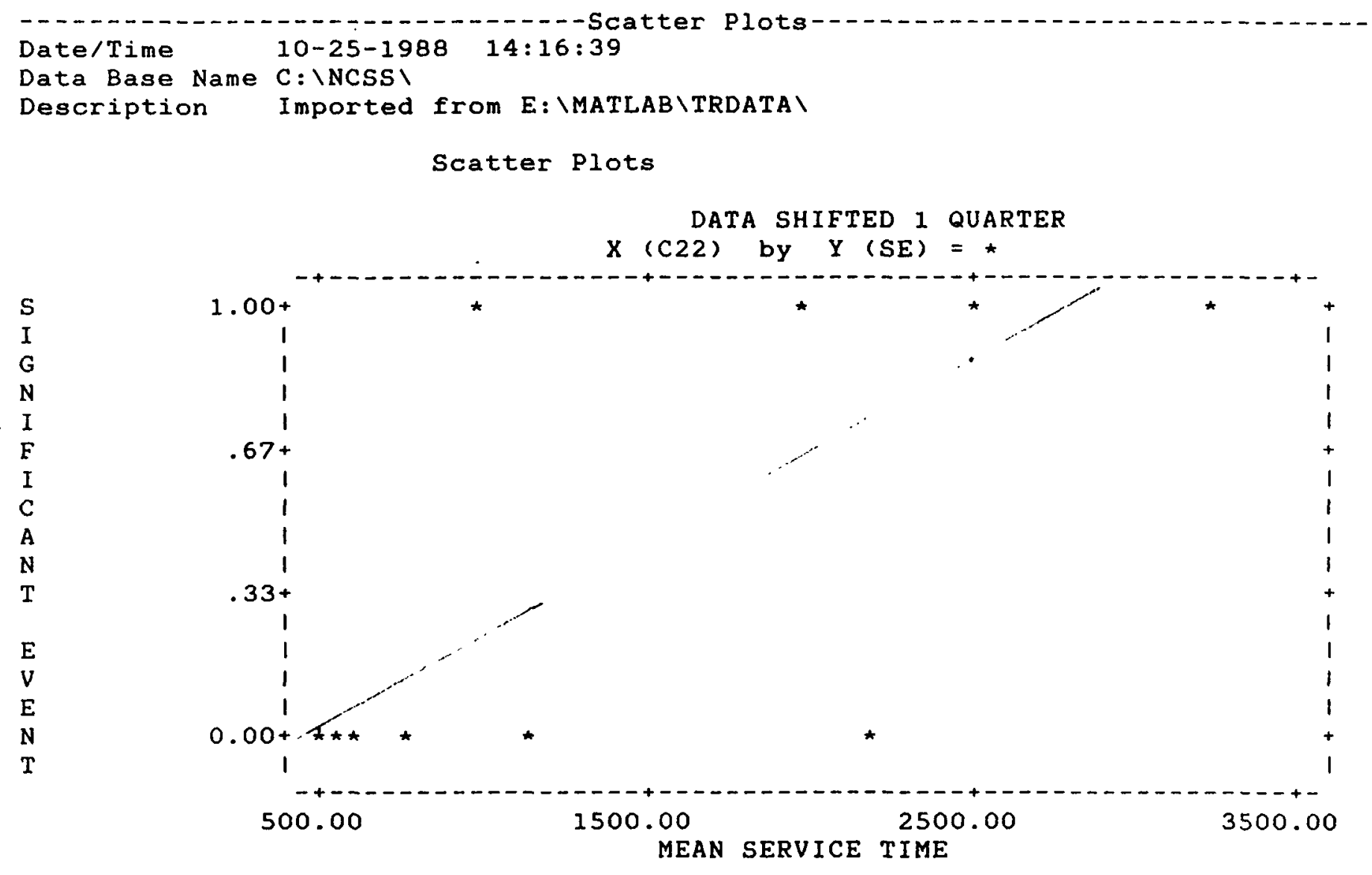

nuw Accounting Report

$\begin{array}{llrrrr}\text { Number of points below minimum } & (X): & 0 & (Y): & 0 \\ \text { Number of points above maximum } & (X): & 0 & (Y): & 0 \\ \text { Number of points with missing values } & (X): & 1 & (Y): & 1 \\ \text { Number of points plotted } & & : & 12 & & \end{array}$




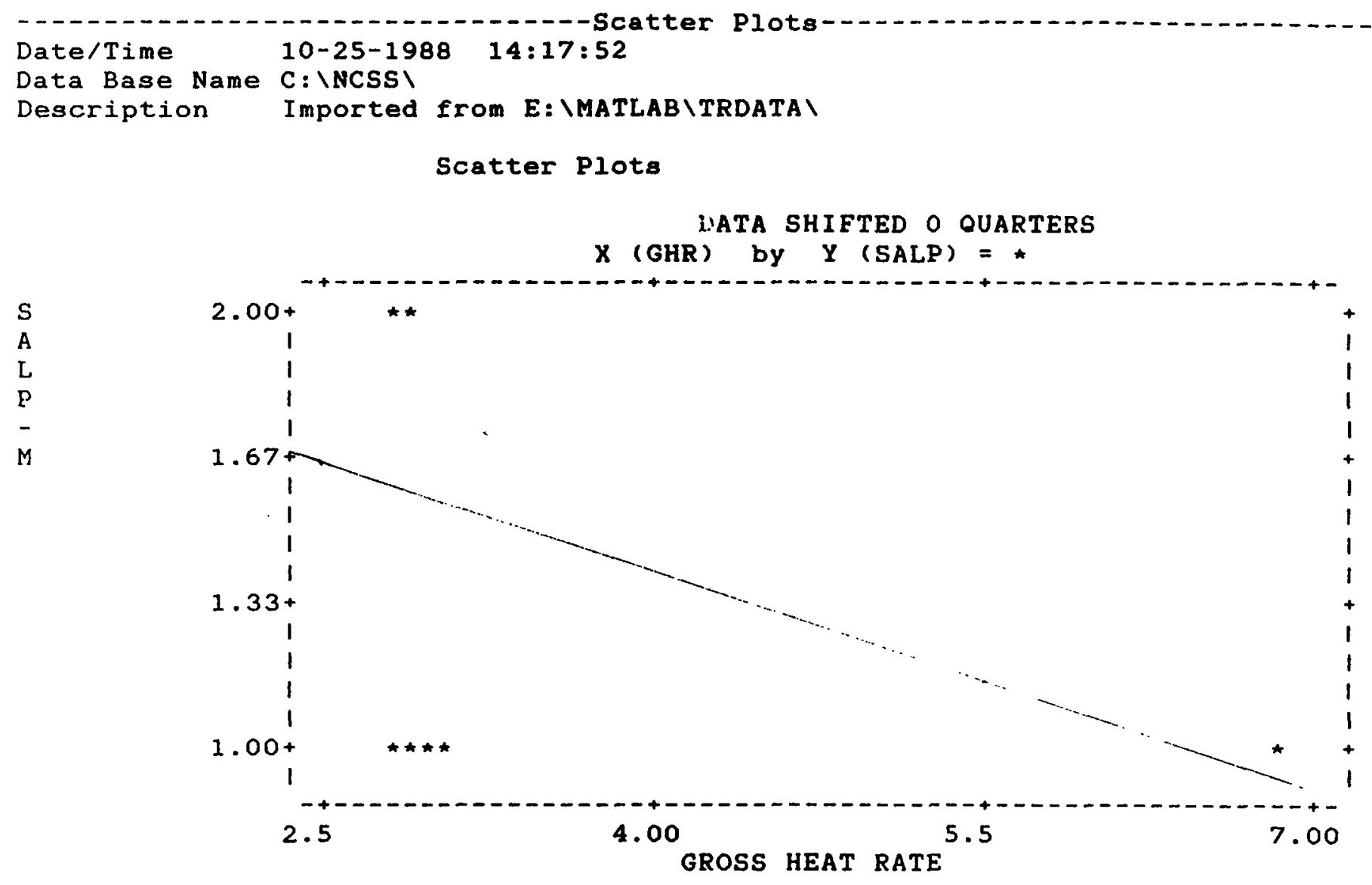

h * Accounting Report

$\begin{array}{lllllll}\text { Number of points below minimum } & (X): & 0 & (Y): & 0 \\ \text { Number of points above maximum } & (X): & 0 & (Y): & 0 \\ \text { Number of points with misging values } & (X): & 0 & (Y): & 4 \\ \text { Number of points plotted } & & : & 10 & & & \end{array}$




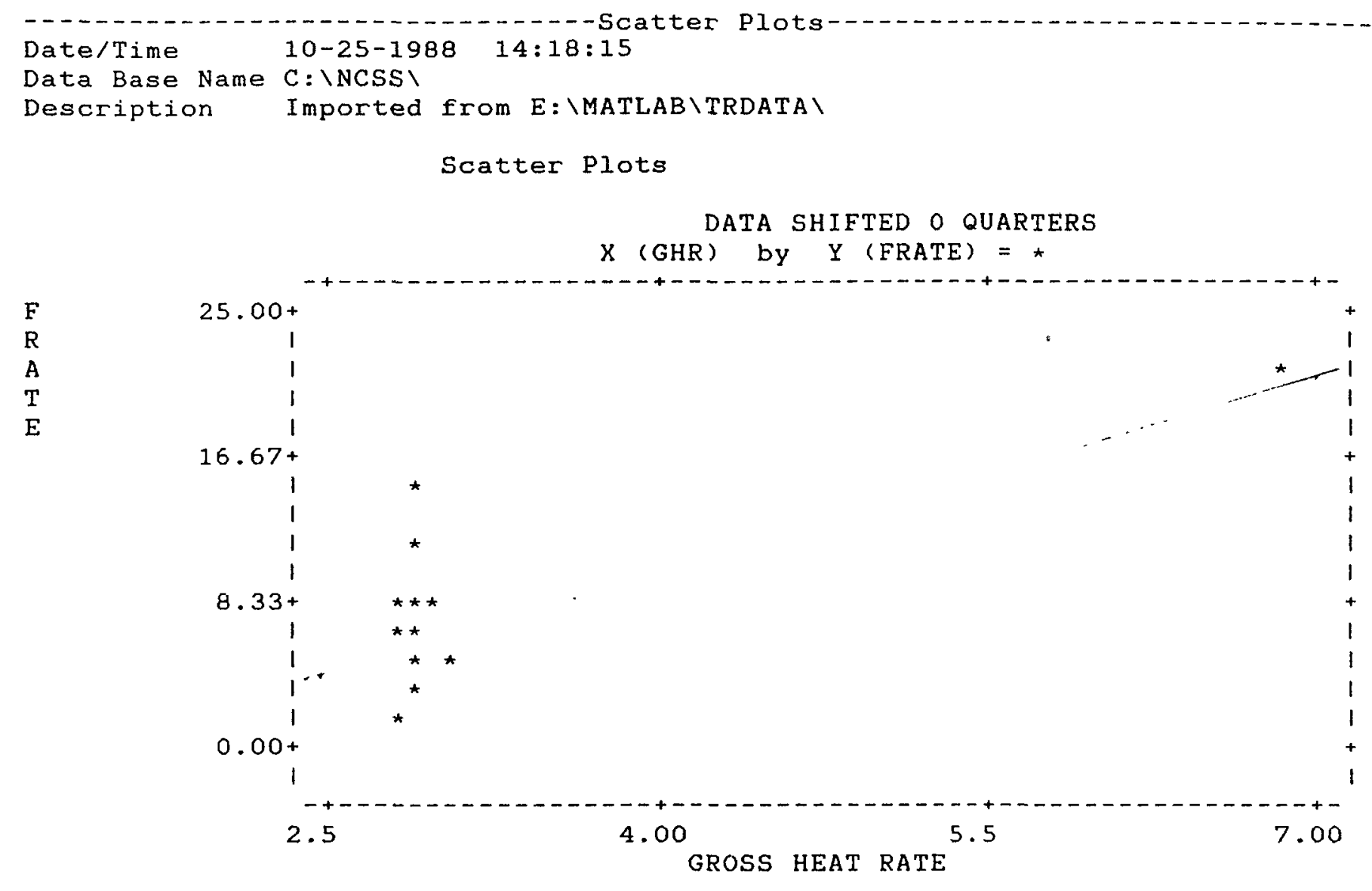

1.- W Accounting Report

$\begin{array}{lllrlll}\text { Number of points below minimum } & (X): & 0 & (Y): & 0 \\ \text { Number of points above maximum } & (X): & 0 & (Y): & 0 \\ \text { Number of points with missing values } & (X): & 0 & (Y): & 0 \\ \text { Number of points plotted } & & : & 14 & & & \end{array}$




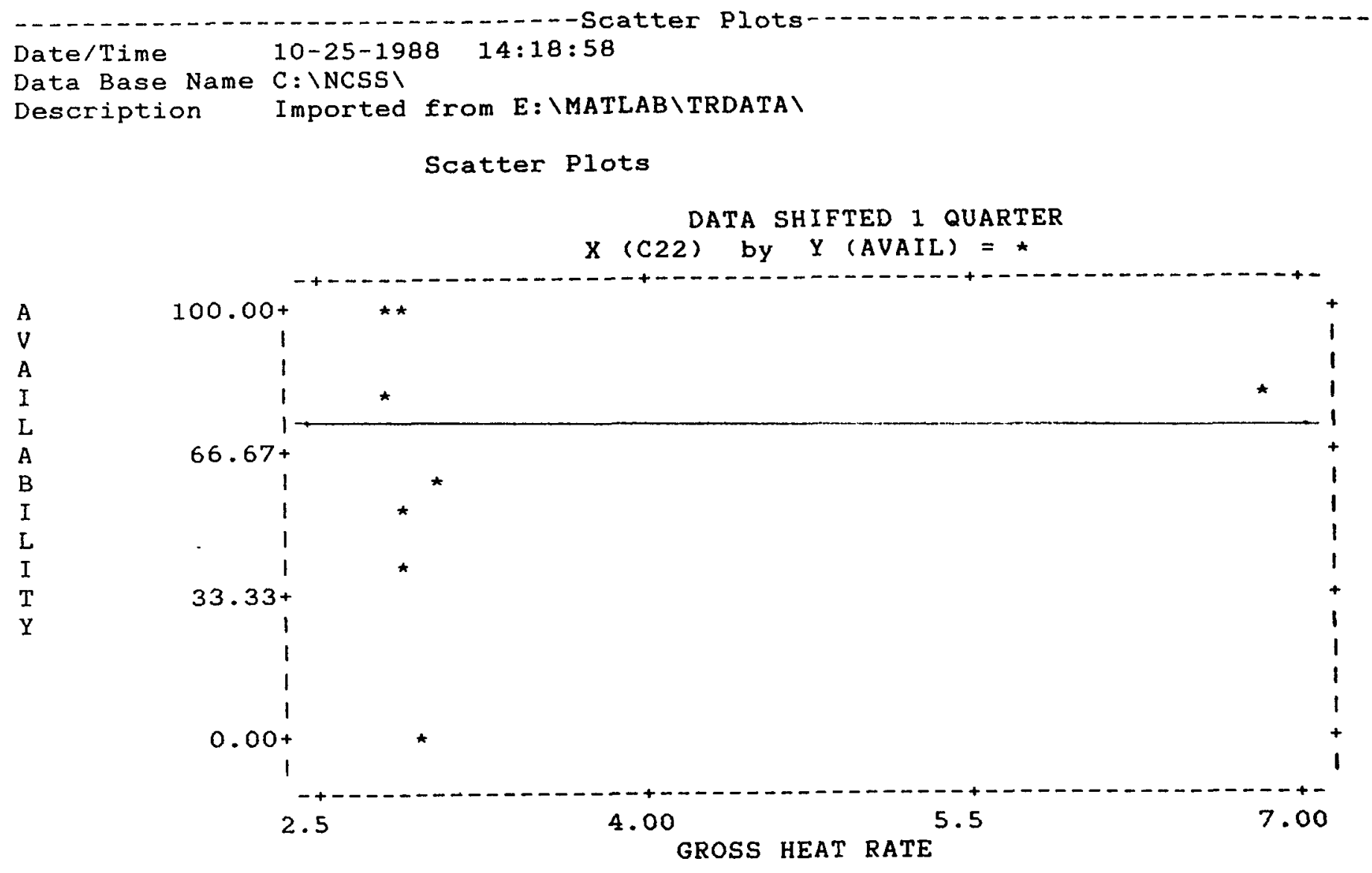

1.-w Accounting Report

$\begin{array}{lllllll}\text { Number of points below minimum } & (X): & 0 & (Y): & 0 \\ \text { Number of points above maximum } & (X): & 0 & (Y): & 0 \\ \text { Number of points with missing values }(X): & 1 & (Y): & 0 \\ \text { Number of points plotted } & & : & 13 & & & \end{array}$




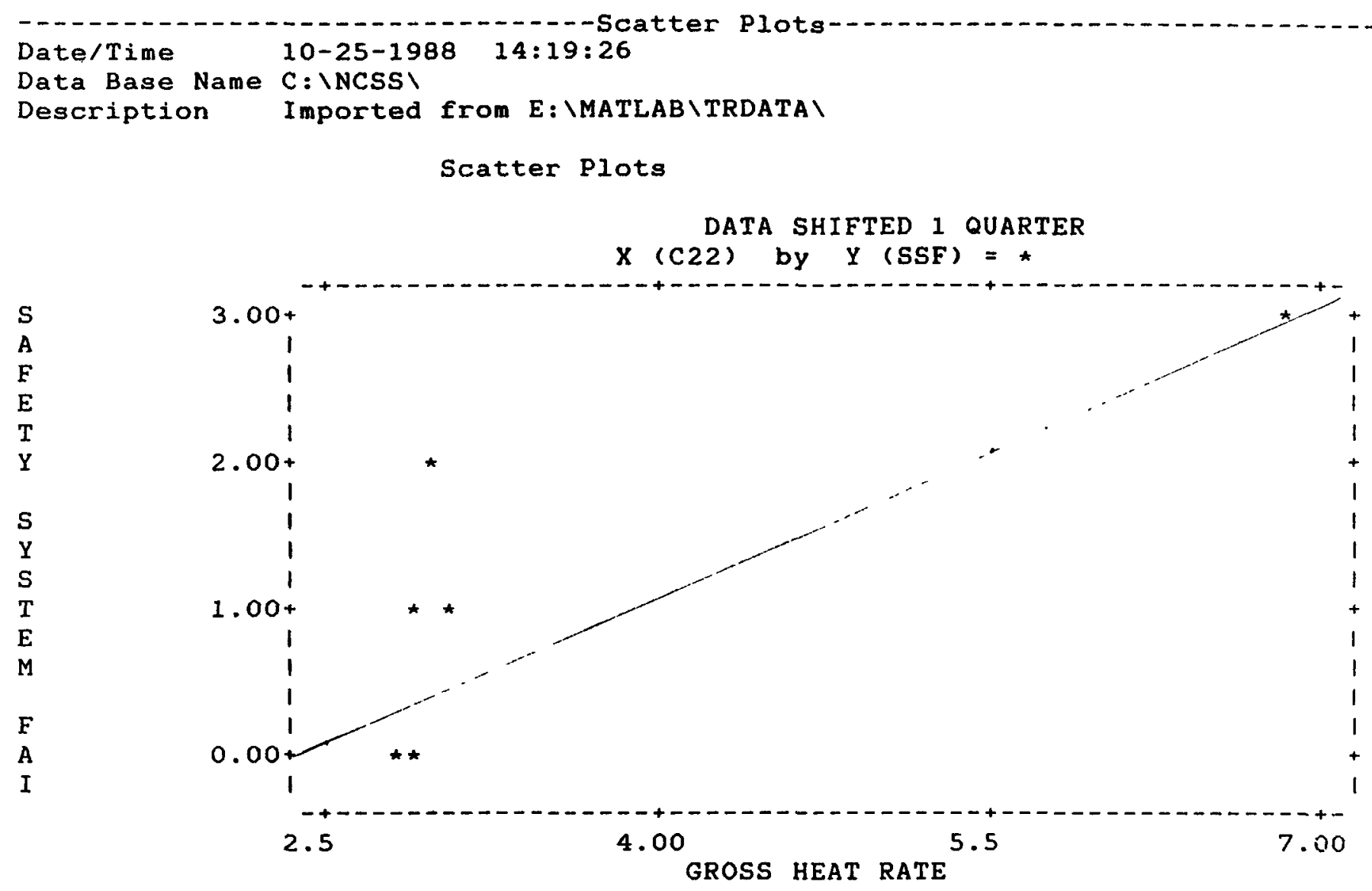

1.-W Accounting Report

Number of points below minimum $\quad(X):=0$

Number of points above maximum $\quad(X):=0$

Number of points with missing values $(X):=11$ ( $Y$ ) : 1 

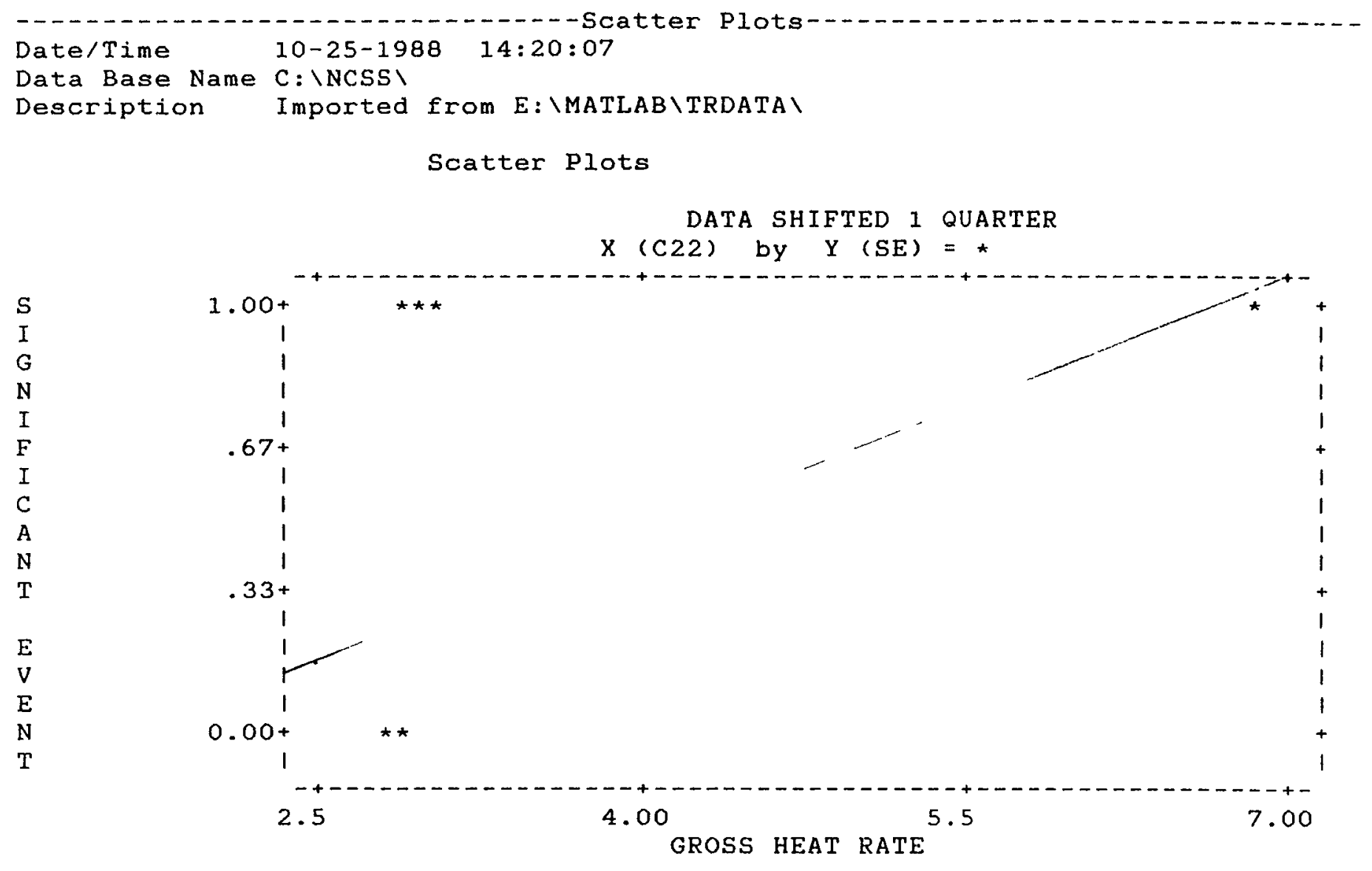

* Accounting Report

Number of points below minimum

Number of points above maximum

$(X): 0 \quad(Y): 0$

Number of points with missing values

(X) :

Number of points plotted

$(X):=1$

(Y) : $: 0$

(Y) : 1 


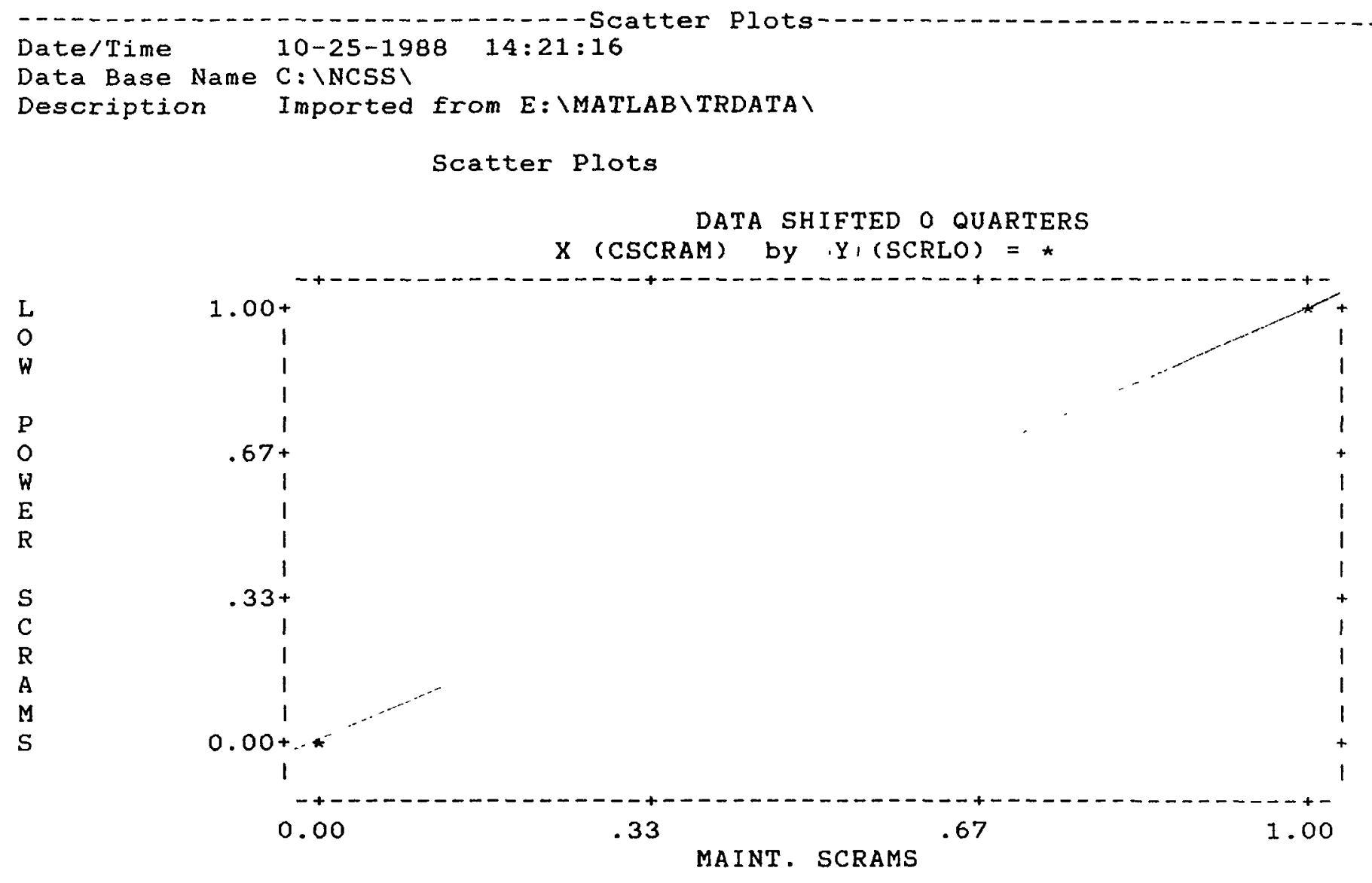

w Accounting Report

$\begin{array}{lllllll}\text { Number of points below minimum } & (X): & 0 & (Y): & 0 \\ \text { Number of points above maximum } & (X): & 0 & (Y): & 0 \\ \text { Number of points with missing values } & (X): & 0 & (Y): & 0 \\ \text { Number of points plotted } & & : & 13 & & & \end{array}$




\section{POTENTIALPEREORMANCE INDICATORS RELATED TO THERMALEFFICIENCY}

\subsection{DEVELOPMENT OE A THERMAL-EFFICIENCY-RELATED COMPREHENSIVE MAINTENANCE INDICATOR}

\subsection{Gross Heat Rate or Thermal Efficiency Indicators}

Previous work on the maintenance performance indicators program led to gross heat rate being proposed as a promising leading indicator of nuclear plant safety trends. Gross heat rate, the electrical generation industry's conventional way of expressing the overall thermal efficiency of a thermal power plant, is defined as the ratio of the total thermal energy input to the plant, to its total electrical energy output during some interval of time. For nuclear plants, the thermal input is the heat energy produced by the reactor core. (Since heat rate is the algebraic reciprocal of thermal efficiency if consistent units are used, the two quantities are equivalent, and the terms will be used interchangeably below.)

The rationale for thermal efficiency as a trending indicator of nuclear plant safety is that over the long term, both safety and thermal efficiency depend on high-quality plant maintenance (broadly defined to include maintenance, operations, engineering, management,and all other plant functions which help to preserve operability), so trends in plant safety should follow trends in thermal efficiency. In fact, thermal efficiency should be more sensitive to maintenance quality than safety, for two reasons. First, the non-safety-related portion of the plant continuously generates information on its own status during operation, while safety depends on either passive features (e.g., containment) or standby systems whose status is only known intermittently. Therefore, declining efficiency will presumably be noticed and corrected first. Second, for business reasons the licensee presumably places at least as much priority on thermal efficiency as safety. In other words, long-term neglect of the maintenance needed for the plant to fulfill its basic mission -- generating electrical energy efficiently .- may indicate even greater neglect of the nuclear safety functions which are secondary to the plant's reason for being (however critical they may be from the NRC perspective). It is also a well established fact that reactor SCRAMS and safety system challanges are mainly attributable to BOP system and component failures. Thus it was suggested that gross heat rate would be a comprehensive indicator of maintenance performance in its broadest sense.

Att-1-10 
However, gross heat rate or thermal efficiency per se, while suggestive, has three deficiencies as an overall indicator of maintenance quality. First, heat rate is essentially meaningless when the plant is shut down. Second, a power plant has its maximum thermal efficiency at its design load, so extended off-design loading for reasons external to the plant (e.g., transmission grid problems or low power demand) could produce a misleading decline in efficiency. Third, the NRC is not currently collecting the data required to calculate heat rate. The ideal indicator would reflect thermal efficiency, deal realistically with shutdowns and load fluctuations, and preferably be based on data the NRC is already receiving in licensees' monthly operating reports.

\subsection{Average Daily Power Level as an Indicator}

Monthly operating reports contain only one piece of information which relates to thermal efficiency and is reported on a daily basis so it can be trended over the short term. This is the average daily power level (ADP), defined as the quotient of total electrical energy output in MWh during the 24-hour period divided by 24 hours. Unfortunately, ADP is no more an ideal maintenance indicator than heat rate, because non-maintenance factors such as planned refueling outages and load changes can affect it. However, ADP has the advantage of tracking another maintenance-related parameter in addition to thermal efficiency, the incidence of forced outages of major equipment in the plant, because virtually any major equipment failure precipitates at least a power reduction if not a shutdown. Therefore, ADP -- or some parameter closely related to it -- should be an integrated indicator of both plant thermal efficiency and the availability of major equipment, and hence a more sensitive indicator of trends in maintenance quality than thermal efficiency alone. SAIC therefore concentrated on attempting to extract meaningful maintenance quality indications from patterns discernible in ADP data.

\section{3 "Daily Power Loss"}

For several reasons which will be discussed below, SAIC found it more convenient to analyze a parameter which we call "daily power loss" (DPL) rather than working directly with ADP. Daily power loss is a normalized, inverse measure of average daily power level defined as follows:

$D P L_{n}=\frac{M D C-A D P_{n}}{M D C}-A D P_{\text {aye }}$ 
$A D P_{\text {ave }}=\frac{1}{(N)(M D C)} \sum_{n=0}^{N} M D C-A D P_{n}$

where DPL is the daily power loss for day $n$,

MDCn is the maximum dependable net capacity of the plant as given in the monthly operating report,

$A D P_{n}$ is the average daily power level for day $n$,

$A D P$ ave is the average of all ADPs for $N$ days, and

$N$ is the number of days in the interval being analyzed.

DPL is equivalent to ADP in terms of information content, and has the following three advantages. First, higher DPL relates to poorer performance, which is consistent with the sense of the other indicators being considered. Second, the normalization by plant MDC eliminates two irrelevant plant-specific factors, plant capacity and design heat rate, so DPL is consistent from plant to plant. Third, the DPL waveform is symmetrical about the horizontal axis, which reduces the computation load by making the coefficients of all even-numbered terms in the Fourier series representation of the waveform zero. (See below for a discussion of Fourier analysis.)

\subsection{ANALYSIS AND EVALUATION OF DAILY POWER LOSS AS A MAINTENANCE PERFORMANCE INDICATOR}

\subsection{Data Acquisition and Units Covered}

The first step in the attempt to extract maintenance performance trends from DPL data comprised data acquisition. SAIC obtained 1985-1987 monthly operating reports for eight units, selected to obtain a representative sample of plant designs, nuclear steam supply system (NSSS) suppliers, and "good" and "bad" plants. The table below lists the units considered in the study, with their reactor types, NSSS suppliers, and architect/engineer firms (A/Es). 
IABLE 1. UNITS INCLUDED IN STUDY

\begin{tabular}{|c|c|c|c|}
\hline Plant & $\begin{array}{l}\text { Reactor } \\
\text { Type }\end{array}$ & $\begin{array}{l}\text { NSSS } \\
\text { Supplier }\end{array}$ & $A / E$ \\
\hline$=====$ & $=====$ & 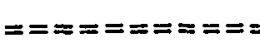 & $====-===x==$ \\
\hline $\mathbf{S}$ & PWR & $B \& W$ & Bechtel \\
\hline$T$ & BWR & GE & UE\&C \\
\hline $\mathbf{U}$ & PWR & $B \& W$ & Bechtel \\
\hline $\mathbf{V}$ & PWA & Westinghouse & UE\&C \\
\hline$W$ & BWR & GE & S\&L \\
\hline$X$ & PWR & $B \& W$ & $\begin{array}{l}\text { Duke Powerl } \\
\text { Bechtel }\end{array}$ \\
\hline $\mathbf{Y}$ & PWR & CE & Bechtel \\
\hline$Z$ & PWA & Westinghouse & Bechtel \\
\hline
\end{tabular}

\subsection{Preliminary Analytical Approach}

\subsubsection{Daily Power Loss Versus Time}

Plant $S$ was selected as the pathfinder unit for the analysis. The first step consisted of plotting the Daily Power Loss versus time for Plant $S$ to determine if any patterns related to maintenance quality could be discerned. Figures 1-3 show these plots for 1985 through 1987 for Plant S, together with notes identifying all of the incidents that precipitated changes in power level that were identifiable from monthly operating reports and LERs. Apart from the long "flat" interval during the extended

\section{Att-1-13}




\section{Detailed Results for Plant S}

Att-1-14 


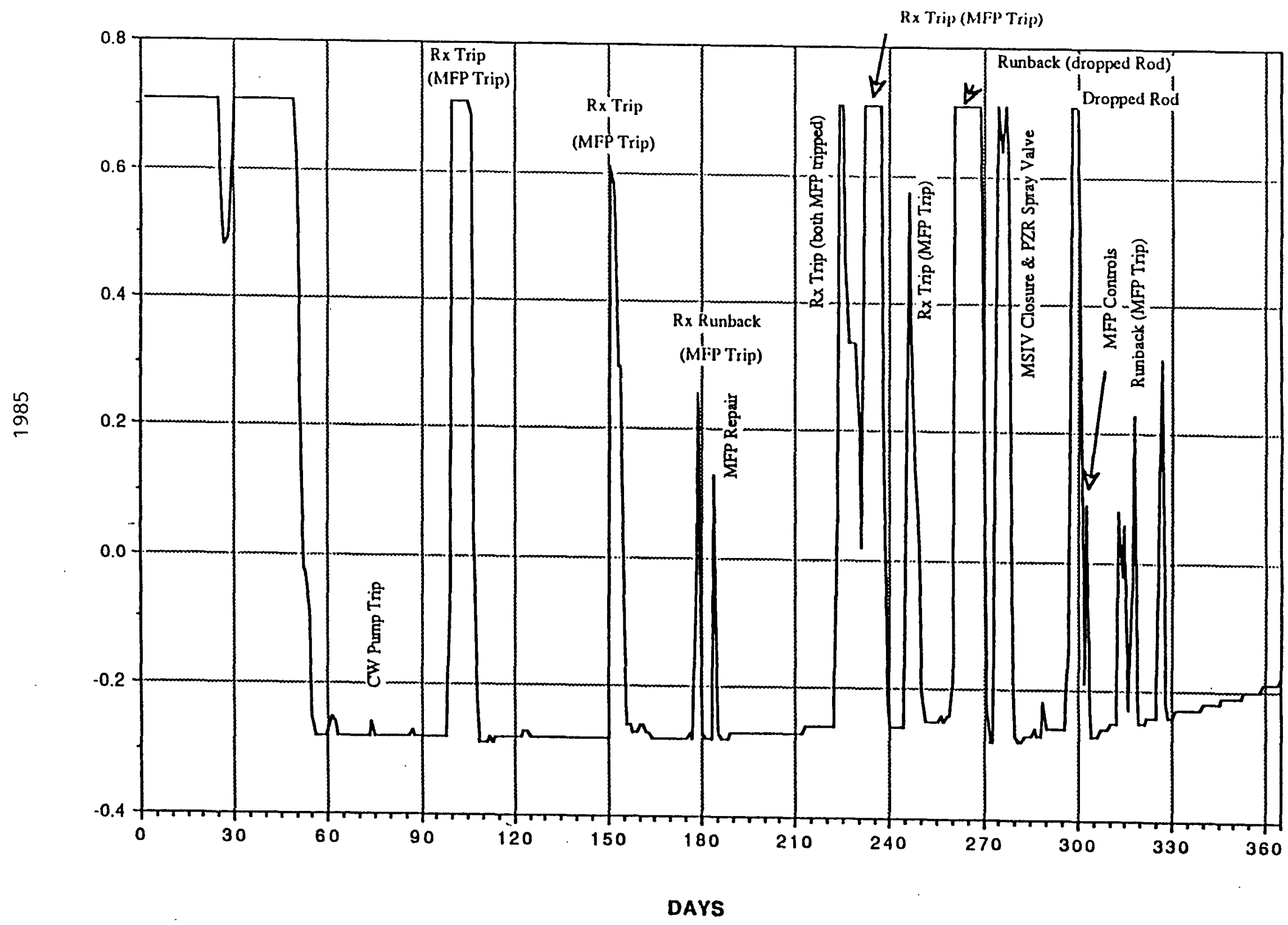

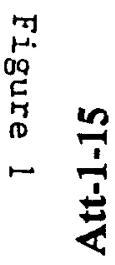




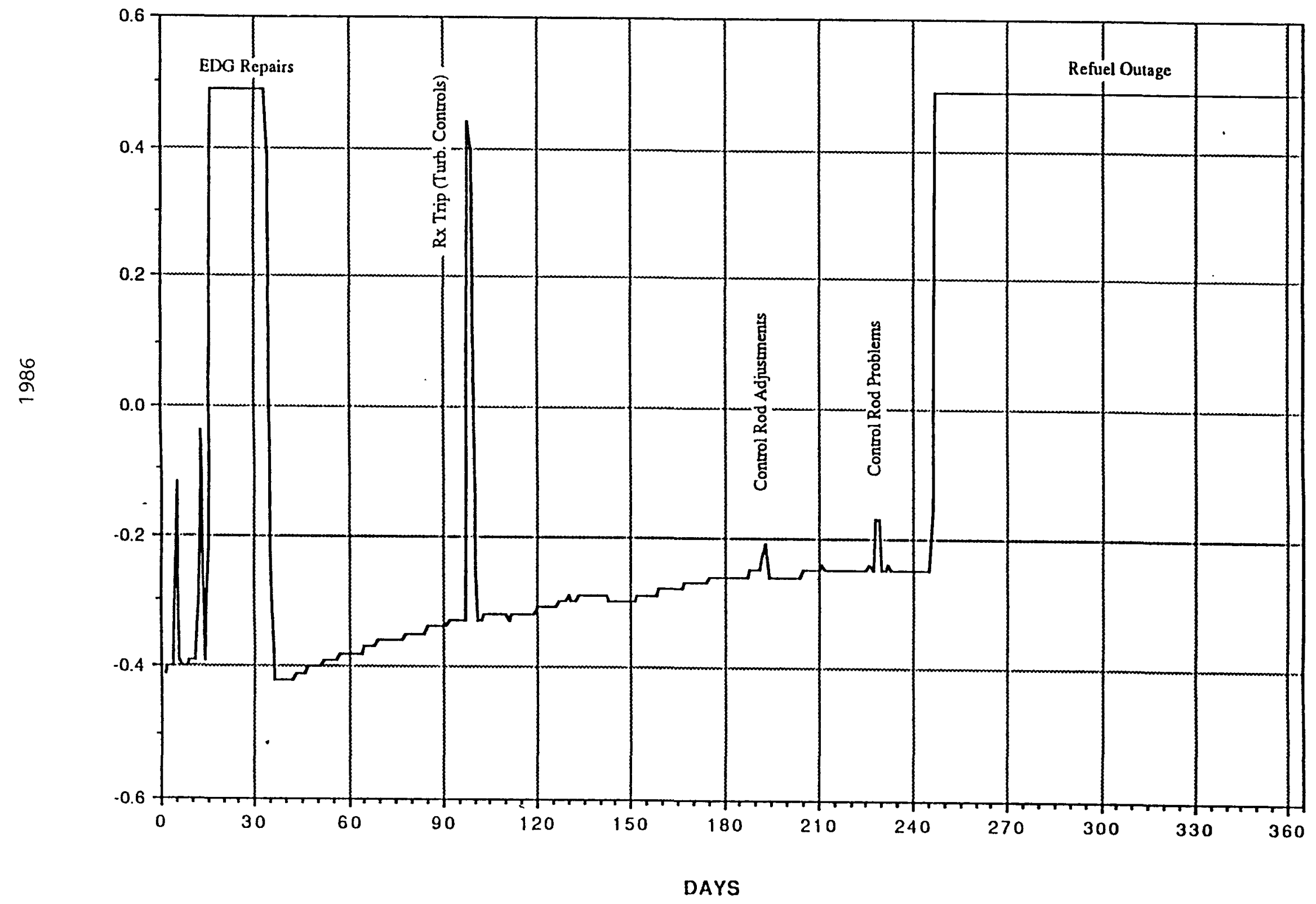

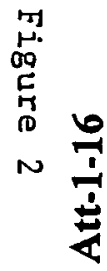




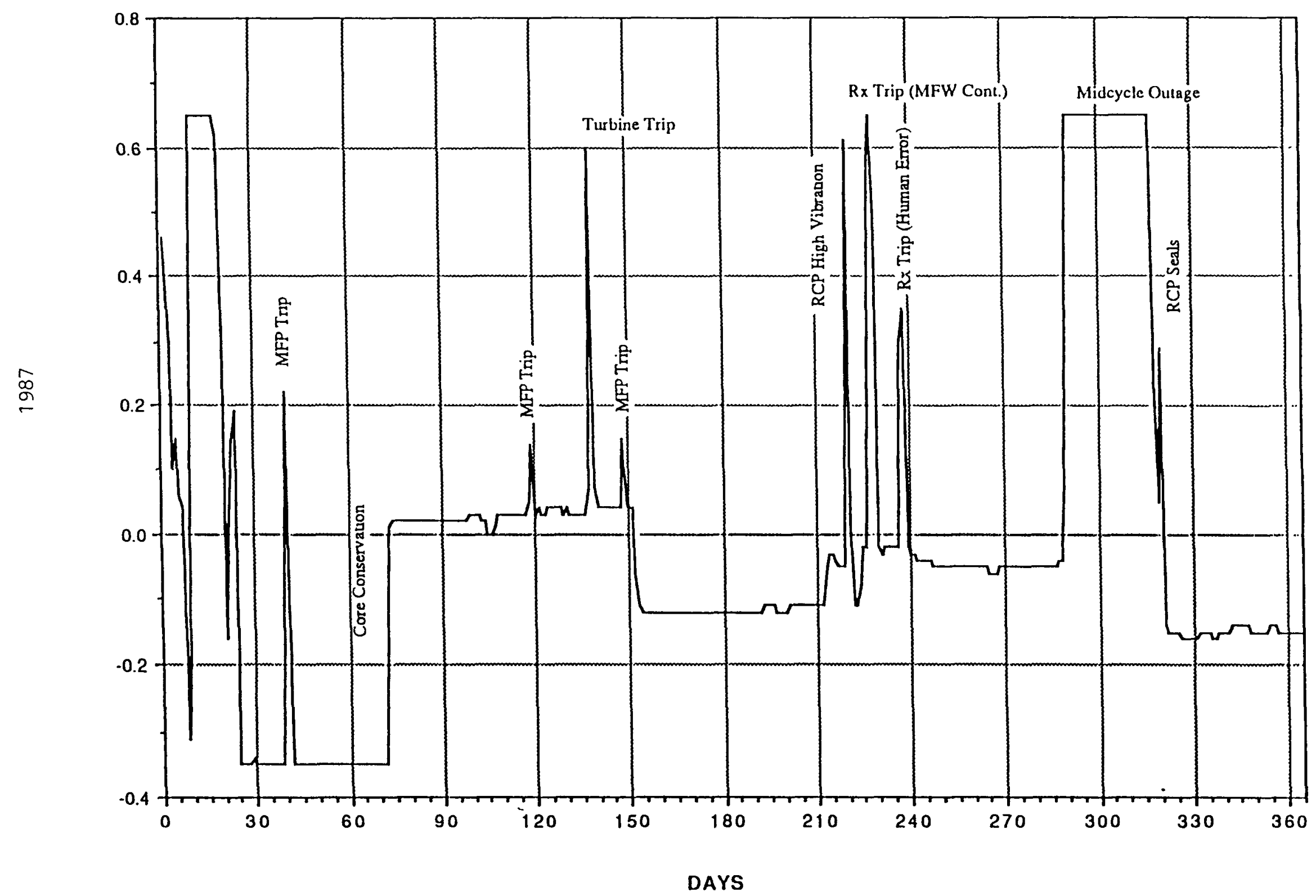

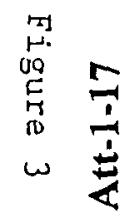


outage in 1986, no pattern is apparent. If maintenance-related patterns exist, they must be subtle, obscured by irrelevant information, or both. The same observation applies to the DPL data from the other seven plants, whose DPL-time plots appear in Appendix 1. The problem then is to process the data in order either to detect and amplify any patterns which actually exist or to eliminate the hypothesis that patterns do exist.

The concept underlying our current analytical approach is to look at the power plant as a "signal generator." The series of DPLs from the monthly operating reports can be considered as a set of equal-interval samples of a continuous time-varying signal which is susceptible to the signal-processing techniques developed to derive meaningful information from noisy signals in the communications field. The signal processing technique which is now under consideration is Fourier analysis.

\subsubsection{Review of Fourier Analysis Principles}

By way of review, Fourier analysis depends on the principle that any periodic mathematical function, no matter how complicated, is the sum of a possibly infinite series of sinusoidal terms. The simplest formulation of the Fourier series is as follows:

$$
f(t)=A_{0}+\sum_{n=1}^{\infty} A_{n} \sin \left(2 \pi F_{n} t+\theta_{n}\right)
$$

where $f(t)$ is the mathematical function representing the time-varying periodic signal to be decomposed,

$A_{n}$ is the amplitude (peak magnitude) of component $n$,

$F_{n}$ is the frequency of component $n$, and

$\theta_{n}$ is the phase angle of component $n$ (relative to an arbitrary reference).

That is, if a complicated signal can be represented by a periodic function, it can be decomposed into a series of simple sine-waves, each characterized by an amplitude, a frequency, and a phase angle. Thus Fourier analysis is a very useful tool for identifying and quantifying periodically occurring patterns in a noisy signal, because the frequency 
components which contribute significantly to the composite signal have relatively large amplitudes. The analysis involves determining the amplitudes and phase angles corresponding to each frequency. (The frequencies do not need to be determined, because the mathematics requires them to be integral multiples of the frequency of the periodic waveform.)

As an alternative to the expression above, the Fourier series can be written in exponential form with complex numbers as the coefficients of each term, as shown below. In this form, the relation between the real and imaginary parts of the coefficients expresses the phase-angle information. (In this equation $i$ is the square root of -1.)

$$
\begin{aligned}
& f(t)=C_{0}+\sum_{n=1}^{\infty} C_{n} \exp \left(i 2 \pi F_{n} t\right) \\
& \theta_{n}=\tan ^{-1}\left[\operatorname{lm}\left(C_{n}\right) / \operatorname{Re}\left(C_{n}\right)\right]
\end{aligned}
$$

From the rigorous mathematical standpoint, the Fourier series exists only for periodic functions. Although the function represented by the series of DPLs from a nuclear power plant is not periodic, for the purpose of the Fourier analysis it is permissible to assume that it is, with a period at least equal to the "window" of time over which the signal is to be analyzed. As noted above, the frequencies of the Fourier series components are determined by the period of the signal, which in this case is more or less arbitrarily assumed. Thus the specific frequencies of the components are artifacts of the analytical approach, and are not necessarily significant.

The most convenient way to obtain the Fourier coefficients of a series of discrete equalinterval samples of a periodic function is the Fast Fourier Transform (FFT), a numerical method which lends itself to computer implementation. SAIC used the FFT routine of MATLAB'M, a commercial mathematical analysis software package running on an IBM-PC-AT personal computer, to process the DPL data from the various plants.

For each plant, the DPL data for each of the years 1985 through 1987 was analyzed by the FFT routine. One calendar year was selected as a first-trial time "window" for convenience in data entry; the effect of choosing other analysis intervals remains an open question. 
The final step in the analysis performed to date was to convert the series of complex Fourier coefficients to a power spectral density profile, which can be plotted as a graph of power versus frequency. In a conventional communications signal processing application, this is an expression of the signal power contained by each Fourier component. In the current analysis, the power spectral density simply highlights the frequency components which are the most important contributors to the total DPL waveform.

\subsubsection{Objectives of the Fourier Analysis}

As noted above, Fourier analysis is a good technique for identifying periodic elements of a signal which are masked by random "noise." SAIC hypothesized that the DPL data from nuclear power plants contains the two types of periodicities discussed below, both of which should be found and addressed.

(1) Periodic DPL fluctuations caused by regularly-occurring external phenomena such as seasonal power demand variations are spurious signals from the maintenance performance standpoint, and should be identified as such so they can be filtered out of the indicator signal.

(2) Periodic fluctuations which are net attributable to external phenomena presumably represent a regular pattern of major equipment outages or deratings, suggestive of a continuing failure of plant maintenance (as broadly defined) to diagnose and correct them. These periodic elements should be identified and highlighted.

\subsection{Results and Preliminary Interpretation}

\subsubsection{Power Spectral Density (PSD) Profiles}

The PSD plots for Plant S for calendar years 1985, 1986 and 1987 are shown in Figures 4,5 , and 6 respectively, and the plots for the other seven units and the same years are reproduced in Appendix 1. The plots show several salient features which may be related to maintenance performance, and appear to justify further investigation.

(1) Extended plant outages during the analysis interval -- planned or forced -- appear to correlate with most of the "power" being concentrated in one or a very few low-frequency Fourier components on the PSD plot. 


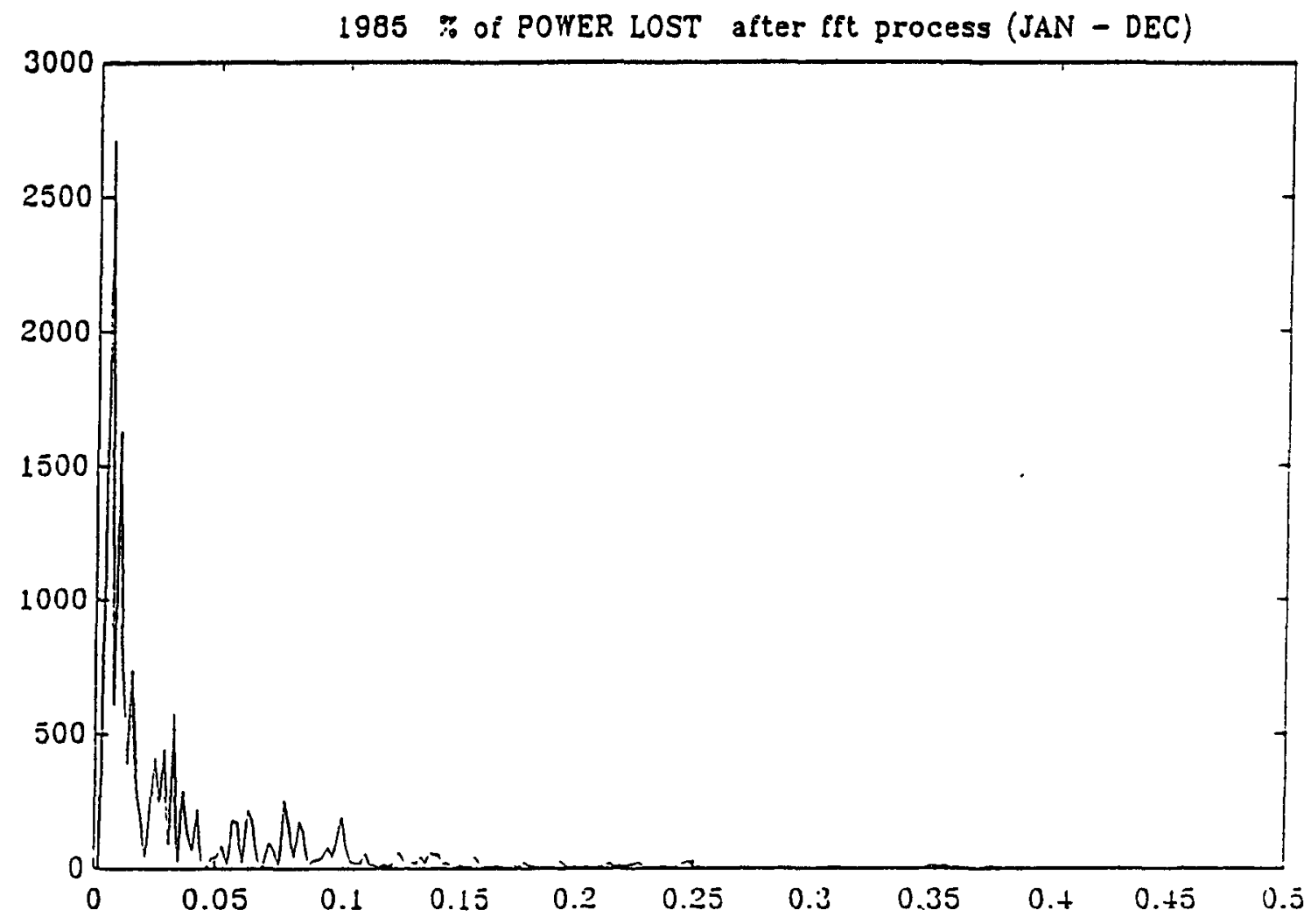

Figure 4 


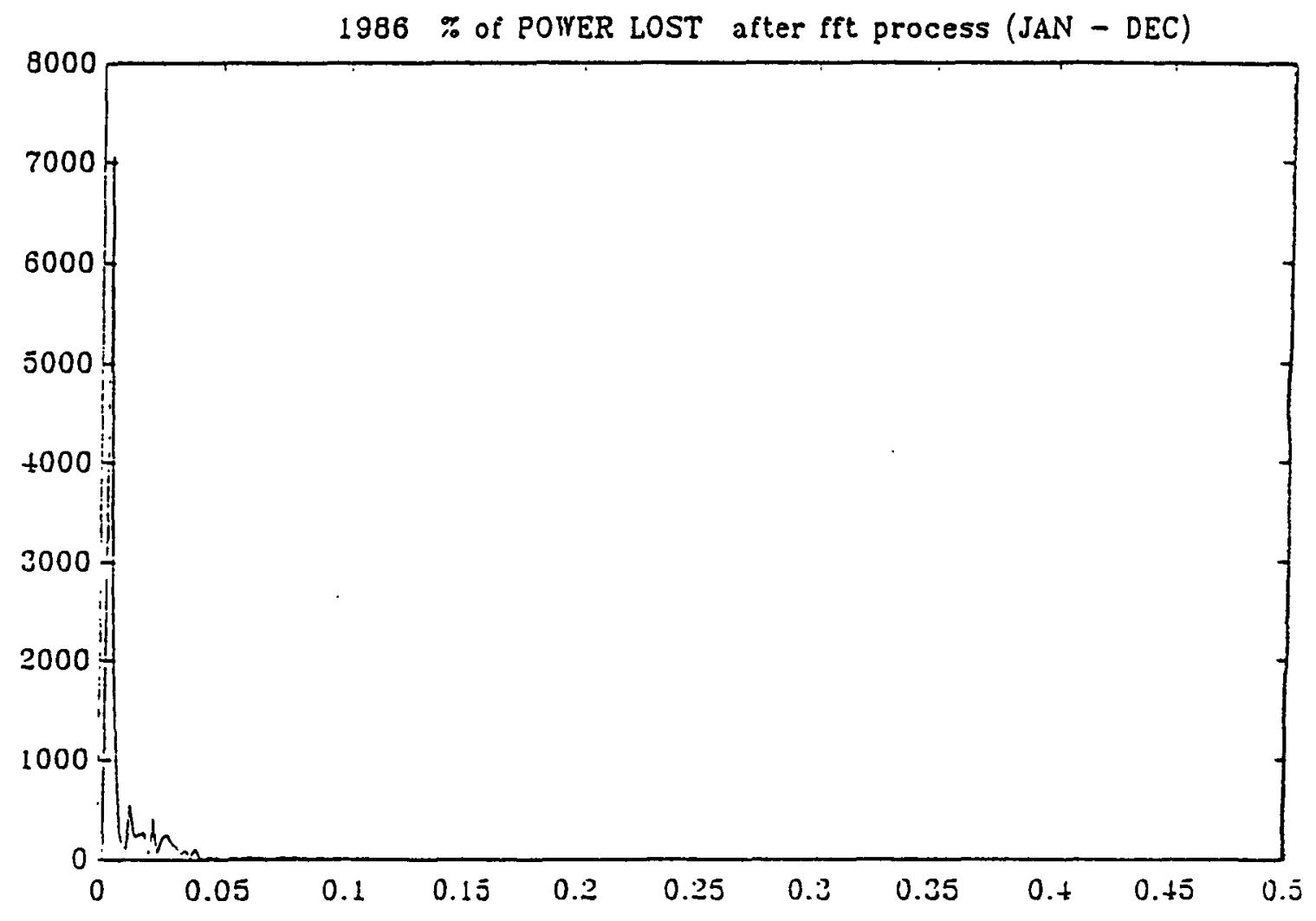

Figure 5

Att-1-22 


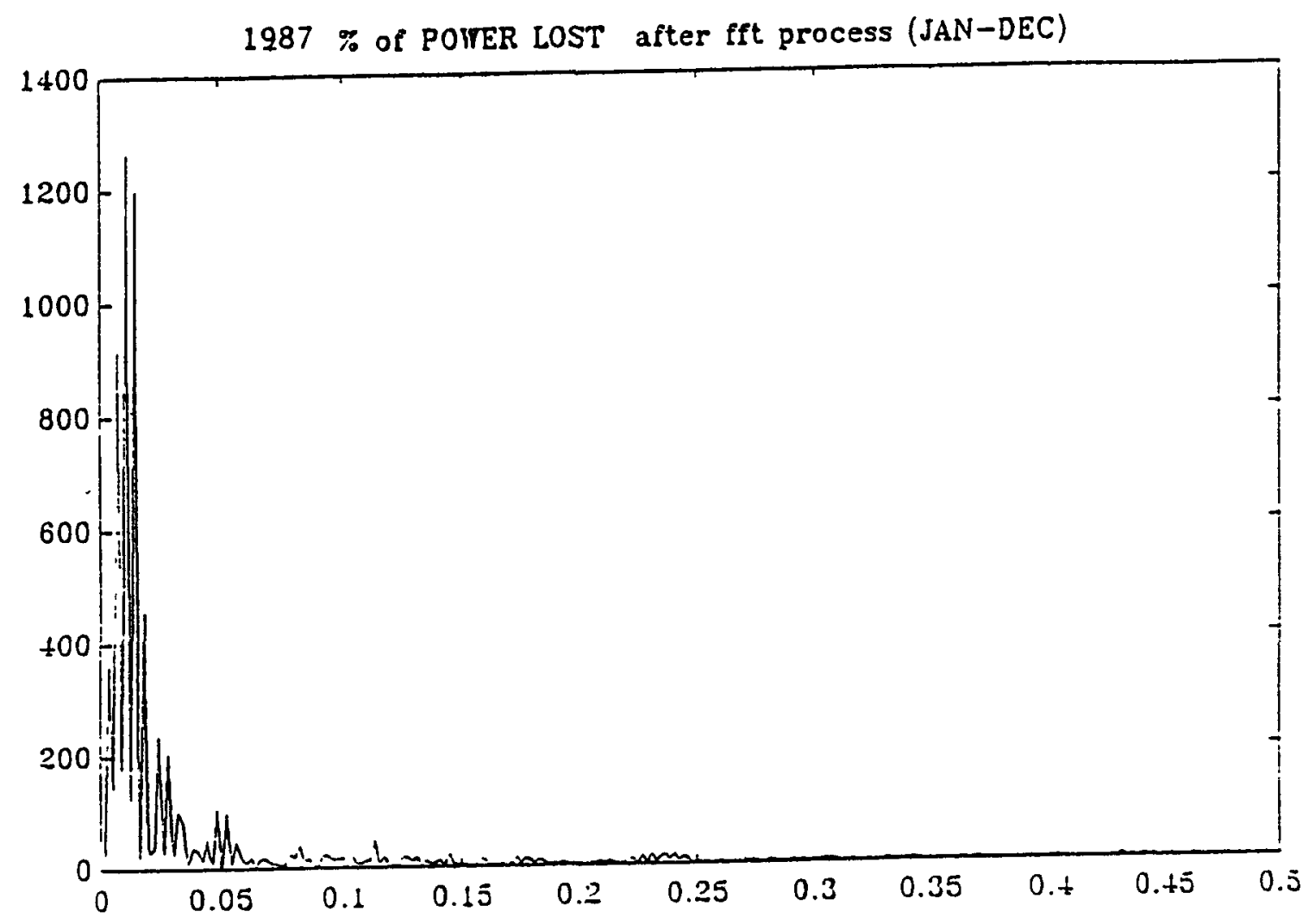

Figure 6 
(2) Many short-duration fluctuations in DPL, denoting many outage events, appear to correlate with a broader, but still concentrated distribution of "power" among Fourier components in the PSD plot.

(3) A "good" operating year, without extended outages or numerous incidents leading to power level changes, appears correlated with a relatively flat "power" distribution. 
DATA FOR COSINE PLOTS OF YEARLY FREQUENCIES

\begin{tabular}{|c|c|c|c|c|c|c|c|}
\hline PLANT & YEAR & $\begin{array}{c}\text { FIRST } \\
\text { AMPLITUDE }\end{array}$ & $\begin{array}{c}\text { SLCOND } \\
\text { AMPLITUDE }\end{array}$ & $\begin{array}{c}\text { THIRI) } \\
\text { AMPLITUDE }\end{array}$ & $\begin{array}{c}\text { FIRST } \\
\text { FREQUENCY }\end{array}$ & $\begin{array}{c}\text { SECOND } \\
\text { FREQUENCY }\end{array}$ & $\begin{array}{c}\text { THIRD } \\
\text { FREQUENCY }\end{array}$ \\
\hline $\mathbf{S}$ & 1985 & $2710 E+4$ & $.1629 E+4$ & $.7357 E+3$ & 5859 E-2 & .9766 E-2 & $.1563 \mathrm{E}-1$ \\
\hline & 1986 & $.7060 E+4$ & $.8390 E+3$ & $.5513 E+3$ & .3906 E-2 & .7813 E-2 & $.1367 \mathrm{E}-1$ \\
\hline & 1987 & $1268 E+4$ & $.1196 E+4$ & $.9148 E+3$ & .1172 E-1 & $.1563 E-1$ & $.7813 \mathrm{E}-2$ \\
\hline & 1985 & $.4124 E+4$ & $.9345 E+3$ & $.4503 E+3$ & .3906 E-2 & .1172 E-1 & .1758 E-1 \\
\hline & 1986 & $.4780 E+3$ & $.4128 E+3$ & $4691 E+3$ & 9766 E-2 & .1367 E-1 & $.3711 \mathrm{E}-1$ \\
\hline & 1987 & $.9530 E+4$ & $.5378 E+3$ & $.9543 E+2$ & $3906 \mathrm{E}-2$ & $.1367 \mathrm{E}-1$ & $.1758 \mathrm{E}-1$ \\
\hline U & 1985 & $5181 E+4$ & $2144 E+4$ & $2940 E+3$ & $3906 \mathrm{E}-2$ & $.1367 \mathrm{E}-1$ & $.2743 \mathrm{E}-1$ \\
\hline & $1986^{1}$ & $.1322 E+1$ & $.1322 E+1$ & $.1321 E+1$ & .1953 E-2 & $.3906 \mathrm{E}-2$ & $.5859 \mathrm{E}-2$ \\
\hline & 1987 & $.1683 E+4$ & $.7065 E+3$ & $.4571 E+3$ & $9766 \mathrm{E}-2$ & $.3906 \mathrm{E}-2$ & .1758 E-1 \\
\hline V & 1985 & $.7081 E+4$ & $.1221 E+4$ & $.3832 E+3$ & 5859 E-2 & $.1172 E-1$ & $.2539 E-2$ \\
\hline & 1986 & $6969 E+4$ & $.1616 E+4$ & $.4817 E+3$ & 5859 E-2 & $.1367 \mathrm{E}-1$ & $.1758 \mathrm{E}-1$ \\
\hline & 1987 & $9686 E+4$ & $.5013 E+3$ & $.5102 E+3$ & 5859 E-2 & $.1367 \mathrm{E}-1$ & $.2148 \mathrm{E}-1$ \\
\hline W & 1985 & $2329 E+4$ & $.2294 E+4$ & $.1212 E+4$ & $3906 \mathrm{E}-2$ & $.9766 \mathrm{E}-2$ & $.2148 \mathrm{E}-1$ \\
\hline & 1986 & $.7704 E+4$ & $.2169 E+3$ & $.1392 E+3$ & .3906 E-2 & $.1563 \mathrm{E}-1$ & .2930 E-1 \\
\hline & 1987 & $4078 E+4$ & $.5053 E+3$ & $.1683 E+3$ & 5859 E-2 & $.2539 E-1$ & .1758 E-1 \\
\hline
\end{tabular}


DATA FOR COSINE PI.OTS OF YEARI.Y FREQUENCIES

\begin{tabular}{|c|c|c|c|c|c|c|c|}
\hline PLANT & YEAR & $\begin{array}{c}\text { FIRST } \\
\text { AMPLITUDE }\end{array}$ & $\begin{array}{c}\text { SECOND } \\
\text { AMPLITUDE }\end{array}$ & $\begin{array}{c}\text { THIRD } \\
\text { AMPLITUDE }\end{array}$ & $\begin{array}{c}\text { FIRST } \\
\text { FREQUENCY }\end{array}$ & $\begin{array}{c}\text { SECOND } \\
\text { FREQUENCY }\end{array}$ & $\begin{array}{c}\text { THIRD } \\
\text { FREQUENCY }\end{array}$ \\
\hline \multirow[t]{3}{*}{$x$} & 1985 & $.1905 E+3$ & $.1721 E+3$ & $.1304 E+3$ & $.7813 \mathrm{E}-2$ & $.1367 \mathrm{E}-1$ & $.2539 \mathrm{E}-1$ \\
\hline & 1986 & $.5118 E+4$ & $.3257 E+4$ & $.1068 E+4$ & $.7813 E-2$ & .3906 E-2 & .1563 E-2 \\
\hline & $1987^{(2)}$ & $.2955 E+4$ & $.1387 E+4$ & $.4734 E+3$ & $.7813 \mathrm{E}-2$ & .1172 E-1 & $.1758 \mathrm{E}-1$ \\
\hline \multirow[t]{3}{*}{$Y$} & 1985 & $.1951 E+4$ & $.1912 E+4$ & $.1094 E+4$ & .3906 E-2 & .9766 E-2 & $.1758 \mathrm{E}-1$ \\
\hline & 1986 & $.1739 E+4$ & $.2136 E+3$ & $.1040 E+2$ & $.5859 \mathrm{E}-2$ & $.3125 \mathrm{E}-1$ & $.4492 \mathrm{E}-1$ \\
\hline & 1987 & $.3478 E+4$ & $.1176 E+4$ & $.8373 E+3$ & $.5859 \mathrm{E}-2$ & .9766 E-2 & $.1953 E-1$ \\
\hline \multirow[t]{3}{*}{$\mathbf{Z}$} & 1985 & $.4319 E+4$ & $.1278 E+4$ & $.3548 E+3$ & .5859 E-2 & $.9766 \mathrm{E}-2$ & $.2148 \mathrm{E}-1$ \\
\hline & 1986 & $.4025 E+4$ & $.3463 E+3$ & $.3182 E+3$ & .5859 E-2 & $.2539 E-1$ & $.4102 \mathrm{E}-1$ \\
\hline & 1987 & $.8957 E+4$ & $.1973 E+4$ & $.4691 E+3$ & .5859 E-2 & .9766 E-2 & $.1758 \mathrm{E}-1$ \\
\hline \multicolumn{8}{|c|}{1985} \\
\hline \multicolumn{8}{|c|}{1986} \\
\hline & 1987 & & & & & & \\
\hline \multicolumn{8}{|c|}{ NOTES: (1) Operated for only 5 days in the calendar year } \\
\hline
\end{tabular}




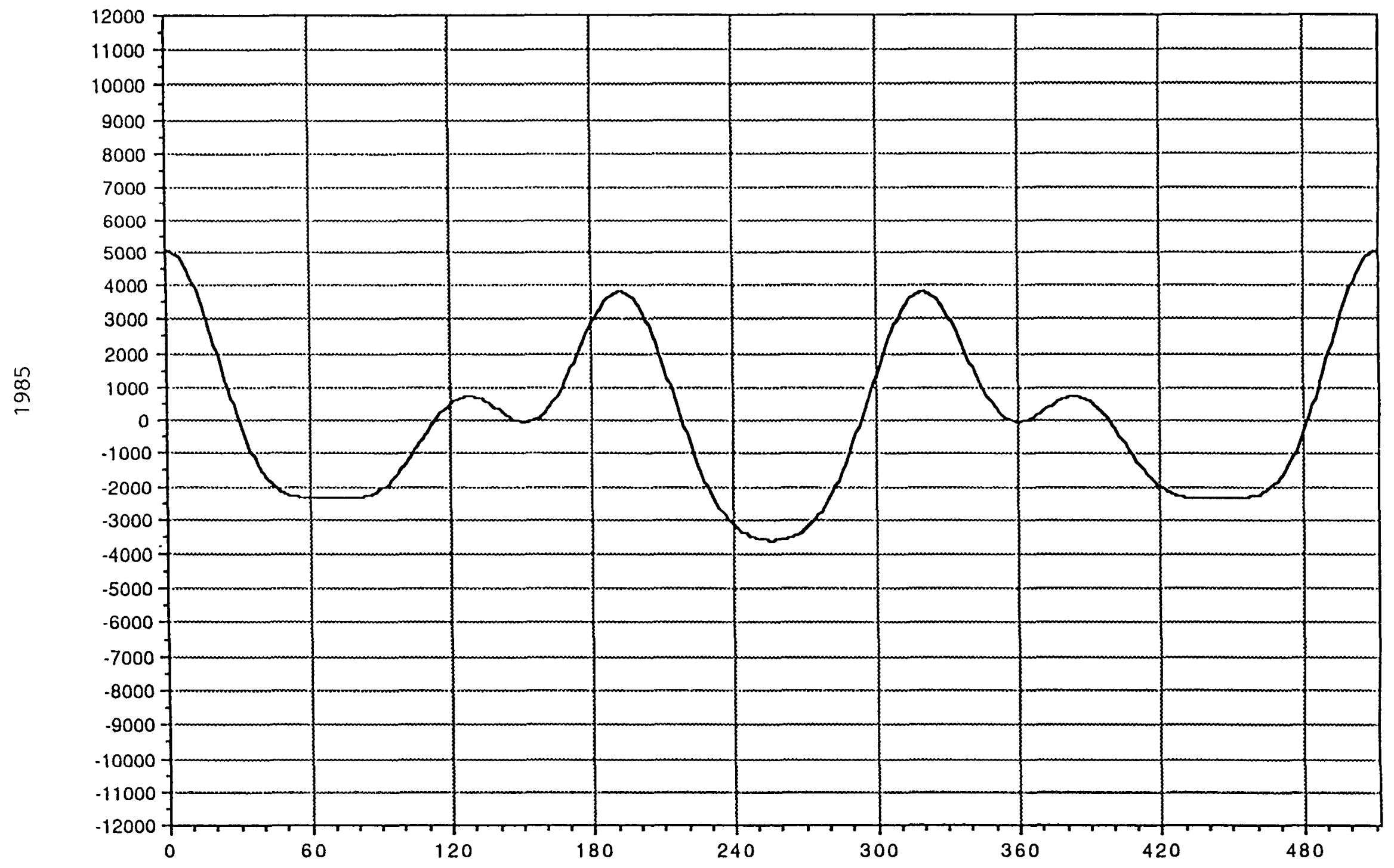




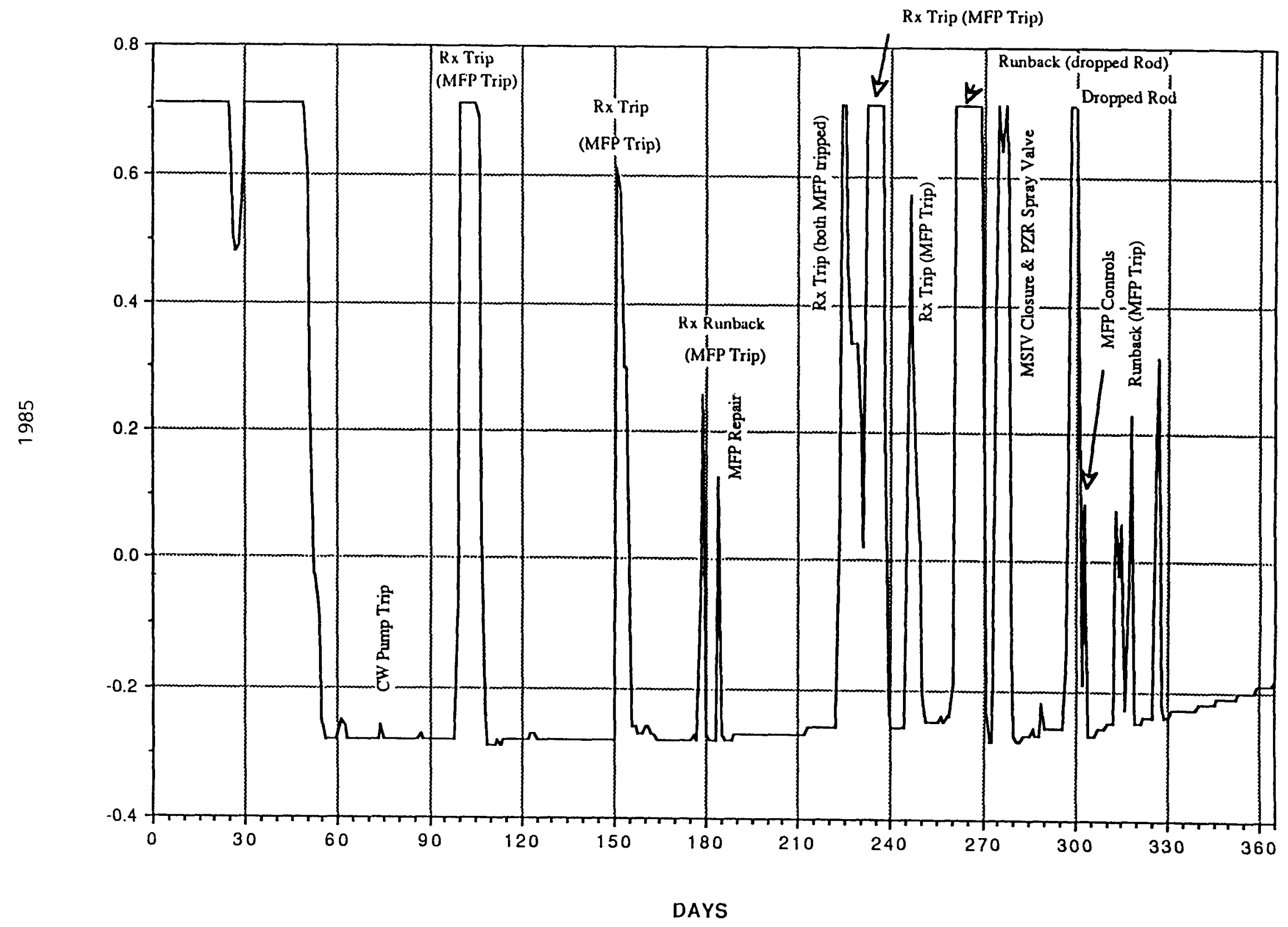

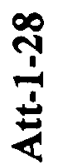




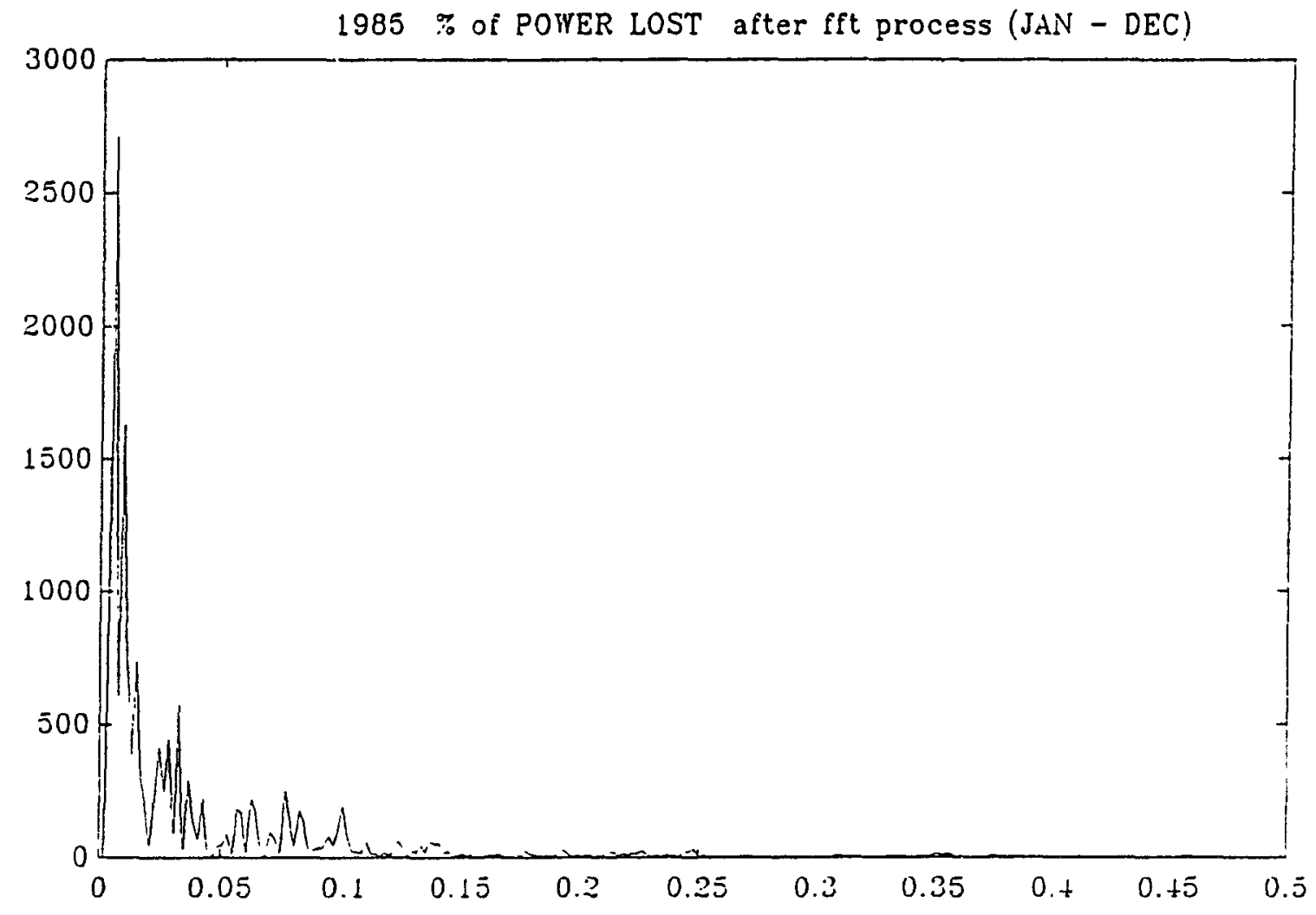

Att-1-29 


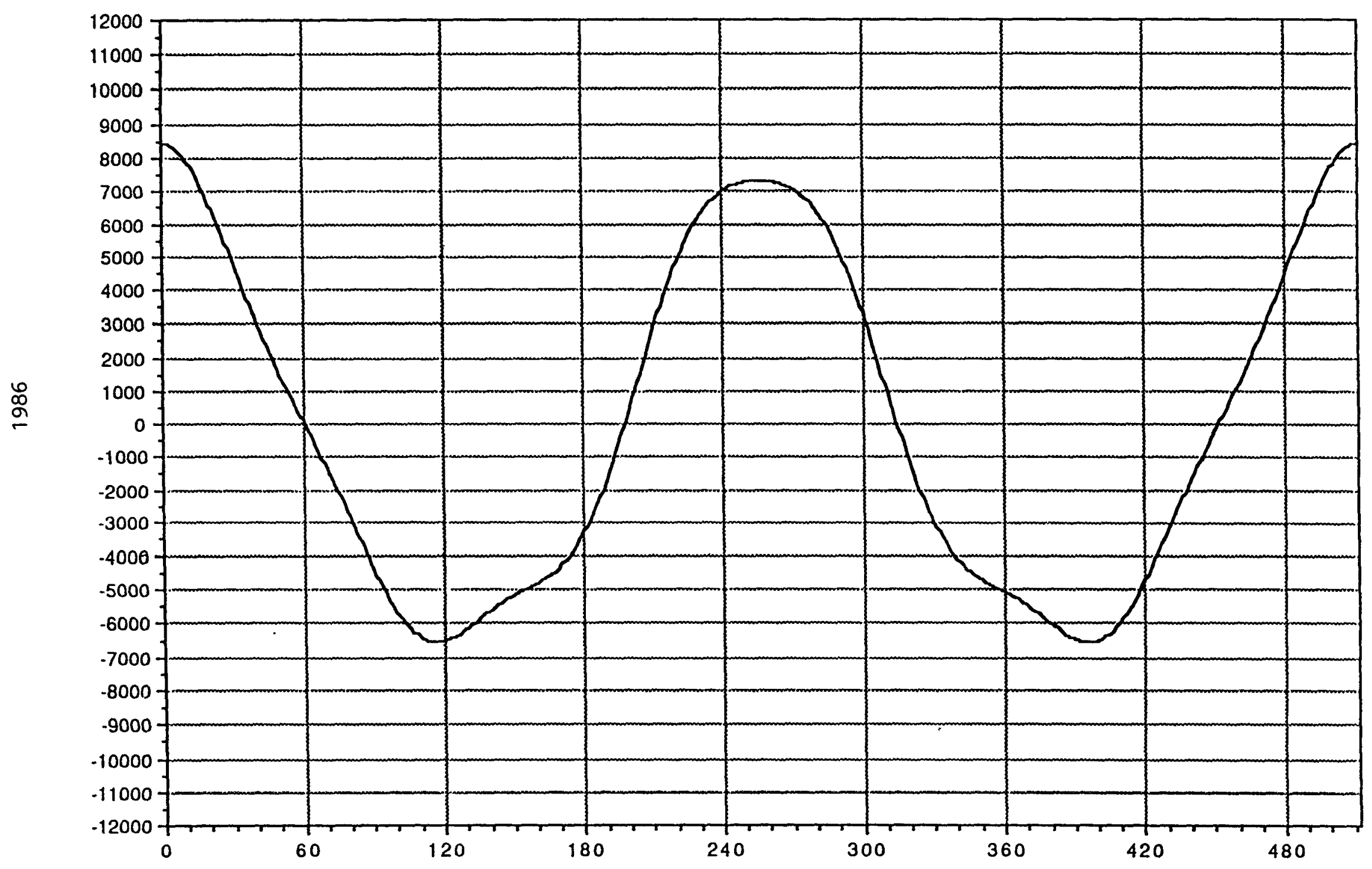




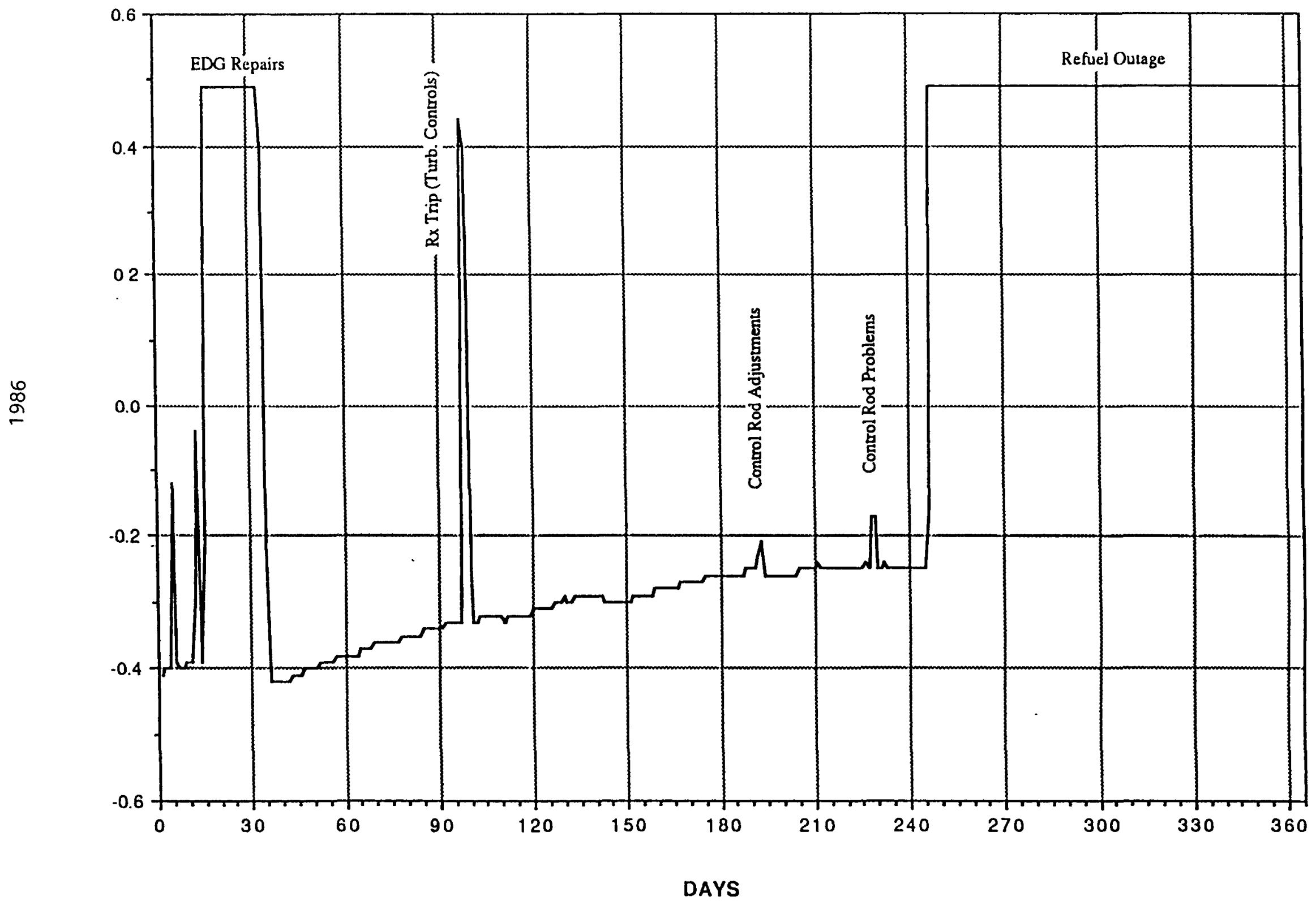

草 


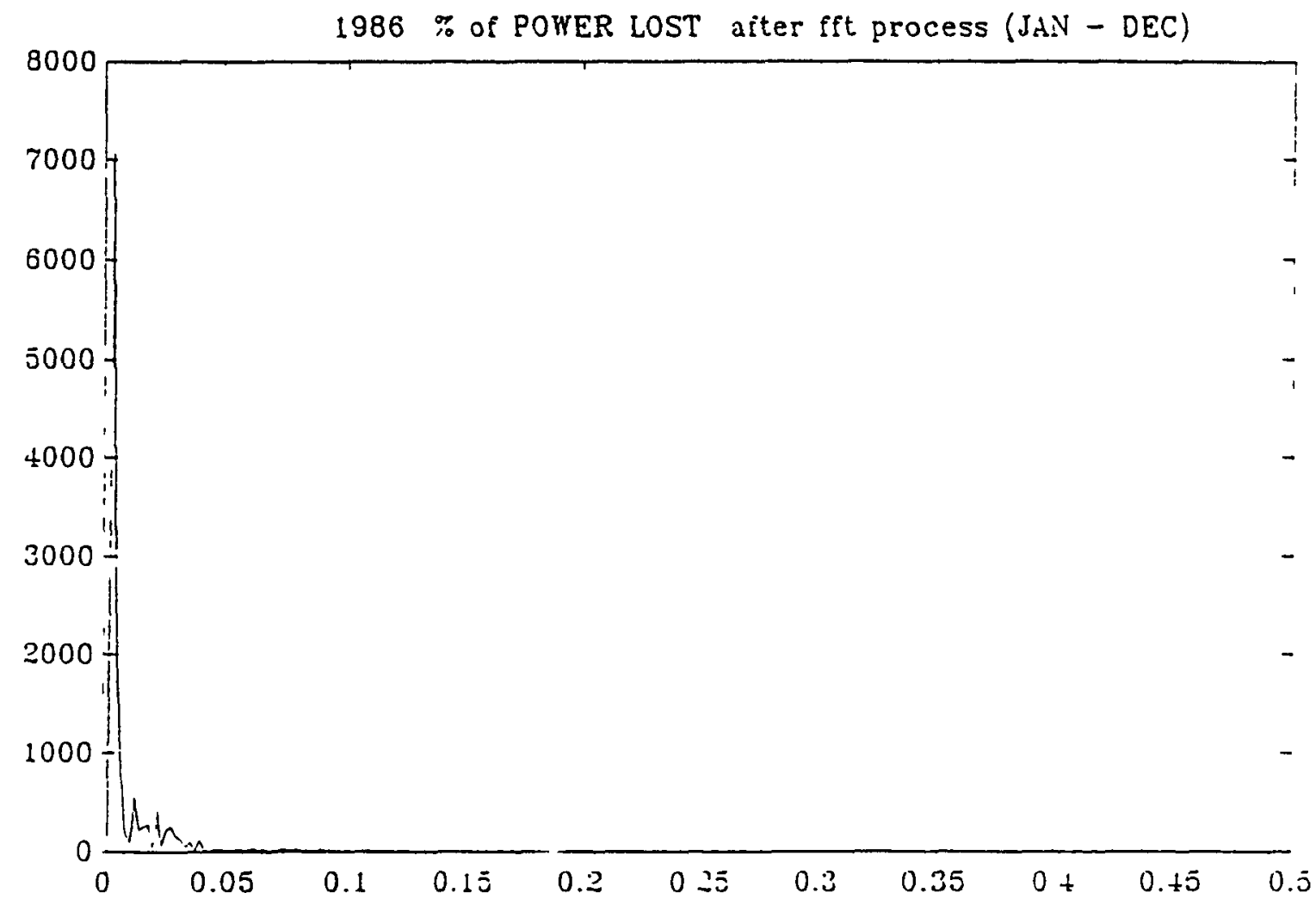




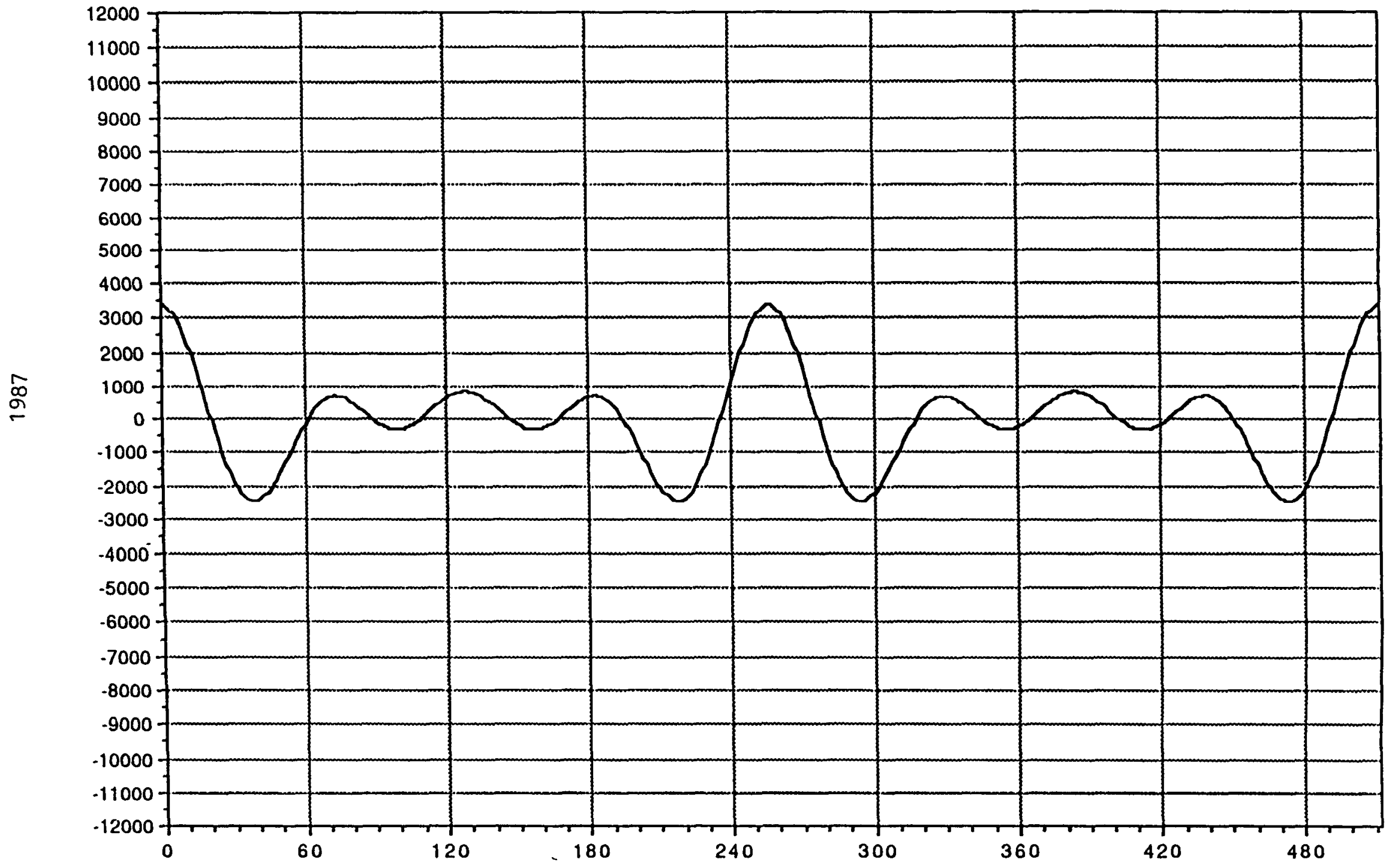




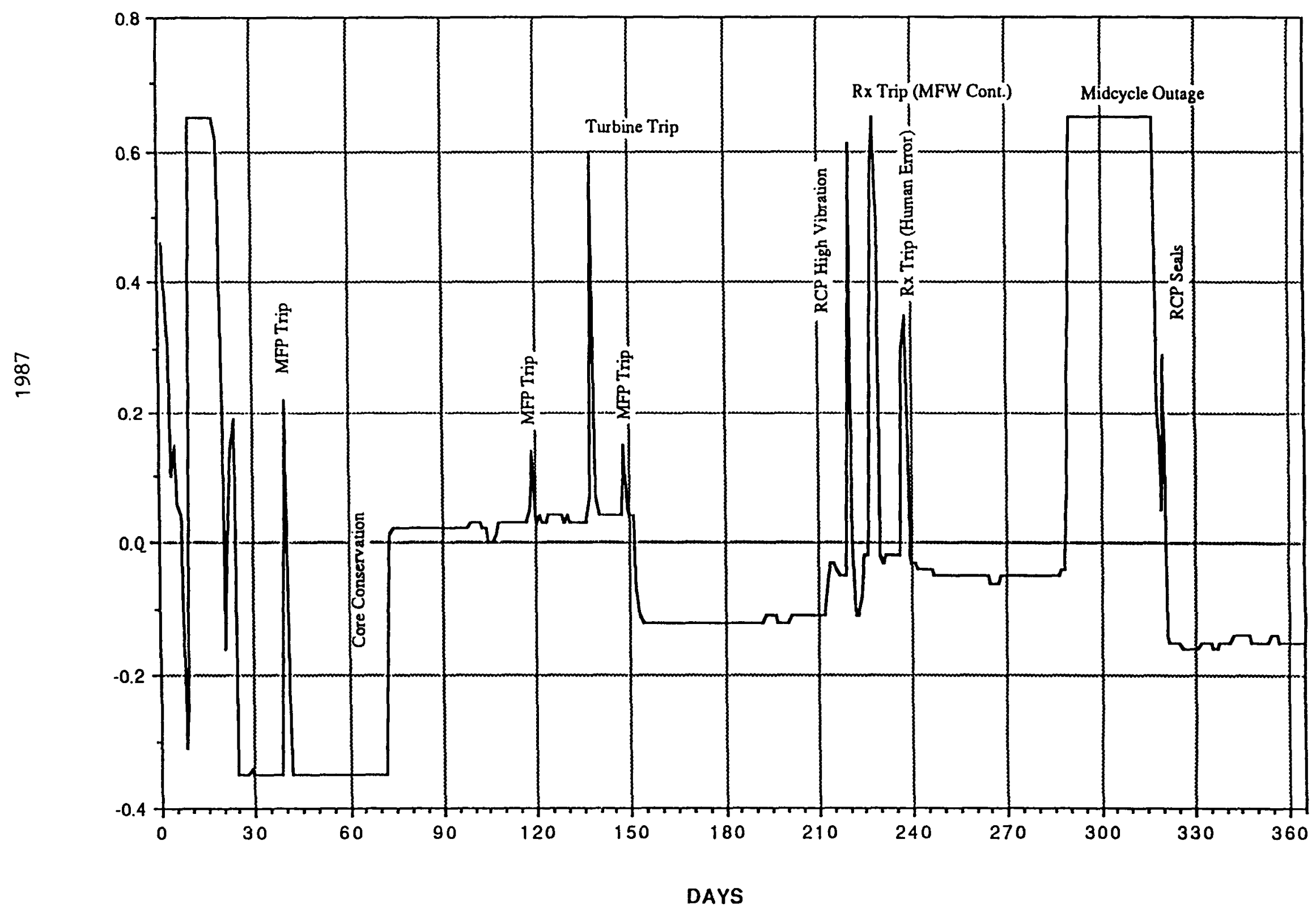

芦 


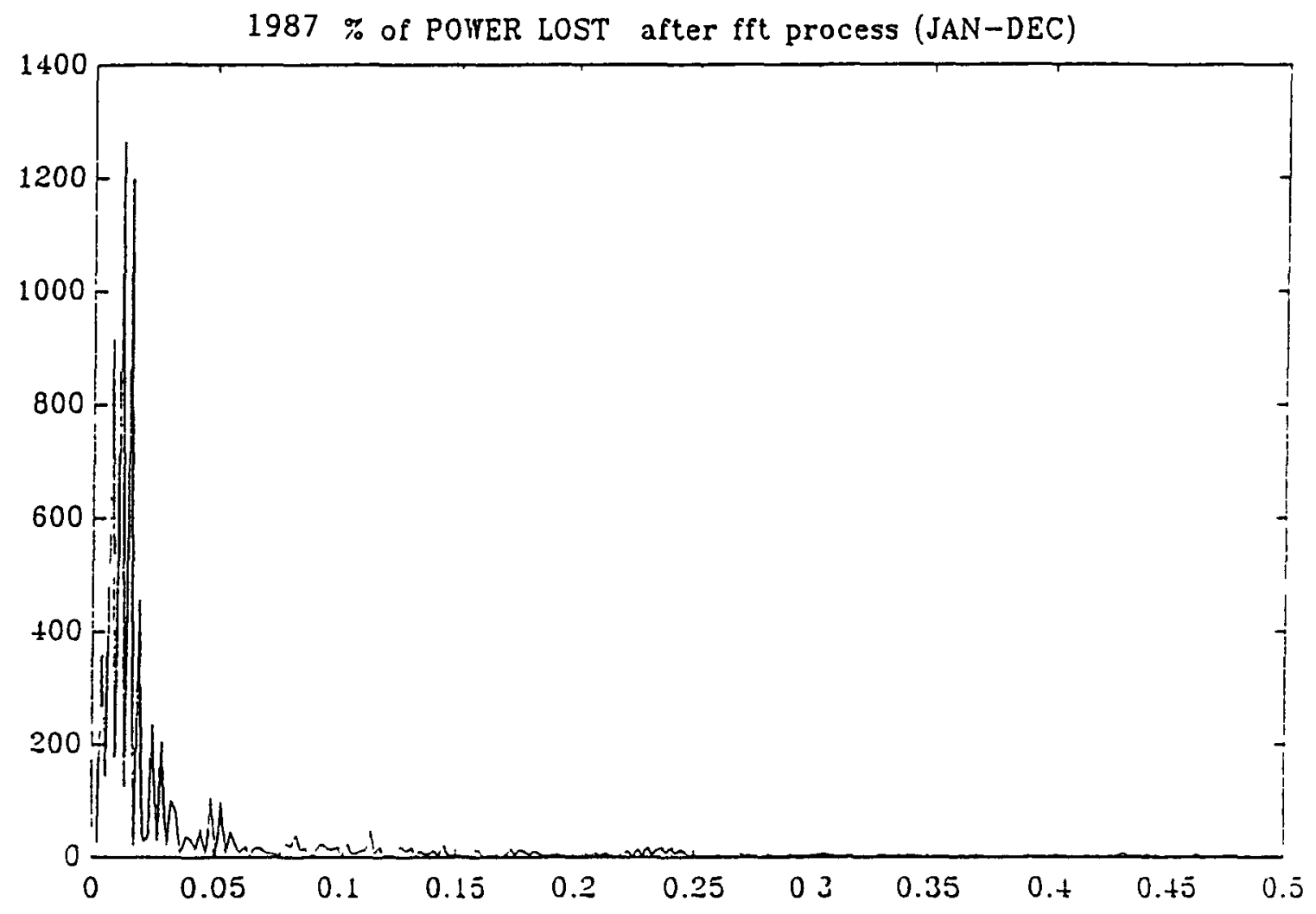

Att-1-35 
Detailed Results for Plant $\mathbf{T}$

Att-1-36 


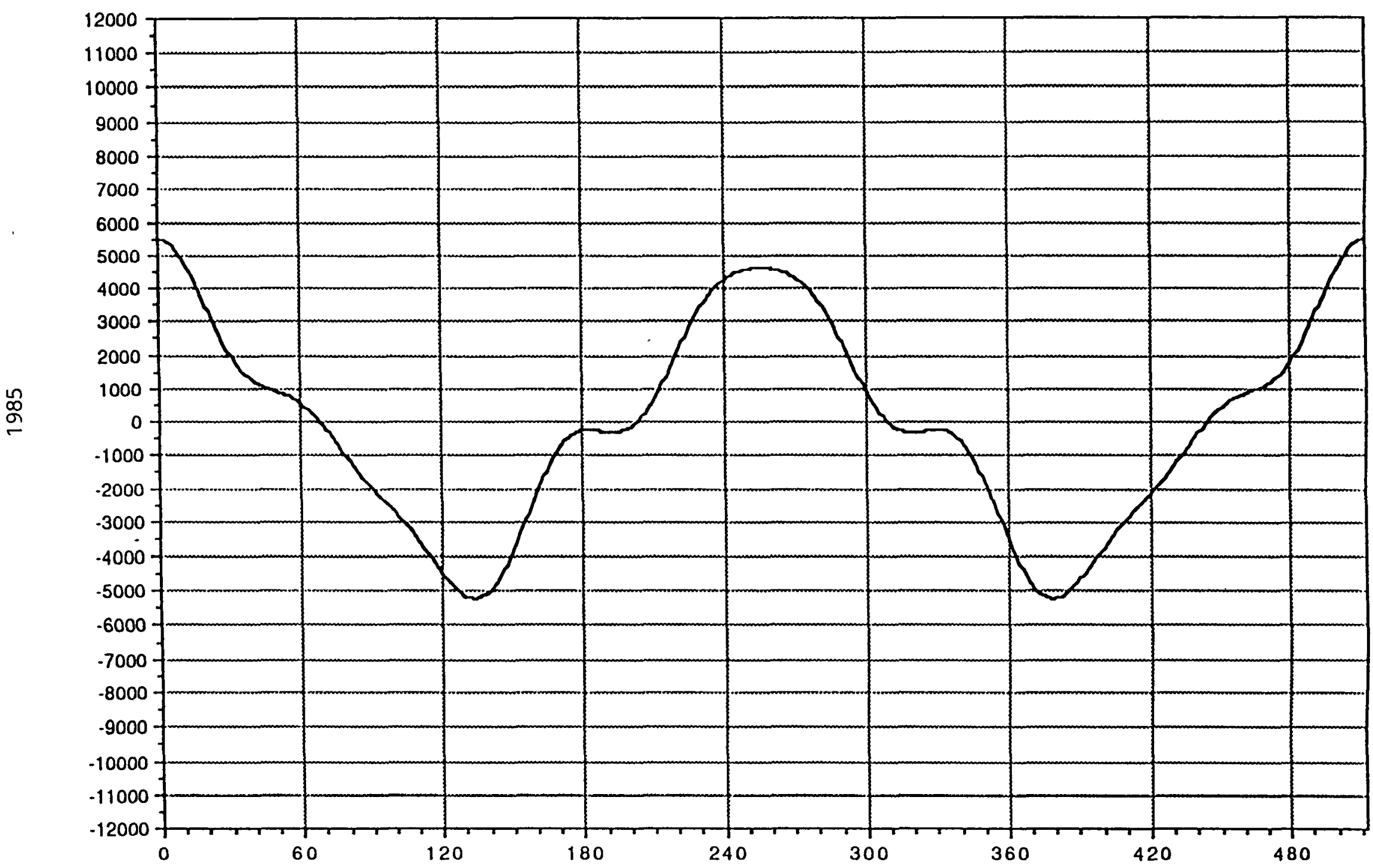




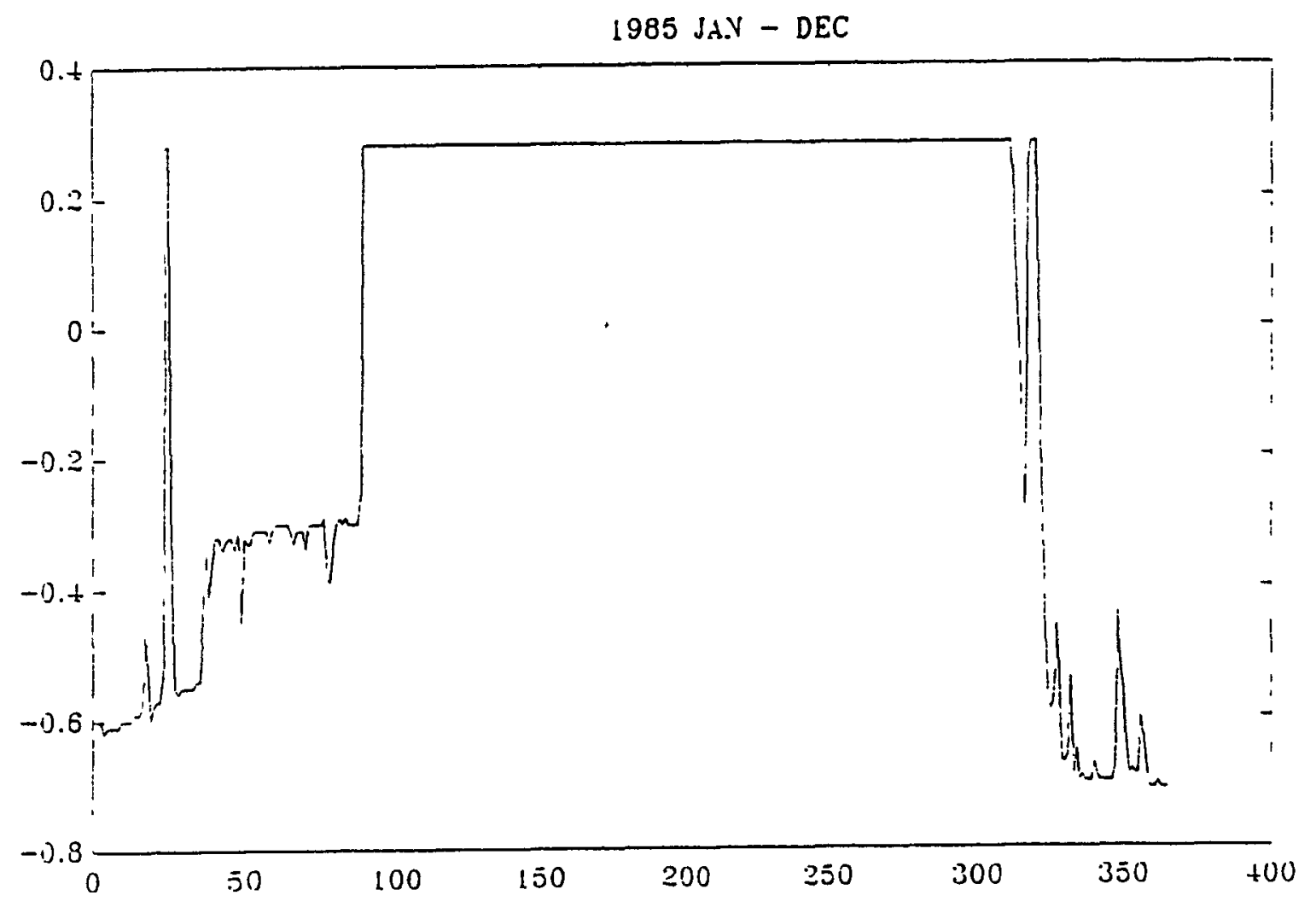

Att-1-38 
$1985 \%$ of POWER LOST after fft process (JAN - DEC)

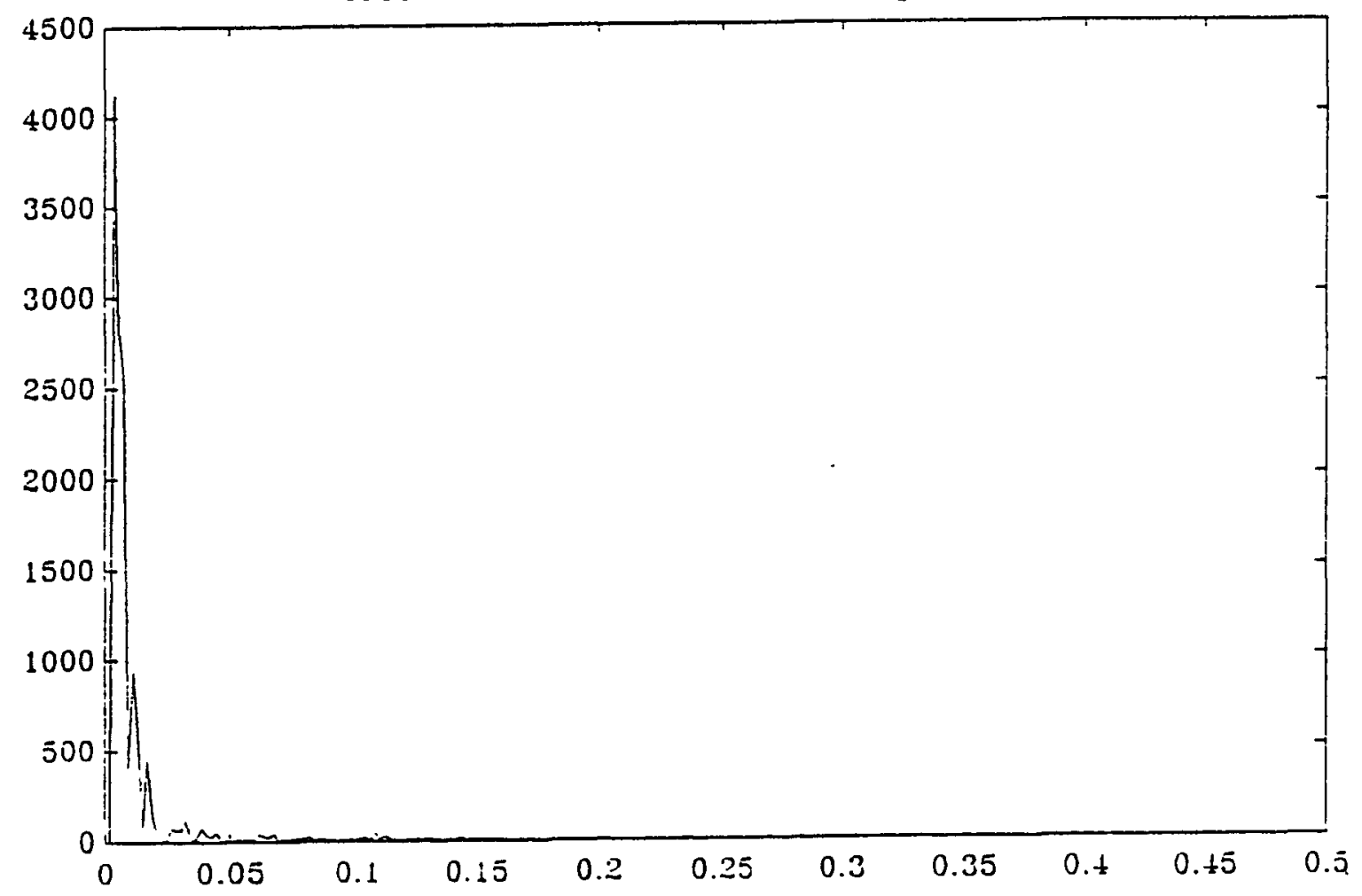




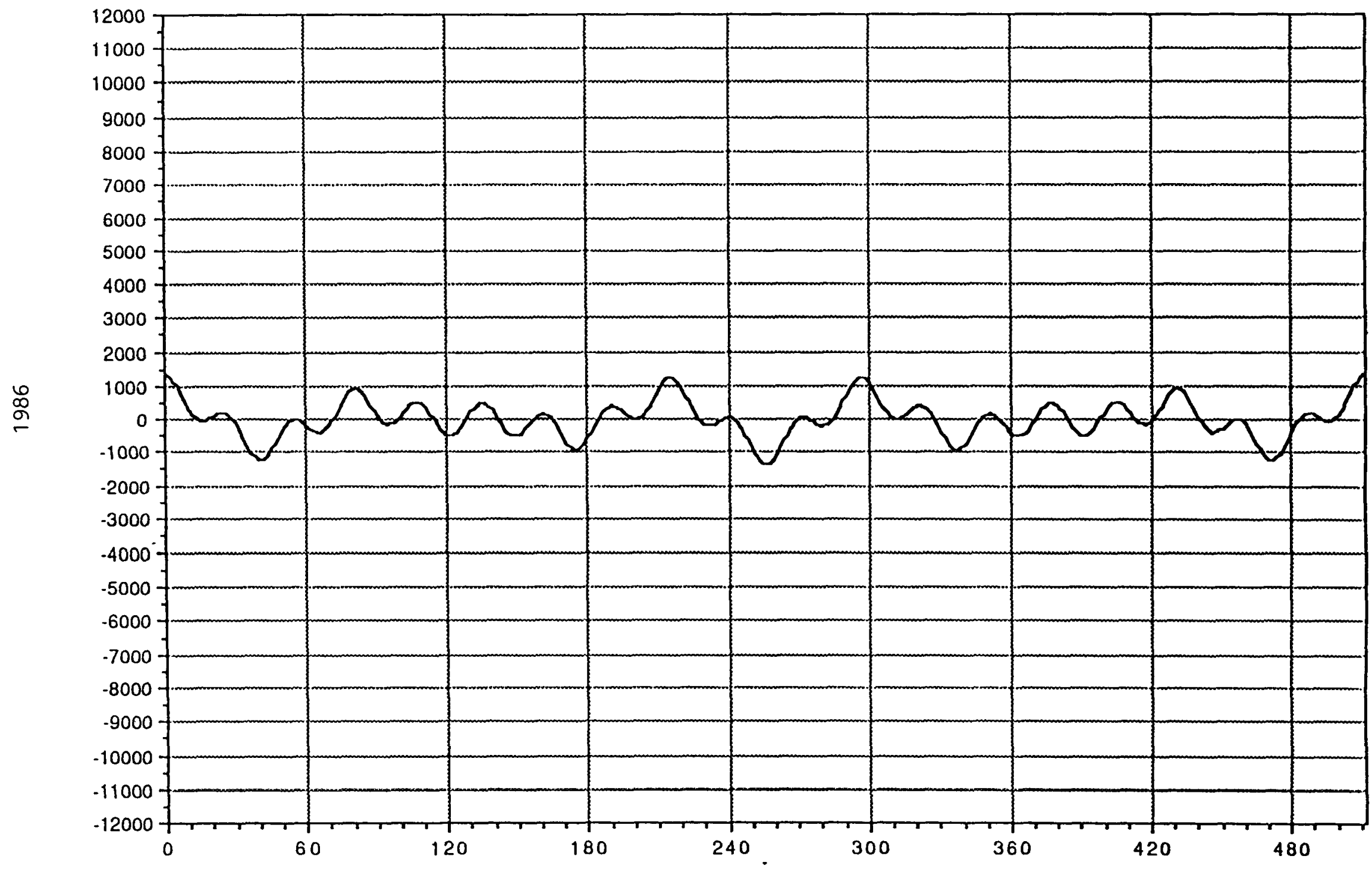




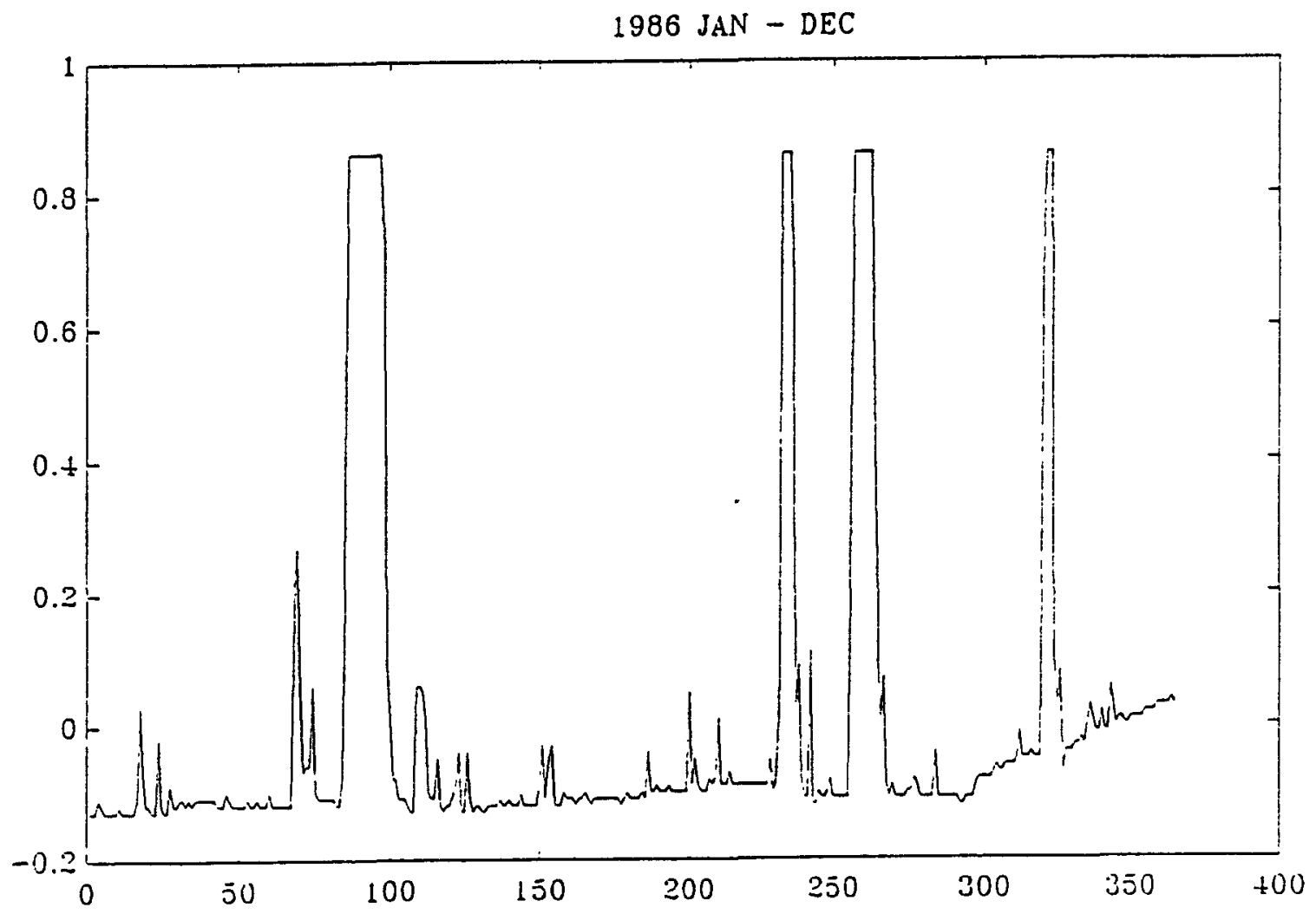









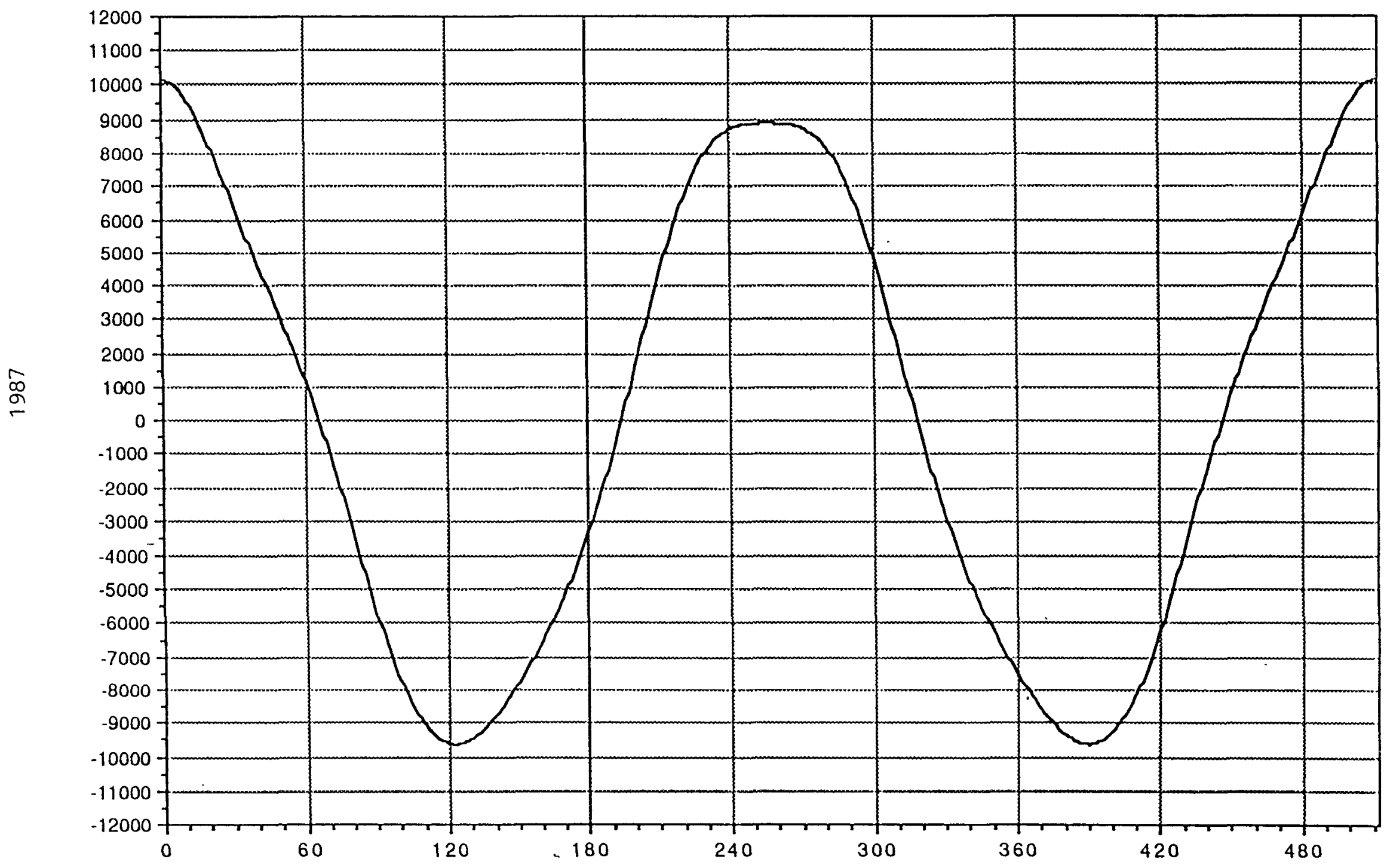




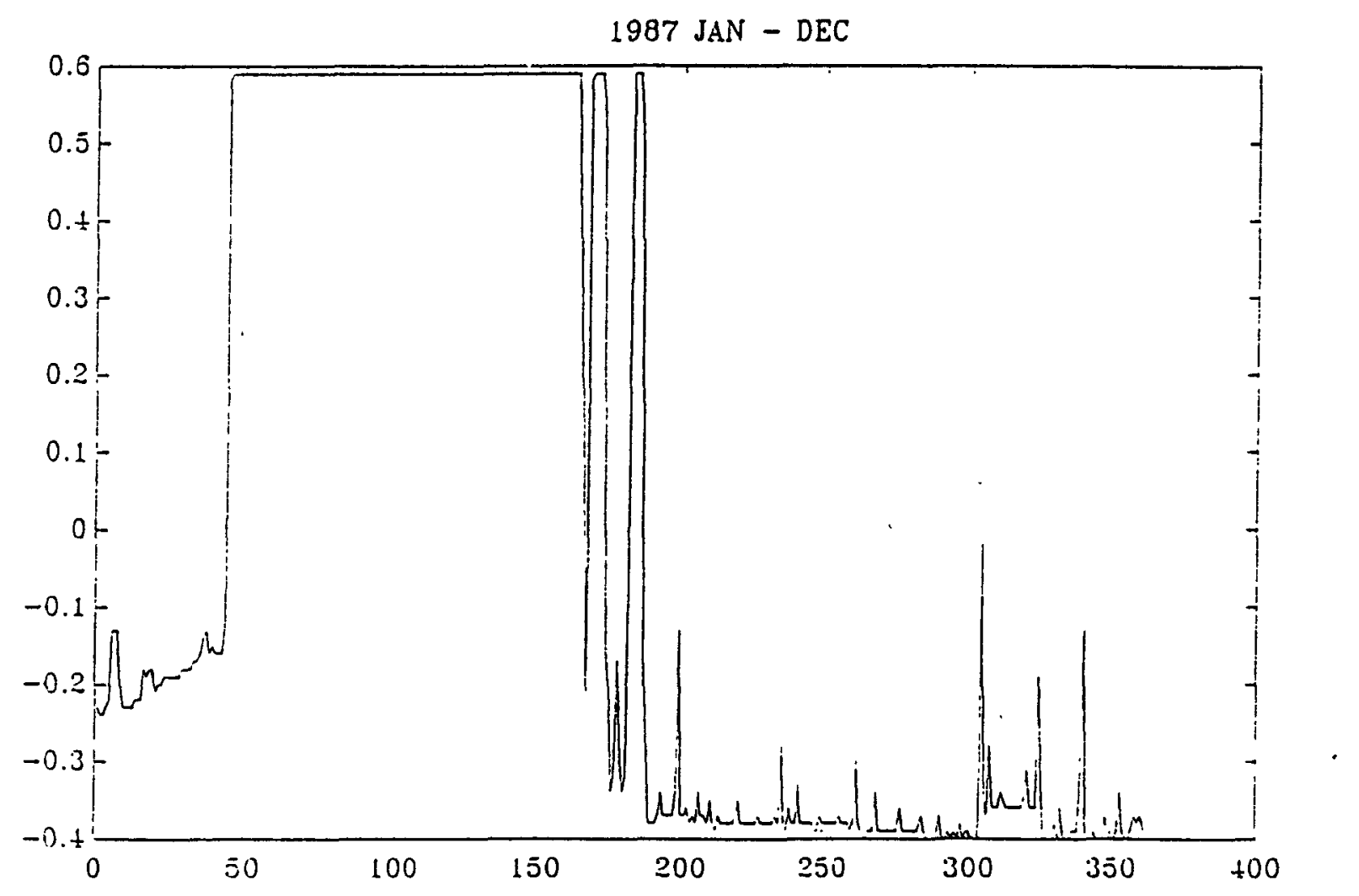




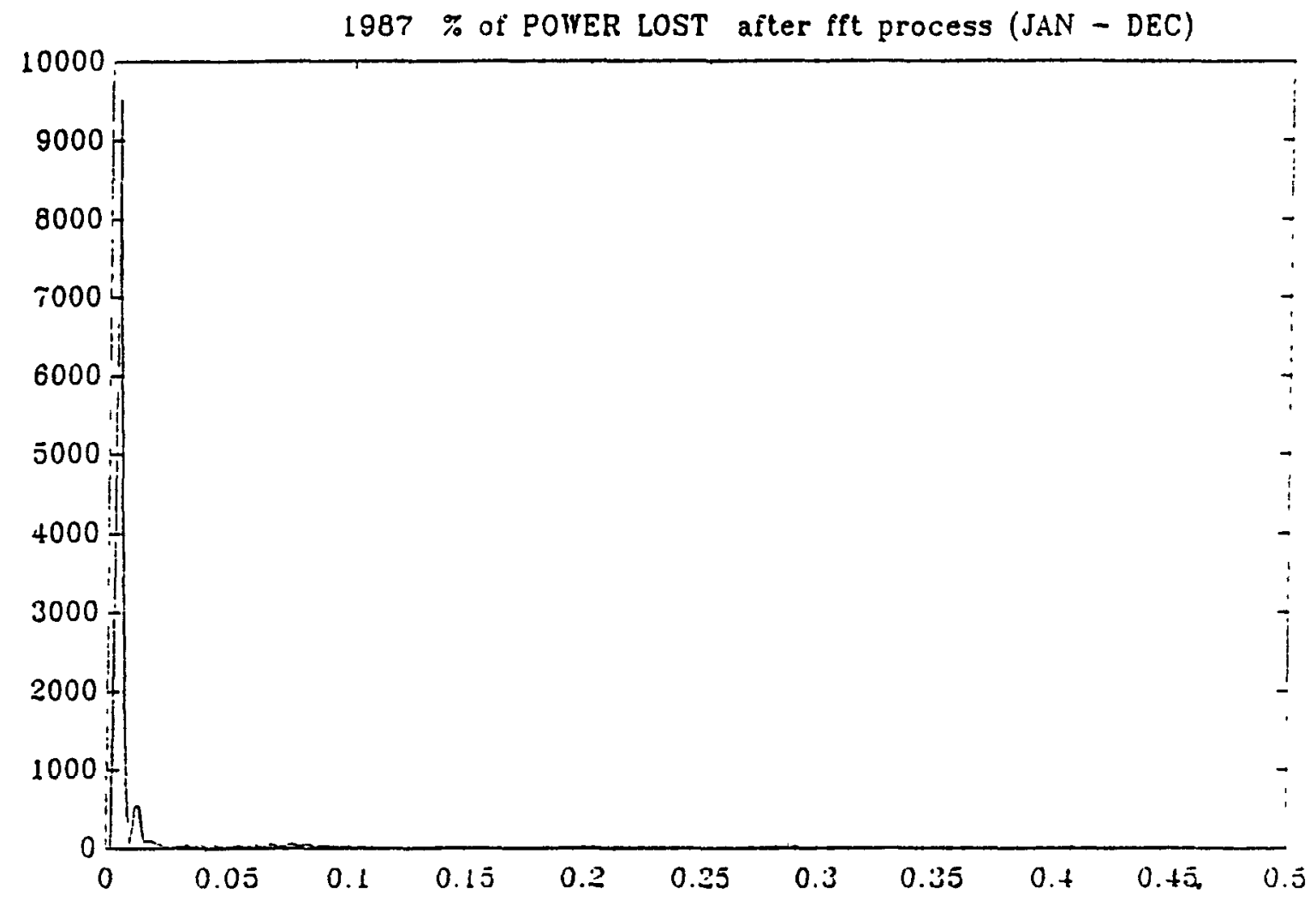


Detailed Results for Plant U 


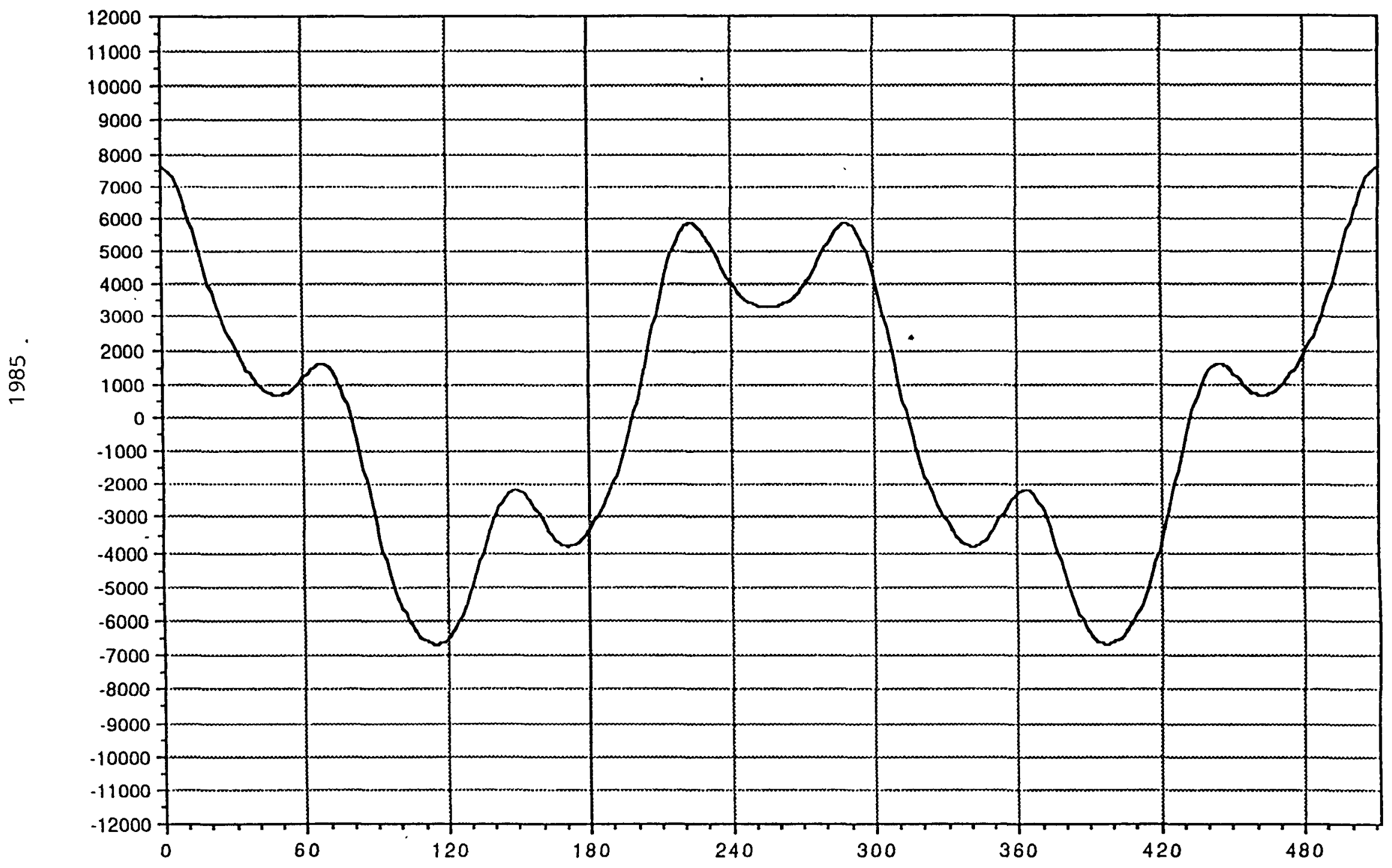




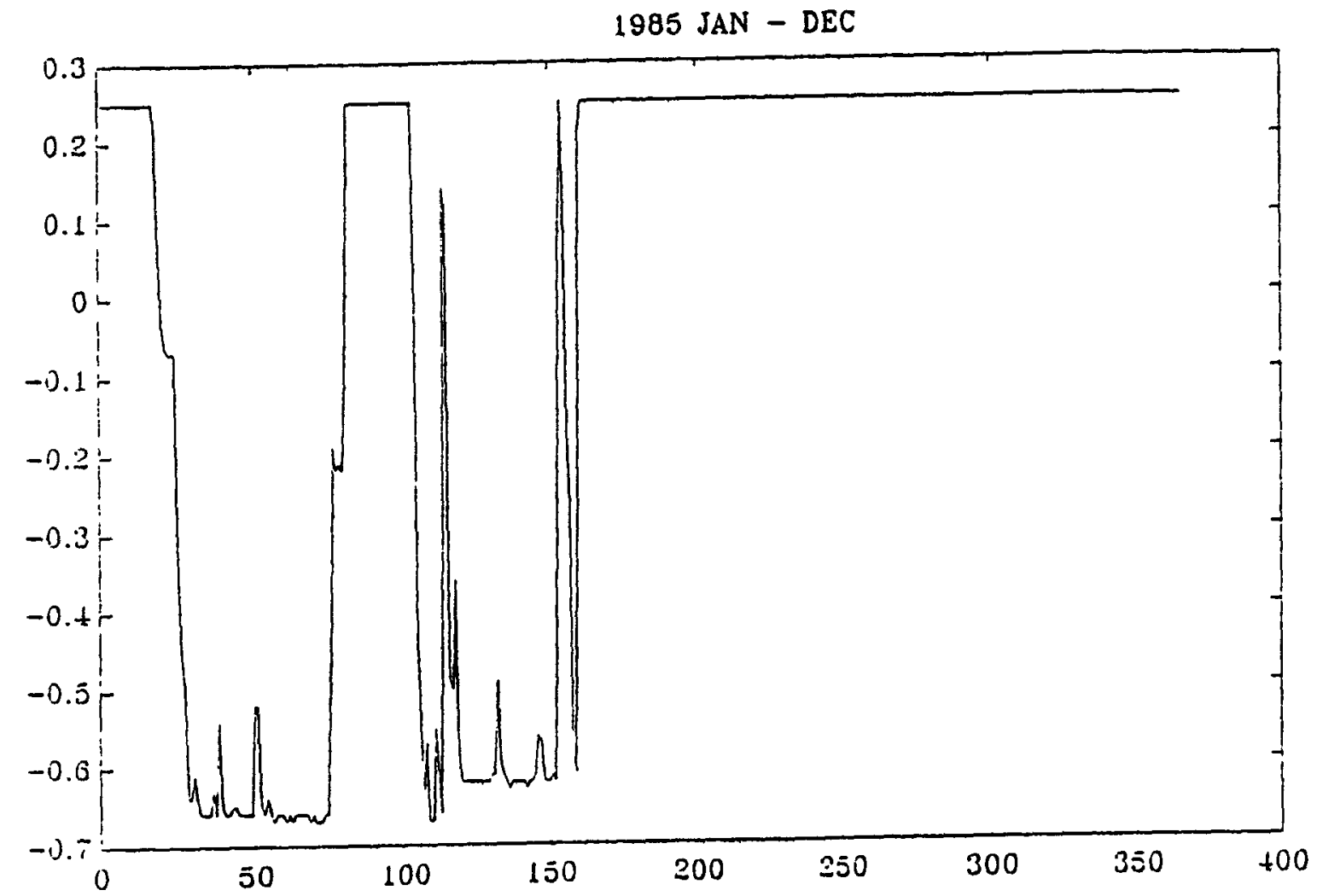




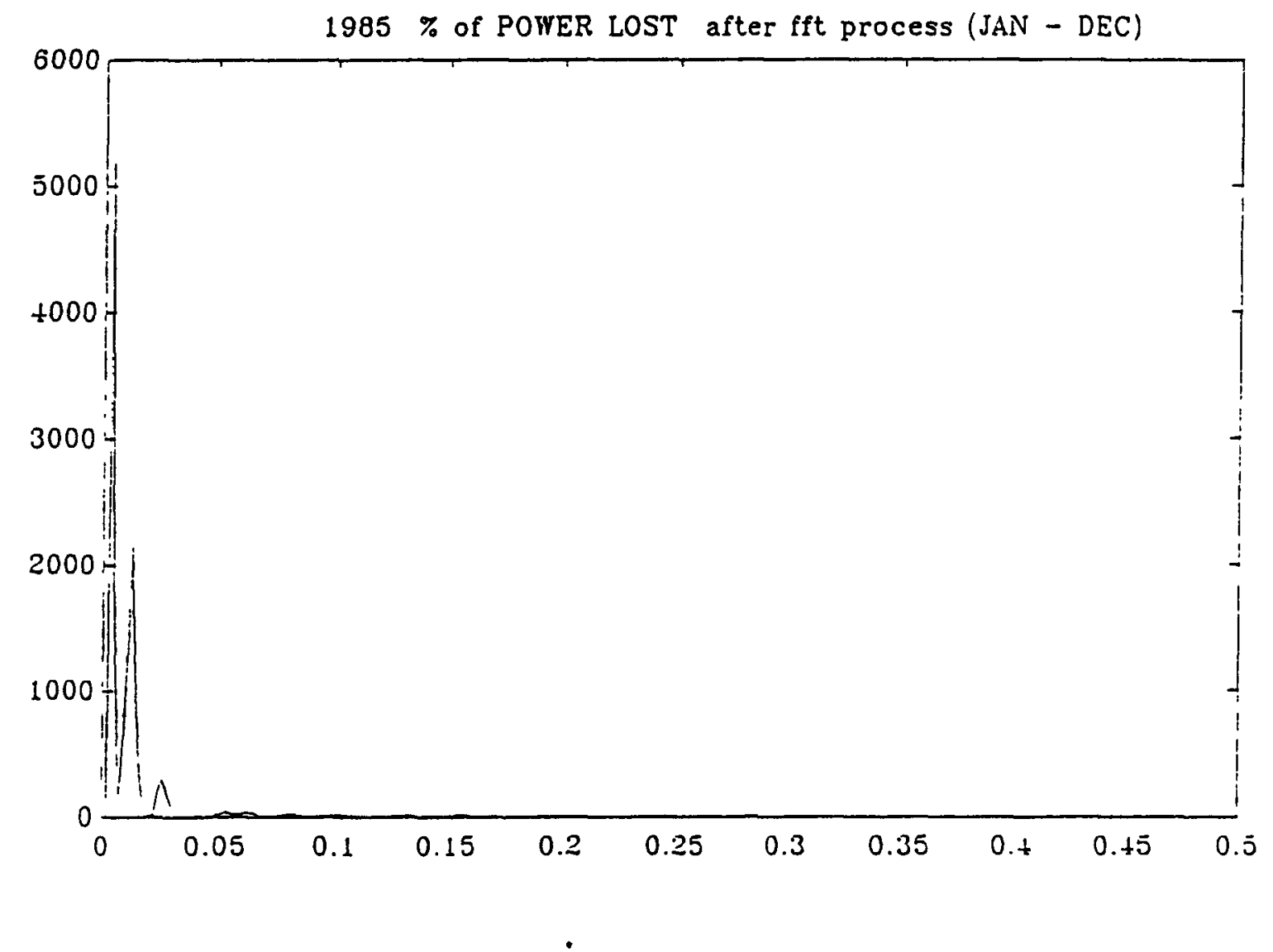

Att-1-49 


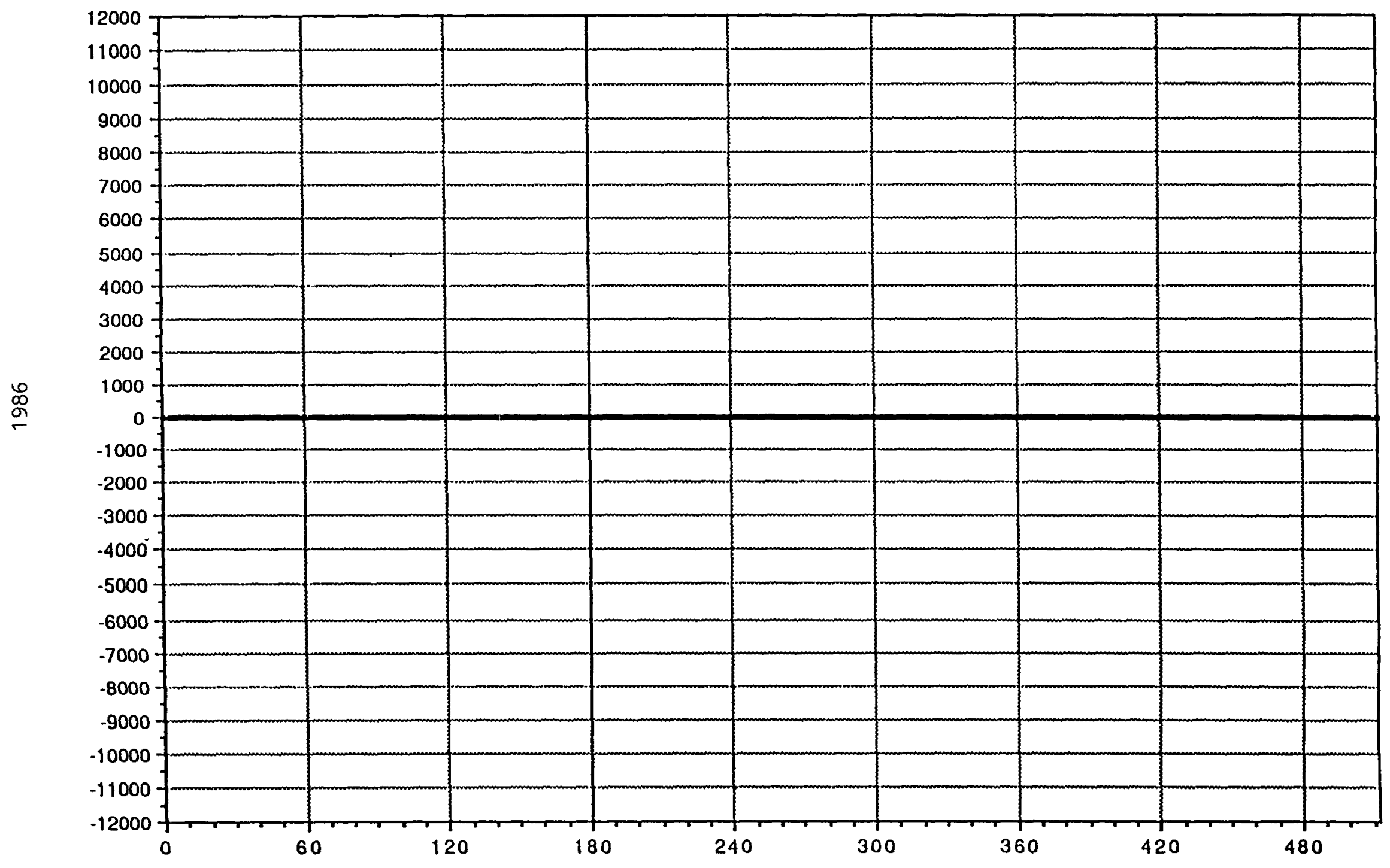




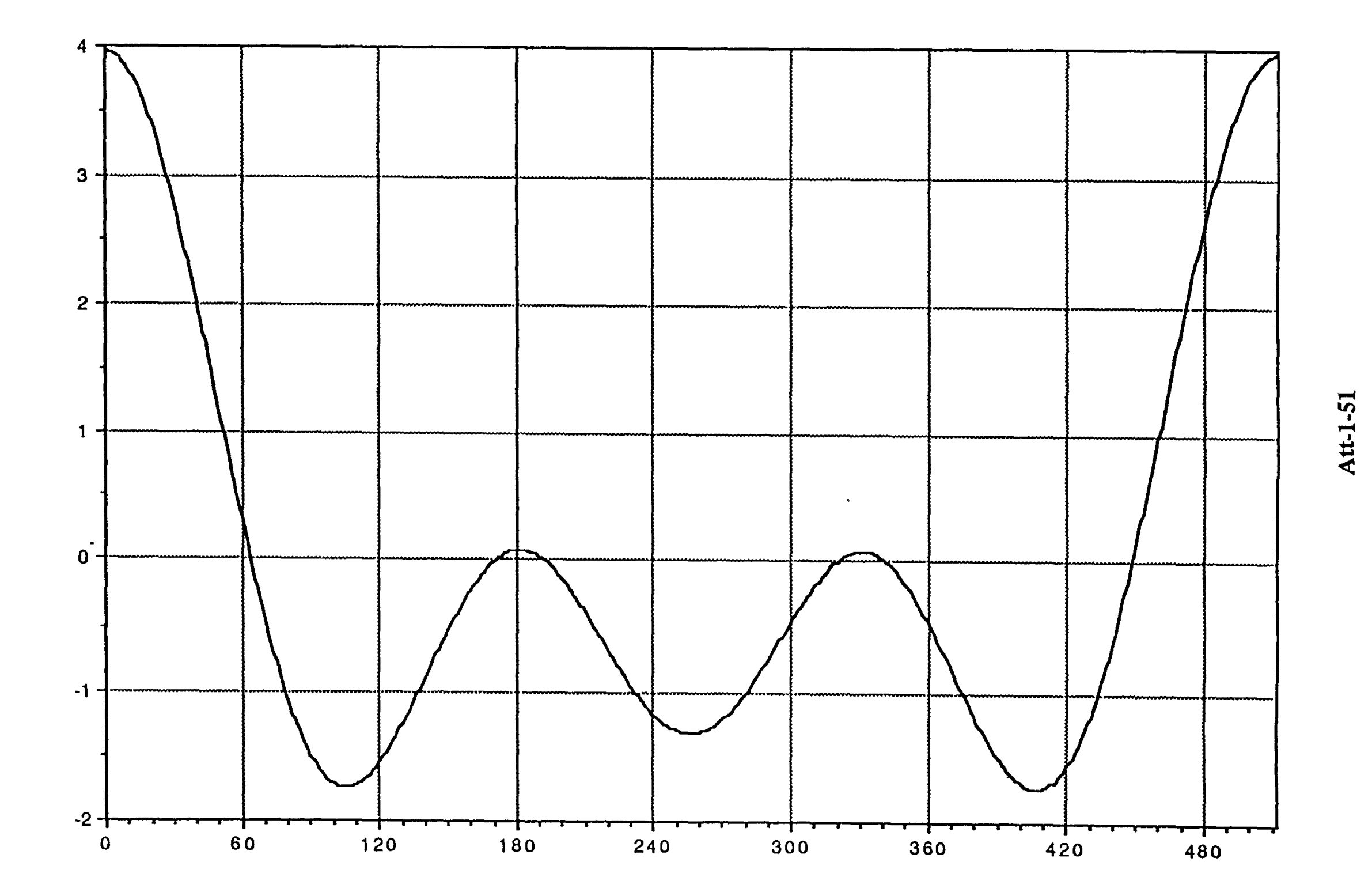




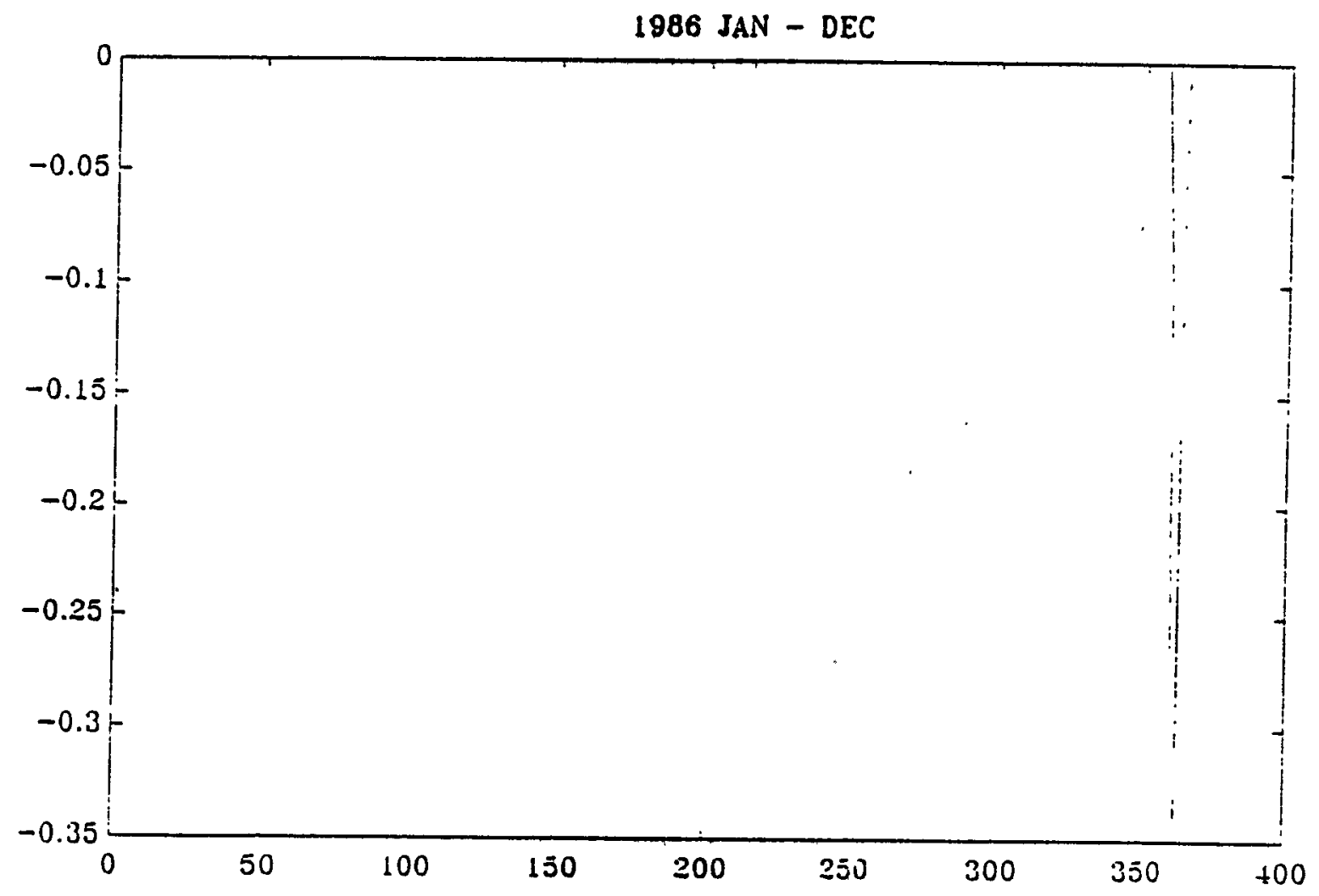




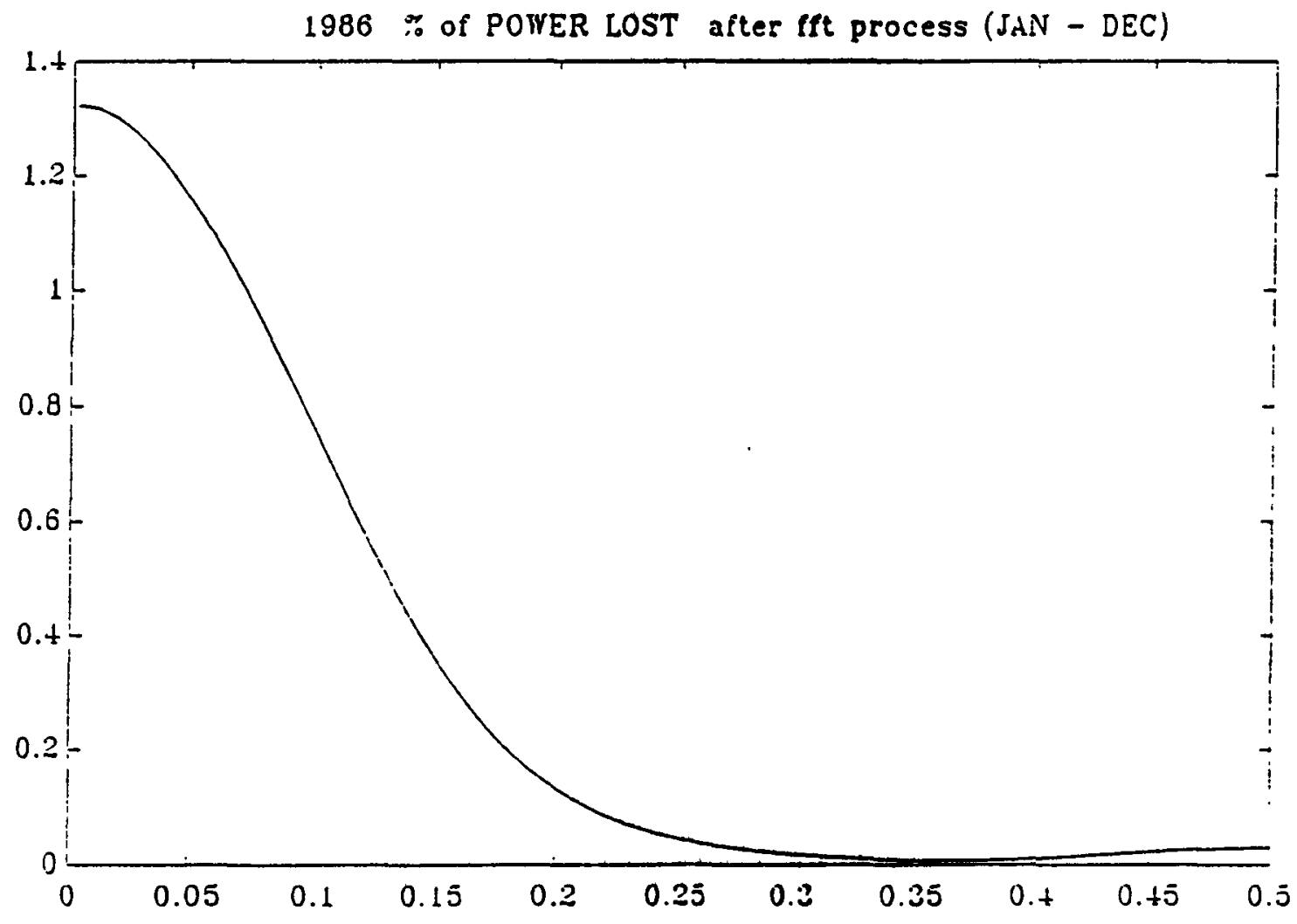




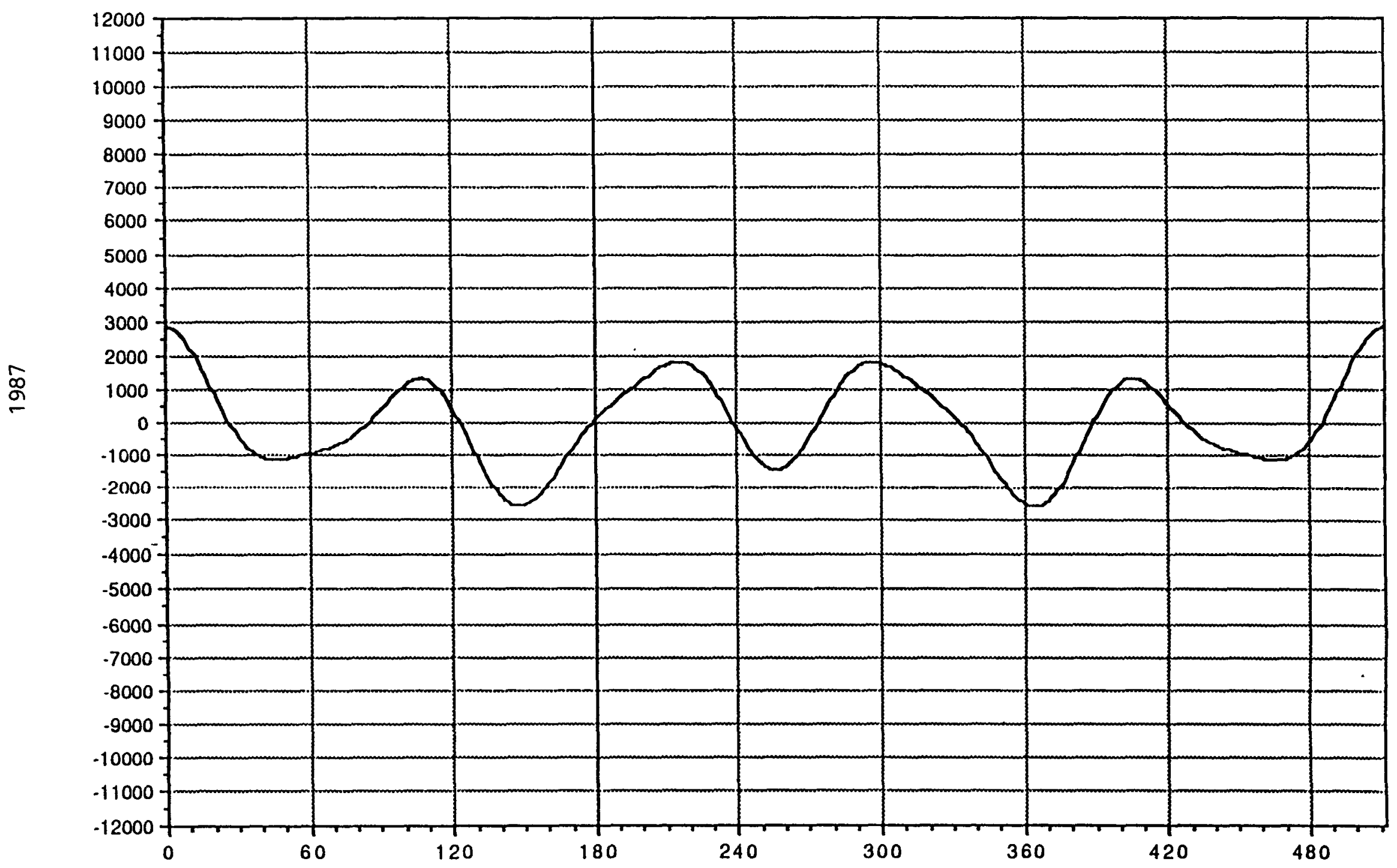




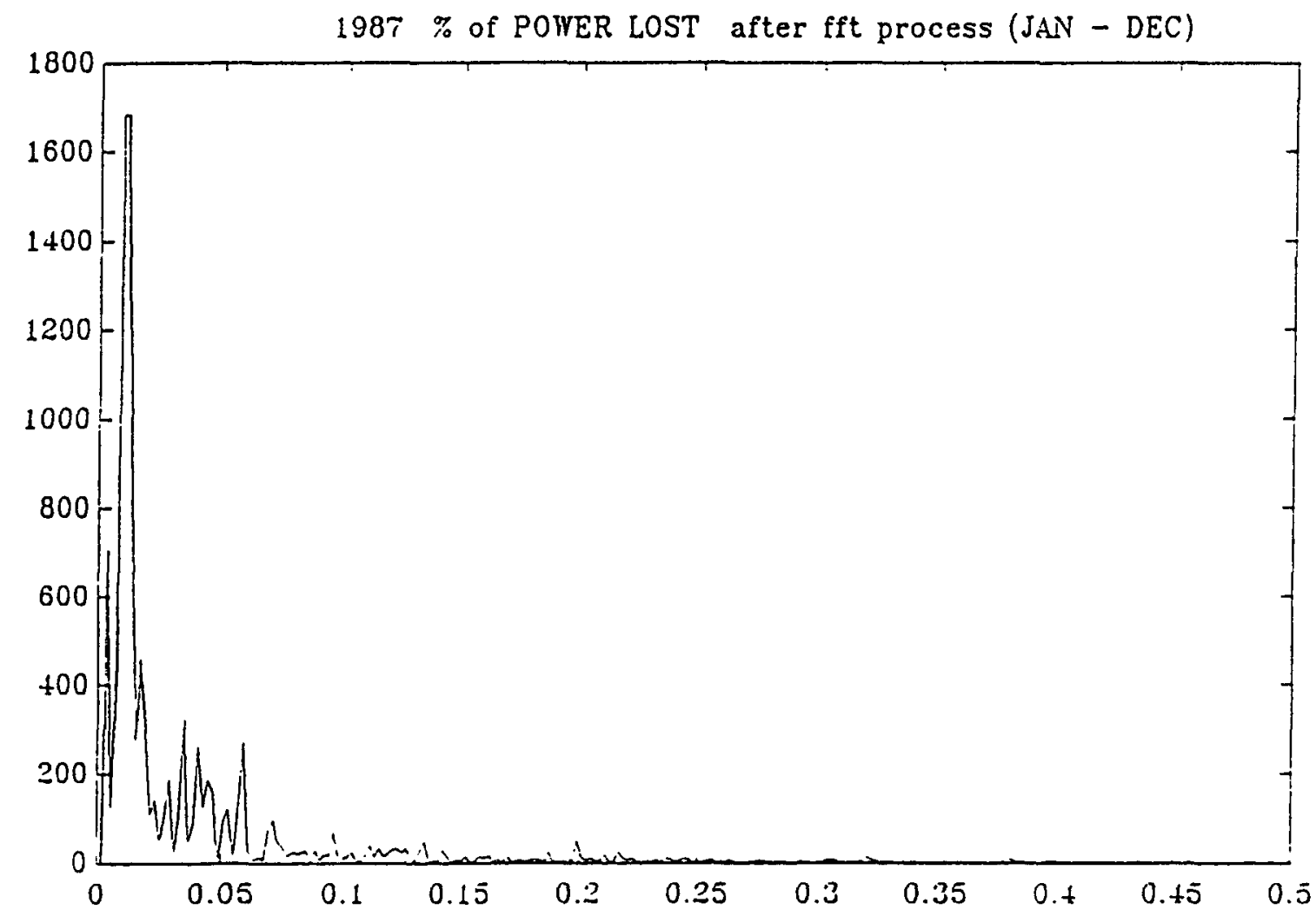

Att-1-55 


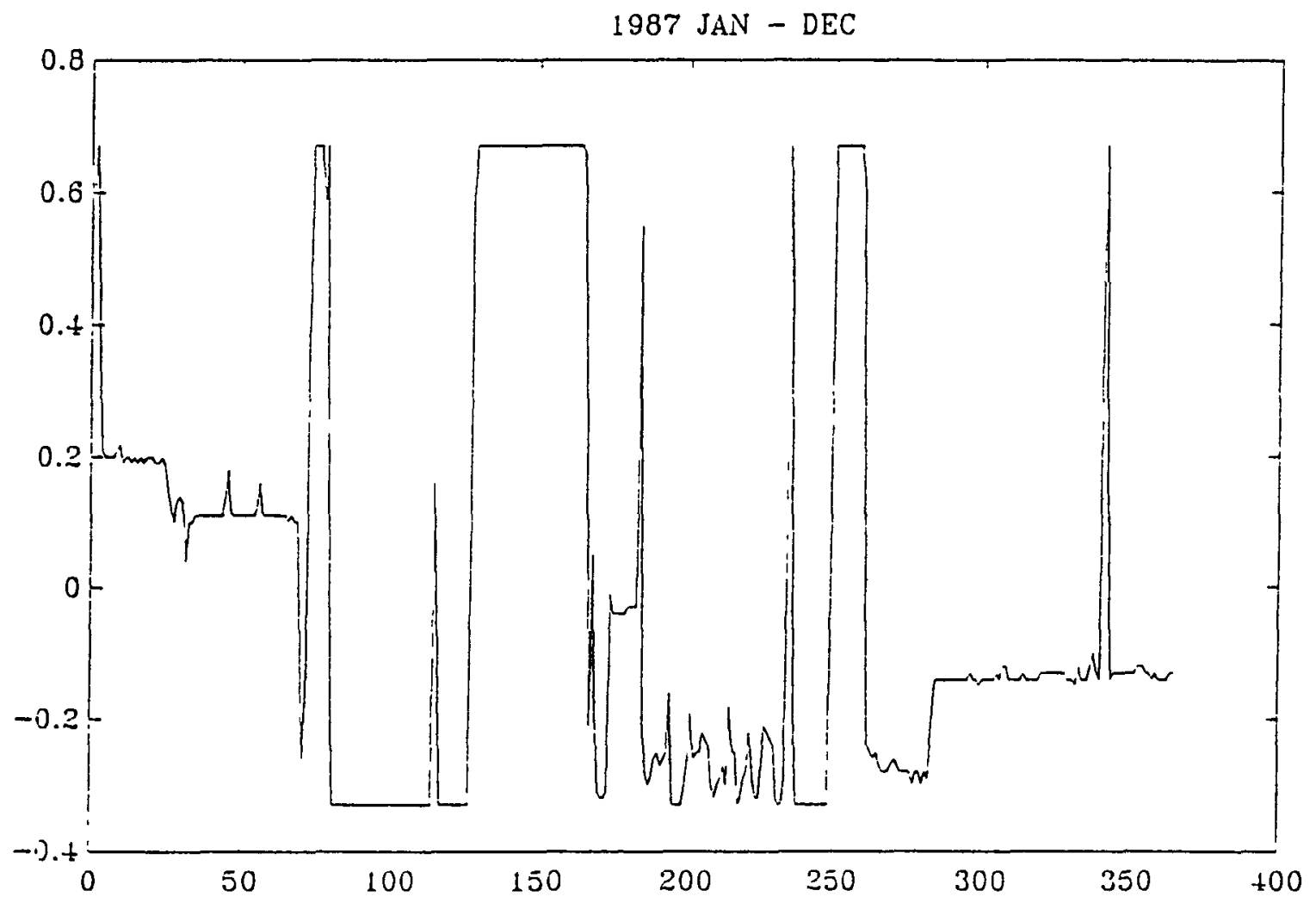

Att-1-56 


\section{Detailed Results for Plant V}

Att-1-57 


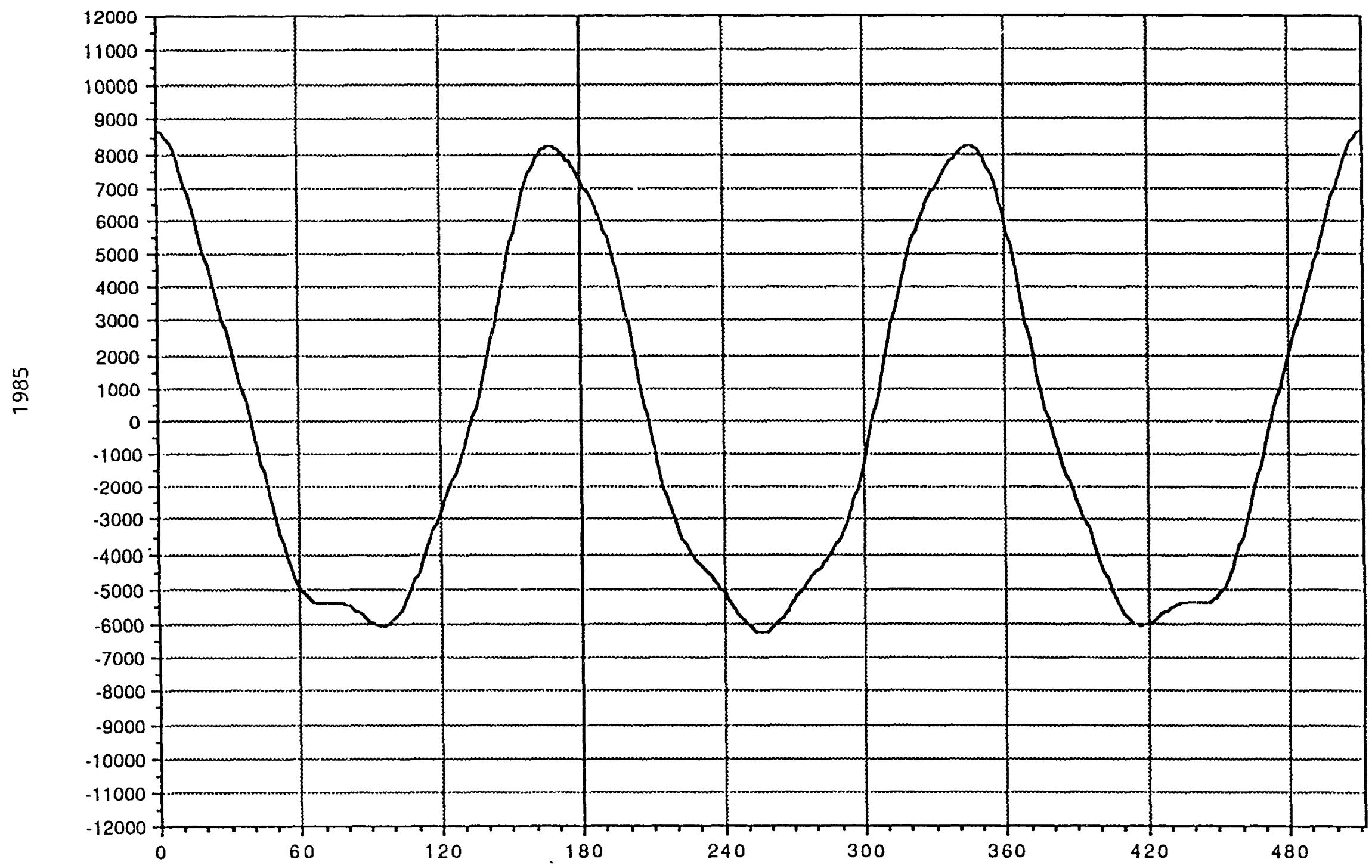


1985 JAN - DEC



Att-1-59 


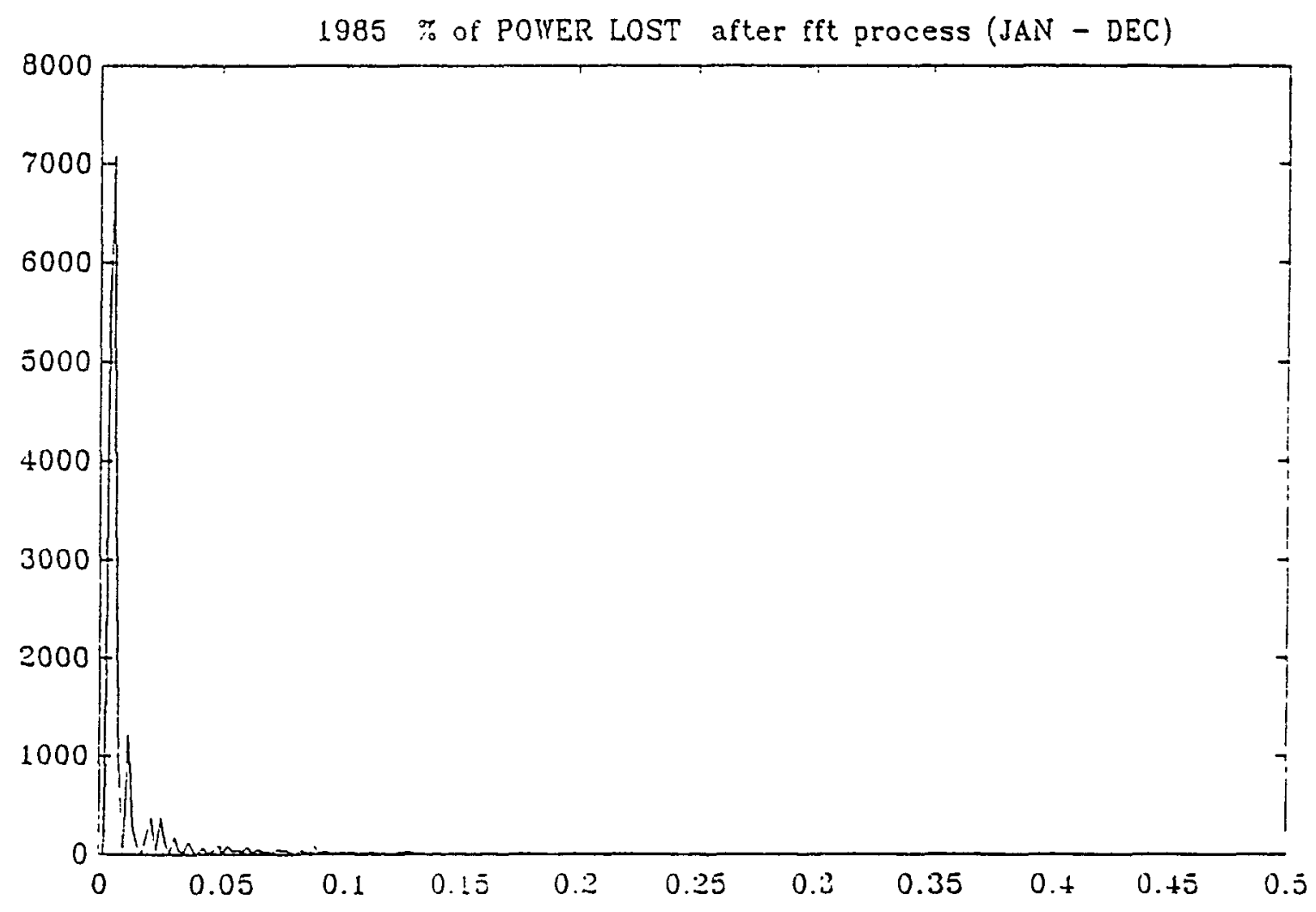




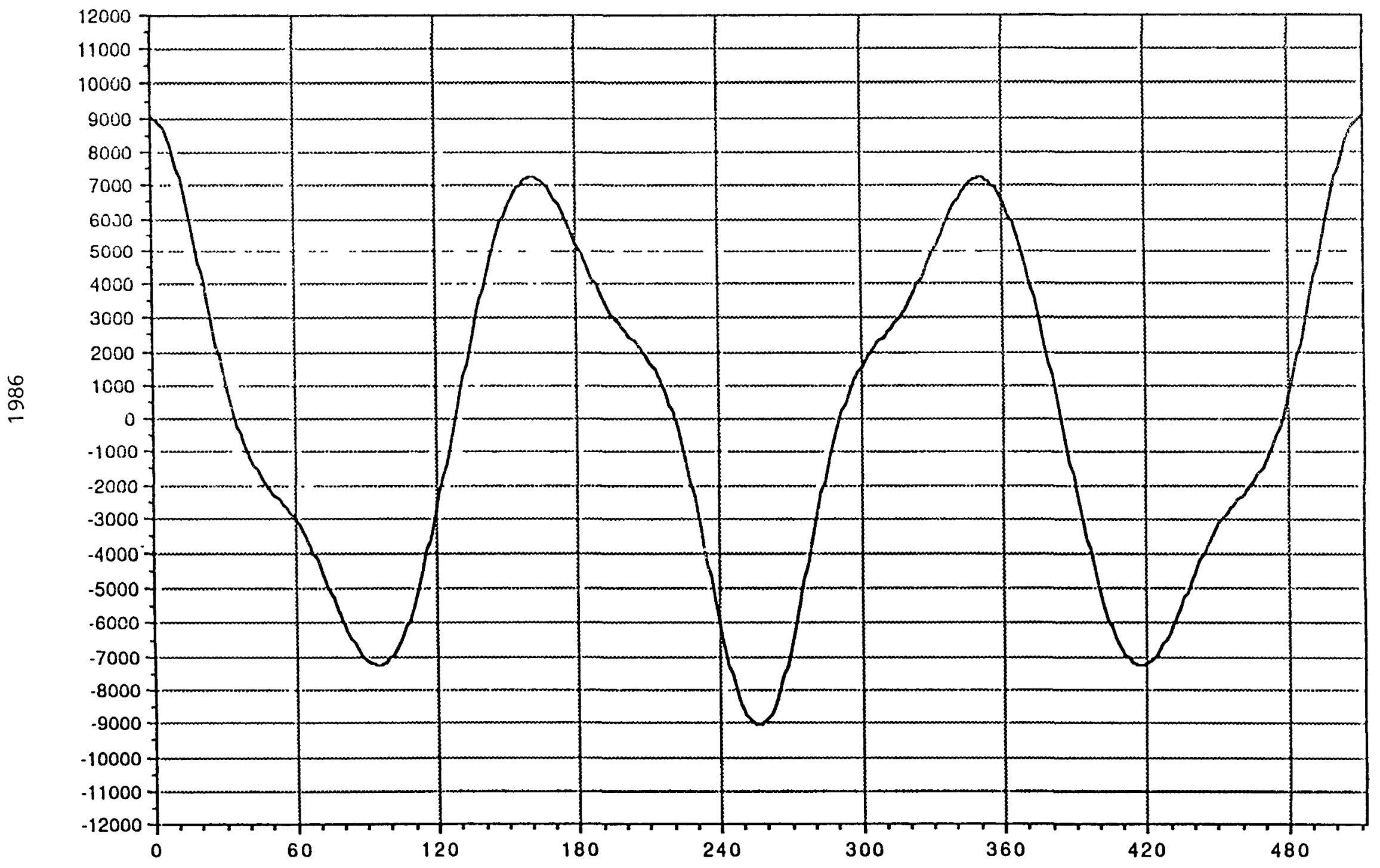




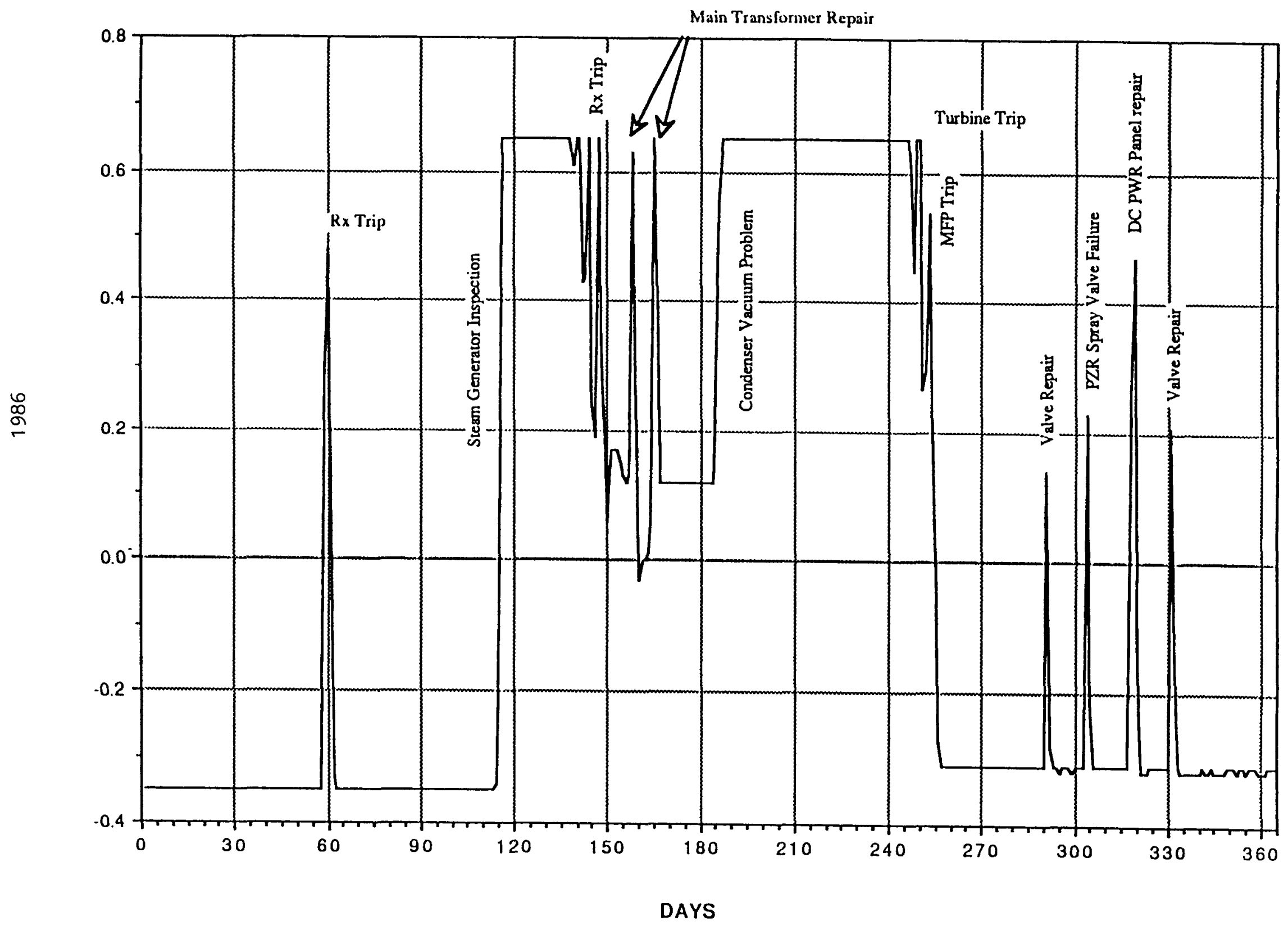

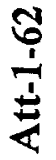




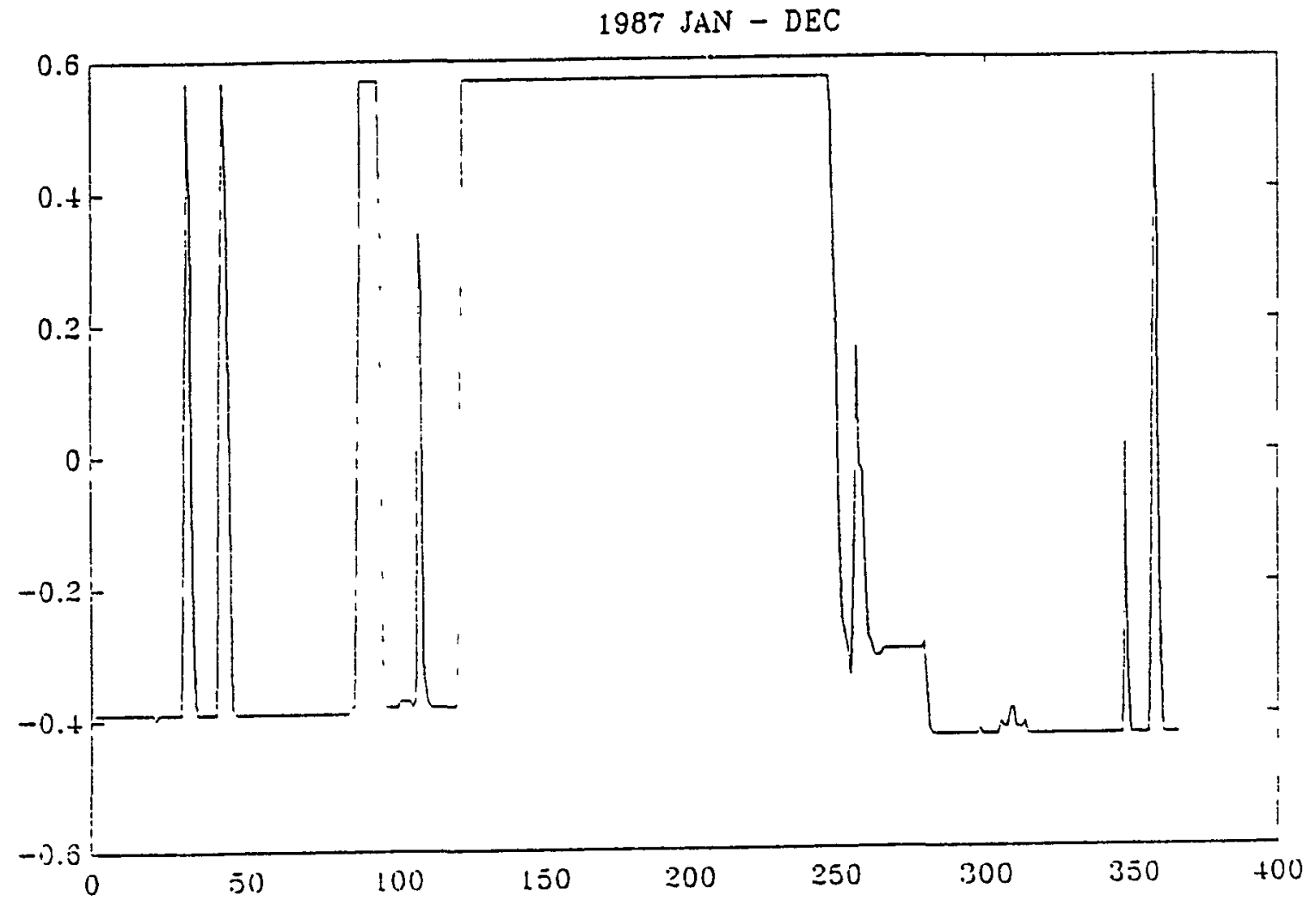




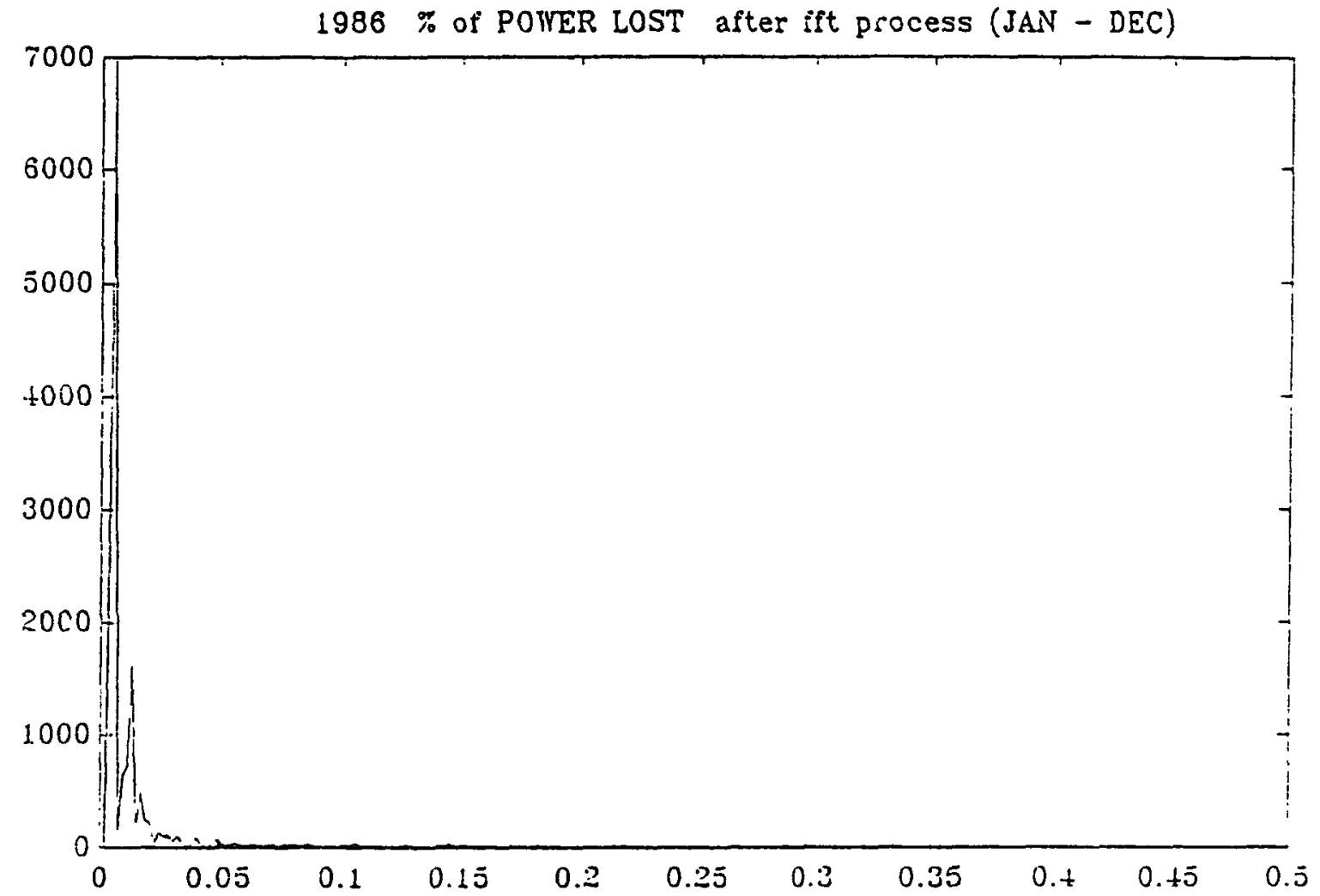




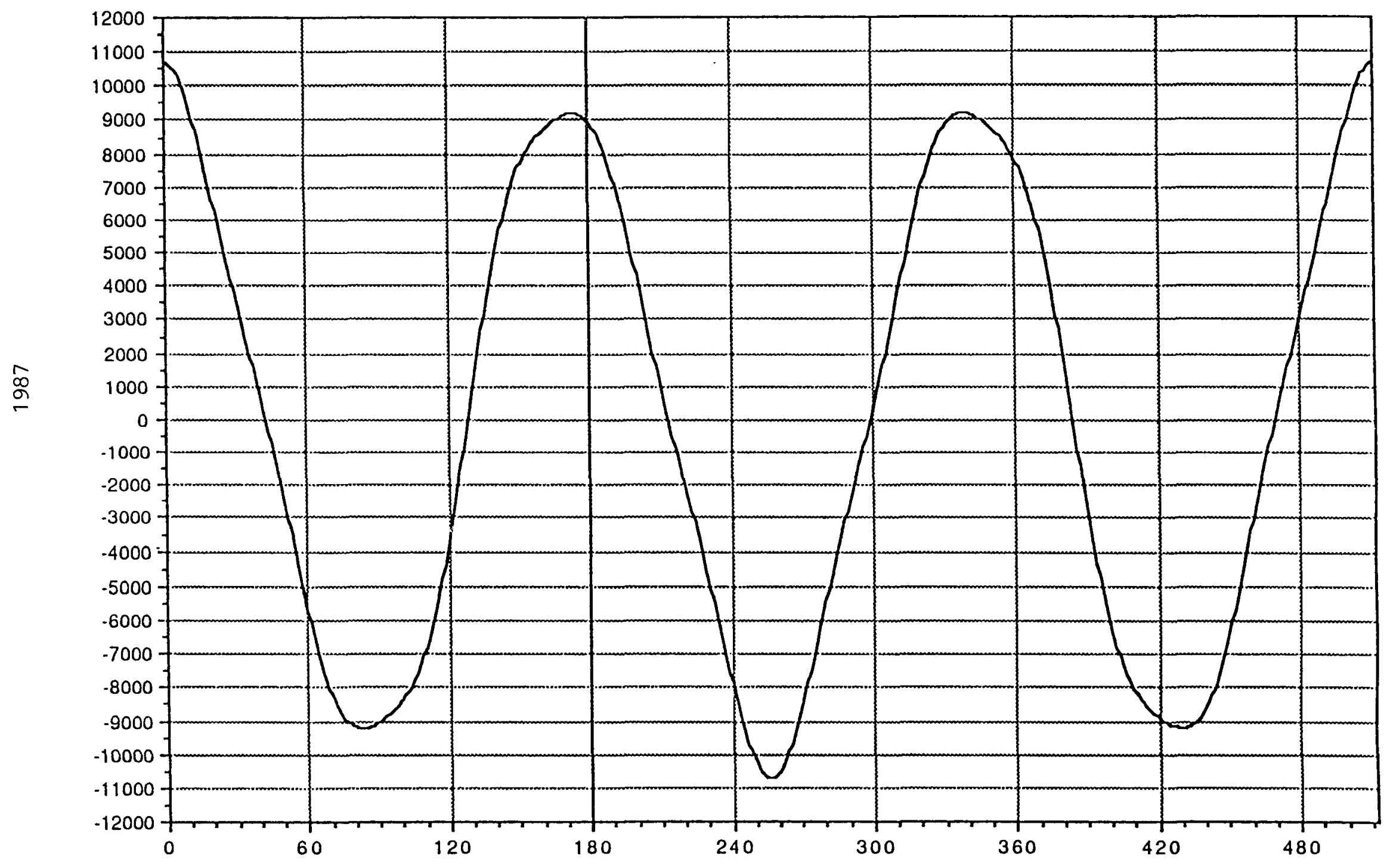

Row Numbers 


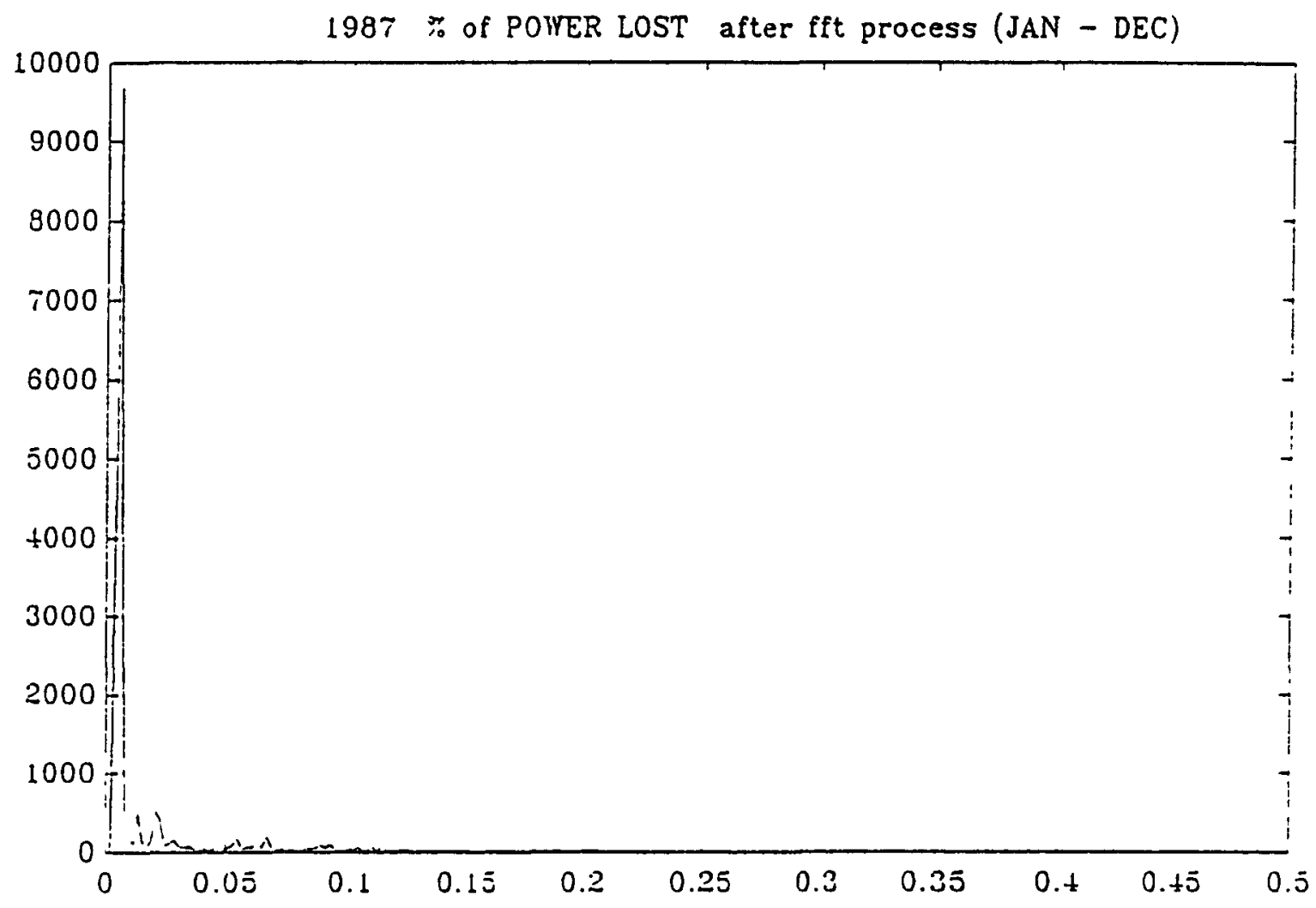


Detailed Results for Plant W 


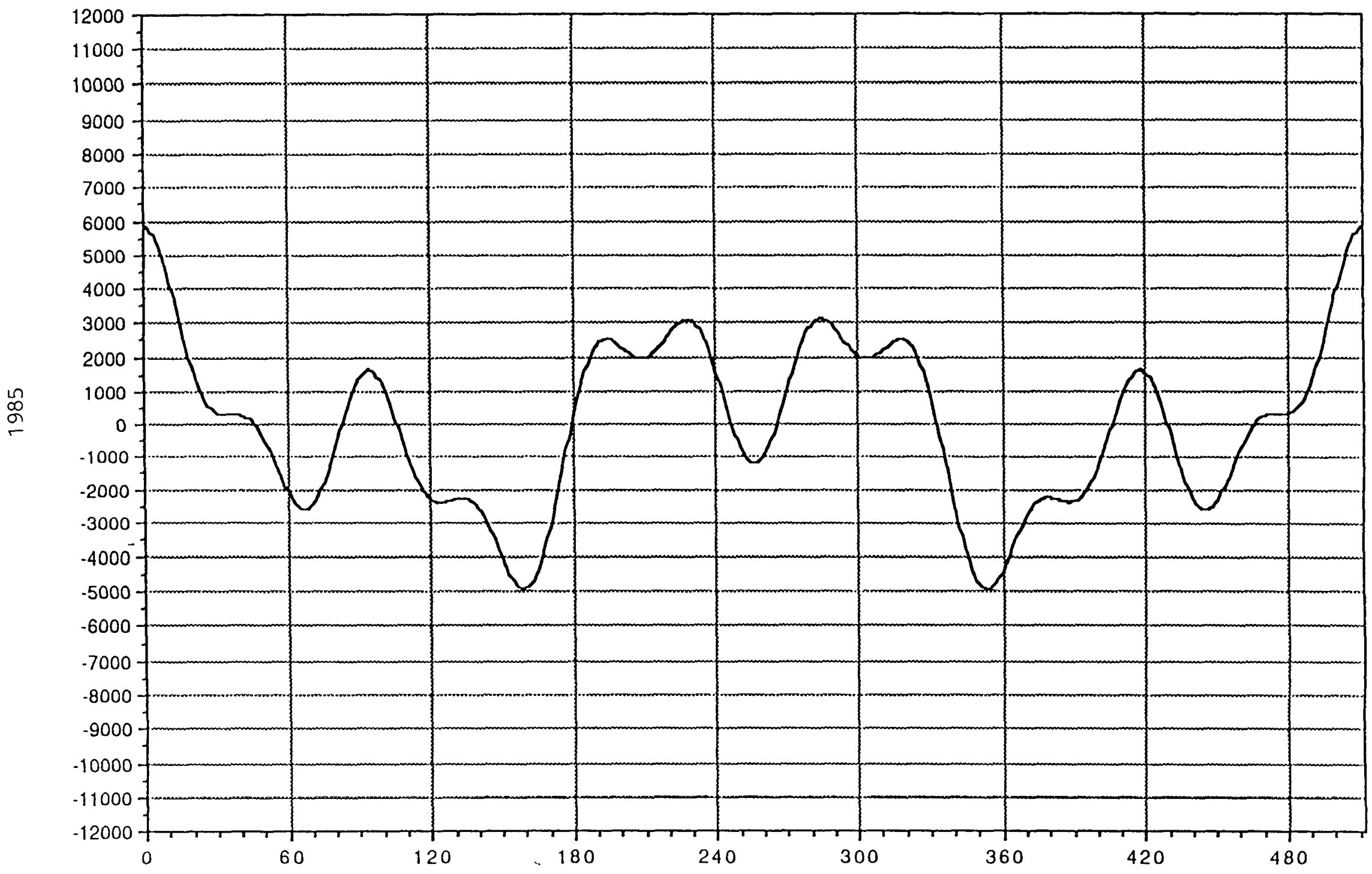




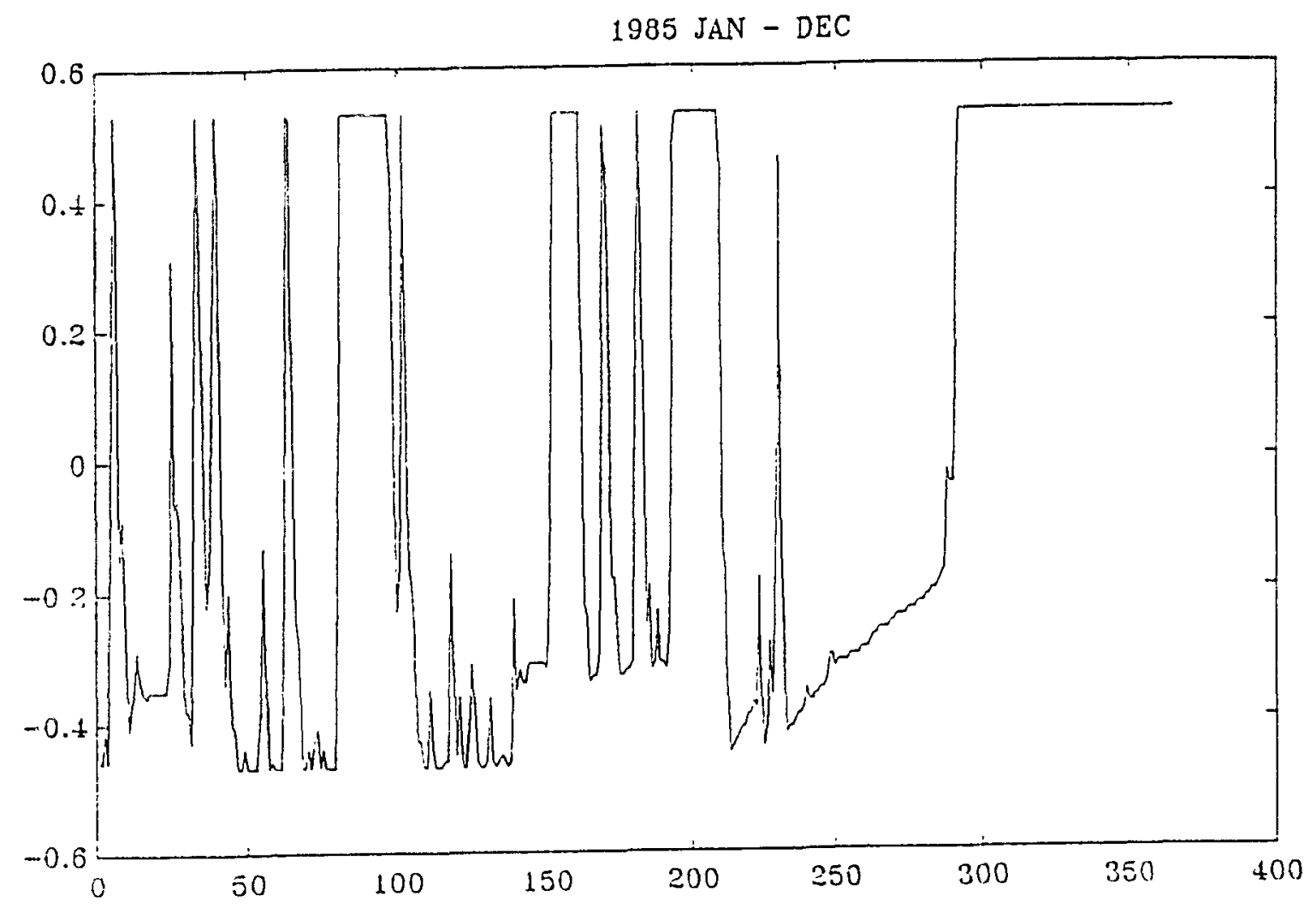




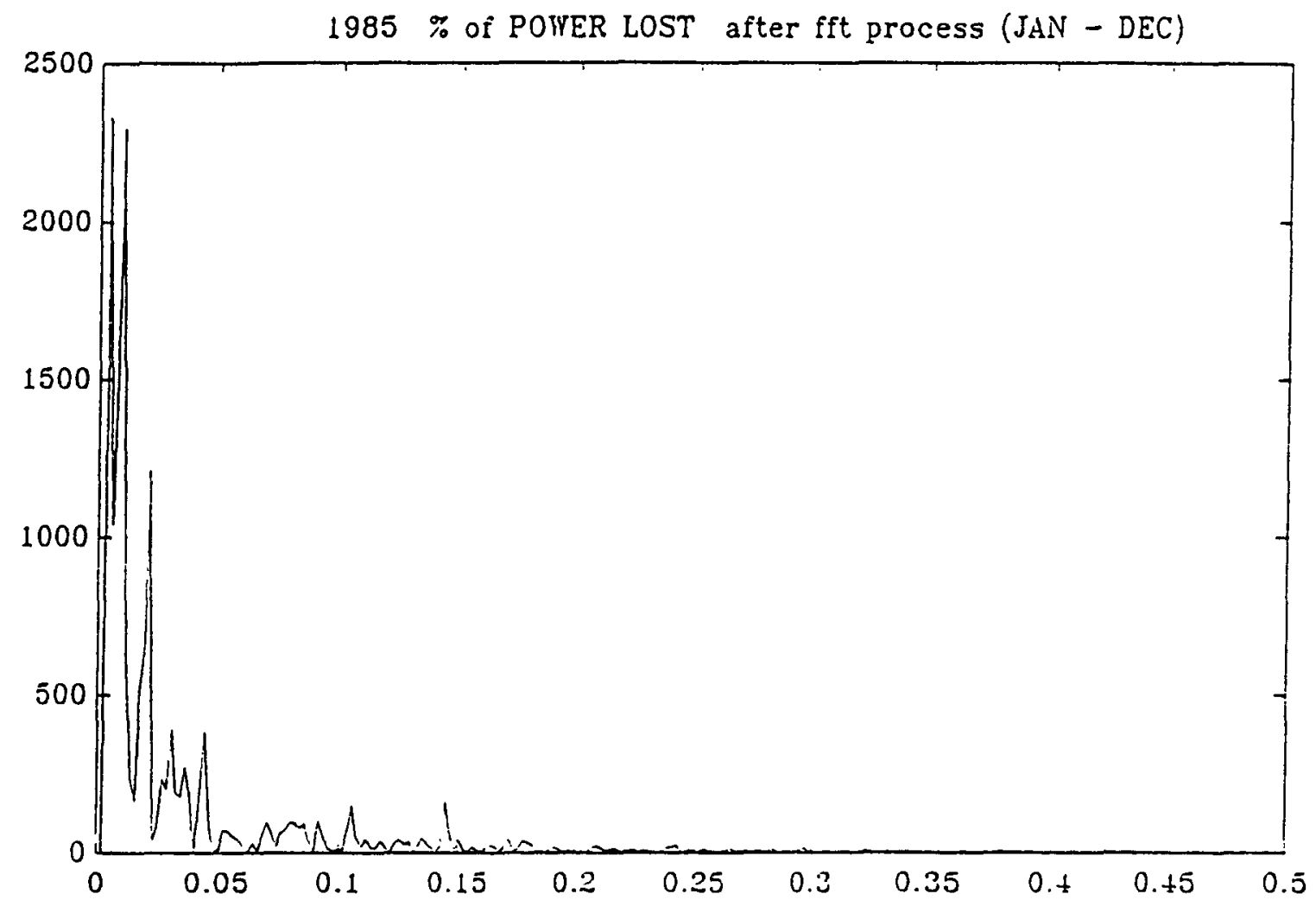




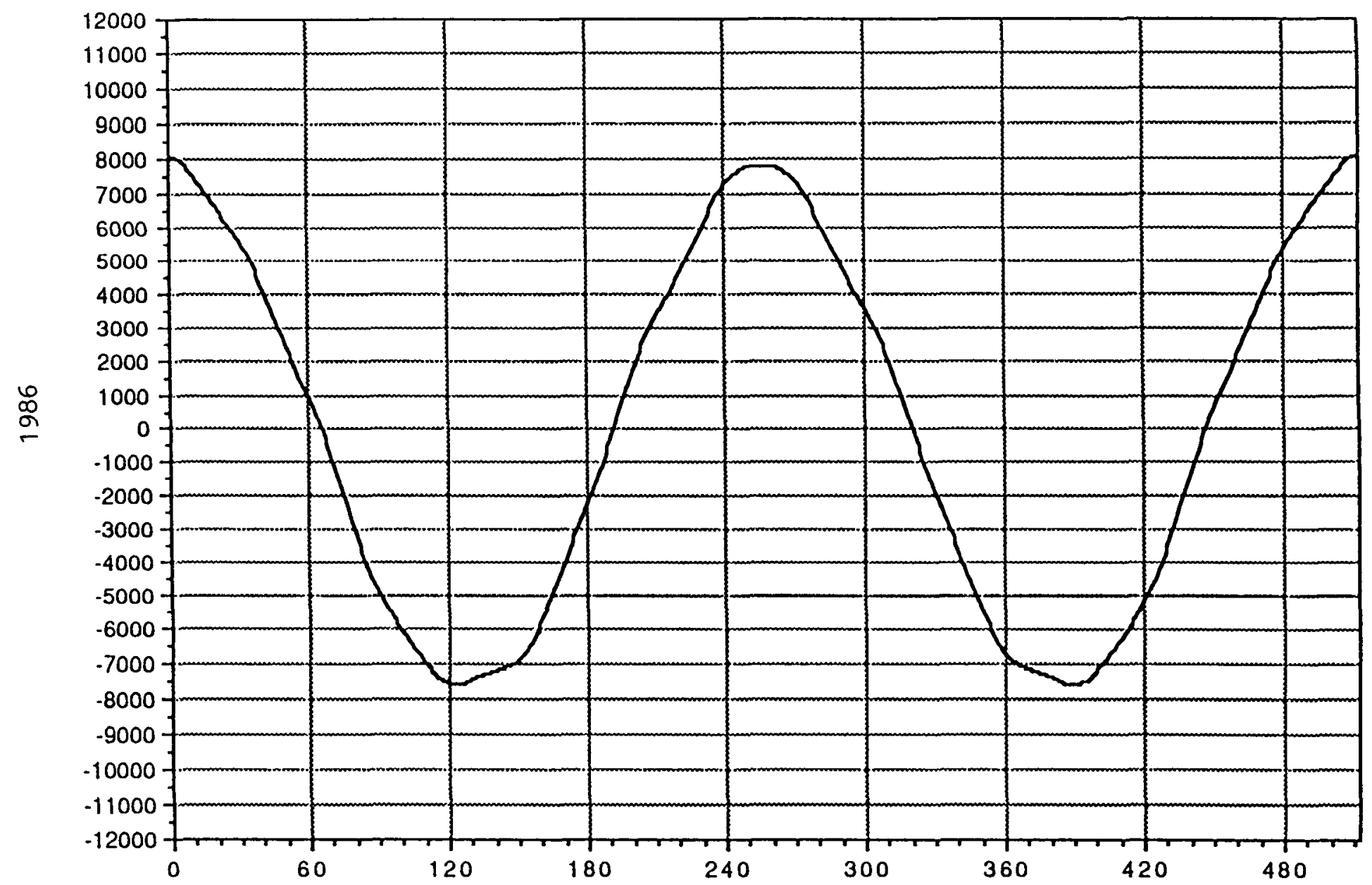




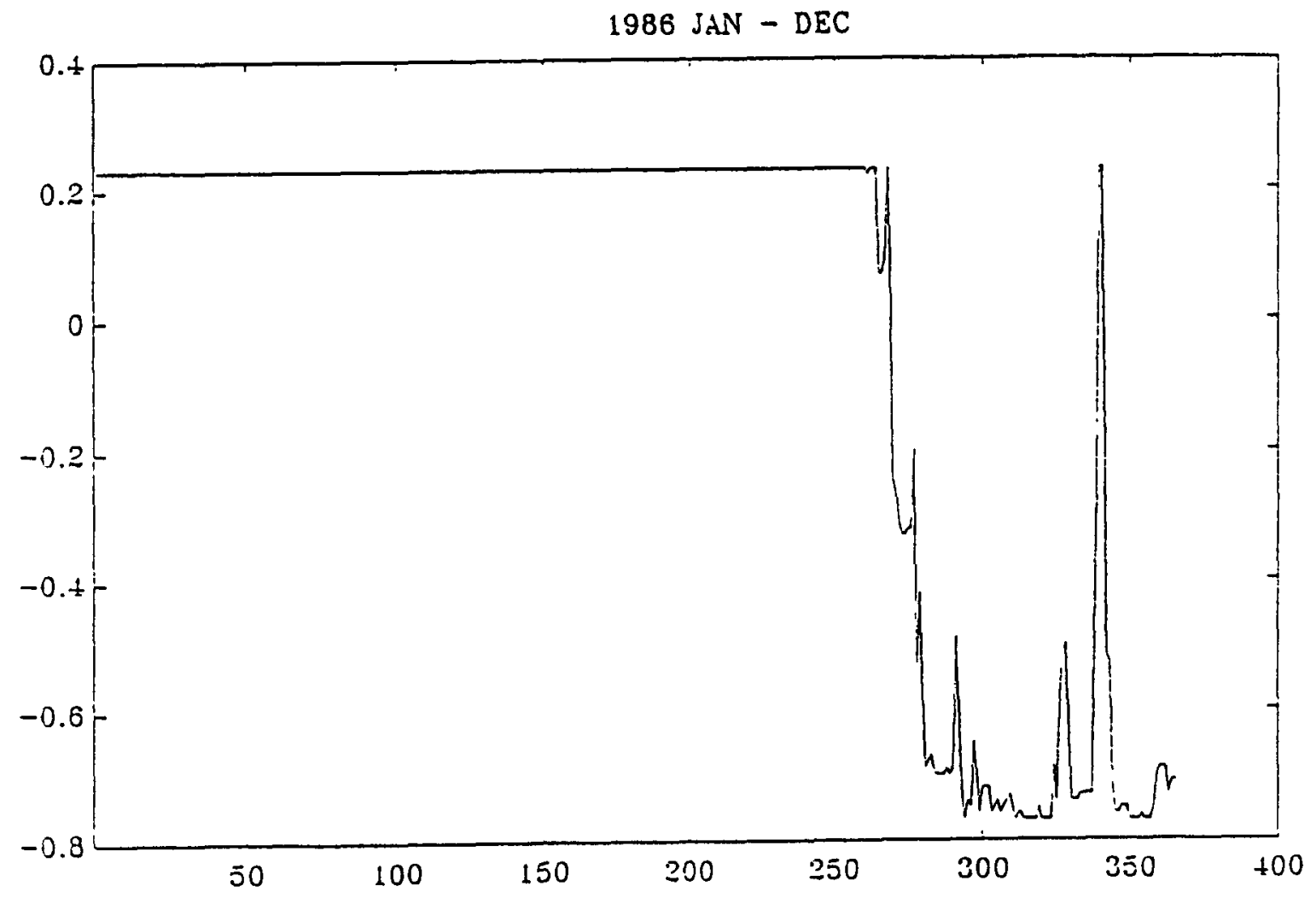

Att-1-72 


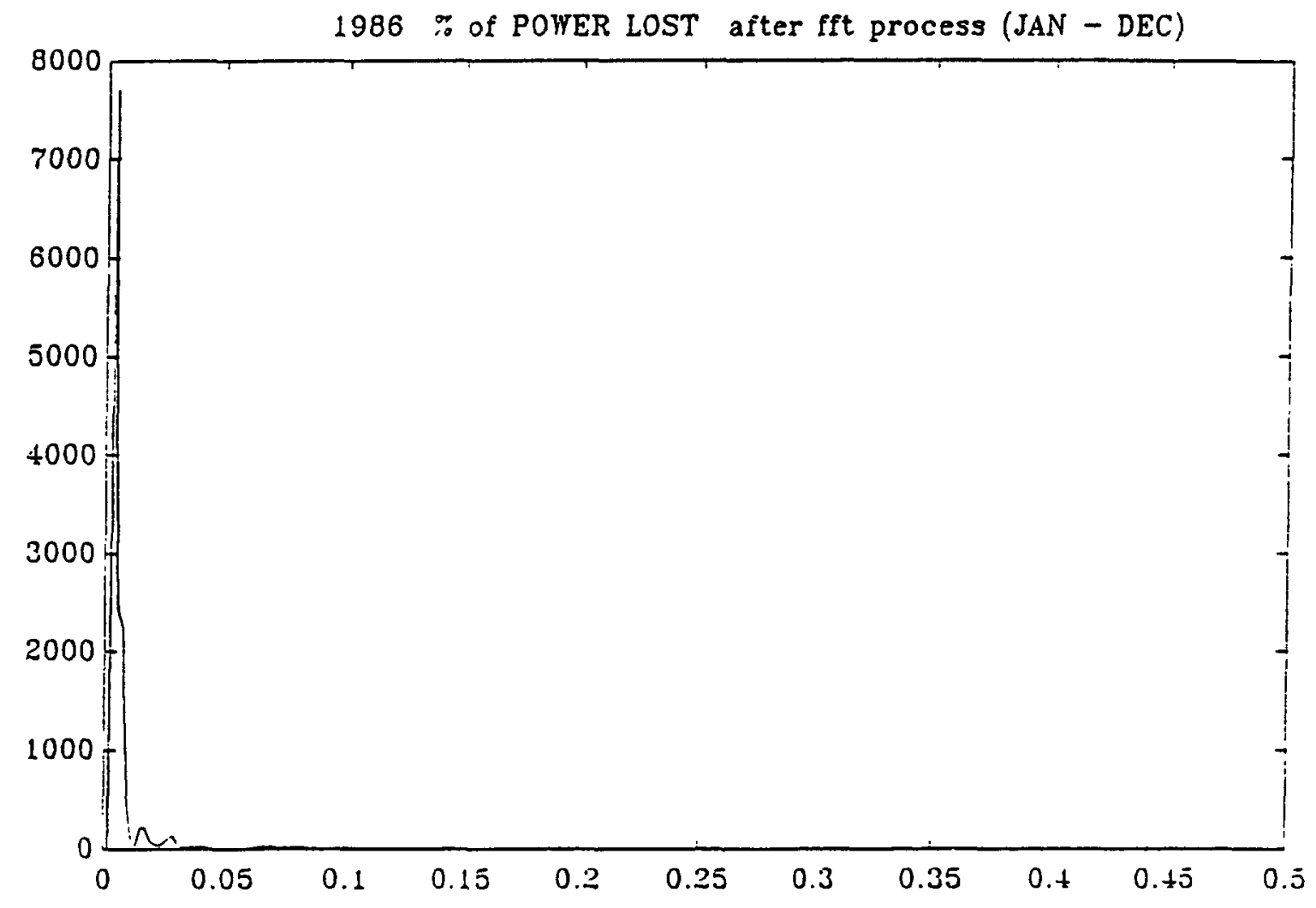

Att-1-73 


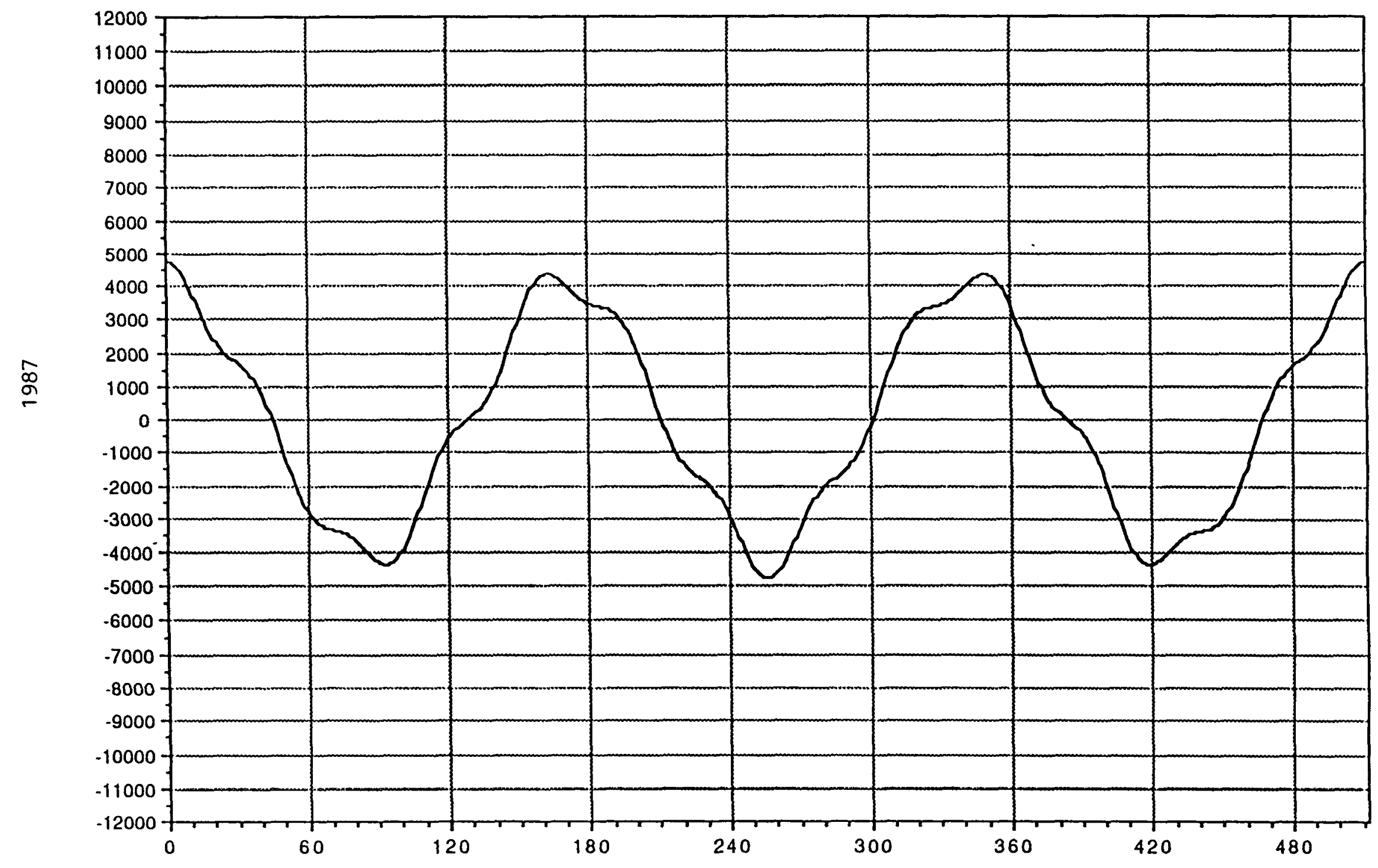




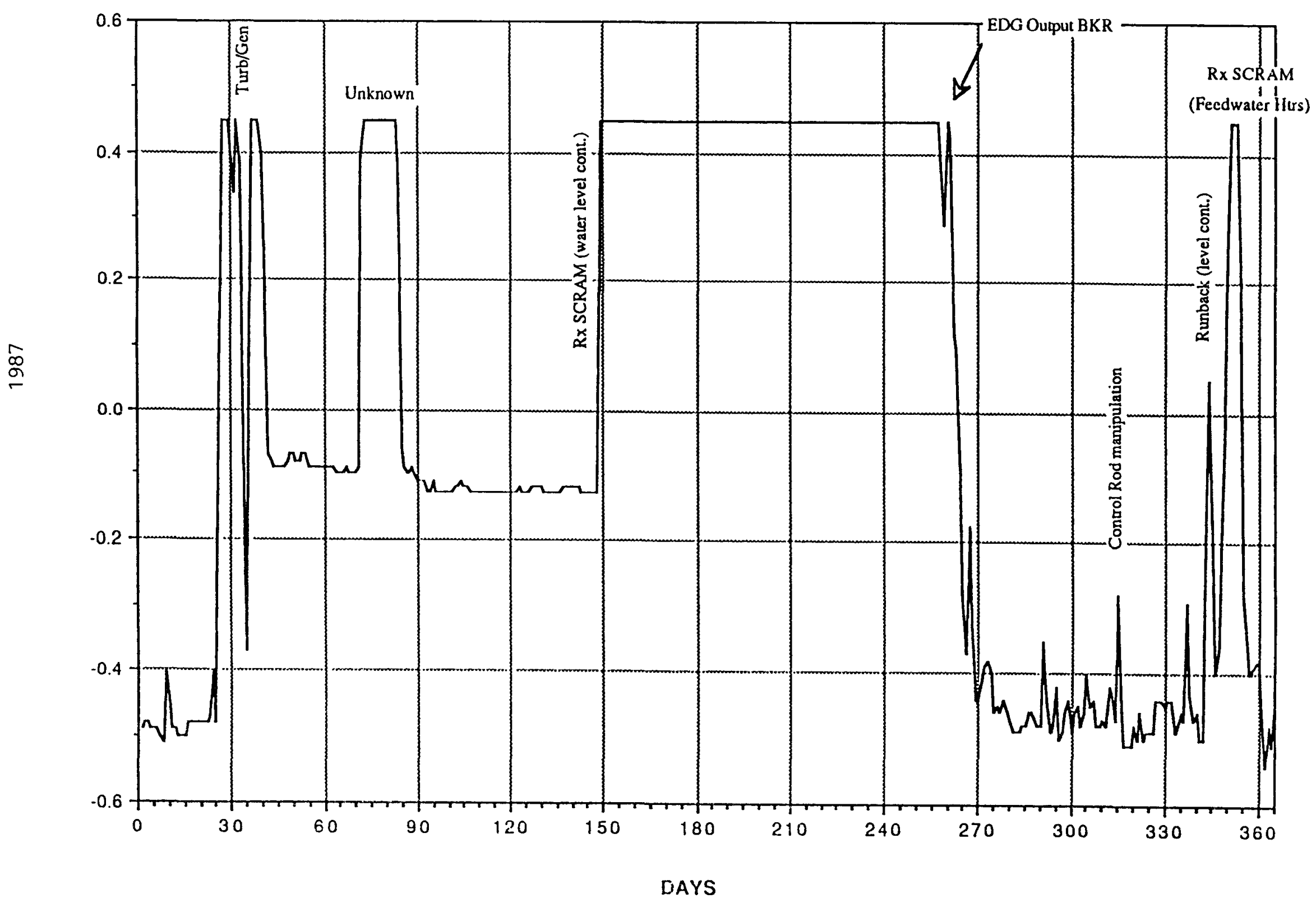

$\frac{1}{3}$ 


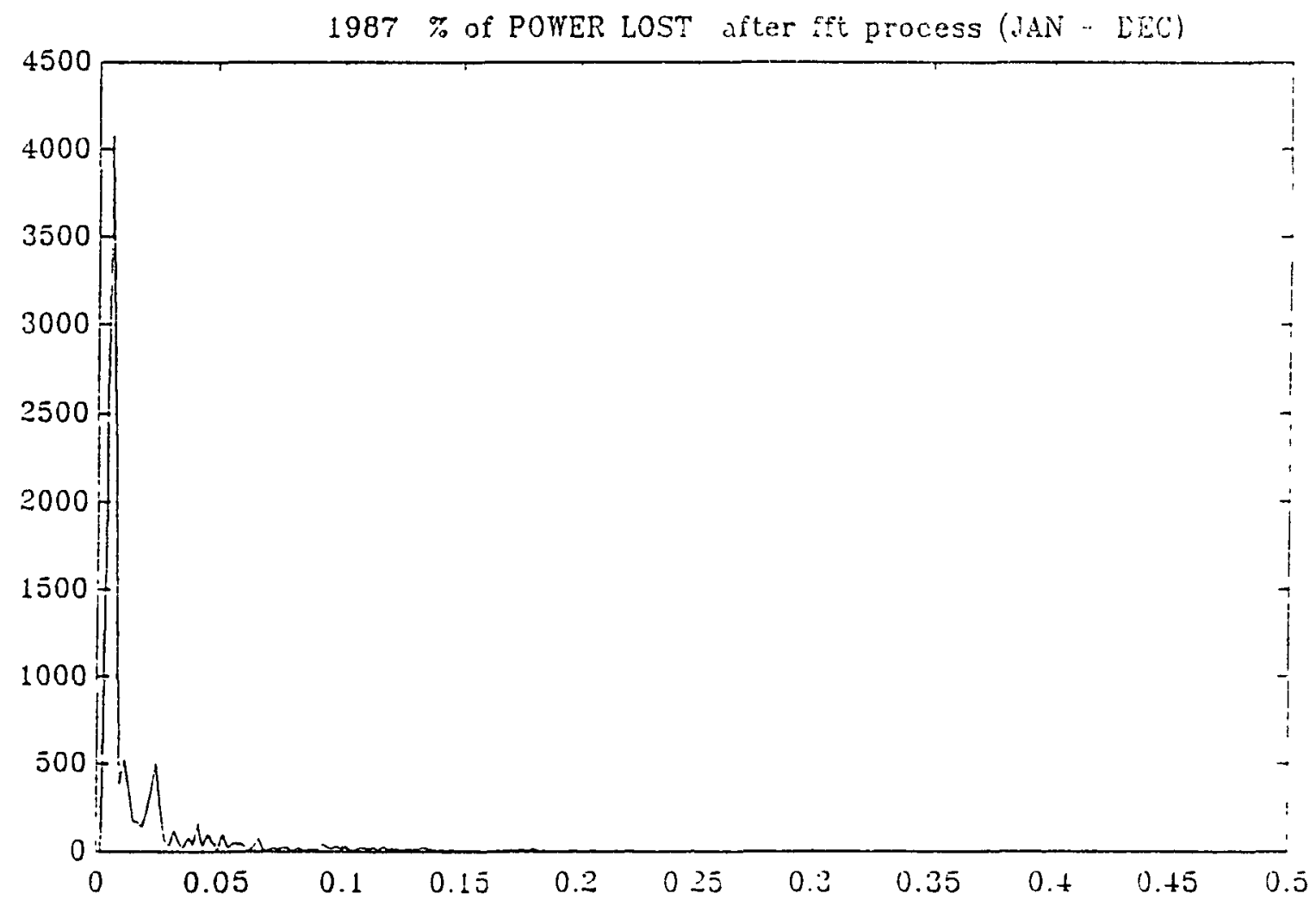

Att-1-76 


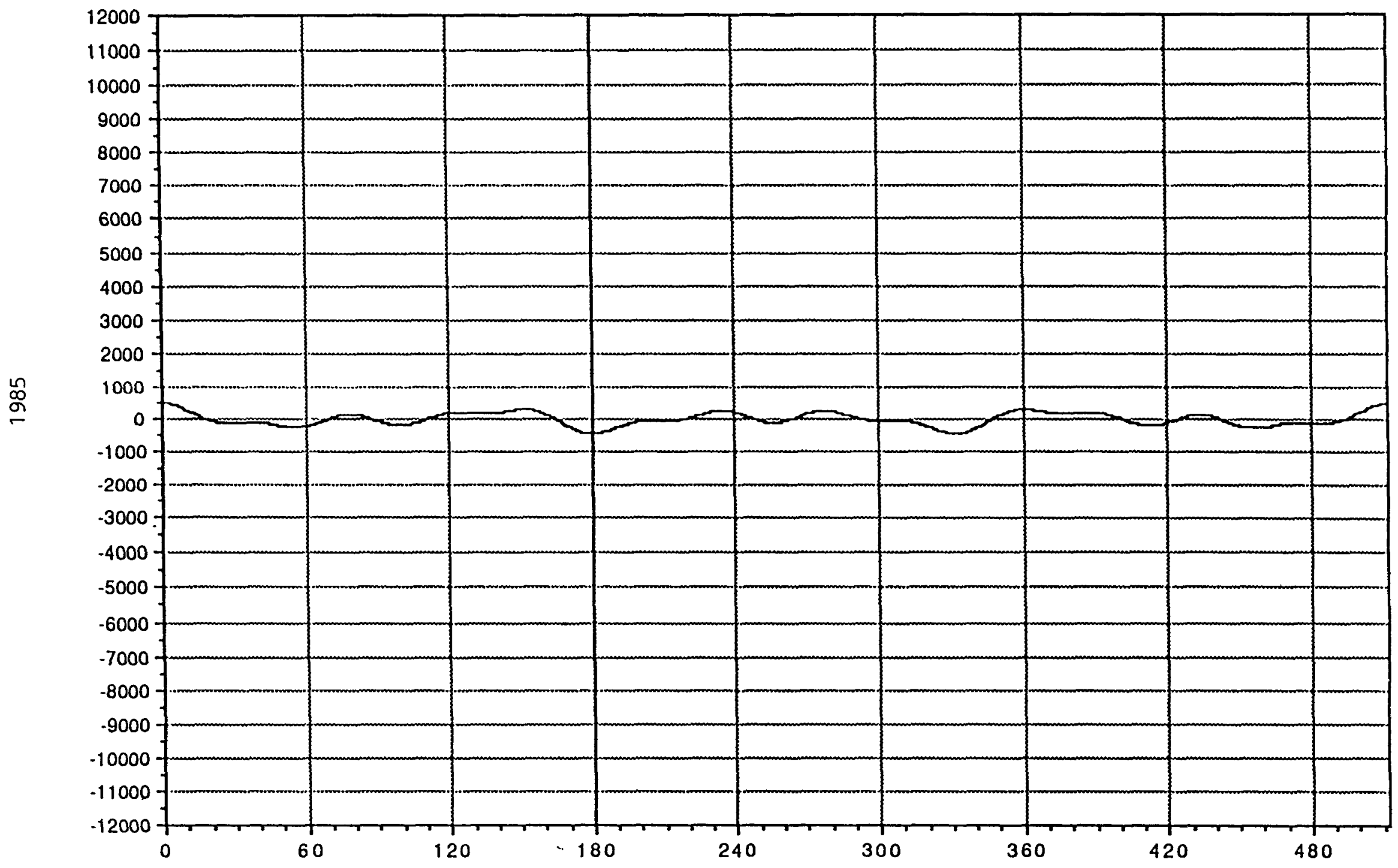




\section{Detailed Results for Plant X}




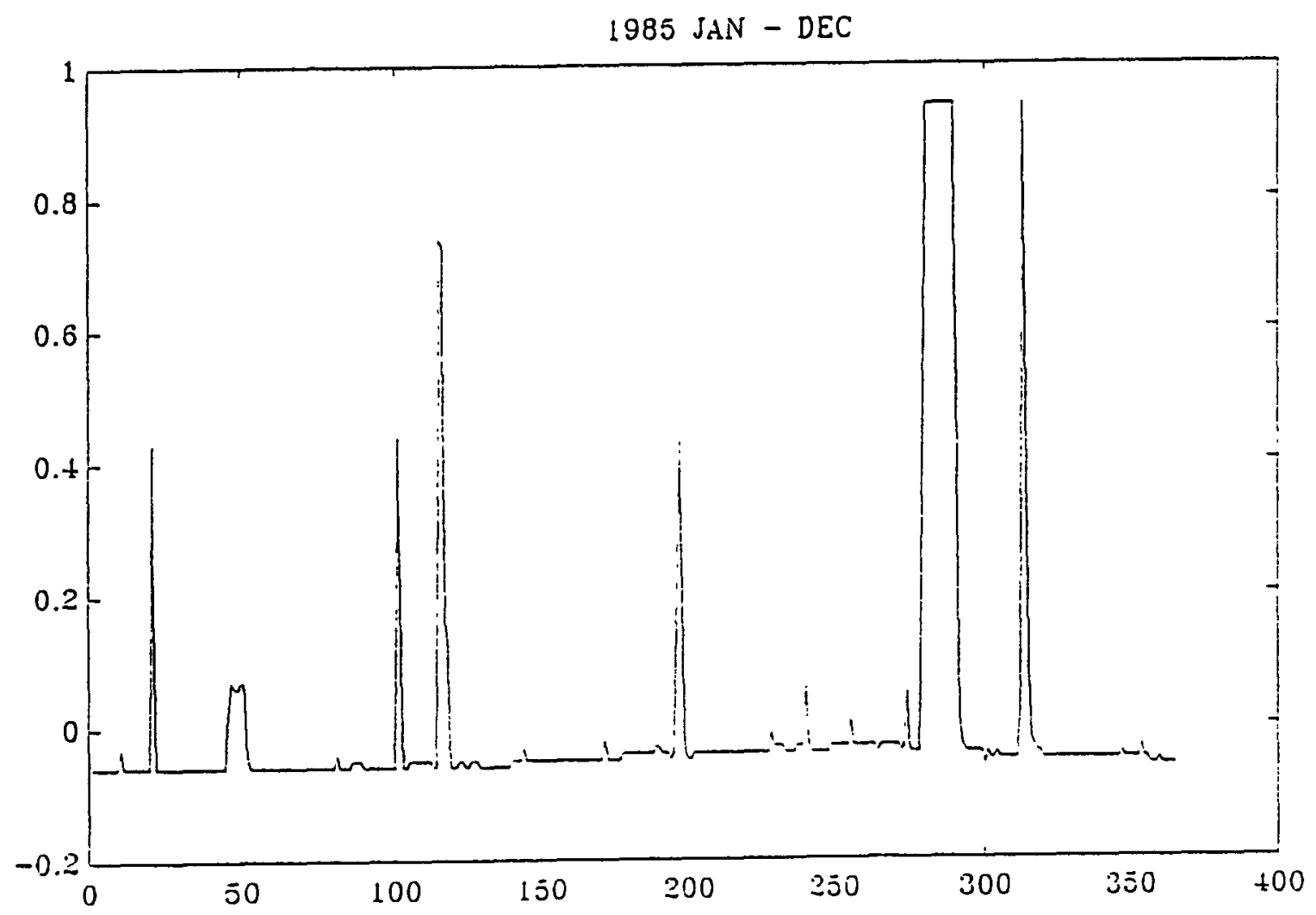




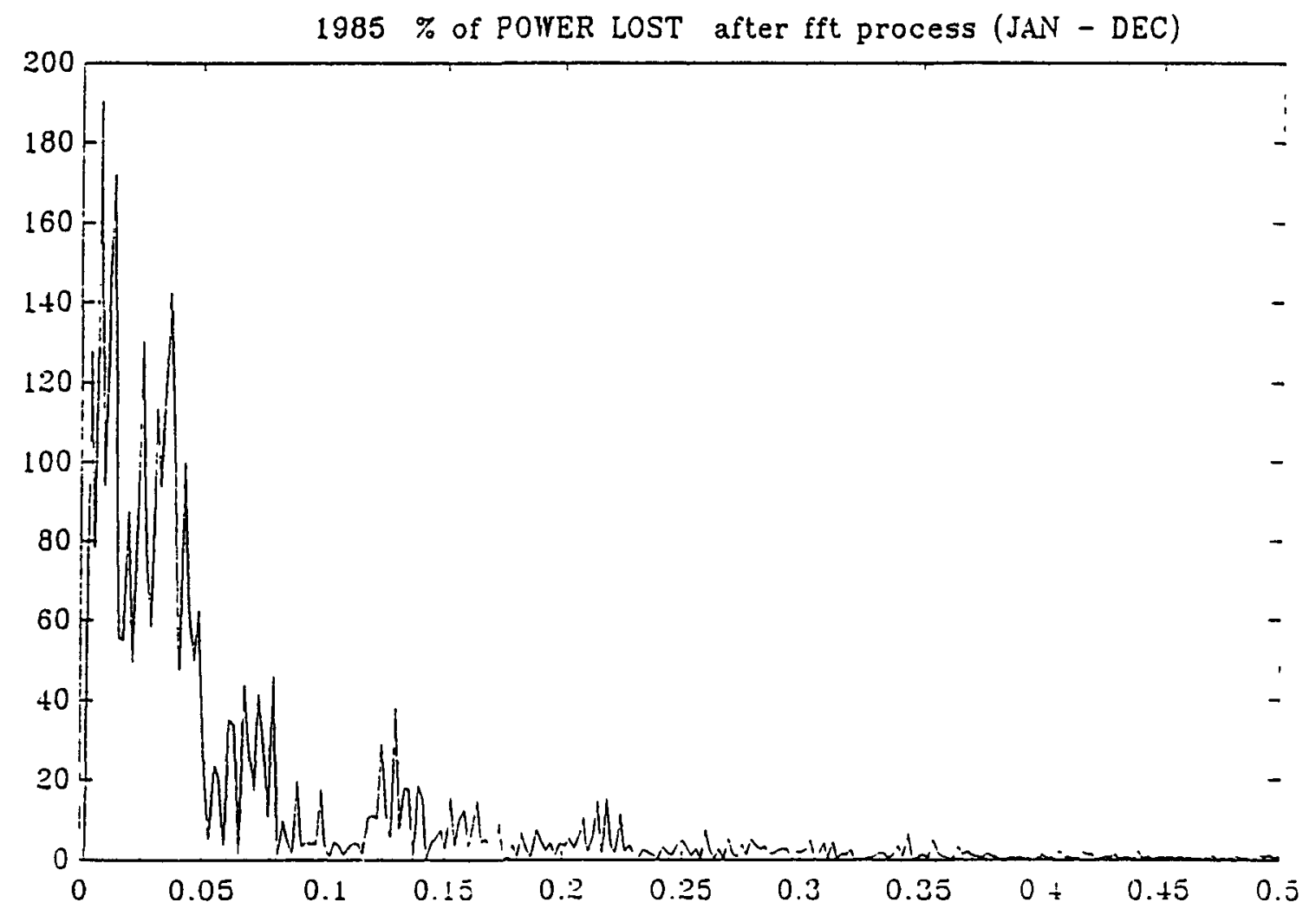




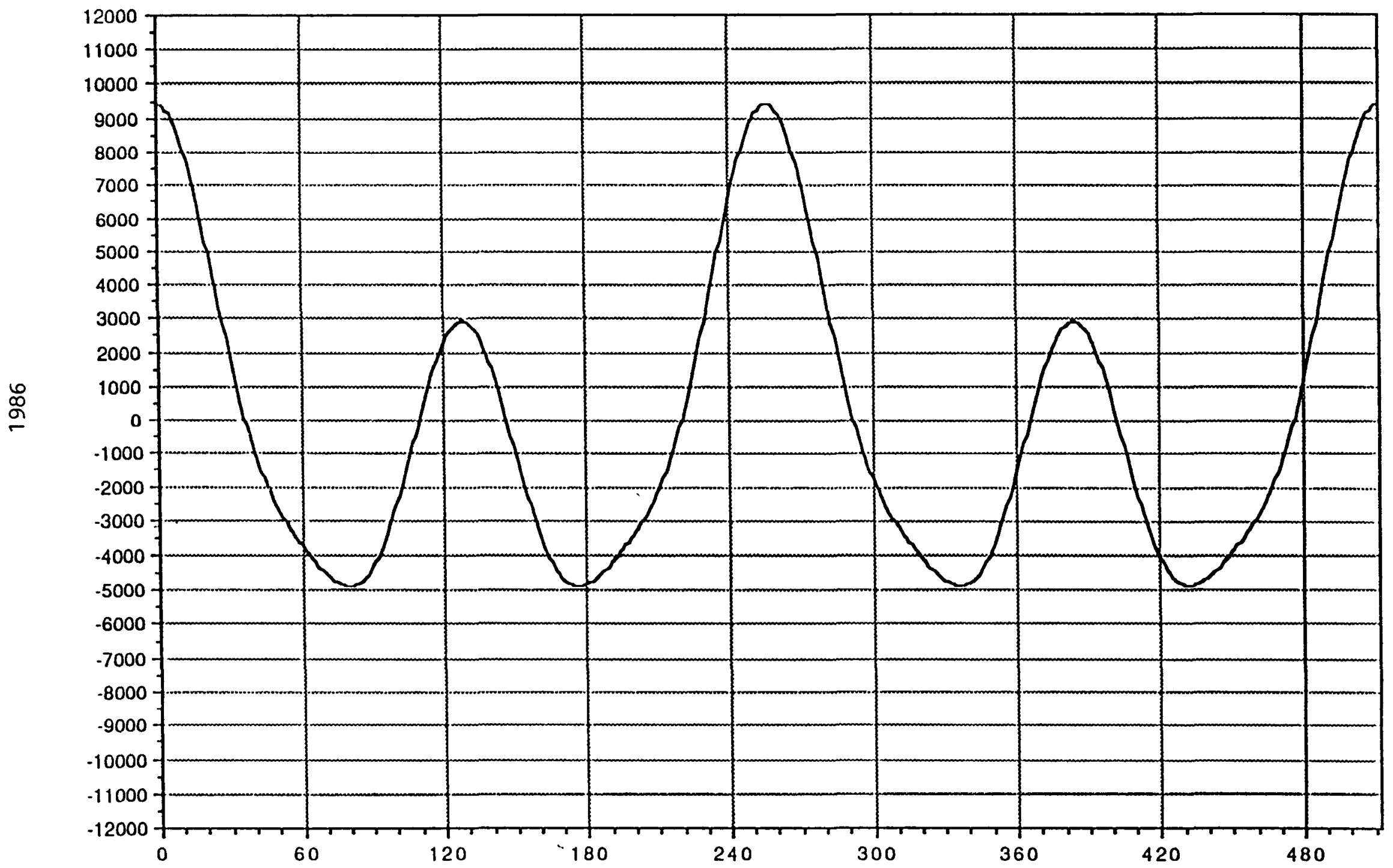




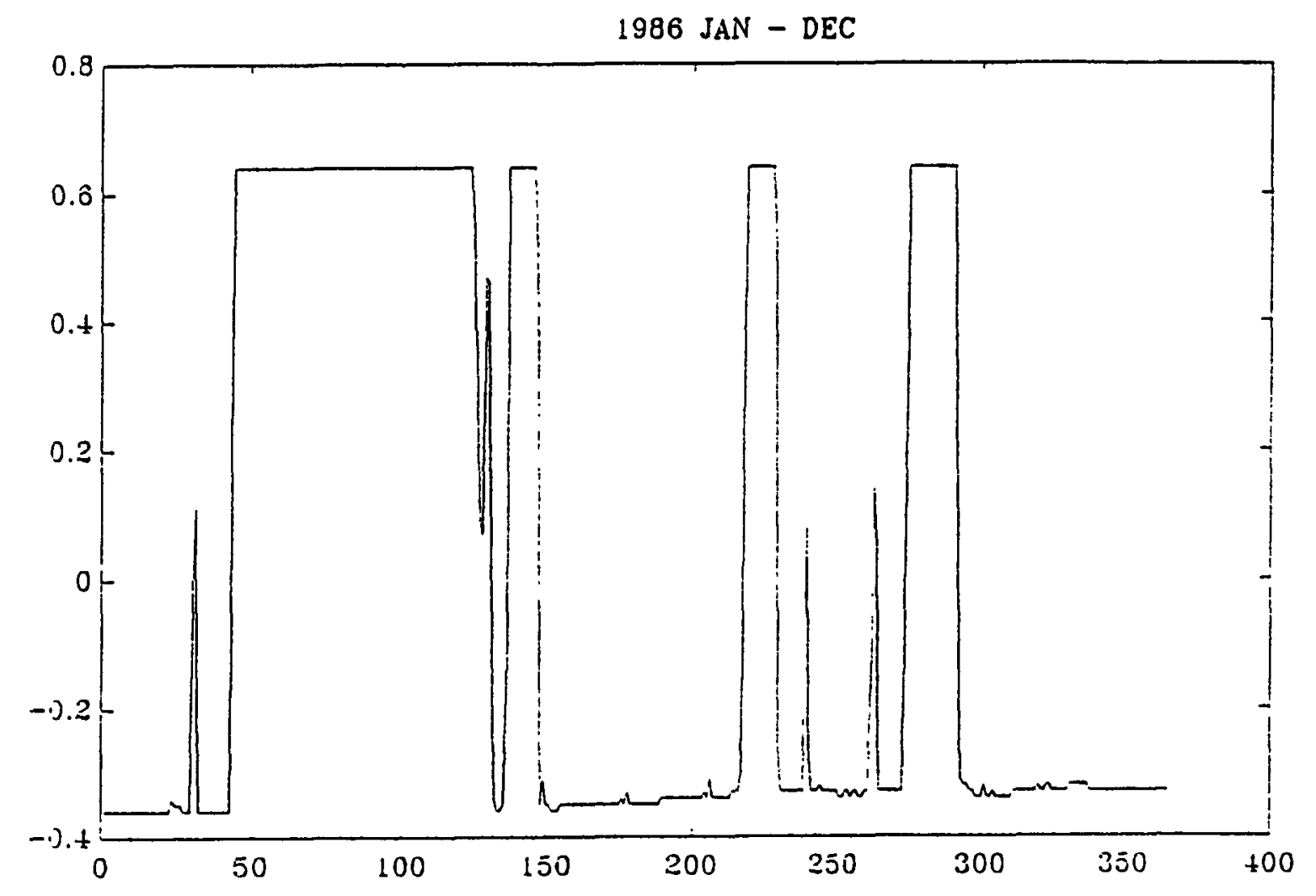

Att-1-82 


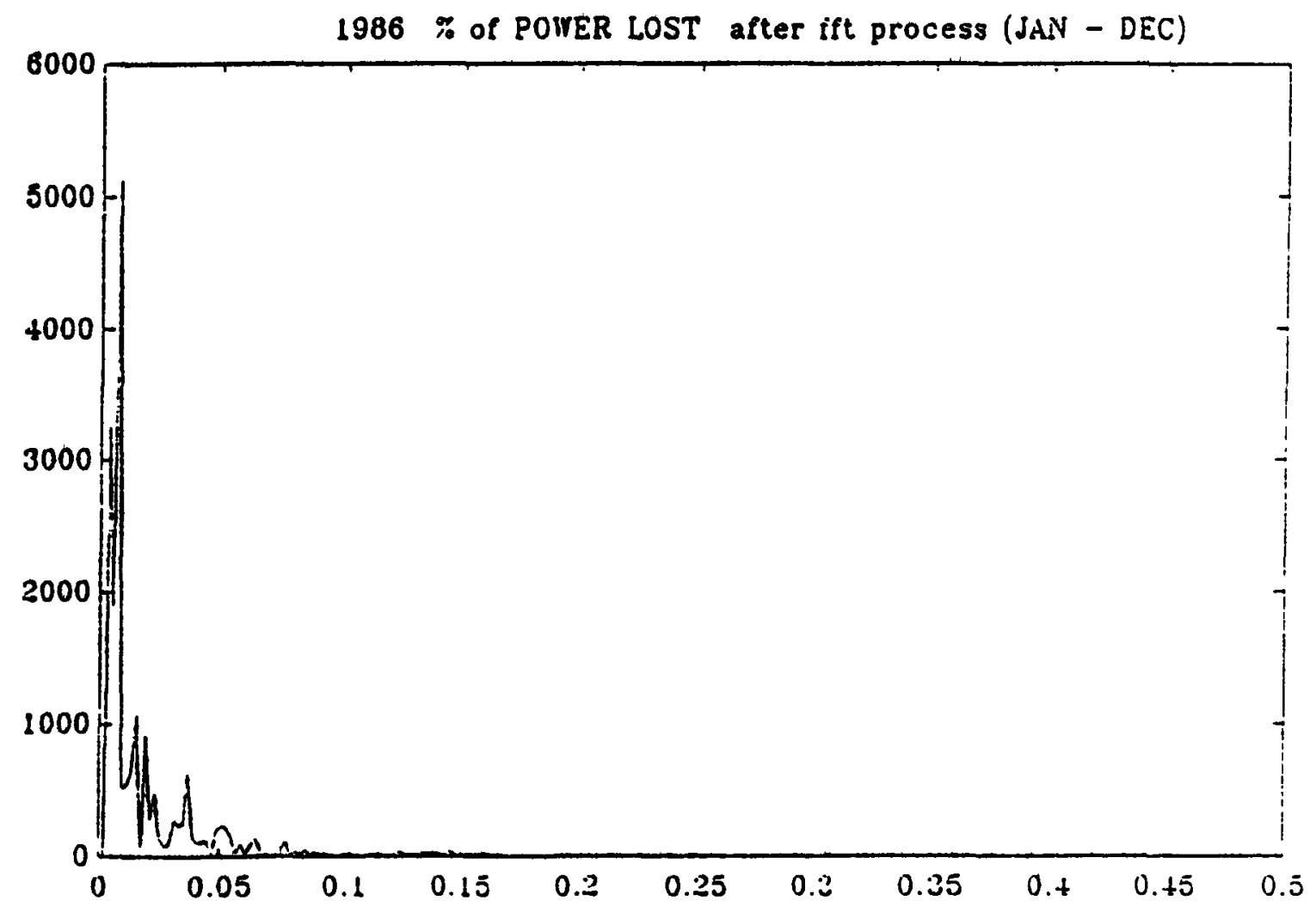




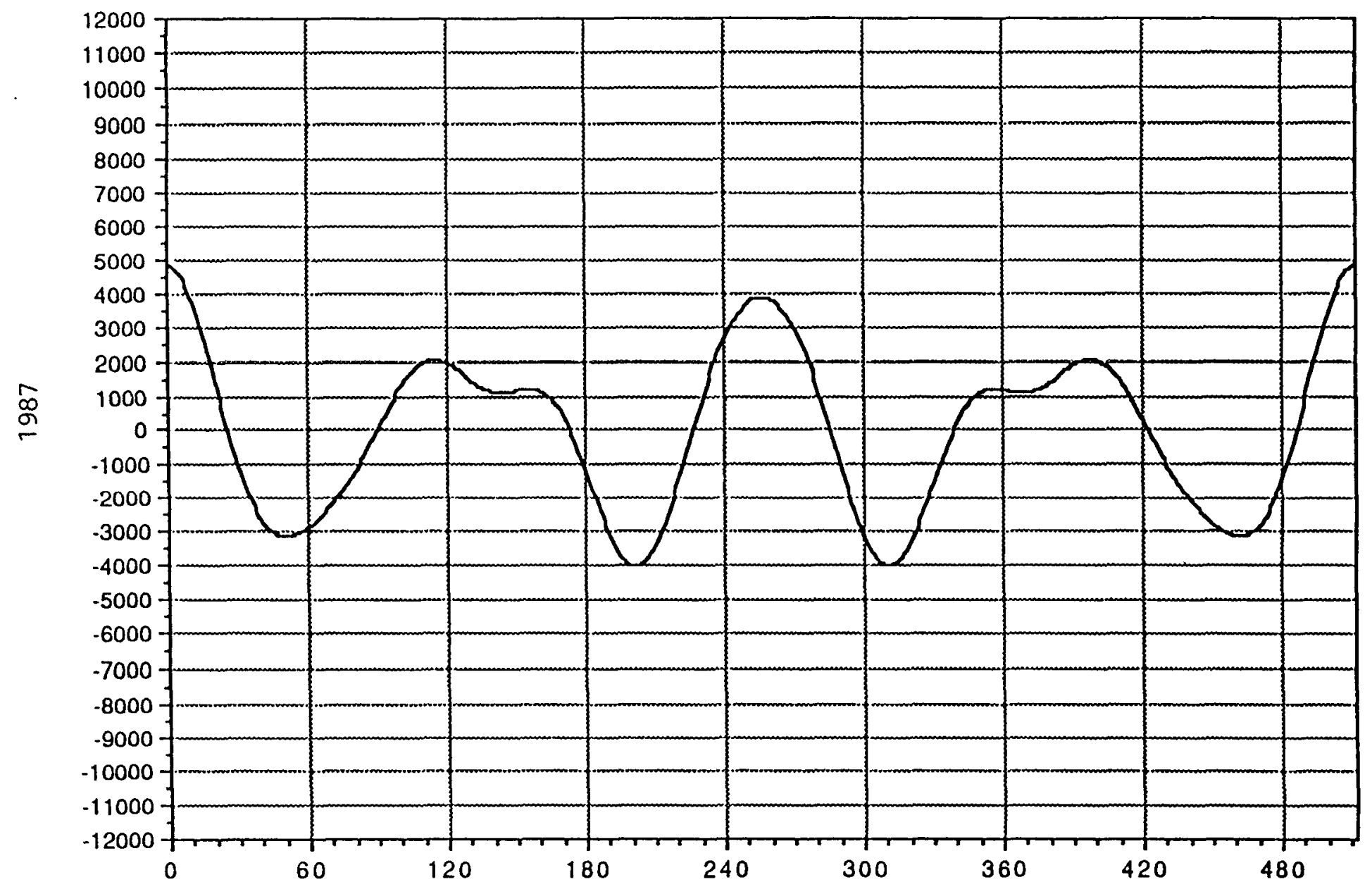




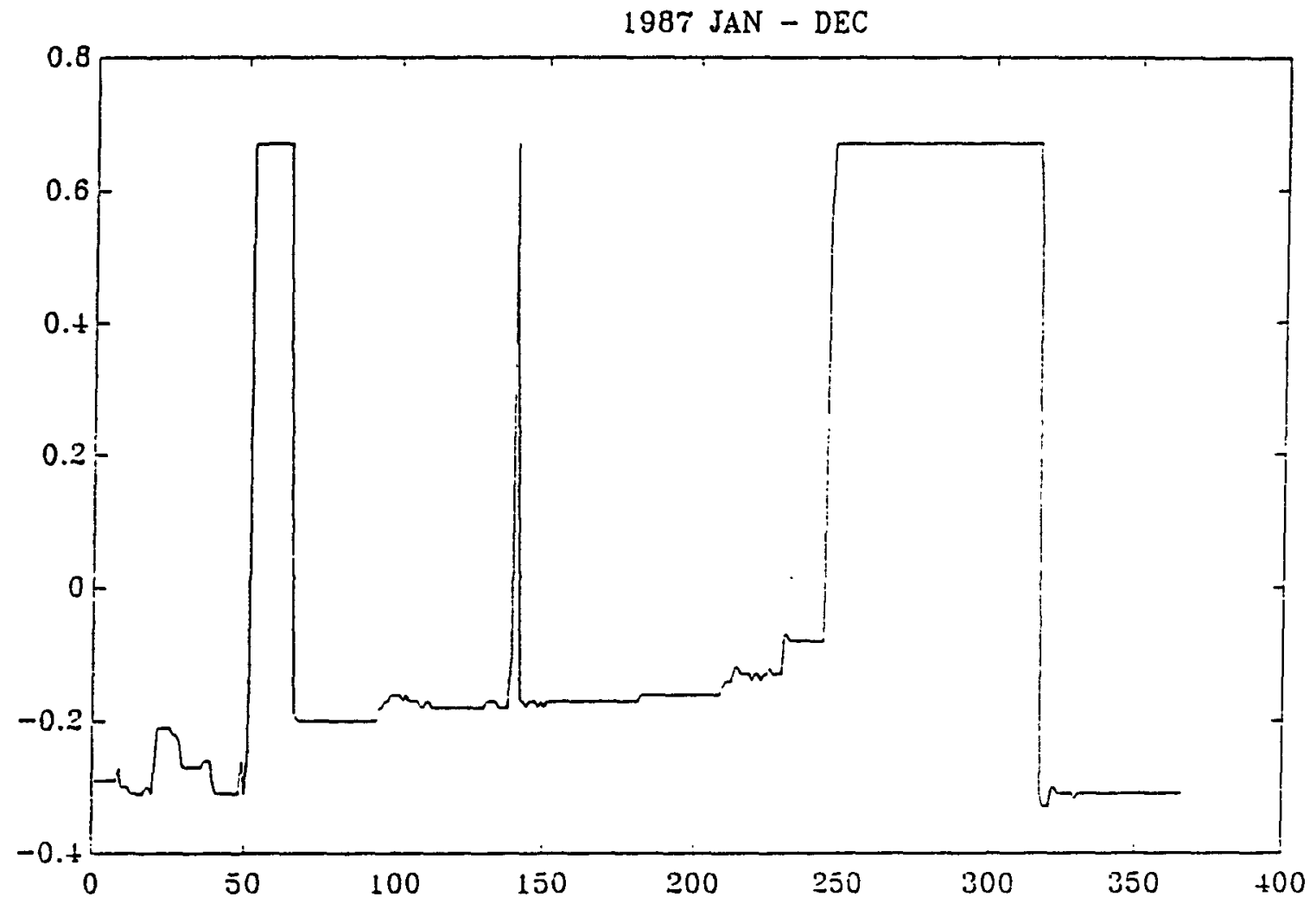




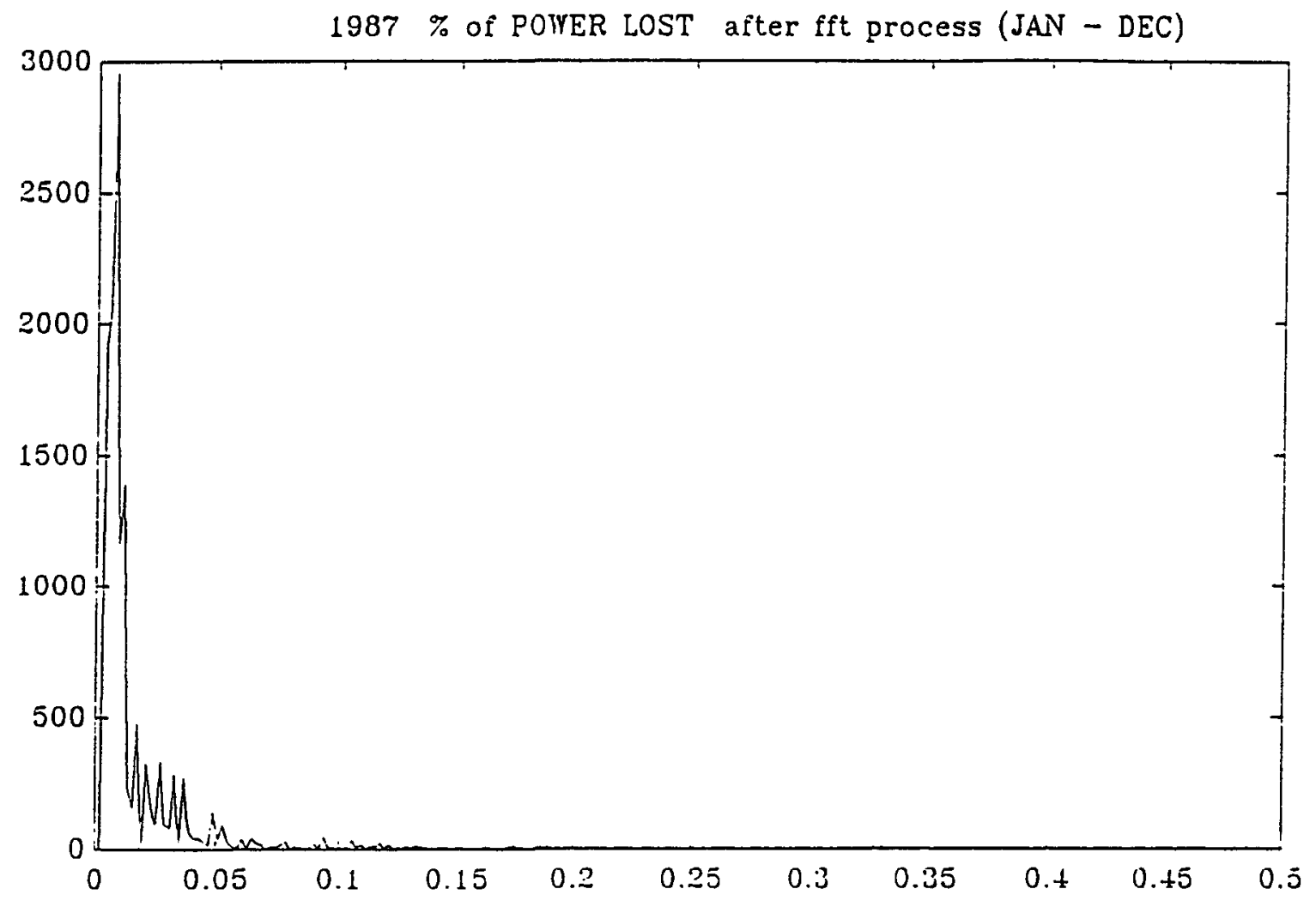

Att-1-86 


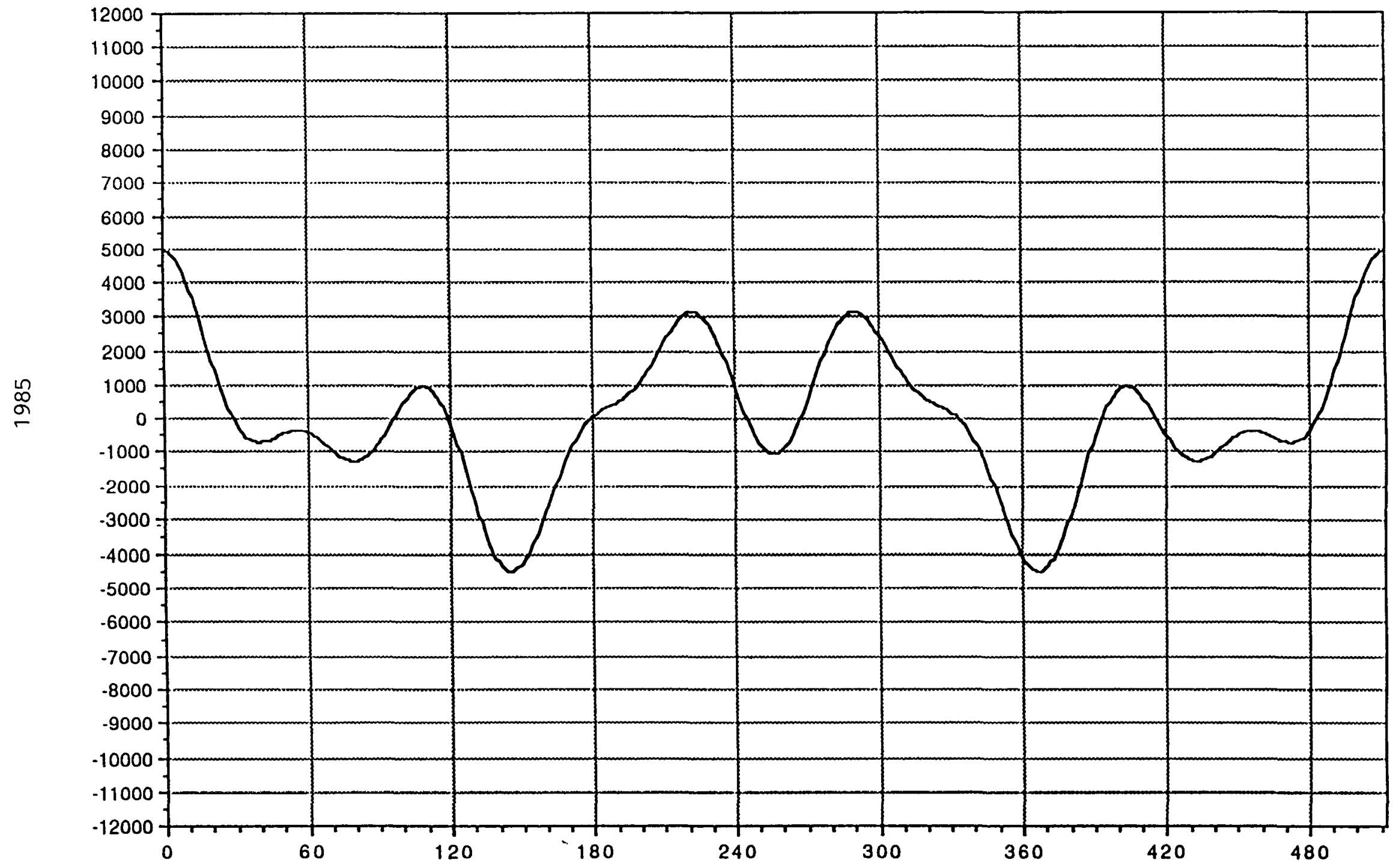




\section{Detailed Results for Plant Y}




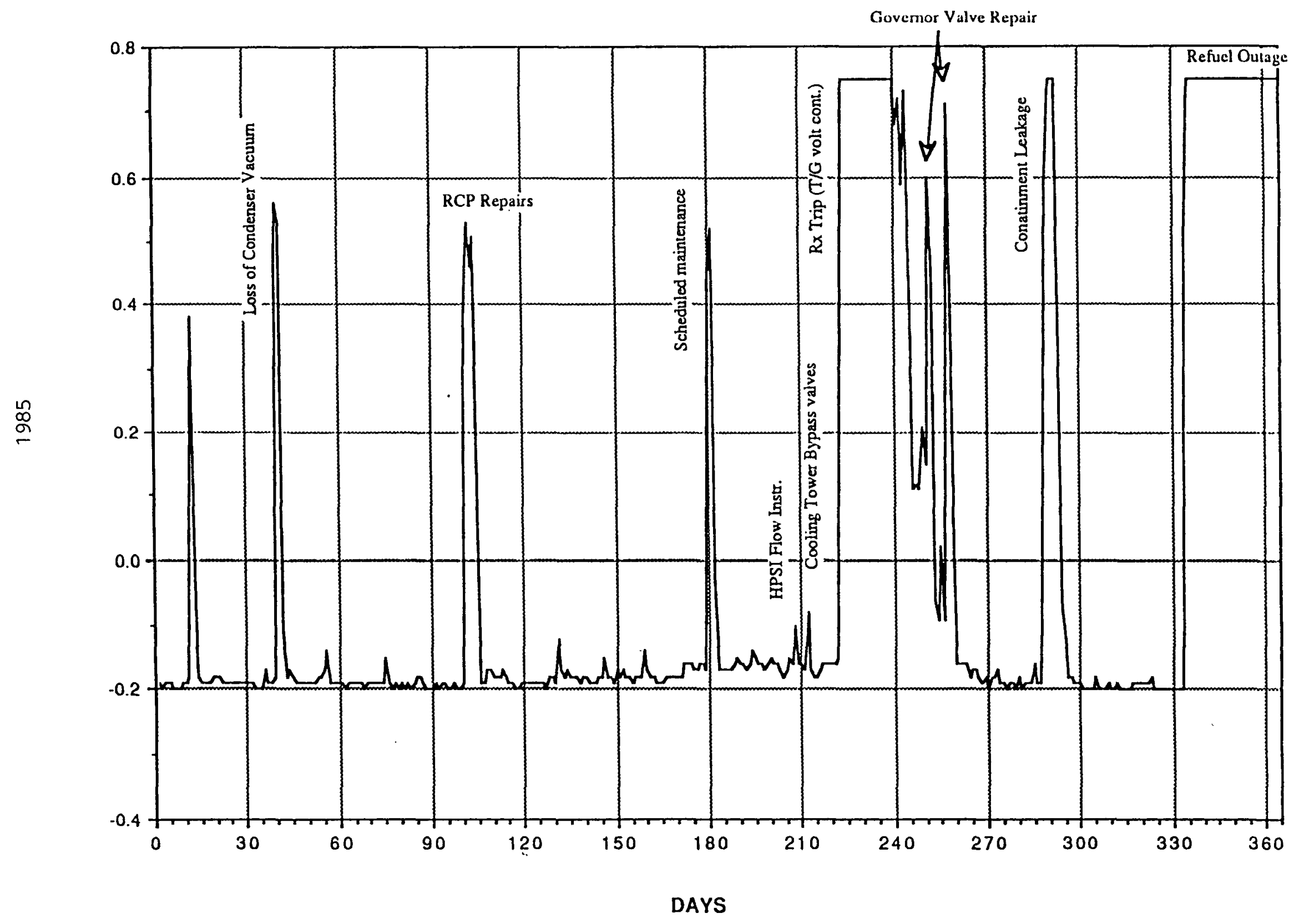

\begin{tabular}{l}
$\infty$ \\
1 \\
$\vdots$ \\
\multirow{1}{*}{}
\end{tabular} 


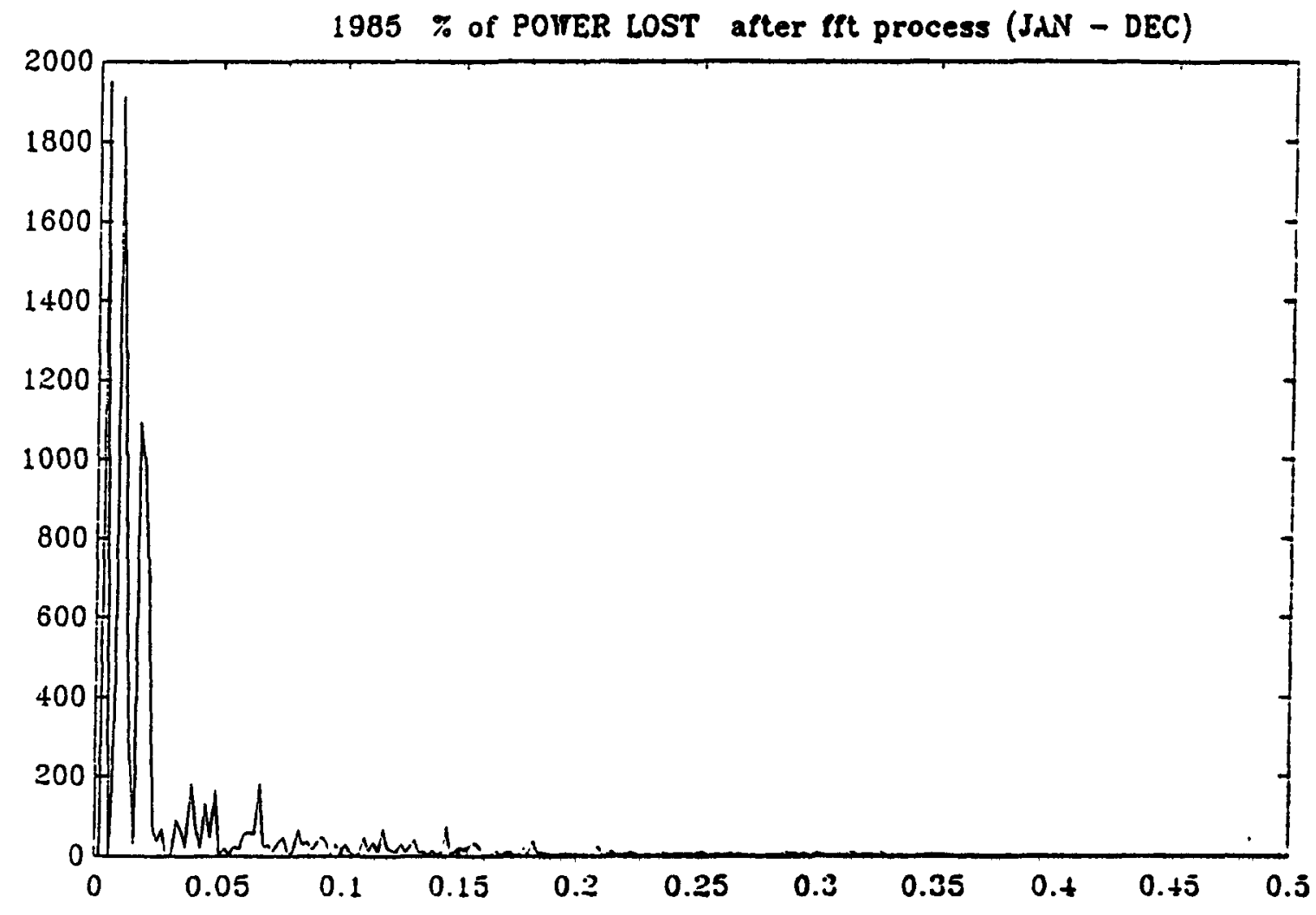




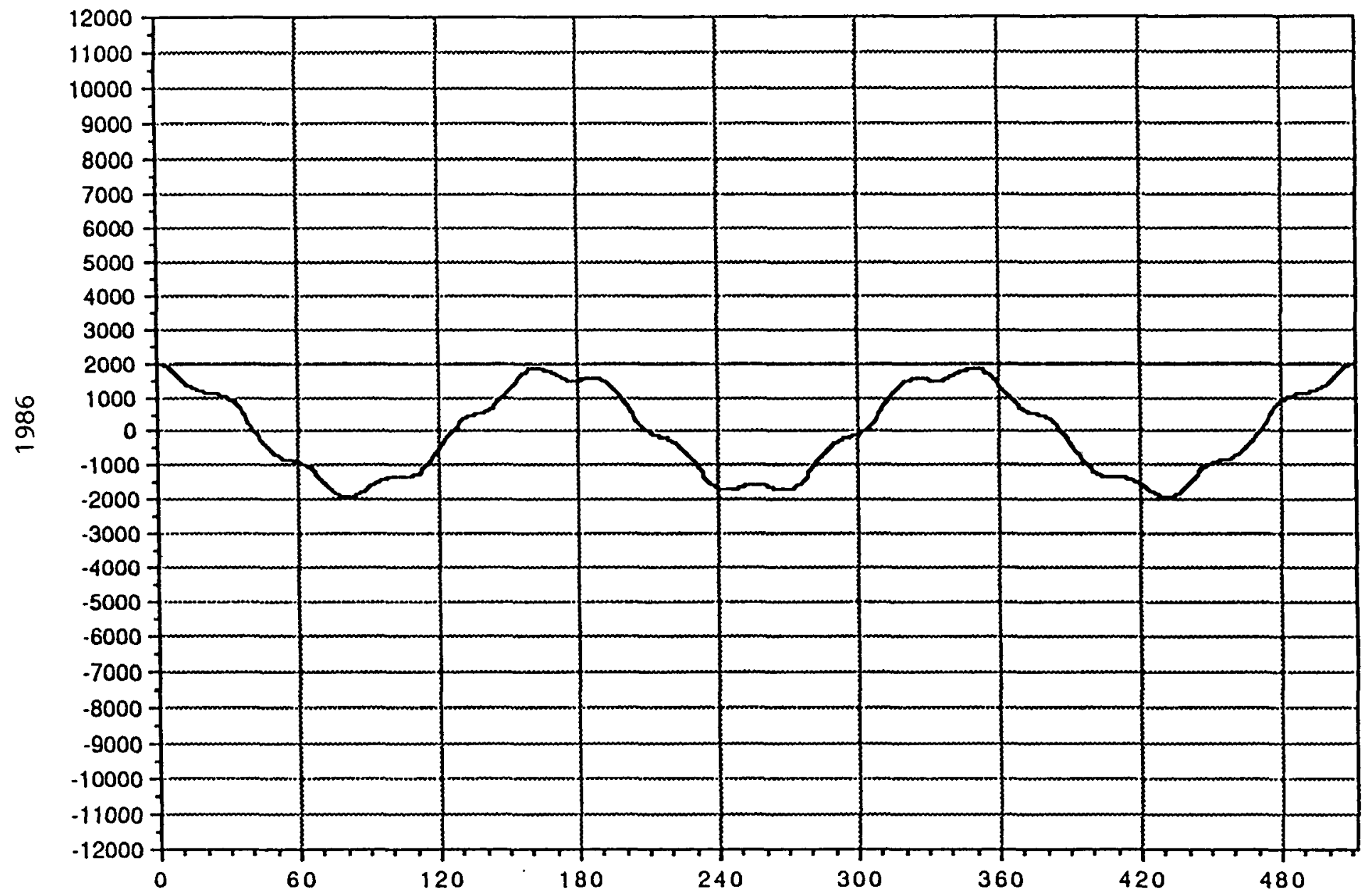




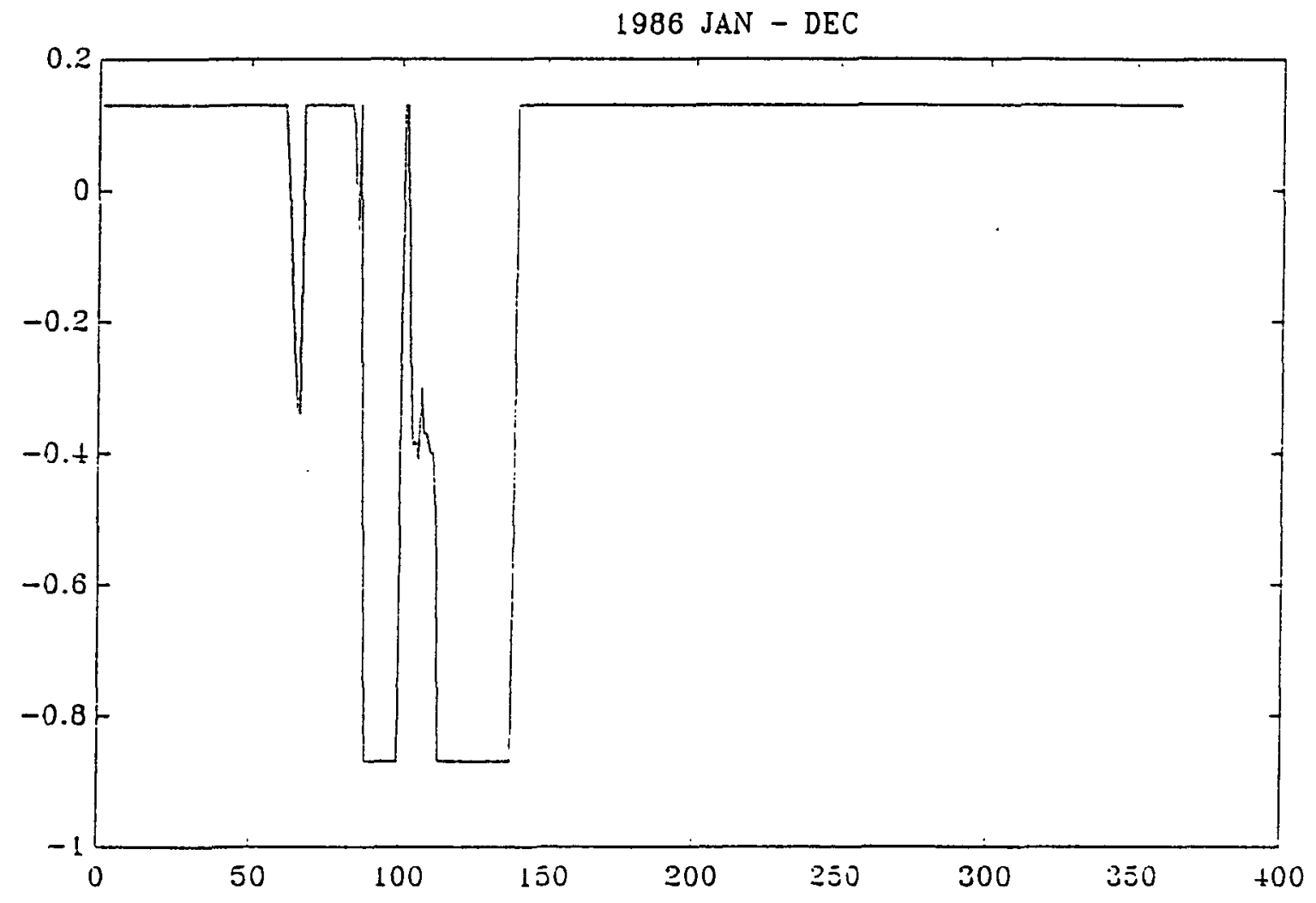

Att-1-92 


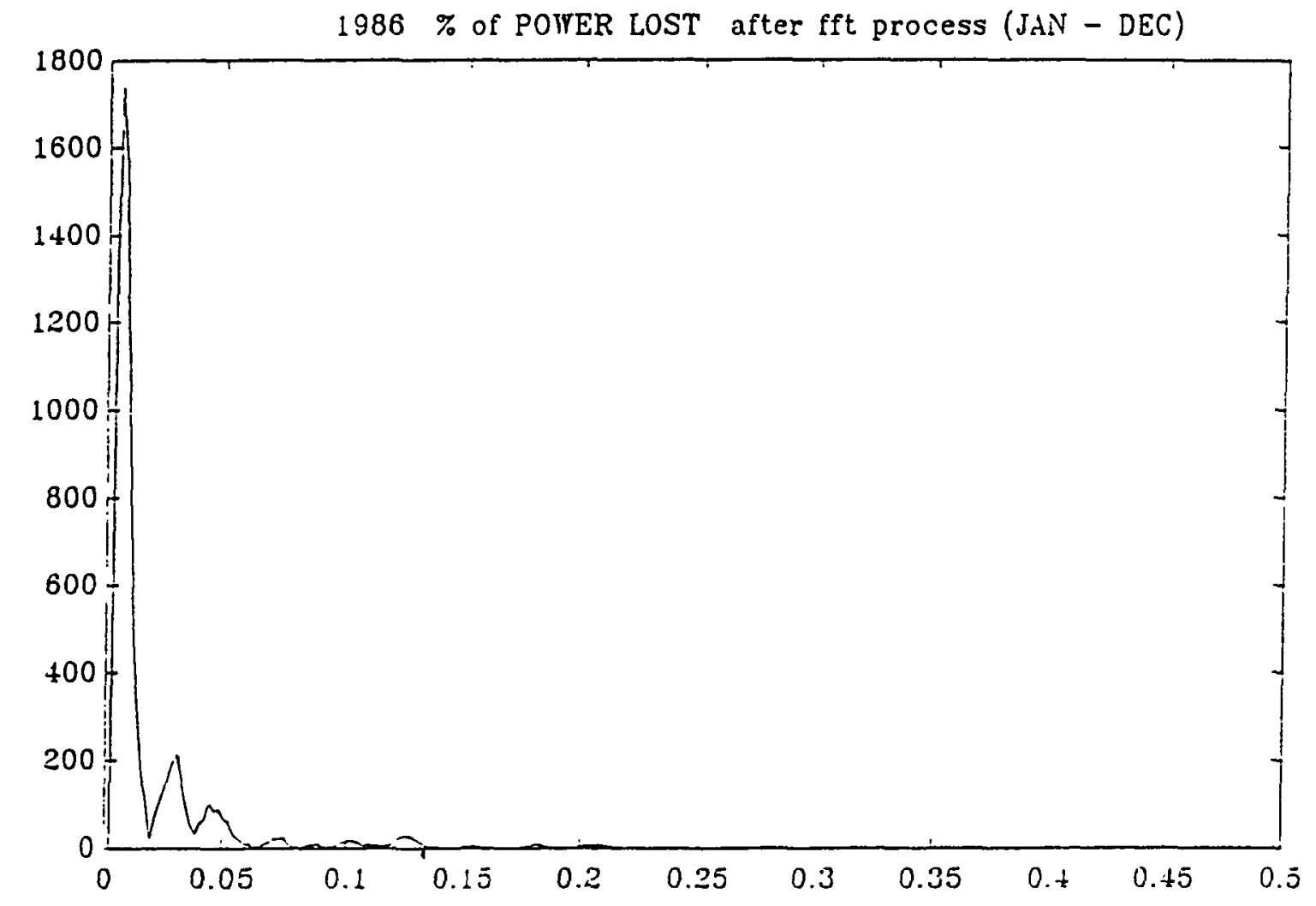

Att-1-93 







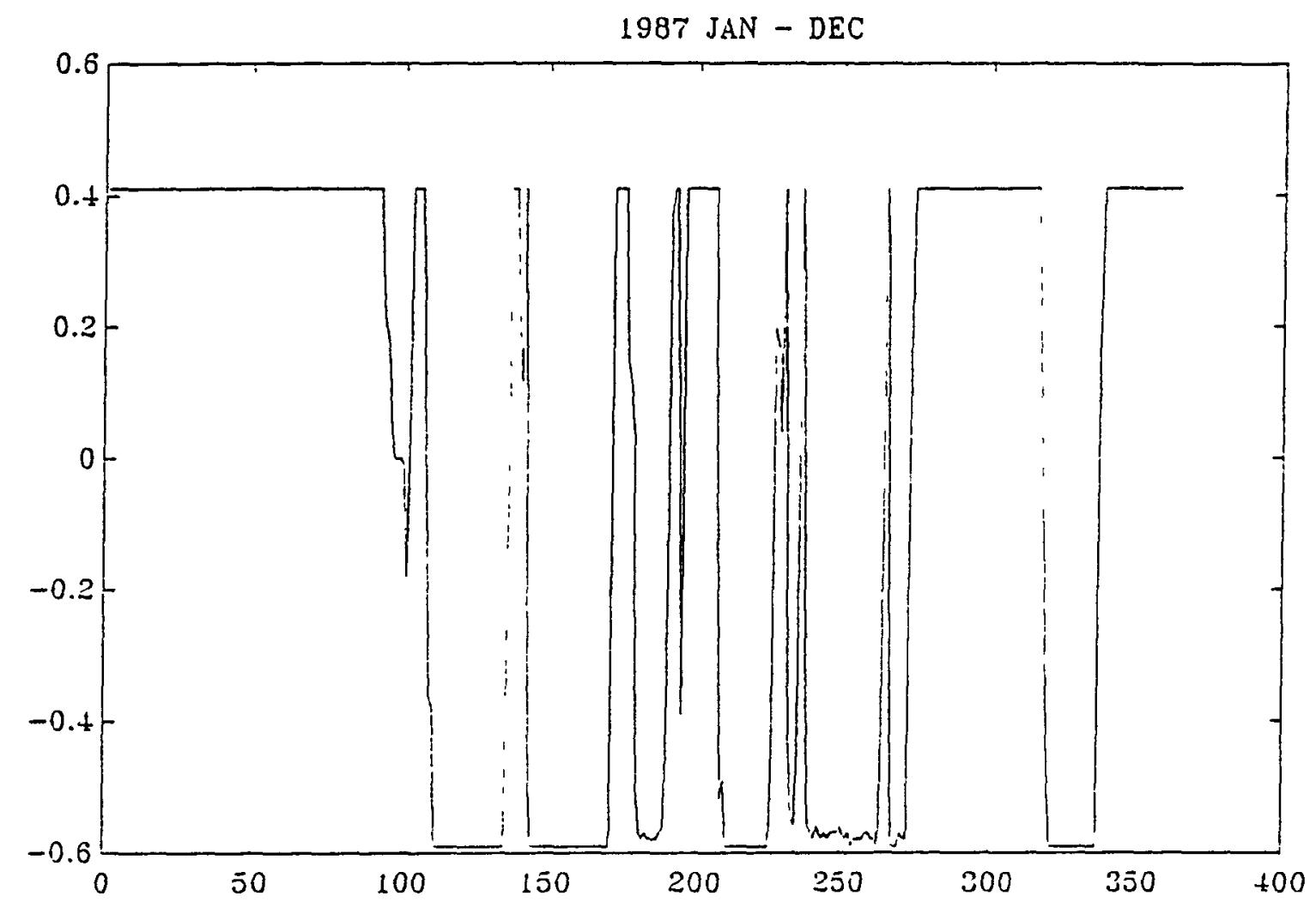




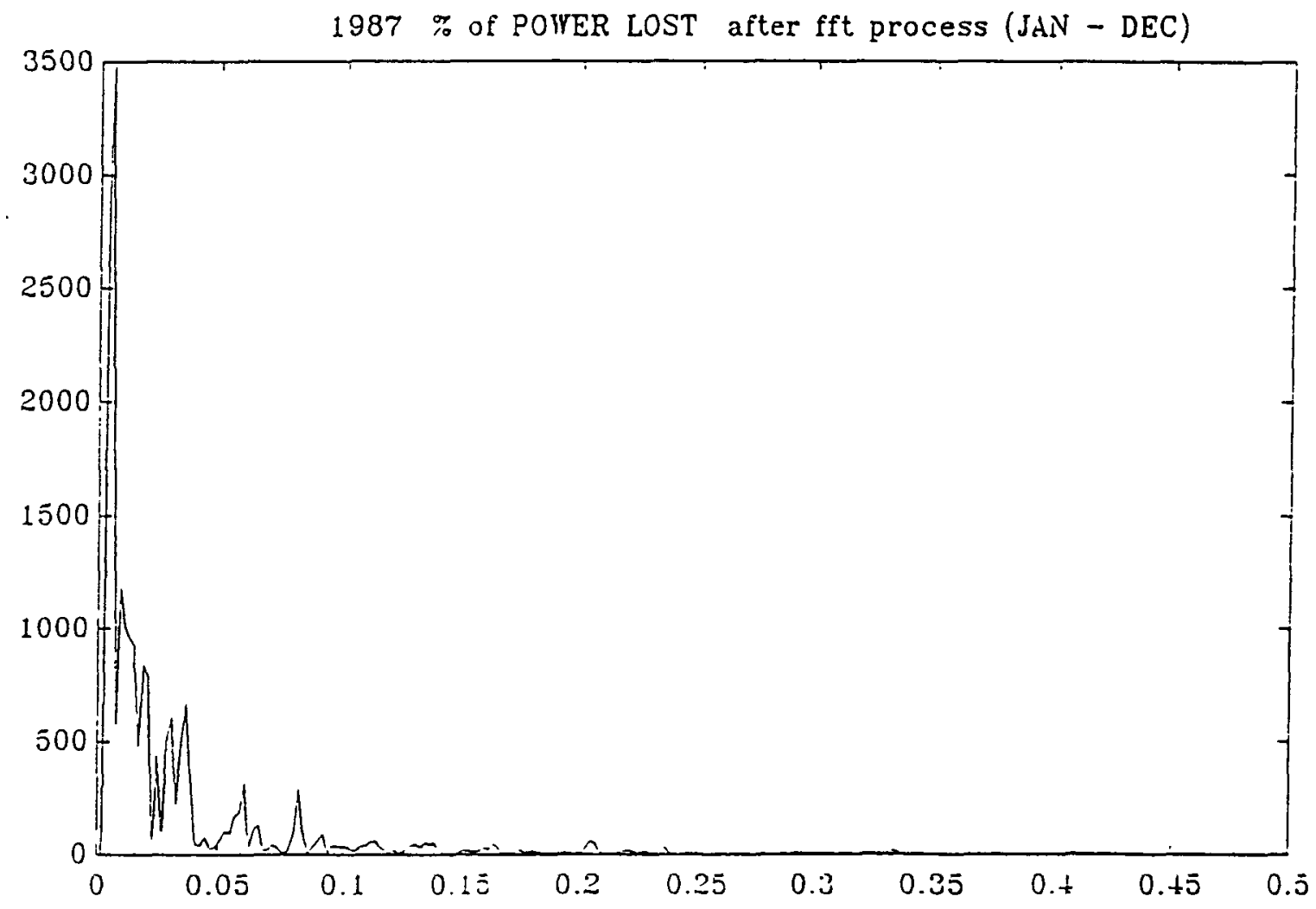


Detailed Results for Plant $Z$ 







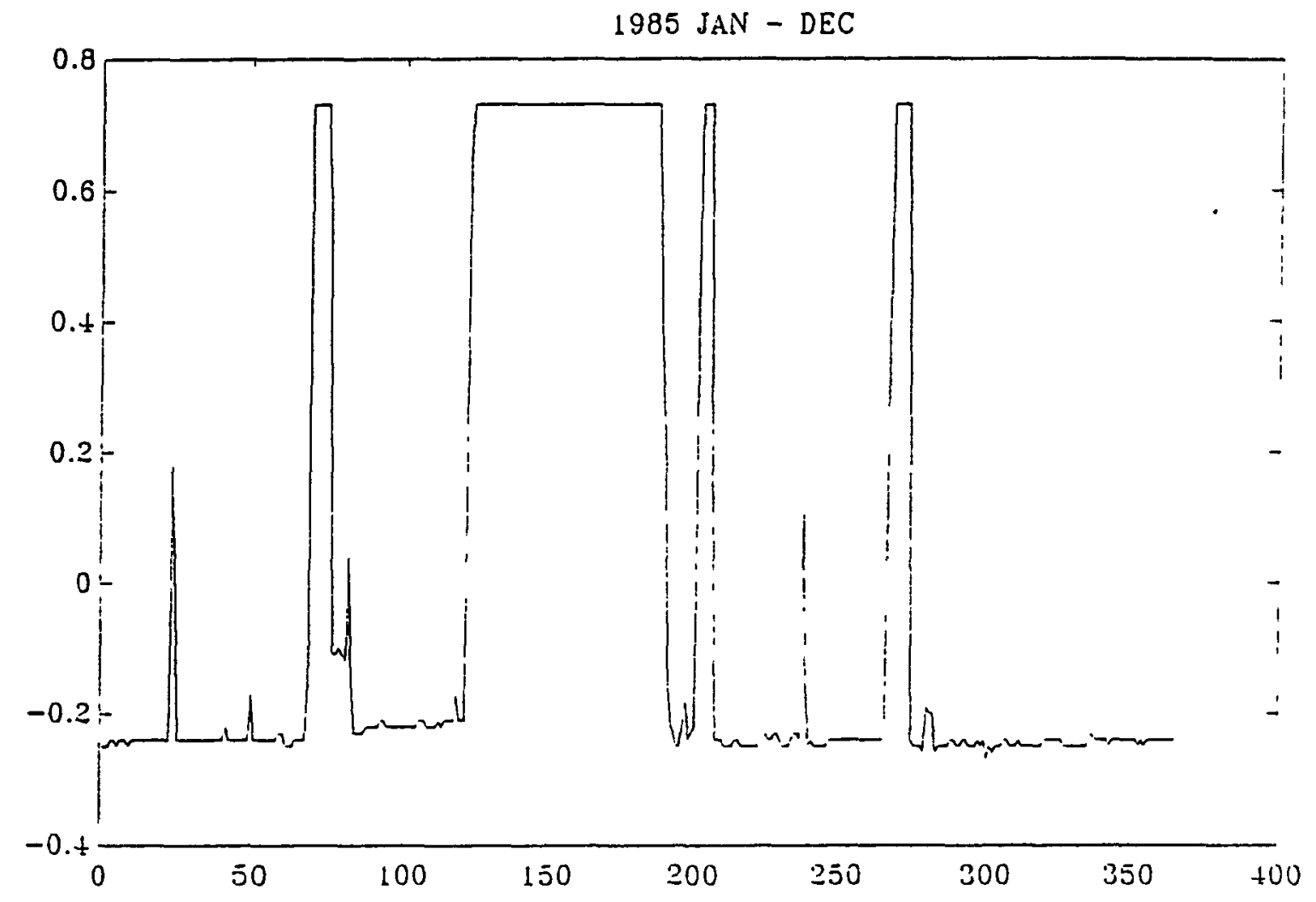




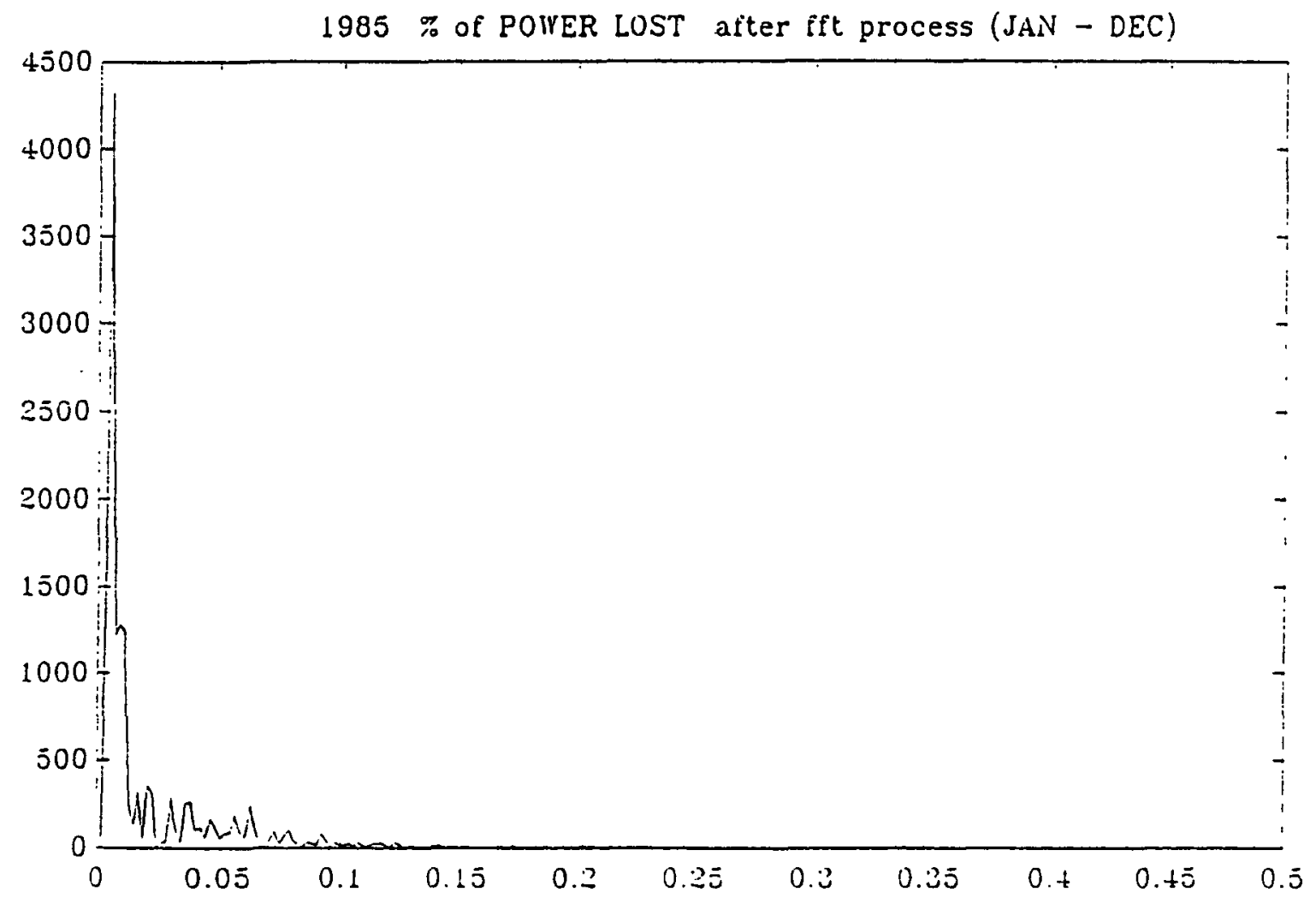




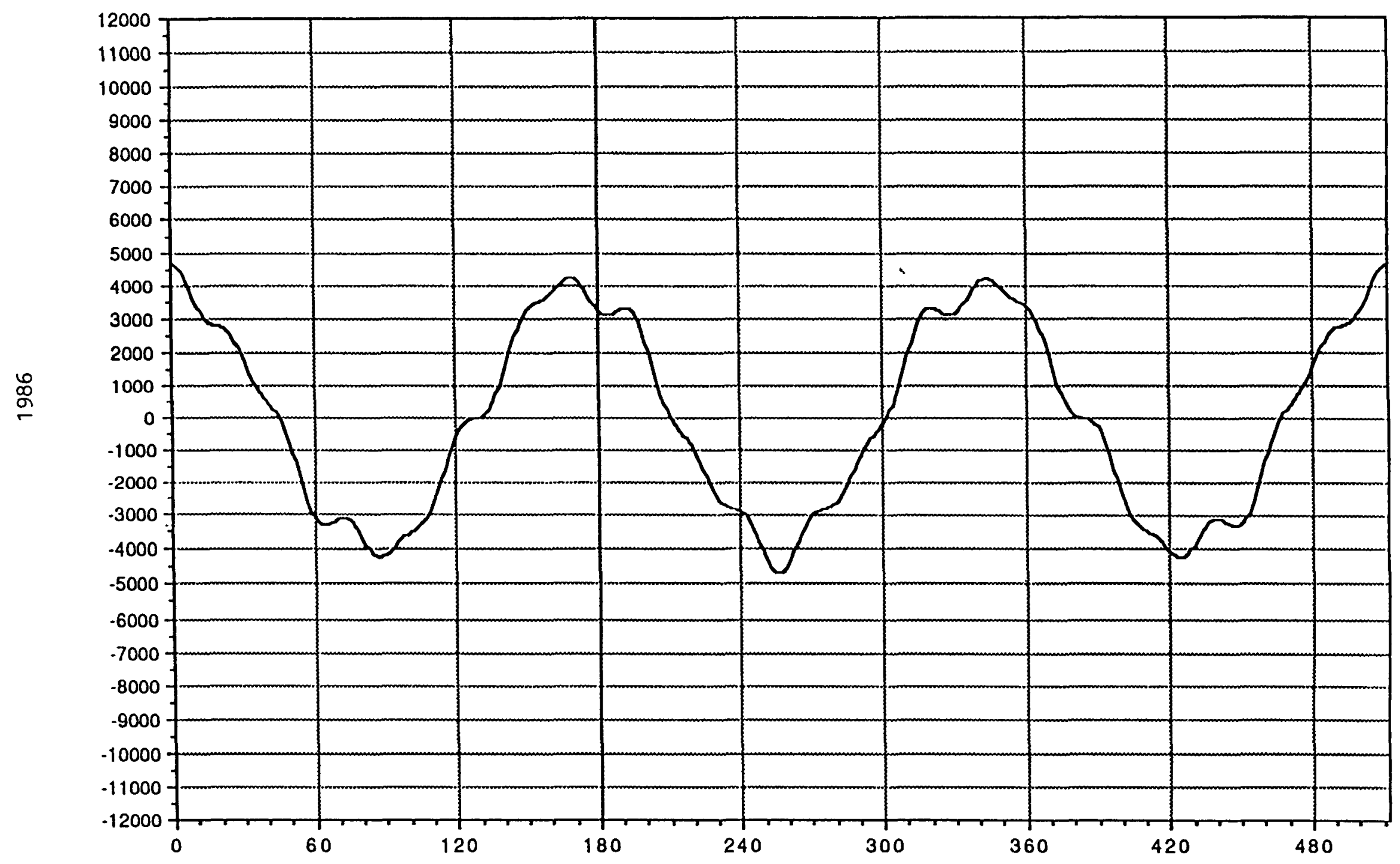




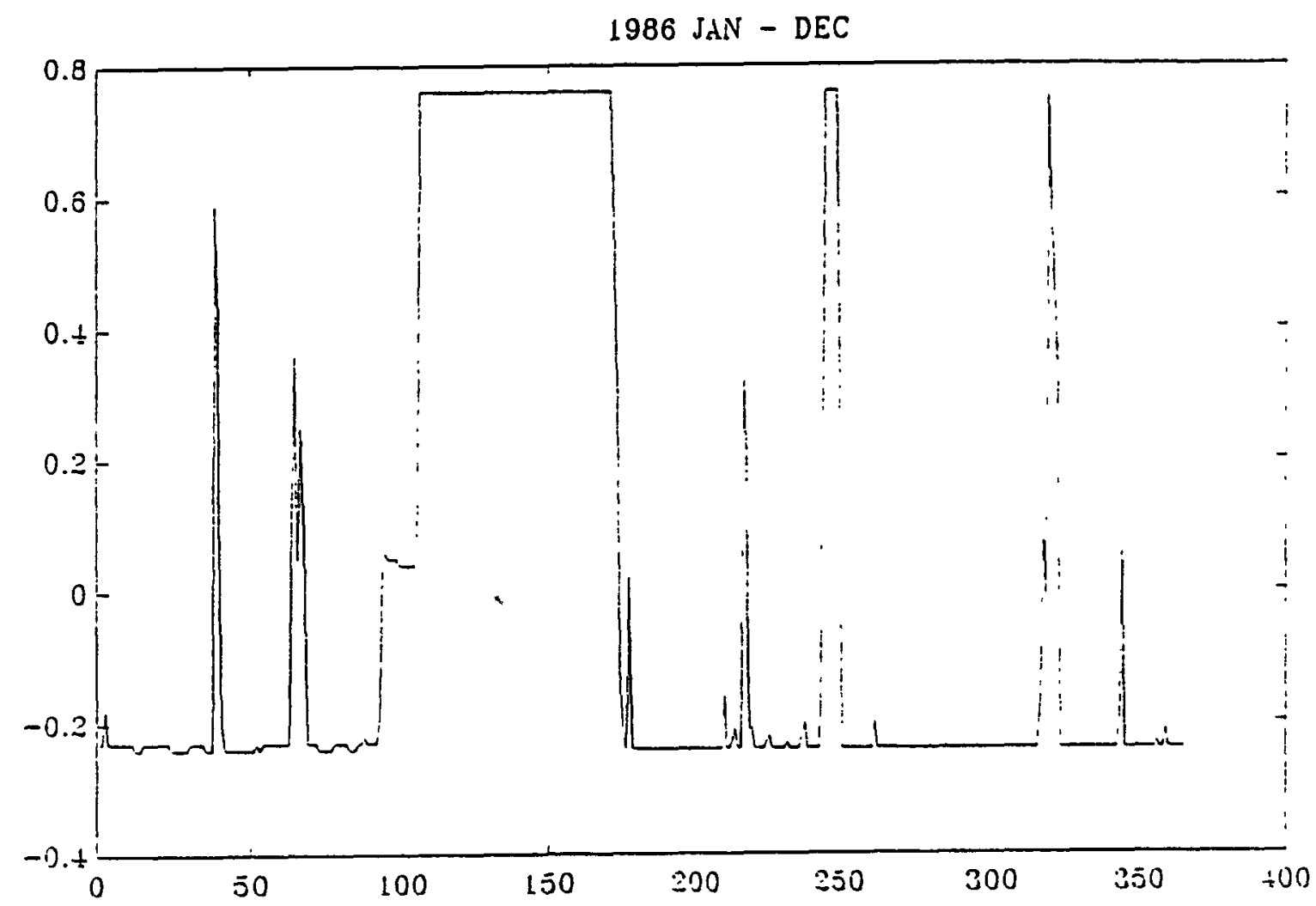




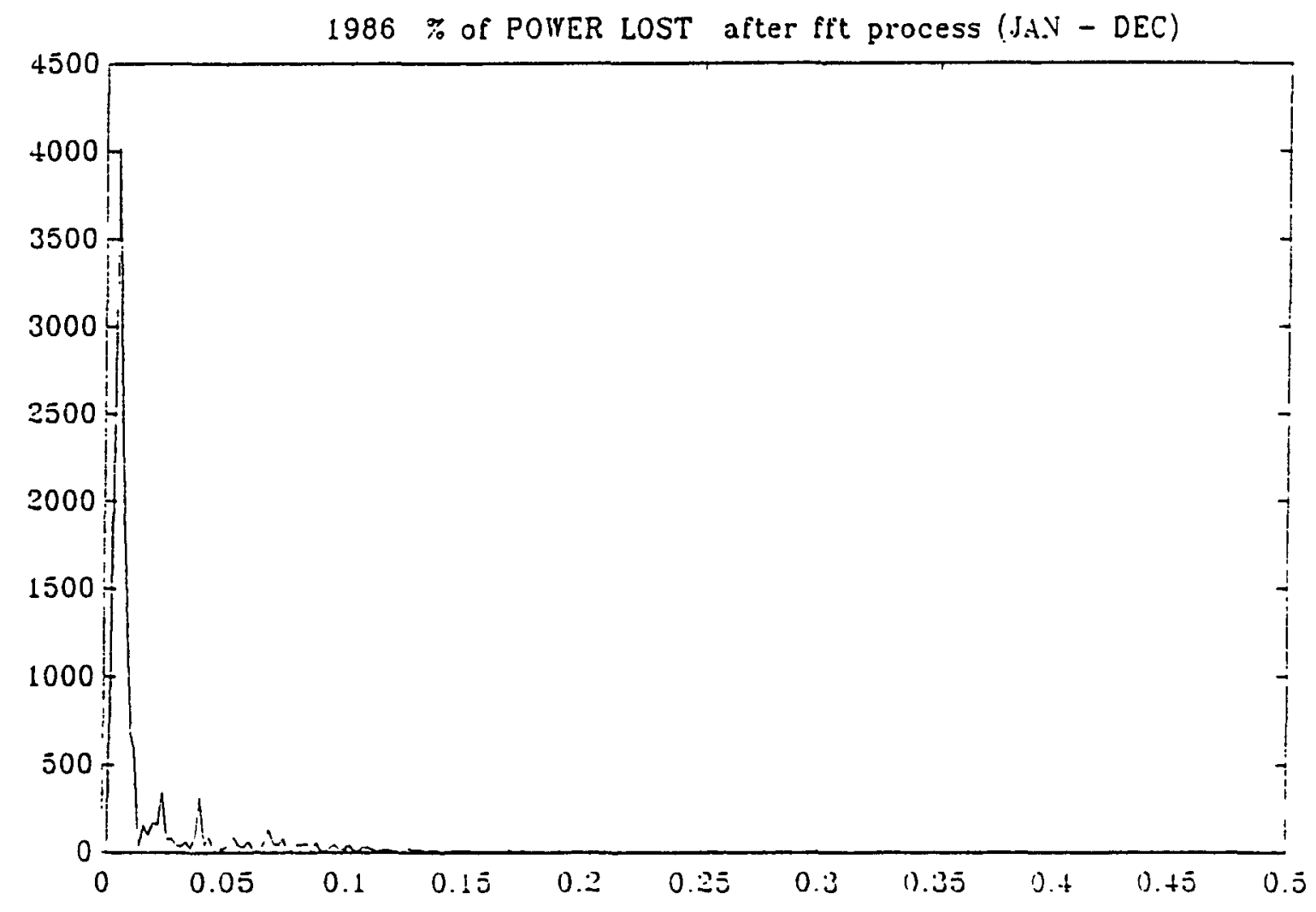

Att-1-103 


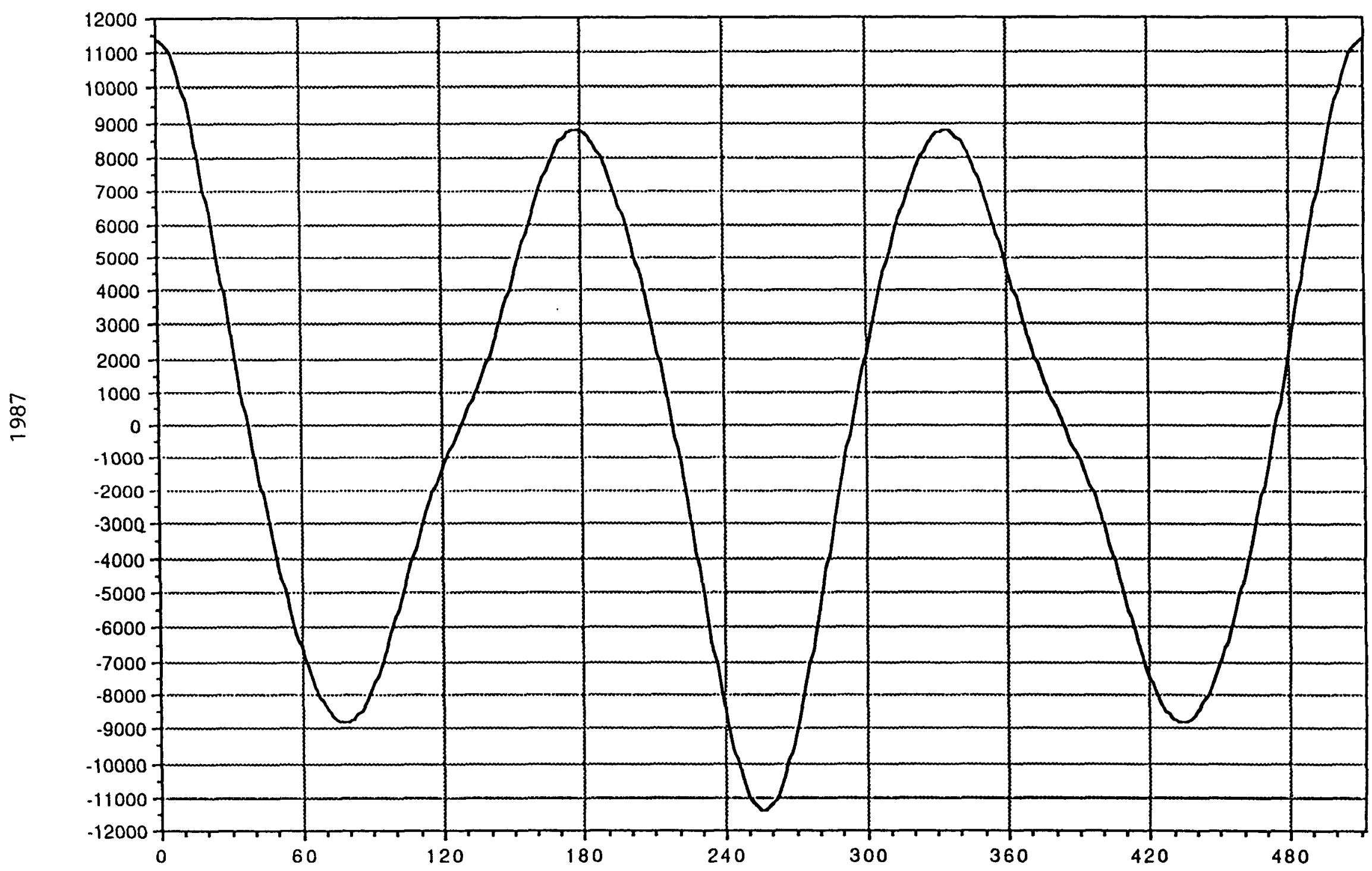




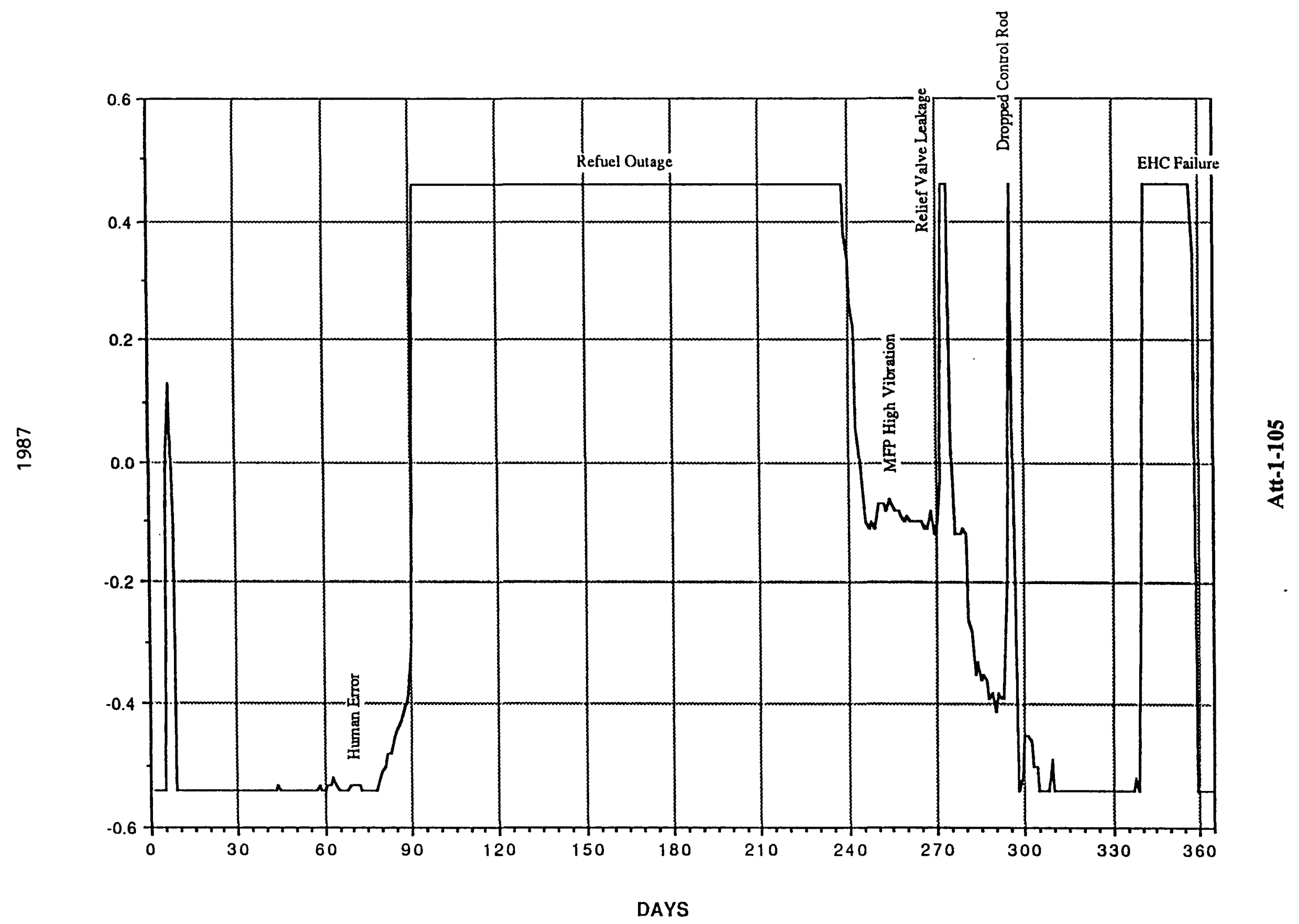




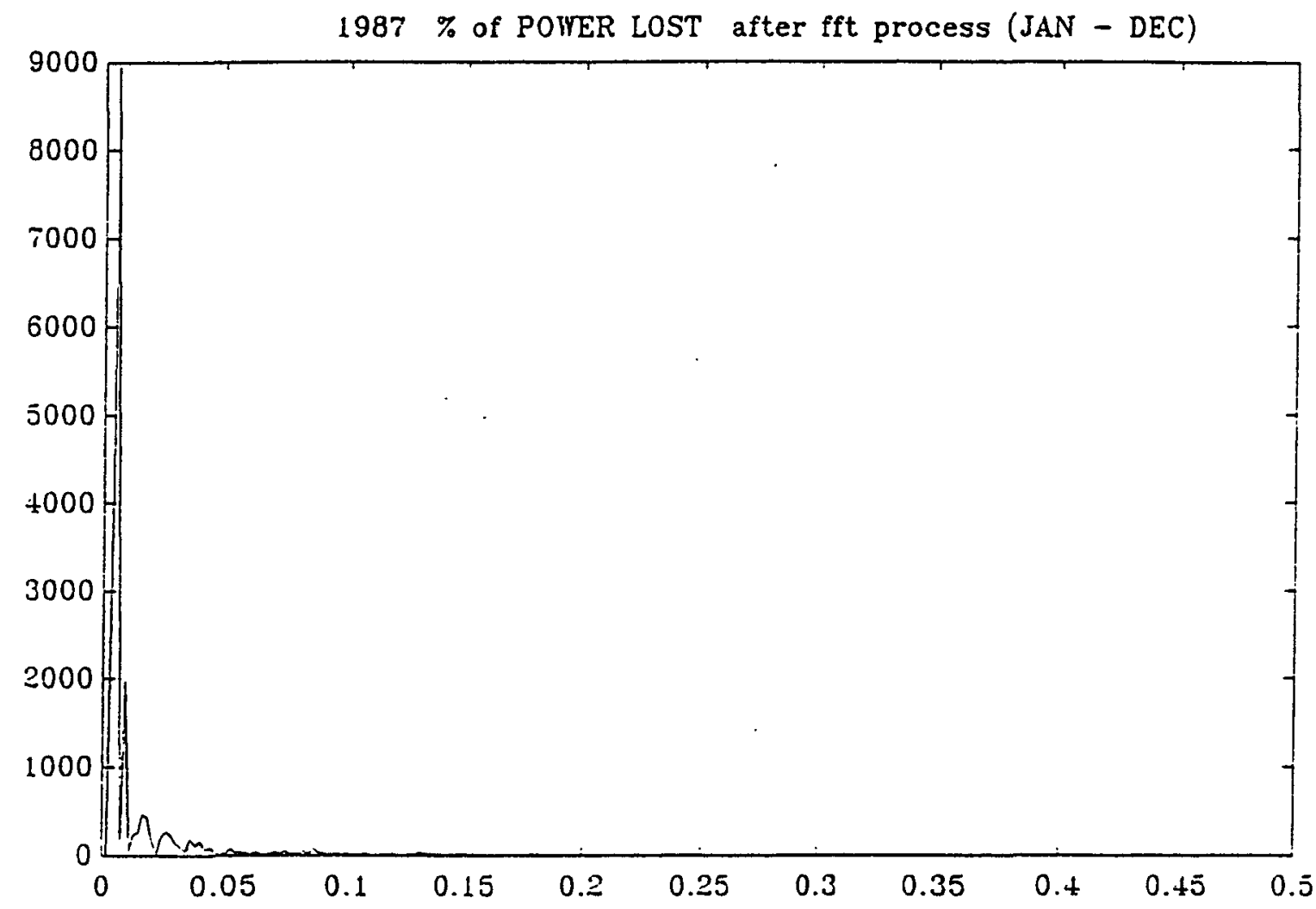

